JOURNAL OF

SYMPLECTIC GEOMETRY

Volume 3, Number 1, 55-159, 2005

\title{
GROMOV-WITTEN INVARIANTS OF SYMPLECTIC QUOTIENTS AND ADIABATIC LIMITS
}

\author{
Ana Rita Pires Gaio and Dietmar A. Salamon
}

We study pseudoholomorphic curves in symplectic quotients as adiabatic limits of solutions to the symplectic vortex equations. Our main theorem asserts that the genus zero invariants of Hamiltonian group actions defined by these equations are related to the genus zero Gromov-Witten invariants of the symplectic quotient (in the monotone case) via a natural ring homomorphism from the equivariant cohomology of the ambient space to the quantum cohomology of the quotient.

Contents

1. Introduction $\quad 56$

2. Pseudoholomorphic curves 65

3. Adiabatic limits $\quad 69$

4. Linear estimates $\quad 71$

5. Quadratic estimates 85

6. Proof of Theorem $3.1 \quad 88$

7. Relative Coulomb gauge 94

8. Proof of Theorem $3.2 \quad 106$

9. A priori estimates 116

10. Proof of Theorem $3.3 \quad 122$

$\begin{array}{ll}\text { 11. Vortices } & 129\end{array}$

12. Proof of Theorem $1.1 \quad 136$

Appendix A. The graph construction 149

Appendix B. Cauchy-Riemann operators 151

Appendix C. Invariant metrics $\quad 155$

$\begin{array}{ll}\text { References } & 158\end{array}$ 


\section{Introduction}

The main theorem of this paper asserts that under certain hypotheses there is a ring homomorphism from the equivariant cohomology of a symplectic manifold $M$ with a Hamiltonian G-action to the quantum cohomology of the symplectic quotient $\bar{M}$ such that the following diagram commutes

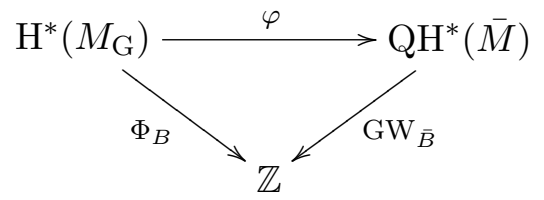

Here $\mathrm{GW}_{\bar{B}}$ denotes the genus zero Gromov-Witten invariants of $\bar{M}$ with fixed marked points associated to a homology class $\bar{B} \in H_{2}(\bar{M} ; \mathbb{Z})$ and $\Phi_{B}$ denotes the genus zero invariants of Hamiltonian group actions associated to the equivariant homology class $B=\kappa(\bar{B}) \in H_{2}\left(M_{\mathrm{G}} ; \mathbb{Z}\right)$. The latter invariant was introduced in $[\mathbf{2}, \mathbf{3}, \mathbf{1 3}, \mathbf{1 4}]$. The homomorphism $\varphi$ is defined indirectly as a consequence of a comparison theorem for the two invariants. A more direct definition in terms of vortices over the complex plane with values in $M$ will be given elsewhere. The proof of the comparison theorem is based on an adiabatic limit analysis which relates the solutions of the symplectic vortex equations to pseudoholomorphic curves in the symplectic quotient. Our hypotheses are that the moment map is proper, that $M$ is convex at infinity, and that the quotient $\bar{M}$ is smooth; these hypotheses are needed to even state the result. We also assume that there are no holomorphic spheres in the ambient manifold (and hence $M$ is necessarily noncompact); Corollary 1.2 cannot be expected to be true without it. Finally, we assume that $\bar{M}$ is monotone; this hypothesis is of a technical nature and it might be possible to remove it. But this would require more analysis than is carried out in the present paper. Before stating the main results more precisely (Theorem 1.1 and Corollary 1.2), we review the invariants introduced in [2]. Invariants of Hamiltonian group actions. Let $(M, \omega)$ be a symplectic manifold (not necessarily compact) and $\mathrm{G}$ be a compact connected Lie group with Lie algebra $\mathfrak{g}$. We fix an invariant inner product $\langle\cdot, \cdot\rangle$ on $\mathfrak{g}$ and identify $\mathfrak{g}$ with its dual $\mathfrak{g}^{*}$. We assume that $\mathrm{G}$ acts on $M$ by Hamiltonian symplectomorphisms and that the action is generated by an equivariant moment map $\mu: M \rightarrow \mathfrak{g}$. This means that, for every $\eta \in \mathfrak{g}$, the vector field $X_{\eta} \in \operatorname{Vect}(M)$ that generates the action is determined by $\iota\left(X_{\eta}\right) \omega=d\langle\mu, \eta\rangle$. Let $\pi: P \rightarrow \Sigma$ be a principal G-bundle over a compact oriented Riemann surface $\left(\Sigma, j_{\Sigma}, \mathrm{dvol}_{\Sigma}\right)$. We fix a smooth family $\Sigma \rightarrow \mathcal{J}_{\mathrm{G}}(M, \omega): z \mapsto J_{z}$ of G-invariant and $\omega$-compatible almost complex structures on $M$. This determines a family of metrics $\langle\cdot, \cdot\rangle_{z}:=\omega\left(\cdot, J_{z} \cdot\right)$. The invariants are derived from the equations

$$
\bar{\partial}_{J, A}(u)=0, \quad * F_{A}+\mu(u)=0,
$$


for a pair $(u, A)$, where $u: P \rightarrow M$ is a G-equivariant map and $A$ a connection on $P$. Here $F_{A}$ is the curvature of $A$ and $\bar{\partial}_{J, A}$ the nonlinear CauchyRiemann operator twisted by $A$ (see $[\mathbf{2}, \mathbf{3}]$ for a detailed explanation). Both terms in the second identity in equations (1.1) are sections of the Lie algebra bundle $\mathfrak{g}_{P}:=P \times_{\text {Ad }} \mathfrak{g}$. In contrast to the theory of pseudoholomorphic curves, equations (1.1) involve the volume form $\operatorname{dvol}_{\Sigma}$ (via the Hodge *-operator in the second equation) and not just the complex structure $j_{\Sigma}$. Equations (1.1) are invariant under the action of the gauge group $\mathcal{G}=\mathcal{G}(P)$ (of equivariant maps from $P$ to $\mathrm{G}$ ) by

$$
g^{*}(u, A)=\left(g^{-1} u, g^{-1} d g+g^{-1} A g\right) .
$$

From a geometric point of view, the solutions of equations (1.1) correspond to the absolute minima of the energy functional

$$
E(u, A)=\frac{1}{2} \int_{\Sigma}\left(\left|d_{A} u\right|^{2}+\left|F_{A}\right|^{2}+|\mu(u)|^{2}\right) \operatorname{dvol}_{\Sigma}
$$

in a given homotopy class. The solutions of equations (1.1) have energy

$$
E(u, A)=\int_{\Sigma}\left(u^{*} \omega-d\langle\mu(u), A\rangle\right)=:\langle[\omega-\mu],[u]\rangle,
$$

and this number is an invariant of the equivariant homology class represented by the map $u$. We impose the following hypothesis throughout this paper.

(H1): The moment map $\mu$ is proper, zero is a regular value of $\mu$, and $\mathrm{G}$ acts freely on $\mu^{-1}(0)$.

Under this hypothesis the quotient

$$
\bar{M}:=M / / \mathrm{G}:=\mu^{-1}(0) / \mathrm{G}
$$

is a compact symplectic manifold. The induced symplectic form will be denoted by $\bar{\omega}$. The equivariant homology class $[u] \in H_{2}\left(M_{\mathrm{G}} ; \mathbb{Z}\right)$ is defined by the following diagram, which also shows how it is related to the characteristic class $[P] \in H_{2}(\mathrm{BG} ; \mathbb{Z})$ and to the class $[\bar{u}] \in H_{2}(\bar{M} ; \mathbb{Z})$ in the case $\mu \circ u \equiv 0$. Note that, since $\mathrm{G}$ is connected, the class $[u]$ determines the isomorphism class of the bundle $P$. We denote $M_{\mathrm{G}}:=M \times_{\mathrm{G}}$ EG.

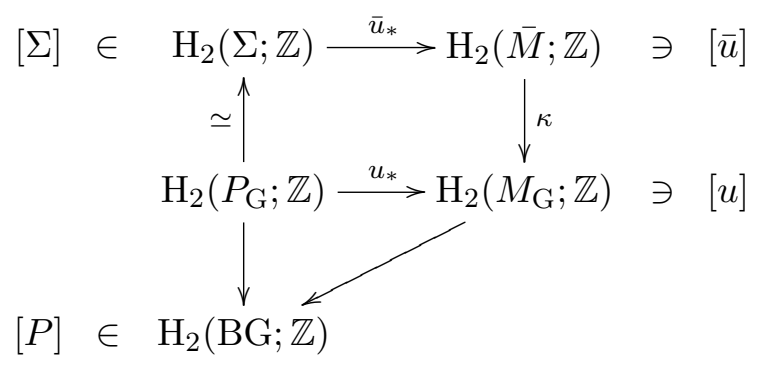


Fix a homology class $\bar{B} \in H_{2}(\bar{M} ; \mathbb{Z})$, let $B:=\kappa(\bar{B}) \in H_{2}\left(M_{\mathrm{G}} ; \mathbb{Z}\right)$, and denote the space of solutions of equations (1.1) that represent this homology class by

$$
\widetilde{\mathcal{M}}_{B, \Sigma}:=\left\{(u, A) \in C_{\mathrm{G}}^{\infty}(P, M) \times \mathcal{A}(P) \mid[u]=B, u \text { and } A \text { satisfy }(1.1)\right\} .
$$

Here $P \rightarrow \Sigma$ denotes a principal G-bundle whose characteristic class $[P] \in$ $H_{2}(\mathrm{BG} ; \mathbb{Z})$ is determined by $B$ as above. The quotient by the action of the gauge group will be denoted by

$$
\mathcal{M}_{B, \Sigma}:=\widetilde{\mathcal{M}}_{B, \Sigma} / \mathcal{G} \text {. }
$$

We impose another hypothesis which guarantees compactness [2].

(H2): There is a G-invariant almost complex structure $J \in \mathcal{J}_{\mathrm{G}}(M, \omega)$, a proper G-invariant function $f: M \rightarrow[0, \infty)$, and a constant $c>0$ such that

$$
f(x) \geq c \Longrightarrow\left\langle\nabla_{\xi} \nabla f(x), \xi\right\rangle+\left\langle\nabla_{J \xi} \nabla f(x), J \xi\right\rangle \geq 0
$$

for every nonzero vector $\xi \in T_{x} M$ and

$$
f(x) \geq c \Longrightarrow d f(x) J X_{\mu(x)}(x) \geq 0 .
$$

Moreover, $\int_{S^{2}} v^{*} \omega=0$ for every smooth map $v: S^{2} \rightarrow M$.

This hypothesis implies that $\sup _{P}(f \circ u) \leq c$ for every solution $(u, A)$ of equations (1.1) over any Riemann surface and in any homology class (see [2]). In $[\mathbf{2}]$, it is shown that the moduli space $\mathcal{M}_{B, \Sigma}$ is a smooth compact manifold of dimension

$$
\operatorname{dim} \mathcal{M}_{B, \Sigma}=\left(\frac{1}{2} \operatorname{dim} M-\operatorname{dim} \mathrm{G}\right) \chi(\Sigma)+2\left\langle c_{1}^{\mathrm{G}}(T M), B\right\rangle
$$

for a generic $J$, provided that hypotheses (H1) and (H2) are satisfied, $B$ is a nontorsion homology class, and the area of $\Sigma$ is sufficiently large. The latter condition, together with the energy identity, guarantees that every solution of equations (1.1) is somewhere close to the zero set of the moment map. The class $c_{1}^{\mathrm{G}}(T M) \in H^{2}\left(M_{\mathrm{G}} ; \mathbb{Z}\right)$ in the dimension formula denotes the equivariant first Chern class of the complex vector bundle $(T M, J)$.

\section{Examples.}

(i) In $[3]$, it is shown that (H2) follows from (H1) in the case of linear actions on $\mathbb{C}^{n}$.

(ii) The completion of a symplectically aspherical G-manifold $M$ with contact boundary satisfies (H2) whenever it admits a Liouville vector field $X$ near $\partial M$ satisfying $\omega\left(X_{\mu(x)}, X(x)\right) \geq 0$ for every $x \in \partial M$ (see $[\mathbf{2}$, Example 2.9]). A special case is an action of $\mathrm{G}$ on a cotangent bundle $T^{*} S$ that arises from an action on $S$. Namely, if $\mathfrak{g} \rightarrow \operatorname{Vect}(S): \xi \mapsto v_{\xi}$ is the infinitesimal action on $S$ then a moment map of the action on 
$T^{*} S$ is $\langle\mu(x, y), \xi\rangle=\left\langle y, v_{\xi}(x)\right\rangle$ for $y \in T_{x}^{*} S$ and hence the Liouville vector field $X(x, y)=(0, y)$ satisfies $\omega\left(X_{\mu(x, y)}, X(x, y)\right)=\left\langle y, v_{\mu(x, y)}(x)\right\rangle=$ $|\mu(x, y)|^{2} \geq 0$.

(iii) Let $M=E$ be the total space of a complex vector bundle $\pi: E \rightarrow S$ over a compact symplectically aspherical Kähler manifold $S$, equipped with a Hermitian structure, a unitary G-action, and a Hermitian connection $\nabla$ that preserves the G-action. Suppose that the endomorphism $i F^{\nabla}(v, J v)$ is negative semidefinite for every $v \in T S$. Then $M$ admits a G-invariant symplectic structure satisfying (H2) (see [2, Example 2.10]).

Consider the evaluation map ev $: \mathcal{M}_{B, \Sigma} \rightarrow M_{\mathrm{G}}$, defined by

$$
\operatorname{ev}_{G}([u, A]):=\left[u\left(p_{0}\right), \Theta_{0}(u, A)\right],
$$

where $p_{0} \in P$ is fixed and $\Theta_{0}: \widetilde{\mathcal{M}}_{B, \Sigma} \rightarrow$ EG is a smooth map such that

$$
\Theta_{0}\left(g^{-1} u, g^{*} A\right)=g\left(p_{0}\right)^{-1} \Theta_{0}(u, A) .
$$

This means that $\Theta_{0}$ is a classifying map for the principal G-bundle $\mathcal{P}_{B, \Sigma} \rightarrow$ $\mathcal{M}_{B, \Sigma}$ obtained as the quotient of $\widetilde{\mathcal{M}}_{B, \Sigma}$ by the based gauge group

$$
\mathcal{G}_{0}:=\left\{g \in \mathcal{G} \mid g\left(p_{0}\right)=\mathbb{1}\right\} .
$$

Let $\alpha \in H^{*}\left(M_{\mathrm{G}} ; \mathbb{Z}\right)$ be a class of degree $\operatorname{deg}(\alpha)=\operatorname{dim} \mathcal{M}_{B, \Sigma}$ and define

$$
\Phi_{B, \Sigma}(\alpha):=\int_{\mathcal{M}_{B, \Sigma}} \mathrm{ev}_{\mathrm{G}}^{*} \alpha .
$$

In [2], it is shown that this integer is independent of the almost complex structure $J$, the metric on $\Sigma$, and the point $p_{0}$ used to define it.

Now let $D:=\{z \in \mathbb{C}|| z \mid \leq 1\}$ and consider the space of maps $v: D \rightarrow M$ that map the boundary $\partial D$ to a G-orbit in $\mu^{-1}(0)$ :

$$
\mathcal{V}:=\left\{v: D \rightarrow M \mid \exists g: \mathbb{R} / 2 \pi \mathbb{Z} \rightarrow \mathrm{G} \exists x \in \mu^{-1}(0) \forall \theta \in \mathbb{R} \quad v\left(e^{i \theta}\right)=g(\theta) x\right\} .
$$

Let $\mathrm{m}: \mathcal{V} \rightarrow \mathbb{Z}$ denote the function which assigns to each element $v \in \mathcal{V}$ the Maslov index of the loop of symplectic matrices obtained from the linear maps $g(\theta): T_{x} M \rightarrow T_{g(\theta) x} M$ in a trivialization along $v$. Every smooth map $\bar{v}: S^{2} \rightarrow \bar{M}$ lifts to a map $v \in \mathcal{V}$ and in this case the Maslov index $\mathrm{m}(v)$ is equal to the first Chern number $\left\langle c_{1}(T \bar{M}), \bar{v}_{*}\left[S^{2}\right]\right\rangle$. The minimal Maslov number will be denoted by

$$
N:=\inf _{v \in \mathcal{V}, \operatorname{m}(v)>0} \mathrm{~m}(v) .
$$

This is a lower bound for the minimal Chern number of $\bar{M}$. We impose a third hypothesis. 
(H3): There exists a constant $\tau>0$ such that

$$
\int_{D} v^{*} \omega=\tau \mathrm{m}(v)
$$

for every $v \in \mathcal{V}$.

This hypothesis implies that the quotient $\bar{M}$ is a monotone symplectic manifold and that the energy of every holomorphic sphere in $\bar{M}$ is an integer multiple of $\hbar:=\tau N$. The main result of this paper asserts that under hypotheses (H1) to (H3) the invariant $\Phi_{B, S^{2}}$ agrees with the corresponding genus zero Gromov-Witten invariant of $\bar{M}$, provided that the cohomology classes $\alpha_{i}$ have degrees less than $2 N$.

Theorem 1.1. Assume (H1)-(H3) and let $\bar{B} \in H_{2}(\bar{M} ; \mathbb{Z})$ and $\alpha_{i} \in$ $H_{\mathrm{G}}^{*}(M ; \mathbb{Z})$ be given such that $\operatorname{deg}\left(\alpha_{i}\right)<2 N$ for $i=1, \ldots, k$ and

$$
\sum_{i=1}^{k} \operatorname{deg}\left(\alpha_{i}\right)=\left(\frac{1}{2} \operatorname{dim} M-\operatorname{dim} \mathrm{G}\right) \chi(\Sigma)+2\left\langle c_{1}^{\mathrm{G}}(T M), B\right\rangle,
$$

where $B:=\kappa(\bar{B}) \in H_{2}^{\mathrm{G}}(M ; \mathbb{Z})$. Then

$$
\Phi_{B, S^{2}}\left(\alpha_{1} \smile \cdots \smile \alpha_{k}\right)=\mathrm{GW}_{\bar{B}, S^{2}}\left(\bar{\alpha}_{1}, \ldots, \bar{\alpha}_{k}\right),
$$

where $\bar{\alpha}_{i}:=\kappa\left(\alpha_{i}\right) \in H^{*}(\bar{M} ; \mathbb{Z})$. If $[\omega-\mu]=\tau c_{1}^{\mathrm{G}}(T M)$ then the assertion continues to hold for Riemann surfaces $\Sigma$ of arbitrary genus.

\section{Remarks.}

(i) In the definition of $\Phi_{B, \Sigma}$ the complex structure on $\Sigma$ and the point $p_{0} \in P$ at which the map $u$ is evaluated are fixed and the cohomology class $\operatorname{ev}_{\mathrm{G}}^{*} \alpha \in H^{*}\left(\mathcal{M}_{B, \Sigma} ; \mathbb{Z}\right)$ is independent of the choice of complex structure on $\Sigma$ and the point $p_{0}$ used in the definition of $\mathrm{ev}_{\mathrm{G}}$. The Gromov-Witten invariants in Theorem 1.1 are also to be understood with a fixed complex structure on $\Sigma$ and with fixed marked points in the definitions of the evaluation maps, and with almost complex structures that are allowed to depend on the base point $z \in S^{2}$.

(ii) The assertion of Theorem 1.1 does not continue to hold in the case $\operatorname{deg}\left(\alpha_{i}\right) \geq 2 N$. For example, consider the standard action of $S^{1}$ on $\mathbb{C}^{n}$, let $P \rightarrow S^{2}$ be an $S^{1}$-bundle of degree $d \geq 0$, and denote by $c \in$ $H^{2}\left(\mathrm{~B} S^{1} ; \mathbb{Z}\right)=H_{S^{1}}^{2}\left(\mathbb{C}^{n} ; \mathbb{Z}\right)$ the positive generator. Then the minimal Chern number is $N=n$, the dimension of $\mathcal{M}_{d, S^{2}}$ is $2 n d+2 n-2$, and we have $\Phi_{d, S^{2}}\left(c^{m}\right)=1$ whenever $m=n d+n-1$. The corresponding Gromov-Witten invariant (for a $k$-tuple of classes $c^{m_{1}}, \ldots, c^{m_{k}}$ with $\left.m_{1}+\cdots+m_{k}=m\right)$ counts holomorphic spheres of degree $d$ in $\mathbb{C} P^{n-1}$ passing at $k$ given points $z_{1}, \ldots, z_{k} \in S^{2}$ through generic copies of $\mathbb{C} P^{n-1-m_{i}}$ for $i=1, \ldots, k$. Thus the Gromov-Witten invariant is zero whenever $\operatorname{deg}\left(c^{m_{i}}\right)=2 m_{i} \geq 2 n$ for some $i$. 
(iii) The condition $\operatorname{deg}\left(\alpha_{i}\right)<2 N$ in Theorem 1.1 leads to simplified bubbling phenomena which do not require an analysis of bubble trees. Namely, if we have a sequence of $\bar{J}$-holomorphic curves in $\bar{M}$ passing through submanifolds dual to the $\bar{\alpha}_{i}$ at the marked points $z_{i}$, and a holomorphic sphere bubbles off at a marked point $z_{j}$, then the limit curve (assumed to be in general position) cannot satisfy the remaining marked point conditions, for dimensional reasons. A similar phenomenon occurs in the adiabatic limit $\varepsilon \rightarrow 0$ explained below.

Equivariant and quantum cohomology. Assume $\bar{M}$ is monotone. Additively, the quantum cohomology $\mathrm{QH}^{*}(\bar{M})$ is the cohomology of $\bar{M}$ with coefficients in the group ring of $H_{2}(\bar{M} ; \mathbb{Z})$. Write an element of $\mathrm{QH}^{*}(\bar{M})$ as a finite sum

$$
\bar{\alpha}=\sum_{\bar{B} \in H_{2}(\bar{M} ; \mathbb{Z})} \bar{\alpha}_{\bar{B}} e^{\bar{B}}
$$

where $\bar{\alpha}_{\bar{B}} \in H^{*}(\bar{M} ; \mathbb{R})$ and

$$
\operatorname{deg}\left(e^{\bar{B}}\right):=2\left\langle c_{1}(T \bar{M}), \bar{B}\right\rangle .
$$

Choose an integral basis $\bar{e}_{0}, \ldots, \bar{e}_{n}$ of $H^{*}(\bar{M} ; \mathbb{R})$ and let $\bar{e}_{i}^{*}$ denote the dual basis in the sense that $\int_{\bar{M}} \bar{e}_{i} \smile \bar{e}_{j}^{*}=\delta_{i j}$. Then the ring structure on $\mathrm{QH}^{*}(\bar{M})$ is defined by

$$
\bar{\alpha}_{1} * \bar{\alpha}_{2}:=\sum_{\bar{B}_{1}, \bar{B}_{2}, \bar{B}} \sum_{i=0}^{n} \mathrm{GW}_{\bar{B}-\bar{B}_{1}-\bar{B}_{2}, S^{2}}\left(\bar{\alpha}_{1 \bar{B}_{1}}, \bar{\alpha}_{2 \bar{B}_{2}}, \bar{e}_{i}^{*}\right) \bar{e}_{i} e^{\bar{B}} .
$$

The sum is over all quadruples $i, \bar{B}_{1}, \bar{B}_{2}, \bar{B}$ satisfying $\operatorname{deg}\left(\bar{\alpha}_{1}\right)+\operatorname{deg}\left(\bar{\alpha}_{2}\right)=$ $\operatorname{deg}\left(\bar{e}_{i}\right)+2\left\langle c_{1}(T \bar{M}), \bar{B}\right\rangle$.

The Gromov-Witten invariant associated to a Riemann surface $\Sigma$, with a fixed complex structure $j_{\Sigma}$ and fixed marked points $z_{1}, \ldots, z_{k}$, can be extended to a map $\mathrm{GW}_{\bar{B}, \Sigma}: \mathrm{QH}^{*}(\bar{M}) \otimes \cdots \otimes \mathrm{QH}^{*}(\bar{M}) \rightarrow \mathbb{Z}$ by the formula

$$
\mathrm{GW}_{\bar{B}, \Sigma}\left(\bar{\alpha}_{1}, \ldots, \bar{\alpha}_{k}\right):=\sum_{\bar{B}_{i}} \mathrm{GW}_{\bar{B}-\bar{B}_{1}-\cdots-\bar{B}_{k}, \Sigma}\left(\bar{\alpha}_{1 \bar{B}_{1}}, \ldots, \bar{\alpha}_{k \bar{B}_{k}}\right) .
$$

With this convention the gluing formula for the Gromov-Witten invariants can be expressed in the form

$$
\mathrm{GW}_{\bar{B}, \Sigma}\left(\bar{\alpha}_{1}, \ldots, \bar{\alpha}_{k}\right)=\mathrm{GW}_{\bar{B}, \Sigma}\left(\bar{\alpha}_{1} * \cdots * \bar{\alpha}_{k}\right)
$$

(see [11, Exercise 11.1.14]).

Corollary 1.2. Assume $(\mathrm{H} 1-\mathrm{H} 3)$ and suppose that $H^{*}\left(M_{\mathrm{G}} ; \mathbb{R}\right)$ is generated by classes of degrees less than $2 N$. Then there exists a unique ring homomorphism $\varphi: H^{*}\left(M_{\mathrm{G}} ; \mathbb{R}\right) \longrightarrow \mathrm{QH}^{*}(\bar{M})$ such that

$$
\operatorname{deg}(\alpha)<2 N \quad \Longrightarrow \quad \varphi(\alpha)=\kappa(\alpha)
$$


for every $\alpha \in H^{*}\left(M_{\mathrm{G}} ; \mathbb{R}\right)$. This ring homomorphism satisfies

$$
\Phi_{\kappa(\bar{B}), S^{2}}(\alpha)=\mathrm{GW}_{\bar{B}, S^{2}}(\varphi(\alpha))
$$

for every $\alpha \in H^{*}\left(M_{\mathrm{G}} ; \mathbb{R}\right)$ and every $\bar{B} \in H_{2}(\bar{M} ; \mathbb{Z})$.

Proof. Let $\alpha \in H^{*}\left(M_{\mathrm{G}} ; \mathbb{R}\right)$ and choose $\alpha_{i j} \in H^{*}\left(M_{\mathrm{G}} ; \mathbb{R}\right)$ such that

$$
\alpha=\sum_{i=1}^{k} \alpha_{i 1} \smile \cdots \smile \alpha_{i \ell}, \quad \operatorname{deg}\left(\alpha_{i j}\right)<2 N .
$$

Define

$$
\varphi(\alpha):=\sum_{i=1}^{k} \kappa\left(\alpha_{i 1}\right) * \cdots * \kappa\left(\alpha_{i \ell}\right) .
$$

We prove that $\varphi(\alpha)$ is independent of the choice of $\alpha_{i j}$. To see this, note that, since the cohomology of $\bar{M}$ is generated by classes of degree less than $2 N$, so is the quantum cohomology. This means that a quantum cohomology class $\bar{\alpha} \in \mathrm{QH}^{*}(\bar{M})$ is zero if and only if $\mathrm{GW}_{\bar{B}, S^{2}}\left(\bar{\alpha}, \bar{\beta}_{1}, \ldots, \bar{\beta}_{m}\right)=0$ for every $\bar{B} \in H_{2}(\bar{M} ; \mathbb{Z})$ and all $\bar{\beta}_{1}, \ldots, \bar{\beta}_{m} \in H^{*}(\bar{M} ; \mathbb{R})$ such that $\operatorname{deg}\left(\bar{\beta}_{j}\right)<$ $2 N$ for all $j$. Now suppose that the expression on the right-hand side of equation (1.4) is nonzero. Then, by what we have just observed, there exist cohomology classes $\bar{\beta}_{1}, \ldots, \bar{\beta}_{m}$ of degrees less than $2 N$ and a homology class $\bar{B} \in H_{2}(\bar{M} ; \mathbb{Z})$ such that

$$
\sum_{i=1}^{k} \mathrm{GW}_{\bar{B}, S^{2}}\left(\kappa\left(\alpha_{i 1}\right), \ldots, \kappa\left(\alpha_{i \ell}\right), \bar{\beta}_{1}, \ldots, \bar{\beta}_{m}\right) \neq 0 .
$$

Since the homomorphism $\kappa: H^{*}\left(M_{\mathrm{G}} ; \mathbb{R}\right) \rightarrow H^{*}(\bar{M} ; \mathbb{R})$ is surjective (cf. [10]), there exist classes $\beta_{j} \in H^{*}\left(M_{\mathrm{G}} ; \mathbb{R}\right.$ ) (of degrees less than $2 N$ ) such that $\kappa\left(\beta_{j}\right)=\bar{\beta}_{j}$ for every $j$. Hence, by Theorem 1.1,

$$
\sum_{i=1}^{k} \Phi_{\kappa(\bar{B}), S^{2}}\left(\alpha_{i 1} \smile \cdots \smile \alpha_{i \ell} \smile \beta_{1} \smile \cdots \smile \beta_{m}\right) \neq 0,
$$

and hence $\alpha \neq 0$. This shows that $\varphi$ is well defined. The map $\varphi$ is obviously a ring homomorphism. The formula $\Phi_{B, S^{2}}(\alpha)=\mathrm{GW}_{\bar{B}, S^{2}}(\varphi(\alpha))$ follows immediately from Theorem 1.1 and the gluing formula (1.2) for the GromovWitten invariants.

Remark 1.3. Under our assumptions, both the Gromov-Witten and the vortex invariants take integer (not rational) values. Hence the assertion of Corollary 1.2 continues to hold for (quantum) cohomology groups with coefficients in $\mathbb{Z}$ or $\mathbb{Z}_{2}$, for example. 
Remark 1.4. Let $\mathcal{R}$ denote the group ring of $H_{2}(\bar{M} ; \mathbb{Z})$. Then $\mathrm{QH}^{*}(\bar{M})$ is an $\mathcal{R}$-module and the ring structure is compatible with the $\mathcal{R}$-module structure. Hence the ring homomorphism of Corollary 1.2 extends uniquely to a homomorphism

$$
\varphi: H^{*}\left(M_{\mathrm{G}} ; \mathcal{R}\right) \rightarrow \mathrm{QH}^{*}(\bar{M})
$$

of rings and $\mathcal{R}$-modules. This extended homomorphism is surjective. This follows, by an easy induction argument, from the surjectivity of Kirwan's homomorphism to the ordinary cohomology of $\bar{M}$.

Remark 1.5. The homomorphism $\varphi: H^{*}\left(M_{\mathrm{G}}\right) \rightarrow \mathrm{QH}^{*}(\bar{M})$ can be defined geometrically in terms of the vortex equations over $\mathbb{C}$ :

$$
\partial_{s} u+L_{u} \Phi+J\left(\partial_{t} u+L_{u} \Psi\right)=0, \quad \partial_{s} \Psi-\partial_{t} \Phi+[\Phi, \Psi]+\mu(u)=0 .
$$

Here $u: \mathbb{C} \rightarrow M$ and $\Phi, \Psi: \mathbb{C} \rightarrow \mathfrak{g}$ are smooth functions. Equation (1.5) is a special case of equations (11.1) with $\Sigma=\mathbb{C}$ and $A=\Phi \mathrm{d} s+\Psi \mathrm{d} t$. For every finite energy solution of equations (1.5) in radial gauge there is a loop $g: S^{1} \rightarrow \mathrm{G}$ and a point $x_{0} \in \mu^{-1}(0)$ such that

$$
\lim _{r \rightarrow \infty} u\left(r \mathrm{e}^{\mathrm{i} \theta}\right)=g\left(\mathrm{e}^{\mathrm{i} \theta}\right) x_{0}
$$

(see Section 11). Every map $u: \mathbb{C} \rightarrow M$ that satisfies equation (1.6) determines a class $B=[u] \in H_{2}\left(M_{\mathrm{G}} ; \mathbb{Z}\right)$. Now the moduli space $\mathcal{M}_{B}(J)$ of gauge equivalence classes of solutions of equations (1.5) and (1.6) that represent the class $B$ has two evaluation maps ev $0: \mathcal{M}_{B} \rightarrow M_{\mathrm{G}}$ and $\mathrm{ev}_{\infty}: \mathcal{M}_{B} \rightarrow \bar{M}$. The map $\varphi$ can be defined by

$$
\varphi(\alpha)=\sum_{i=0}^{n} \sum_{\bar{B}}\left(\int_{\mathcal{M}_{\kappa(\bar{B})}} \mathrm{ev}_{0}^{*} \alpha \smile \mathrm{ev}_{\infty}^{*} \bar{e}_{i}^{*}\right) \bar{e}_{i} e^{\bar{B}} .
$$

The details of this construction will be carried out elsewhere.

Outline of the proof of Theorem 1.1. The proof of Theorem 1.1 is based on an adiabatic limit argument in which the metric on the Riemann surface is scaled by a large factor $\varepsilon^{-2}$. Then equations (1.1) have the form

$$
\bar{\partial}_{J, A}(u)=0, \quad * F_{A}+\varepsilon^{-2} \mu(u)=0 .
$$

The solutions of (1.7) minimize the $\varepsilon$-dependent energy

$$
E^{\varepsilon}(u, A)=\frac{1}{2} \int_{\Sigma}\left(\left|d_{A} u\right|^{2}+\varepsilon^{2}\left|F_{A}\right|^{2}+\varepsilon^{-2}|\mu(u)|^{2}\right) \operatorname{dvol}_{\Sigma},
$$

and the value of this functional at a solution of equations (1.7) is independent of $\varepsilon$ in a given equivariant homology class. In this paper, we examine the limit behaviour of the solutions of equations (1.7), as $\varepsilon$ tends to zero for Riemann surfaces of any genus. The limit equations have the form

$$
\bar{\partial}_{J, A}(u)=0, \quad \mu(u)=0 .
$$


The solutions of equations (1.8) can be interpreted as pseudoholomorphic curves in the symplectic quotient $\bar{M}=\mu^{-1}(0) / \mathrm{G}$ with respect to the induced family of almost complex structures $\bar{J}_{z}$ (see Section 2). We impose a further hypothesis that is satisfied for a generic family of G-invariant almost complex structures on $M$ :

(H4): Every nonconstant $\bar{J}$-holomorphic curve $\bar{u}: \Sigma \rightarrow \bar{M}$ is regular, i.e., the linearized Cauchy-Riemann operator along $\bar{u}$ is surjective.

This hypothesis guarantees that the moduli space of holomorphic curves in $\bar{M}$ is smooth.

The proof of Theorem 1.1 requires three preliminary theorems which are of interest in their own rights. Theorem 3.1 constructs a $\mathcal{G}(P)$-equivariant map

$$
\left(u_{0}, A_{0}\right) \mapsto\left(u_{\varepsilon}, A_{\varepsilon}\right)=: \tilde{\mathcal{T}}^{\varepsilon}\left(u_{0}, A_{0}\right),
$$

which assigns to every regular solution of equation (1.8) a nearby solution of equations (1.7) for $\varepsilon$ sufficiently small. Theorem 3.2 shows that the map $\widetilde{\mathcal{T}}^{\varepsilon}$ constructed in Theorem 3.1 is locally surjective in the sense that every solution of equations (1.7) that is sufficiently close to a solution $\left(u_{0}, A_{0}\right)$ of equation (1.8) must be in the image of $\widetilde{\mathcal{T}}^{\varepsilon}$. The neighbourhood in which surjectivity holds depends on $\varepsilon$ : it becomes smaller as $\varepsilon$ tends to zero. Theorem 3.3 strengthens the local surjectivity result of Theorem 3.2. We remove the assumption that the solution of equations (1.7) is close to some given solution of equation (1.8). However, we consider only solutions of equations (1.7) that satisfy a suitable $L^{\infty}$-bound on the first derivatives and prove that every solution of equations (1.7) that satisfies this bound lies in the image of $\widetilde{\mathcal{T}}^{\varepsilon}$ for $\varepsilon$ small. The proof of Theorem 1.1 is then based on a bubbling argument in the small $\varepsilon$ limit which establishes a one-to-one correspondence between the solutions of equation (1.8) and those of equations (1.7) in a zero-dimensional setting, where additional conditions have been imposed.

In Section 2, we review standard results about the moduli space of pseudoholomorphic curves in the symplectic quotient $M / / \mathrm{G}$ and rephrase them in terms of solutions of equation (1.8). Theorems 3.1 to 3.3 will be stated in Section 3. The remaining sections are devoted to the proofs of the four main theorems.

The general outline of the proof of Theorem 1.1 is analogous to the proof of the Atiyah-Floer conjecture in $[\mathbf{5}, \mathbf{6}]$; there are several new ingredients.

In Sections 4 and 5, we establish the linear and quadratic estimates needed for the construction of the map $\mathcal{T}^{\varepsilon}$; this construction is carried out in Section 6 where Theorem 3.1 is proved. In contrast to [6] (where the relevant moduli space is a finite set), care must be taken to establish that the constants in the estimates depend continuously on the point in $\mathcal{M}^{0}$ and we must control the second instead of just the first derivatives. 
In Section 7, we establish an $\varepsilon$-local slice theorem and in Section 8 we construct a tubular neighbourhood of $\mathcal{M}^{\varepsilon}$ and carry out the proof of Theorem 3.2. The $\varepsilon$-local slice theorem is analogous to [6, Proposition 6.2]. The tubular neighbourhood theorem for $\mathcal{M}^{\varepsilon}$ involves an estimate for the derivative of the map given by the $\varepsilon$-local slice theorem; this is where the estimates for the $(2, p, \varepsilon)$-norms are needed. As a result, the entire adiabatic limit argument has to be carried out for these higher norms. In contrast, the analogous result in [6, Proposition 6.3] can be disposed of with a simple time shift argument.

In Section 9, we prove an a priori estimate which asserts that every solution of equations (1.7) that satisfies a certain $L^{\infty}$ bound must be $\varepsilon^{2}$-close to the zero set of the moment map. As a consequence, we obtain in Theorem 3.3 a strong local surjectivity result for the map $\mathcal{T}^{\varepsilon}$, which is proved in Section $10 .^{1}$

In Section 11, we establish the asymptotic behaviour and the quantization of the energy for solutions of the nonlinear vortex equations on the complex plane. In Section 12, we construct a classifying map on an open set in $C_{\mathrm{G}}^{\infty}(P, M) \times \mathcal{A}(P)$, which contains the moduli spaces $\mathcal{M}^{\varepsilon}$ for all $\varepsilon \in\left[0, \varepsilon_{0}\right]$, with values in a finite-dimensional approximation of EG; we also establish $C^{1}$-convergence for the evaluation map and prove Theorem 1.1.

\section{Pseudoholomorphic curves}

For $z \in \Sigma$, let $\bar{J}_{z}$ denote the almost complex structure on $\bar{M}$ induced by $J_{z}$, let $\bar{P} \longrightarrow \bar{M}$ denote the principal G-bundle $\bar{P}:=\mu^{-1}(0) \subset M$, and let $\bar{A}$ denote the connection on $\bar{P}$ determined by $\omega$ and $J$. If $(u, A)$ is a solution of equation (1.8), then $u$ descends to a $\bar{J}$-holomorphic curve $\bar{u}: \Sigma \rightarrow \bar{M}$ and $A$ is the pullback of $\bar{A}$ under $\bar{u}$. Two gauge equivalent solutions descend to the same map $\bar{u}$ and every $\bar{J}$-holomorphic curve $\bar{u}: \Sigma \rightarrow \bar{M}$ lifts to a solution of equation (1.8) for some principal G-bundle $P$ (isomorphic to the pullback of $\bar{P}$ under $\bar{u}$ ).

Fix a homology class $\bar{B} \in H_{2}(\bar{M} ; \mathbb{Z})$, let $B:=\kappa(\bar{B}) \in H_{2}^{\mathrm{G}}(M ; \mathbb{Z})$, and consider the space

$$
\widetilde{\mathcal{M}}_{B, \Sigma}^{0}:=\left\{(u, A) \in C_{\mathrm{G}}^{\infty}(P, M) \times \mathcal{A}(P) \mid[u]=B \text { and (1.8) holds }\right\} .
$$

This space is invariant under the action of the gauge group $\mathcal{G}(P)$. Under our standing hypothesis (H4), the quotient

$$
\mathcal{M}_{B, \Sigma}^{0}:=\widetilde{\mathcal{M}}_{B, \Sigma}^{0} / \mathcal{G}(P)
$$

\footnotetext{
${ }^{1}$ Compare this with [6, Theorem 8.1]; the strenghthened form of [6, Theorem 8.1] is needed to close a gap in the bubbling argument in the proof of [6, Theorem 9.1].
} 
is a smooth manifold of dimension

$$
\operatorname{dim} \mathcal{M}_{B, \Sigma}^{0}=\left(\frac{1}{2} \operatorname{dim} M-\operatorname{dim} \mathrm{G}\right) \chi(\Sigma)+2\left\langle c_{1}^{\mathrm{G}}(T M), B\right\rangle
$$

(see [11, Theorem 3.3.4]). Note that $\mathcal{M}_{B, \Sigma}$ and $\mathcal{M}_{B, \Sigma}^{0}$ have the same dimension.

For later reference, we now introduce explicit notation for a local parametrization of $\mathcal{M}_{B, \Sigma}^{0}$ by the kernel of the linearized operator. Linearizing equations (1.8) at a solution $(u, A)$ gives rise to the Cauchy-Riemann operator

given by

$$
\mathcal{D}^{0}:=\mathcal{D}_{(u, A)}^{0}: \Omega^{0}\left(\Sigma, H_{u}\right) \rightarrow \Omega^{0,1}\left(\Sigma, H_{u}\right)
$$

$$
\mathcal{D}^{0} \xi_{0}:=\pi_{u} D_{(u, A)} \xi_{0}
$$

where $D_{(u, A)}: \Omega^{0}\left(\Sigma, u^{*} T M / \mathrm{G}\right) \rightarrow \Omega^{0,1}\left(u^{*} T M / \mathrm{G}\right)$ is the operator (B.3) in Appendix B. The bundle $H_{u} \rightarrow \Sigma$ and the projection $\pi_{u}: u^{*} T M \rightarrow H_{u}$ are defined as follows. Consider the bundle $H \longrightarrow \Sigma \times \mu^{-1}(0)$ with fibres

$$
H_{z, x}:=\operatorname{ker} d \mu(x) \cap \operatorname{ker} d \mu(x) J_{z} .
$$

There is an orthogonal decomposition

$$
T_{x} M=\operatorname{im} L_{x} \oplus H_{z, x} \oplus \operatorname{im} J_{z} L_{x}
$$

for every $(z, x) \in \Sigma \times \mu^{-1}(0)$, where $L_{x}: \mathfrak{g} \rightarrow T_{x} M$ the infinitesimal action, i.e.,

$$
L_{x} \eta:=X_{\eta}(x) .
$$

Its dual operator with respect to the metric determined by $J_{z}$ is given by

$$
L_{x}^{*}=L_{x}^{*_{z}}=d \mu(x) J_{z}(x) .
$$

Now let $u: P \rightarrow \mu^{-1}(0)$ be an equivariant map and consider the pullback of $H$ under the map $\tilde{u}: P \rightarrow \Sigma \times \mu^{-1}(0)$, given by $\tilde{u}(p):=(\pi(p), u(p))$. This pullback is a G-equivariant vector bundle over $P$ and its quotient

$$
H_{u}:=\tilde{u}^{*} H / \mathrm{G} \longrightarrow \Sigma
$$

is naturally isomorphic to the pullback of the tangent bundle $T \bar{M}$ under the induced map $\bar{u}: \Sigma \rightarrow \bar{M}$. Let $\pi_{u}: u^{*} T M / \mathrm{G} \rightarrow H_{u}$ denote the orthogonal projection onto the harmonic part. Thus $\pi_{u}[\xi]:=\left[\pi_{u} \xi\right]$ where the lifted projection $u^{*} T M \rightarrow u^{*} H$ (also denoted by $\pi_{u}$ ) is given by

$$
\pi_{u} \xi:=\xi-L_{u}\left(L_{u}^{*} L_{u}\right)^{-1} L_{u}^{*} \xi+J L_{u}\left(L_{u}^{*} L_{u}\right)^{-1} L_{u}^{*} J \xi
$$

for a G-equivariant section $\xi: P \rightarrow u^{*} T M$.

Theorem 2.1. Assume (H1) and (H4) and fix a constant $p>2$. For every $\left(\bar{u}_{0}, \bar{A}_{0}\right) \in \widetilde{\mathcal{M}}_{B, \Sigma}^{0}$ there exist a sequence of positive constants $\delta, c, c_{1}, c_{2}, \ldots$ and a map

$$
\mathcal{F}^{0}:=\mathcal{F}_{\left(\bar{u}_{0}, \bar{A}_{0}\right)}^{0}: B_{\delta}^{0} \rightarrow \widetilde{\mathcal{M}}_{B, \Sigma}^{0}, \quad B_{\delta}^{0}:=\left\{\xi_{0} \in \operatorname{ker} \mathcal{D}_{\left(\bar{u}_{0}, \bar{A}_{0}\right)}^{0} \mid\left\|\xi_{0}\right\|_{L^{p}}<\delta\right\}
$$


such that the following holds.

(i) If $\xi_{0} \in B_{\delta}^{0}$ then there exists a unique pair of sections $\xi_{1} \in \Omega^{0}\left(\Sigma, H_{\bar{u}_{0}}\right)$ and $\xi_{2} \in \Omega^{0}\left(\Sigma, \mathrm{im} J L_{\bar{u}_{0}} / \mathrm{G}\right)$ such that

$$
\xi_{1} \in \operatorname{im}\left(\mathcal{D}_{\left(\bar{u}_{0}, \bar{A}_{0}\right)}^{0}\right)^{*}, \quad\left\|\xi_{1}\right\|_{W^{1, p}}+\left\|\xi_{2}\right\|_{W^{1, p}} \leq c\left\|\xi_{0}\right\|_{W^{1, p}}
$$

and the pair $\left(u_{0}, A_{0}\right)$, given by

$$
\begin{aligned}
& u_{0}:=\exp _{\bar{u}_{0}}^{\mu^{-1}(0)}\left(\xi_{0}+\xi_{1}\right)=\exp _{\bar{u}_{0}}\left(\xi_{0}+\xi_{1}+\xi_{2}\right), \\
& A_{0}:=-\left(L_{u_{0}}^{*} L_{u_{0}}\right)^{-1} L_{u_{0}}^{*} d u_{0},
\end{aligned}
$$

satisfies (1.8). The pair $\left(u_{0}, A_{0}\right)$ is the image of $\xi_{0}$ under $\mathcal{F}^{0}$.

(ii) For every integer $k \geq 1$ and every $\xi_{0} \in B_{\delta}^{0}$ we have

$$
\begin{aligned}
\left\|\xi_{1}\right\|_{W^{k, p}}+\left\|\xi_{2}\right\|_{W^{k, p}} & \leq c_{k}\left\|\xi_{0}\right\|_{W^{k, p}}^{2}, \\
\left\|A_{0}-\bar{A}_{0}\right\|_{W^{k, p}} & \leq c_{k}\left\|\xi_{0}\right\|_{W^{k, p}},
\end{aligned}
$$

where $\xi_{1}, \xi_{2}$, and $A_{0}$ are as in (i).

(iii) The map $\mathcal{F}^{0}$ is smooth and $d \mathcal{F}^{0}(0) \xi_{0}=\left(\xi_{0}, \alpha_{0}\right)$, where $\alpha_{0} \in \Omega^{1}\left(\Sigma, \mathfrak{g}_{P}\right)$ is uniquely determined by the equation

$$
D \bar{\partial}_{J, \bar{A}_{0}}\left(\bar{u}_{0}\right) \xi_{0}+X_{\alpha_{0}}\left(\bar{u}_{0}\right)^{0,1}=0 .
$$

This theorem 2.1 is a standard result in the theory of holomorphic curves (see, e.g. [11, Theorem 3.5.2]). It follows from Fredholm theory and an infinite-dimensional version of the implicit function theorem. In most applications, the moduli space $\mathcal{M}_{B, \Sigma}^{0}$ is not compact. However, it can be exhausted by the compact subsets

$$
\mathcal{M}_{B, \Sigma}^{0}\left(c_{0}\right):=\widetilde{\mathcal{M}}_{B, \Sigma}^{0}\left(c_{0}\right) / \mathcal{G}(P),
$$

where $c_{0}>0$ and

$$
\widetilde{\mathcal{M}}_{B, \Sigma}^{0}\left(c_{0}\right):=\left\{(u, A) \in \widetilde{\mathcal{M}}_{B, \Sigma}^{0} \mid\left\|d_{A} u\right\|_{L^{\infty}}+\left\|F_{A}\right\|_{L^{\infty}} \leq c_{0}\right\} .
$$

Note that $\widetilde{\mathcal{M}}_{B, \Sigma}^{0}\left(c_{0}\right)$ is invariant under the action of $\mathcal{G}(P)$. For later reference, we prove the following lemma.

Lemma 2.2. Fix a reference connection $\widehat{A} \in \mathcal{A}(P)$. Then, for every $c_{0}>0$ and every integer $\ell \in \mathbb{N}$, there exists a constant $c=c\left(c_{0}, \ell\right)>0$ such that, for every $\left(u_{0}, A_{0}\right) \in \widetilde{\mathcal{M}}_{B, \Sigma}^{0}\left(c_{0}\right)$, we have

$$
\inf _{g \in \mathcal{G}(P)}\left(\left\|g^{-1} u\right\|_{C^{\ell}}+\left\|g^{*} A_{0}-\widehat{A}\right\|_{C^{\ell}}\right) \leq c .
$$

Proof. Suppose, by contradiction, that there is a sequence $\left(u_{\nu}, A_{\nu}\right) \in$ $\widetilde{\mathcal{M}}_{B, \Sigma}^{0}\left(c_{0}\right)$ such that $\left\|g^{-1} u_{\nu}\right\|_{C^{\ell}}+\left\|g^{*} A_{\nu}-\widehat{A}\right\|_{C^{\ell}} \geq \nu$ for every $\nu$ and every $g \in \mathcal{G}(P)$. By [11, Theorem B.4.2], there exists a subsequence, still denoted by $\left(u_{\nu}, A_{\nu}\right)$, such that the induced maps $\bar{u}_{\nu}: \Sigma \rightarrow M / / \mathrm{G}$ converge in the 
$C^{\infty}$-topology to a smooth $\bar{J}$-holomorphic curve. The limit curve represents the same homotopy class as the approximating curves and hence can be represented by a pair $(u, A) \in \widetilde{\mathcal{M}}_{B, \Sigma}^{0}\left(c_{0}\right)$. Since the sequence $\bar{u}_{\nu}$ converges to $\bar{u}: \Sigma \rightarrow M / / \mathrm{G}$ in the $C^{\ell+1}$-topology, there exists a constant $\nu_{0} \geq 0$ such that, for every $\nu \geq \nu_{0}$, there exist a gauge transformation $g_{\nu}$ and a section $\xi_{\nu} \in \Omega^{0}\left(\Sigma, H_{u}\right)$ such that

$$
g_{\nu}^{-1} u_{\nu}=\exp _{u}\left(\xi_{\nu}\right), \quad \lim _{\nu \rightarrow \infty}\left\|\xi_{\nu}\right\|_{C^{\ell+1}}=0 .
$$

The formulae

$$
g_{\nu}^{*} A_{\nu}=-\left(L_{g_{\nu}^{-1} u_{\nu}}^{*} L_{g_{\nu}^{-1} u_{\nu}}\right)^{-1} L_{g_{\nu}^{-1} u_{\nu}} d\left(g_{\nu}^{-1} u_{\nu}\right), \quad A=-\left(L_{u}^{*} L_{u}\right)^{-1} L_{u} d u
$$

show that $g_{\nu}^{*}\left(u_{\nu}, A_{\nu}\right)$ converges to $(u, A)$ in the $C^{\ell}$ topology. This contradicts the choice of the sequence $\left(u_{\nu}, A_{\nu}\right)$ and hence proves the lemma.

Theorem 2.3. Assume $(H 1)$ and $(H 4)$, let $\bar{B} \in H_{2}(M ; \mathbb{Z})$ be a nontorsion homology class, and let $\left(\Sigma, \mathrm{dvol}_{\Sigma}, j_{\Sigma}\right)$ be a compact Riemann surface. Then, for every $c_{0}>0$ and every $p>2$, there exist positive constants $c$ and $\delta$ such that the following holds. If $\bar{u}: \Sigma \rightarrow \bar{M}$ is a smooth map such that $[\bar{u}]=\bar{B}$ and

$$
\|d \bar{u}\|_{L^{\infty}} \leq c_{0}, \quad\left\|\bar{\partial}_{\bar{J}}(\bar{u})\right\|_{L^{p}} \leq \delta
$$

then there exists a section $\bar{\xi} \in \Omega^{0}\left(\Sigma, \bar{u}^{*} T \bar{M}\right)$ such that

$$
\bar{\partial}_{\bar{J}}\left(\exp _{\bar{u}}(\bar{\xi})\right)=0, \quad\|\bar{\xi}\|_{W^{1, p}} \leq c\left\|\bar{\partial}_{\bar{J}}(\bar{u})\right\|_{L^{p}} .
$$

Proof. This is again a standard result for pseudoholomorphic curves and the proof is almost word by word the same as that of [6, Theorem 2.5]. Here is a sketch. One argues by contradiction. If the result were false, there would be a sequence of smooth maps $\bar{u}_{i}: \Sigma \rightarrow \bar{M}$ that satisfies

$$
\sup _{i}\left\|d \bar{u}_{i}\right\|_{L^{\infty}}<\infty, \quad \lim _{i \rightarrow \infty}\left\|\bar{\partial}_{\bar{J}}\left(\bar{u}_{i}\right)\right\|_{L^{p}}=0,
$$

but which does not satisfy the conclusion of the theorem for any constant $c$. This means that the $W^{1, p}$-distance of $\bar{u}_{i}$ to the space of $\bar{J}$-holomorphic curves is not controlled uniformly by the $L^{p}$-norm of $\bar{\partial}_{\bar{J}}\left(\bar{u}_{i}\right)$. Now, by the Arzéla-Ascoli and Banach-Alaoglu theorems, a suitable subsequence of $\bar{u}_{i}$ converges, strongly with respect to the sup-norm and weakly in $W^{1, p}$, to a $\bar{J}$-holomorphic curve $\bar{u}$. It follows from standard elliptic regularity for $\bar{J}$-holomorphic curves that $\bar{u}_{i}$ then converges strongly with respect to the $W^{1, p}$-norm. To see this, write $\bar{u}_{i}=\exp _{\bar{u}}\left(\bar{\xi}_{i}\right)$ and observe that

$$
\begin{aligned}
\left\|\bar{\xi}_{i}\right\|_{W^{1, p}} & \leq c_{1}\left(\left\|D_{\bar{u}} \bar{\xi}_{i}\right\|_{L^{p}}+\left\|\bar{\xi}_{i}\right\|_{L^{p}}\right) \\
& \leq c_{2}\left(\left\|\bar{\partial}_{\bar{J}}\left(\bar{u}_{i}\right)\right\|_{L^{p}}+\left\|\bar{\xi}_{i}\right\|_{W^{1, p}}\left\|\bar{\xi}_{i}\right\|_{L^{\infty}}\right)+c_{1}\left\|\bar{\xi}_{i}\right\|_{L^{p}} .
\end{aligned}
$$

Here the first inequality is the elliptic estimate for the Cauchy-Riemann operator $D_{\bar{u}}$ and the second is the quadratic estimate for $\bar{\partial}_{\bar{J}}$. With this established, it follows from hypothesis (H4) and the implicit function 
theorem for the operator $\bar{\partial}_{\bar{J}}$ that there exists a sequence of $\bar{J}$-holomorphic curves $\bar{u}_{0 i}$ whose $W^{1, p}$-distance to $\bar{u}_{i}$ is bounded above by a fixed constant times the $L^{p}$-norm of $\bar{\partial}_{\bar{J}}\left(\bar{u}_{i}\right)$ (see [6, Theorem 2.1]). This shows that the sequence $\bar{u}_{i}$ does after all satisfy the conclusion of the theorem, in contradiction to our assumption.

\section{Adiabatic limits}

Before stating our main results, we introduce some notation. Fix an equivariant homology class $\bar{B} \in H_{2}(\bar{M} ; \mathbb{Z})$, let $B:=\kappa(\bar{B})$, and denote

$$
\mathcal{B}:=\left\{(u, A) \in C_{\mathrm{G}}^{\infty}(P, M) \times \mathcal{A}(P) \mid[u]=B\right\} .
$$

This space is an infinite-dimensional Frêchet manifold with tangent space

$$
T_{(u, A)} \mathcal{B}:=\Omega^{0}\left(\Sigma, u^{*} T M / \mathrm{G}\right) \times \Omega^{1}\left(\Sigma, \mathfrak{g}_{P}\right) .
$$

It carries an action of the gauge group $\mathcal{G}=\mathcal{G}(P)$ by $g^{*}(u, A)=\left(g^{-1} u, g^{*} A\right)$. Consider the vector bundle $\mathcal{E} \rightarrow \mathcal{B}$ with fibres

$$
\mathcal{E}_{(u, A)}:=\Omega^{0,1}\left(\Sigma, u^{*} T M / \mathrm{G}\right) \oplus \Omega^{0}\left(\Sigma, \mathfrak{g}_{P}\right),
$$

and the $\mathcal{G}$-equivariant section $\mathcal{F}^{\varepsilon}: \mathcal{B} \rightarrow \mathcal{E}$ given by

$$
\mathcal{F}^{\varepsilon}(u, A):=\left(\bar{\partial}_{J, A}(u), * F_{A}+\varepsilon^{-2} \mu(u)\right) .
$$

The zero set of this section is the space

$$
\widetilde{\mathcal{M}}_{B, \Sigma}^{\varepsilon}:=\{(u, A) \in \mathcal{B} \mid u \text { and } A \text { satisfy }(1.7)\} .
$$

Its quotient by the action of the gauge group will be denoted by

$$
\mathcal{M}_{B, \Sigma}^{\varepsilon}:=\widetilde{\mathcal{M}}_{B, \Sigma}^{\varepsilon} / \mathcal{G}(P) .
$$

The following theorem asserts the existence of solutions of equations (1.7) for sufficiently small $\varepsilon$ near every regular solution of equation (1.8). The result is quantitative and the estimates are expressed in terms of suitable $\varepsilon$ dependent norms. Moreover, an operator $\mathcal{D}^{\varepsilon}: T_{(u, A)} \mathcal{B} \rightarrow \mathcal{E}_{(u, A)} \oplus \Omega^{0}\left(\Sigma, \mathfrak{g}_{P}\right)$ appears. This operator is the augmented vertical differential of $\mathcal{F}^{\varepsilon}$. The operator and the norms will be defined in Section 4 .

Theorem 3.1. Assume (H1) and $(H 4)$ and let $\bar{B} \in H_{2}(\bar{M} ; \mathbb{Z})$ be a nontorsion homology class. Then, for every $c_{0}>0$ and every $p>2$, there exist positive constants $\varepsilon_{0}, c$, and $\delta$ such that for every $\varepsilon \in\left(0, \varepsilon_{0}\right]$ there exists a $\mathcal{G}(P)$-equivariant map

$$
\widetilde{\mathcal{T}}^{\varepsilon}: \widetilde{\mathcal{M}}_{B, \Sigma}^{0}\left(c_{0}\right) \rightarrow \widetilde{\mathcal{M}}_{B, \Sigma}^{\varepsilon}
$$

that satisfies the following conditions. 
(a) If $\left(u_{0}, A_{0}\right) \in \widetilde{\mathcal{M}}_{B, \Sigma}^{0}\left(c_{0}\right)$ then $\widetilde{\mathcal{T}}^{\varepsilon}\left(u_{0}, A_{0}\right)=\left(\exp _{u_{0}}\left(\xi_{\varepsilon}\right), A_{0}+\alpha_{\varepsilon}\right)$, where $\zeta_{\varepsilon}=\left(\xi_{\varepsilon}, \alpha_{\varepsilon}\right) \in T_{\left(u_{0}, A_{0}\right)} \mathcal{B}$ satisfies

$$
\begin{aligned}
\left\|\zeta_{\varepsilon}\right\|_{2, p, \varepsilon ;\left(u_{0}, A_{0}\right)} & \leq c \varepsilon^{2}, \quad-d_{A_{0}}^{*} \alpha_{\varepsilon}+\varepsilon^{-2} L_{u_{0}}^{*} \xi_{\varepsilon}=0, \\
\zeta_{\varepsilon} & \in \operatorname{im}\left(\mathcal{D}_{\left(u_{0}, A_{0}\right)}^{\varepsilon}\right)^{*} .
\end{aligned}
$$

(b) If $\left(u_{0}, A_{0}\right) \in \widetilde{\mathcal{M}}_{B, \Sigma}^{0}\left(c_{0}\right)$ and $(u, A)=\left(\exp _{u_{0}}(\xi), A_{0}+\alpha\right) \in \widetilde{\mathcal{M}}_{B, \Sigma}^{\varepsilon}$ where $\zeta=(\xi, \alpha) \in T_{\left(u_{0}, A_{0}\right)} \mathcal{B}$ satisfies (3.1) and

$$
\|\zeta\|_{1, p, \varepsilon ;\left(u_{0}, A_{0}\right)} \leq \delta \varepsilon^{2 / p+1 / 2}
$$

then $(u, A)=\widetilde{\mathcal{T}}^{\varepsilon}\left(u_{0}, A_{0}\right)$.

The map $\widetilde{\mathcal{T}}^{\varepsilon}$ of Theorem 1.1 descends to a map between the quotient spaces which we denote by

$$
\mathcal{T}^{\varepsilon}: \mathcal{M}_{B, \Sigma}^{0}\left(c_{0}\right) \longrightarrow \mathcal{M}_{B, \Sigma}^{\varepsilon}
$$

Assertion (a) is proved by a Newton-type iteration (see Section 6). It requires linear and quadratic estimates for the $\varepsilon$-dependent norms with constants that are independent of $\varepsilon$. These estimates are proved in Sections 4 and 5. Assertion (b) is a strengthened form of the corresponding uniqueness statement. Here the neighbourhood in which uniqueness holds is larger than in the existence result (namely it is of radius $c \varepsilon^{2 / p+1 / 2}$ instead of $c \varepsilon^{2}$ ). The uniqueness statement shows that the maps $\mathcal{T}^{\varepsilon}$ are independent of $c_{0}$ in the sense that two such maps corresponding to different values of $c_{0}$ (but the same value of $\varepsilon$ ) agree on the intersection of their domains. The next theorem shows that $\mathcal{T}^{\varepsilon}$ is locally surjective.

Theorem 3.2. Assume $(H 1)$ and $(H 4)$ and let $\bar{B} \in H_{2}(\bar{M} ; \mathbb{Z})$ be a nontorsion homology class. Then, for every $c_{0}>0$ and every $p>2$, there exist positive constants $\varepsilon_{0}$ and $\delta$ such that the following holds for every $\varepsilon \in\left(0, \varepsilon_{0}\right]$. If

$$
\left(\bar{u}_{0}, \bar{A}_{0}\right) \in \widetilde{\mathcal{M}}_{B, \Sigma}^{0}\left(c_{0}-1\right), \quad(u, A)=\left(\exp _{\bar{u}_{0}}(\bar{\xi}), \bar{A}_{0}+\bar{\alpha}\right) \in \widetilde{\mathcal{M}}_{B, \Sigma}^{\varepsilon},
$$

where $\bar{\zeta}=(\bar{\xi}, \bar{\alpha}) \in T_{\left(\bar{u}_{0}, \bar{A}_{0}\right)} \mathcal{B}$ satisfies

$$
\|\bar{\zeta}\|_{1, p, \varepsilon ;\left(\bar{u}_{0}, \bar{A}_{0}\right)} \leq \delta \varepsilon^{2 / p+1 / 2}
$$

then $(u, A) \in \widetilde{\mathcal{T}}^{\varepsilon}\left(\widetilde{\mathcal{M}}_{B, \Sigma}^{0}\left(c_{0}\right)\right)$.

This result is restated more precisely in Theorem 8.1. There it is proved that $g^{*}(u, A)=\widetilde{\mathcal{T}}^{\varepsilon}\left(u_{0}, A_{0}\right)$ for some gauge transformation $g$ and some pair $\left(u_{0}, A_{0}\right)$ in the image of the map $\mathcal{F}^{0}$ of Theorem 2.1. Moreover, it is shown that the distances of $g$ to $\mathbb{1}$ (in the $(2, p, \varepsilon)$-norm) and of $\left(u_{0}, A_{0}\right)$ to $\left(\bar{u}_{0}, \bar{A}_{0}\right)$ (in any norm) are controlled by the $(1, p, \varepsilon)$-norm of $\bar{\zeta}$. 
Theorem 3.2 strengthens the local uniqueness result of assertion (b) is Theorem 3.1 in that condition (3.1) is no longer required. The proof relies on an $\varepsilon$-dependent local slice theorem (Section 7 ) and on the construction of a tubular neighbourhood of the moduli space $\mathcal{M}_{B, \Sigma}^{0}\left(c_{0}\right)$ in which the normal bundle is the intersection of the $\varepsilon$-dependent local slice with the image of the adjoint operator $\mathcal{D}^{\varepsilon *}$ (Section 8).

The next theorem strengthens the local surjectivity result of Theorem 3.2. It does not require the solution $(u, A)$ of equations (1.7) to be close to any solution of equation (1.8). However, it only applies to solutions that satisfy a uniform $L^{\infty}$-bound on $d_{A} u$ and for which $u$ takes values in the compact set

$$
M^{C}:=\left\{x \in M|| \mu(x)|\leq C,| \eta|\leq C| L_{x} \eta \mid \forall \eta \in \mathfrak{g}\right\} .
$$

Theorem 3.3. Assume $(H 1)$ and $(H 4)$ and let $\bar{B} \in H_{2}(\bar{M} ; \mathbb{Z})$ be a nontorsion homology class. Then, for every $C>0$, there exist positive constants $\varepsilon_{0}$ and $c_{1}$ such that the following holds for every $\varepsilon \in\left(0, \varepsilon_{0}\right]$. If $(u, A) \in \widetilde{\mathcal{M}}_{B, \Sigma}^{\varepsilon}$ such that

$$
\left\|d_{A} u\right\|_{L^{\infty}} \leq C, \quad u(P) \subset M^{C},
$$

then $(u, A) \in \widetilde{\mathcal{T}}^{\varepsilon}\left(\widetilde{\mathcal{M}}_{B, \Sigma}^{0}\left(c_{1}\right)\right)$.

Under hypotheses (H1) and (H2), the moduli space $\mathcal{M}_{B, \Sigma}^{\varepsilon}$ is compact [2]. In this case all solutions of equations (1.7) satisfy $\left\|d_{A} u\right\|_{L^{\infty}}+\|\mu(u)\|_{L^{\infty}} \leq$ $C_{\varepsilon}$ for some $\varepsilon$-dependent constant $C_{\varepsilon}$. However, this does not guarantee surjectivity because, on the one hand, the constant $C_{\varepsilon}$ may diverge to infinity as $\varepsilon$ tends to zero and, on the other hand, the solutions of equations (1.7) may not all satisfy the second condition in the definiton of $M^{C}$, namely that the image of $u$ belongs to the set of regular points of $\mu$. There may be sequences $\left(\varepsilon_{i}, u_{i}, A_{i}\right)$ of solutions of equations (1.7) with $\varepsilon_{i} \rightarrow 0$ such that either $u_{i}(P)$ intersects the set of singular points of $\mu$ or $d_{A_{i}} u_{i}$ does not stay bounded, and then bubbling occurs in the small $\varepsilon$ limit. Under the hypotheses of Theorem 1.1 we shall prove that such bubbling cannot occur and establish a bijection between suitable zero-dimensional moduli spaces.

\section{Linear estimates}

The estimates in this section follow the ones in [6, Section 4]. In adapting the proofs to the present context, we encounter additional zeroth-order terms. These arise from the Levi-Civita connection and the almost complex structure on $M$; they are not present in [6] where $M$ is replaced by the space of connections over a Riemann surface and the almost complex structure by the Hodge $*$-operator. We extend the results of $[\mathbf{6}]$ by including estimates for the second derivatives. Moreover, in the present case it is crucial that the constants depend continuously on the pair $(u, A)$. In $[\mathbf{6}]$, the moduli 
space is a finite set and so the question of continuous dependence does not arise.

For $u \in C_{\mathrm{G}}^{\infty}(P, M)$, we introduce the spaces

$$
\begin{aligned}
& \mathcal{X}_{u}:=\Omega^{0}\left(\Sigma, u^{*} T M / \mathrm{G}\right) \times \Omega^{1}\left(\Sigma, \mathfrak{g}_{P}\right), \\
& \mathcal{X}_{u}^{\prime}:=\Omega^{0,1}\left(\Sigma, u^{*} T M / \mathrm{G}\right) \times \Omega^{0}\left(\Sigma, \mathfrak{g}_{P}\right) \times \Omega^{0}\left(\Sigma, \mathfrak{g}_{P}\right) .
\end{aligned}
$$

Thus $\mathcal{X}_{u}=T_{(u, A)} \mathcal{B}$ and $\mathcal{X}_{u}^{\prime}=\mathcal{E}_{(u, A)} \times \Omega^{0}\left(\Sigma, \mathfrak{g}_{P}\right)$ for every $A \in \mathcal{A}(P)$. If the map $u$ is understood from the context, then we shall omit the subscript $u$. It is convenient to introduce the norms

$$
\begin{aligned}
\|\xi\|_{1, p, \varepsilon ;(u, A)} & :=\|\xi\|_{L^{p}}+\varepsilon\left\|\tilde{\nabla}_{A} \xi\right\|_{L^{p}}, \\
\|\xi\|_{2, p, \varepsilon ;(u, A)} & :=\|\xi\|_{L^{p}}+\varepsilon\left\|\tilde{\nabla}_{A} \xi\right\|_{L^{p}}+\varepsilon^{2}\left\|\tilde{\nabla}_{A}^{*} \tilde{\nabla}_{A} \xi\right\|_{L^{p}}, \\
\|\alpha\|_{1, p, \varepsilon ; A} & :=\|\alpha\|_{L^{p}}+\varepsilon\left\|d_{A} \alpha\right\|_{L^{p}}+\varepsilon\left\|d_{A}^{*} \alpha\right\|_{L^{p}}, \\
\|\alpha\|_{2, p, \varepsilon ; A} & :=\|\alpha\|_{L^{p}}+\varepsilon\left\|d_{A} \alpha\right\|_{L^{p}}+\varepsilon\left\|d_{A}^{*} \alpha\right\|_{L^{p}}+\varepsilon^{2}\left\|d_{A}^{*} d_{A} \alpha+d_{A} d_{A}^{*} \alpha\right\|_{L^{p}},
\end{aligned}
$$

for $\xi \in \Omega^{0, k}\left(\Sigma, u^{*} T M / \mathrm{G}\right), \alpha \in \Omega^{k}\left(\Sigma, \mathfrak{g}_{P}\right), k=0,1$, and $1 \leq p \leq \infty$. Here $\tilde{\nabla}_{A}$ denotes the Hermitian connection on $u^{*} T M / \mathrm{G}$ defined by equation (B.2). For $\ell=0,1,2,1 \leq p \leq \infty$, and $\zeta=(\xi, \alpha) \in \mathcal{X}_{u}, \zeta^{\prime}=\left(\xi^{\prime}, \varphi^{\prime}, \psi^{\prime}\right) \in \mathcal{X}_{u}^{\prime}$, we consider the norms

$$
\begin{aligned}
\|\zeta\|_{\ell, p, \varepsilon ;(u, A)} & :=\|\xi\|_{\ell, p, \varepsilon ;(u, A)}+\varepsilon\|\alpha\|_{\ell, p, \varepsilon ;(u, A)}, \\
\left\|\zeta^{\prime}\right\|_{\ell, p, \varepsilon ;(u, A)} & :=\left\|\xi^{\prime}\right\|_{\ell, p, \varepsilon ;(u, A)}+\varepsilon\left\|\varphi^{\prime}\right\|_{\ell, p, \varepsilon ;(u, A)}+\varepsilon\left\|\psi^{\prime}\right\|_{\ell, p, \varepsilon ;(u, A)},
\end{aligned}
$$

where $\|\xi\|_{0, p, \varepsilon ;(u, A)}:=\|\xi\|_{L^{p}}$. These norms are gauge-invariant. If the pair $(u, A)$ is understood from the context, we shall drop it to simplify the notation. In particular, we abbreviate $\|\zeta\|_{\infty, \varepsilon}:=\|\zeta\|_{0, \infty, \varepsilon ;(u, A)}$.

The augmented vertical differential of $\mathcal{F}^{\varepsilon}$ at a zero $(u, A) \in \mathcal{B}$ is the operator $\mathcal{D}^{\varepsilon}=\mathcal{D}_{(u, A)}^{\varepsilon}: \mathcal{X}_{u} \rightarrow \mathcal{X}_{u}^{\prime}$ given by

$$
\mathcal{D}^{\varepsilon}\left(\begin{array}{c}
\xi \\
\alpha
\end{array}\right)=\left(\begin{array}{c}
D \xi+\left(L_{u} \alpha\right)^{0,1} \\
\varepsilon^{-2} L_{u}^{*} \xi-d_{A}^{*} \alpha \\
\varepsilon^{-2} d \mu(u) \xi+* d_{A} \alpha
\end{array}\right),
$$

where $D=D_{(u, A)}: \Omega^{0}\left(\Sigma, u^{*} T M / \mathrm{G}\right) \rightarrow \Omega^{0,1}\left(\Sigma, u^{*} T M / \mathrm{G}\right)$ is the CauchyRiemann operator defined by equation (B.3). The second coordinate in the definition of $\mathcal{D}^{\varepsilon}$ corresponds to the local slice condition for the $\mathcal{G}$-action. For the definition of the adjoint operator, it is convenient to use the $\varepsilon$-dependent inner products associated to the $(0,2, \varepsilon)$-norms. In addition, we use twice the standard inner product on the space $\Omega^{0,1}\left(\Sigma, u^{*} T M / \mathrm{G}\right)$. Then the adjoint of $\mathcal{D}^{\varepsilon}$ is given by

$$
\mathcal{D}^{\varepsilon *}\left(\begin{array}{c}
\xi^{\prime} \\
\varphi^{\prime} \\
\psi^{\prime}
\end{array}\right)=\left(\begin{array}{c}
2 D^{*} \xi^{\prime}+L_{u} \varphi^{\prime}+J L_{u} \psi^{\prime} \\
2 \varepsilon^{-2} L_{u}^{*} \xi^{\prime}-d_{A} \varphi^{\prime}-* d_{A} \psi^{\prime}
\end{array}\right)
$$


Remark 4.1 (Local coordinates). Let $v: U \rightarrow \Sigma$ be a holomorphic coordinate chart defined on an open set $U \subset \mathbb{C}$ and let $\tilde{v}: U \rightarrow P$ be a lift of $v$. In this trivialization the map $u$, the connection $A$, the vector field $\xi$ along $u$, and the 1-form $\alpha$ are represented by

$$
\begin{array}{ll}
u^{\mathrm{loc}}:=u \circ \tilde{v}, & A^{\mathrm{loc}}:=\tilde{v}^{*} A=\Phi d s+\Psi d t, \\
\xi^{\mathrm{loc}}:=\xi \circ \tilde{v}, & \alpha^{\mathrm{loc}}:=\tilde{v}^{*} \alpha=\varphi d s+\psi d t,
\end{array}
$$

where $\Phi, \Psi, \varphi$, and $\psi$ are Lie-algebra-valued functions on $U$. The volume form on $U$ is given by $\lambda^{2} d s \wedge d t:=v^{*} \mathrm{dvol}_{\Sigma}$ for some function $\lambda: U \rightarrow(0, \infty)$ and the metric has the form $\lambda^{2}\left(d s^{2}+d t^{2}\right)$. From now on we shall drop the superscript "loc" and introduce the notation

$$
\begin{aligned}
v_{s} & :=\partial_{s} u+X_{\Phi}(u), & & v_{t}:=\partial_{t} u+X_{\Psi}(u), \\
\nabla_{A, s} \xi & :=\nabla_{s} \xi+\nabla_{\xi} X_{\Phi}(u), & & \nabla_{A, t} \xi:=\nabla_{t} \xi+\nabla_{\xi} X_{\Psi}(u), \\
\tilde{\nabla}_{A, s} \xi & :=\nabla_{A, s} \xi-\frac{1}{2} J\left(\nabla_{v_{s}} J+\partial_{s} J\right) \xi, & & \tilde{\nabla}_{A, t} \xi:=\nabla_{A, t} \xi-\frac{1}{2} J\left(\nabla_{v_{t}} J+\partial_{t} J\right) \xi, \\
\nabla_{A, s} \eta & :=\partial_{s} \eta+[\Phi, \eta], & & \nabla_{A, t} \eta:=\partial_{t} \eta+[\Psi, \eta],
\end{aligned}
$$

for $\eta: U \rightarrow \mathfrak{g}$ and a vector field $\xi: U \rightarrow u^{*} T M$ along $u$. Then

$$
\begin{array}{ll}
d_{A} u=v_{s} d s+v_{t} d t, & \nabla_{A} \xi=\nabla_{A, s} \xi d s+\nabla_{A, t} \xi d t, \\
* F_{A}=\lambda^{-2}\left(\partial_{s} \Psi-\partial_{t} \Phi+[\Phi, \Psi]\right), & * d_{A} \alpha=\lambda^{-2}\left(\nabla_{A, s} \psi-\nabla_{A, t} \varphi\right), \\
& d_{A}^{*} \alpha=-\lambda^{-2}\left(\nabla_{A, s} \varphi+\nabla_{A, t} \psi\right) .
\end{array}
$$

In local coordinates a $(0,1)$-form on $\Sigma$ with values in $u^{*} T M / \mathrm{G}$ has the form $1 / 2\left(\xi^{\prime} d s-J \xi^{\prime} d t\right)$, where $\xi^{\prime}(s, t) \in T_{u(s, t)} M$. In particular,

$$
\begin{aligned}
\bar{\partial}_{J, A}(u) & =\frac{1}{2}\left(v_{s}+J v_{t}\right) d s+\frac{1}{2}\left(v_{t}-J v_{s}\right) d t \\
\left(\nabla_{A} \xi\right)^{0,1} & =\frac{1}{2}\left(\nabla_{A, s} \xi+J \nabla_{A, t} \xi\right) d s+\frac{1}{2}\left(\nabla_{A, t} \xi-J \nabla_{A, s} \xi\right) d t
\end{aligned}
$$

We represent a $(0,1)$-form by twice the coefficient of $d s$. Then

$$
D \xi=\tilde{\nabla}_{A, s} \xi+J \tilde{\nabla}_{A, t} \xi+\frac{1}{4} N\left(\xi, v_{s}-J v_{t}\right)+\frac{1}{2}\left(J \partial_{s} J-\partial_{t} J\right) \xi,
$$

where $N\left(\xi_{1}, \xi_{2}\right)=2 J\left(\left(\nabla_{\xi_{2}} J\right) \xi_{1}-\left(\nabla_{\xi_{1}} J\right) \xi_{2}\right)$ denotes the Nijenhuis tensor, and $2 D^{*} \xi^{\prime}=\frac{1}{\lambda^{2}}\left(-\tilde{\nabla}_{A, s} \xi^{\prime}+J \tilde{\nabla}_{A, t} \xi^{\prime}-\frac{1}{2} J\left(\nabla_{\xi^{\prime}} J\right)\left(v_{s}-J v_{t}\right)+\frac{1}{2}\left(J \partial_{s} J-\partial_{t} J\right) \xi\right)$.

The Weitzenböck formula has the form $D^{*} D \xi=1 / 2 \tilde{\nabla}_{A}{ }^{*} \tilde{\nabla}_{A} \xi+$ l.o.t. In the Kähler case, we have $\nabla_{A}=\tilde{\nabla}_{A}, \nabla J=0$, and $\partial_{s} J=\partial_{t} J=0$. Hence in this 
case

$$
\begin{aligned}
D^{*} D \xi= & -\frac{1}{2 \lambda^{2}}\left(\nabla_{A, s} \nabla_{A, s} \xi+\nabla_{A, t} \nabla_{A, t} \xi\right) \\
& -\frac{1}{2 \lambda^{2}} J R\left(v_{s}, v_{t}\right) \xi-\frac{1}{2 \lambda^{2}} J \nabla_{\xi} X_{\partial_{s} \Psi-\partial_{t} \Phi+[\Phi, \Psi]}(u) .
\end{aligned}
$$

In local coordinates, the operators $\mathcal{D}^{\varepsilon}$ and $\mathcal{D}^{\varepsilon *}$ have the form

$$
\begin{gathered}
\mathcal{D}^{\varepsilon} \zeta=\left(\begin{array}{c}
D \xi+L_{u} \varphi+J L_{u} \psi \\
\lambda^{-2}\left(\nabla_{A, s} \varphi+\nabla_{A, t} \psi\right)+\varepsilon^{-2} L_{u}^{*} \xi \\
\lambda^{-2}\left(\nabla_{A, s} \psi-\nabla_{A, t} \varphi\right)+\varepsilon^{-2} d \mu(u) \xi
\end{array}\right), \\
\mathcal{D}^{\varepsilon *} \zeta^{\prime}=\left(\begin{array}{c}
2 D^{*}+L_{u} \varphi^{\prime}+J L_{u} \psi^{\prime} \\
-\nabla_{A, s} \varphi^{\prime}+\nabla_{A, t} \psi^{\prime}+\varepsilon^{-2} L_{u}^{*} \xi^{\prime} \\
-\nabla_{A, s} \psi^{\prime}-\nabla_{A, t} \varphi^{\prime}+\varepsilon^{-2} d \mu(u) \xi^{\prime}
\end{array}\right) .
\end{gathered}
$$

Proposition 4.2. For every $p \geq 2$ and every $c_{0}>0$, there exist positive constants $\varepsilon_{0}$ and $c$ such that

$$
\begin{aligned}
\|\zeta\|_{1, p, \varepsilon ;(u, A)} & \leq c\left(\varepsilon\left\|\mathcal{D}^{\varepsilon} \zeta\right\|_{0, p, \varepsilon}+\left\|\pi_{u} \xi\right\|_{L^{p}}\right), \\
\left\|\zeta-\pi_{u} \zeta\right\|_{1, p, \varepsilon ;(u, A)} & \leq c \varepsilon\left(\left\|\mathcal{D}^{\varepsilon} \zeta\right\|_{0, p, \varepsilon}+\left\|\pi_{u} \xi\right\|_{L^{p}}\right), \\
\left\|\zeta^{\prime}\right\|_{1, p, \varepsilon ;(u, A)} & \leq c\left(\varepsilon\left\|\mathcal{D}^{\varepsilon *} \zeta^{\prime}\right\|_{0, p, \varepsilon}+\left\|\pi_{u} \xi^{\prime}\right\|_{L^{p}}\right), \\
\left\|\zeta^{\prime}-\pi_{u} \zeta^{\prime}\right\|_{1, p, \varepsilon ;(u, A)} & \leq c \varepsilon\left(\left\|\mathcal{D}^{\varepsilon *} \zeta^{\prime}\right\|_{0, p, \varepsilon}+\left\|\pi_{u} \xi^{\prime}\right\|_{L^{p}}\right),
\end{aligned}
$$

for all $(u, A) \in \widetilde{\mathcal{M}}_{B, \Sigma}^{0}\left(c_{0}\right), \zeta=(\xi, \alpha) \in \mathcal{X}_{u}, \zeta^{\prime}=\left(\xi^{\prime}, \varphi^{\prime}, \psi^{\prime}\right) \in \mathcal{X}_{u}^{\prime}$, and $\varepsilon \in\left(0, \varepsilon_{0}\right]$. Here we abbreviate $\mathcal{D}^{\varepsilon}:=\mathcal{D}_{(u, A)}^{\varepsilon}$ and $\pi_{u} \zeta:=\left(\pi_{u} \xi, 0\right)$ and $\pi_{u} \zeta^{\prime}:=$ $\left(\pi_{u} \xi^{\prime}, 0,0\right)$, where $\pi_{u}$ is defined by (2.1).

In this paper, we prove Proposition 4.2 only in the case $p=2$. The proof for $p>2$ is similar to the proof of an analogous result in $[\mathbf{1 6}]$.

\section{Lemma 4.3.}

(i) If $\bar{\partial}_{J, A}(u)=0$ then

$$
\mathcal{D}^{\varepsilon} \mathcal{D}^{\varepsilon *} \zeta^{\prime}=\left(\begin{array}{c}
2 D D^{*} \xi^{\prime}+2 \varepsilon^{-2}\left(L_{u} L_{u}^{*} \xi^{\prime}\right)^{0,1}+(D J-J D) L_{u} \psi^{\prime} \\
\Delta_{\varepsilon} \varphi^{\prime}+\left[* F_{A}+\varepsilon^{-2} \mu(u), \psi^{\prime}\right] \\
\Delta_{\varepsilon} \psi^{\prime}-\left[* F_{A}+\varepsilon^{-2} \mu(u), \varphi^{\prime}\right]+2 \varepsilon^{-2} L_{u}^{*}(D J-J D)^{*} \xi^{\prime}
\end{array}\right)
$$

for $\zeta^{\prime}:=\left(\xi^{\prime}, \varphi^{\prime}, \psi^{\prime}\right) \in \mathcal{X}_{u}^{\prime}$, where $\Delta_{\varepsilon}:=d_{A}^{*} d_{A}+\varepsilon^{-2} L_{u}^{*} L_{u}$.

(ii) If $\bar{\partial}_{J, A}(u)=0$ and $\mu(u)=0$, then

$$
\mathcal{D}^{\varepsilon *} \mathcal{D}^{\varepsilon} \zeta=\left(\begin{array}{c}
2 D^{*} D \xi+\varepsilon^{-2} L_{u} L_{u}^{*} \xi+\varepsilon^{-2} J L_{u} L_{u}^{*} J^{*} \xi+Q^{*} \alpha \\
d_{A}^{*} d_{A} \alpha+d_{A} d_{A}^{*} \alpha+\varepsilon^{-2} L_{u}^{*} L_{u} \alpha+\varepsilon^{-2} Q \xi
\end{array}\right),
$$


for $\zeta=(\xi, \alpha) \in \mathcal{X}_{u}$, where $Q: \Omega^{0}\left(\Sigma, u^{*} T M / \mathrm{G}\right) \rightarrow \Omega^{1}\left(\Sigma, \mathfrak{g}_{P}\right)$ denotes the zeroth-order operator

$$
\begin{aligned}
Q \xi & :=2 L_{u}^{*} D \xi-d_{A} L_{u}^{*} \xi-* d_{A} d \mu(u) \xi \\
& =\rho\left(\xi, d_{A} u\right)-* \rho\left(J \xi, d_{A} u\right)+* L_{u}^{*} \dot{J} \xi+\frac{1}{2} L_{u}^{*} N\left(\xi, \partial_{J, A}(u)\right) .
\end{aligned}
$$

Proof. We shall repeatedly use the identities

$$
\begin{aligned}
d_{A}^{*} \alpha & =-* d_{A} * \alpha, \quad * d_{A} d_{A} \varphi=\left[* F_{A}, \varphi\right], \\
L_{u}^{*} & =d \mu(u) J, \quad d \mu(u) L_{u} \varphi=-[\mu(u), \varphi],
\end{aligned}
$$

for $\alpha \in \Omega^{1}\left(\Sigma, \mathfrak{g}_{P}\right)$ and $\varphi \in \Omega^{0}\left(\Sigma, \mathfrak{g}_{P}\right)$. To prove (i), note that the triple $\left(\tilde{\xi}^{\prime}, \tilde{\varphi}^{\prime}, \tilde{\psi}^{\prime}\right):=\mathcal{D}^{\varepsilon} \mathcal{D}^{\varepsilon *}\left(\xi^{\prime}, \varphi^{\prime}, \psi^{\prime}\right)$ is given by

$$
\begin{aligned}
\tilde{\xi}^{\prime}= & D\left(2 D^{*} \xi^{\prime}+L_{u} \varphi^{\prime}+J L_{u} \psi^{\prime}\right)+\left(L_{u}\left(2 \varepsilon^{-2} L_{u}^{*} \xi^{\prime}-d_{A} \varphi^{\prime}-* d_{A} \psi^{\prime}\right)\right)^{0,1} \\
= & 2 D D^{*} \xi^{\prime}+2 \varepsilon^{-2}\left(L_{u} L_{u}^{*} \xi^{\prime}\right)^{0,1}+D L_{u} \varphi^{\prime}-\left(L_{u} d_{A} \varphi^{\prime}\right)^{0,1} \\
& +D J L_{u} \psi^{\prime}-\left(L_{u} * d_{A} \psi^{\prime}\right)^{0,1}, \\
\tilde{\varphi}^{\prime}= & \varepsilon^{-2} L_{u}^{*}\left(2 D^{*} \xi^{\prime}+L_{u} \varphi+J L_{u} \psi\right)-d_{A}^{*}\left(2 \varepsilon^{-2} L_{u}^{*} \xi^{\prime}-d_{A} \varphi-* d_{A} \psi\right) \\
= & d_{A}^{*} d_{A} \varphi^{\prime}+\varepsilon^{-2} L_{u}^{*} L_{u} \varphi^{\prime}+2 \varepsilon^{-2}\left(D L_{u}-L_{u} d_{A}\right)^{*} \xi^{\prime}+\left[* F_{A}+\varepsilon^{-2} \mu(u), \psi^{\prime}\right], \\
\tilde{\psi}^{\prime}= & \varepsilon^{-2} d \mu(u)\left(2 D^{*} \xi^{\prime}+L_{u} \varphi^{\prime}+J L_{u} \psi^{\prime}\right)+* d_{A}\left(2 \varepsilon^{-2} L_{u}^{*} \xi^{\prime}-d_{A} \varphi^{\prime}-* d_{A} \psi^{\prime}\right) \\
= & d_{A}^{*} d_{A} \psi^{\prime}+\varepsilon^{-2} L_{u}^{*} L_{u} \psi^{\prime}-\left[* F_{A}+\varepsilon^{-2} \mu(u), \varphi^{\prime}\right] \\
& +2 \varepsilon^{-2}\left(L_{u}^{*} J^{*} D^{*} \xi^{\prime}+d_{A}^{*} * L_{u}^{*} \xi^{\prime}\right) .
\end{aligned}
$$

The assertion now follows from the fact that

$$
J\left(L_{u} \alpha\right)^{0,1}=\left(L_{u} * \alpha\right)^{0,1}, \quad L_{u}^{*} J^{*} \xi^{\prime}=-* L_{u}^{*} \xi^{\prime},
$$

for $\alpha \in \Omega^{1}\left(\Sigma, \mathfrak{g}_{P}\right)$ and $\xi^{\prime} \in \Omega^{0,1}\left(\Sigma, u^{*} T M / \mathrm{G}\right)$, and

$$
\bar{\partial}_{J, A}(u)=0 \Longrightarrow D L_{u} \varphi=\left(L_{u} d_{A} \varphi\right)^{0,1}
$$

for $\varphi \in \Omega^{0}\left(\Sigma, \mathfrak{g}_{P}\right)$. The first part in equations (4.2) follows from the fact that $* \alpha=-\alpha \circ J_{\Sigma}$ for every 1-form $\alpha$ on $\Sigma$ (with values in any vector bundle). The second part equations (4.2) follows from the first by duality. Equation (4.3) follows from the fact that the section $(u, A) \mapsto \bar{\partial}_{J, A}(u)$ of the vector bundle over $\mathcal{B}$ with fibres $\Omega^{0,1}\left(\Sigma, u^{*} T M / \mathrm{G}\right)$ is $\mathcal{G}(P)$-equivariant.

To prove (ii), note that the pair

$$
(\tilde{\xi}, \tilde{\alpha}):=\mathcal{D}^{\varepsilon *} \mathcal{D}^{\varepsilon}(\xi, \alpha)
$$

is given by

$$
\begin{aligned}
\tilde{\xi} & =2 D^{*}\left(D \xi+\left(L_{u} \alpha\right)^{0,1}\right)+L_{u}\left(\varepsilon^{-2} L_{u}^{*} \xi-d_{A}^{*} \alpha\right)+J L_{u}\left(\varepsilon^{-2} d \mu(u) \xi+* d_{A} \alpha\right) \\
& =2 D^{*} D \xi+2 \varepsilon^{-2}\left(L_{u} L_{u}^{*} \xi^{\prime}\right)^{0,1}+\left(2 D^{*}\left(L_{u} \alpha\right)^{0,1}-L_{u} d_{A}^{*} \alpha+J L_{u} * d_{A} \alpha\right), \\
\tilde{\alpha} & =2 \varepsilon^{-2} L_{u}^{*}\left(D \xi+\left(L_{u} \alpha\right)^{0,1}\right)-d_{A}\left(\varepsilon^{-2} L_{u}^{*} \xi-d_{A}^{*} \alpha\right)-* d_{A}\left(\varepsilon^{-2} d \mu(u) \xi+* d_{A} \alpha\right) \\
& =d_{A}^{*} d_{A} \alpha+d_{A} d_{A}^{*} \alpha+\varepsilon^{-2} L_{u}^{*} L_{u} \alpha+\varepsilon^{-2}\left(2 L_{u}^{*} D \xi-d_{A} L_{u}^{*} \xi-* d_{A} d \mu(u) \xi\right) .
\end{aligned}
$$


Here we have used the fact that $\mu(u)=0$ and hence $2 L_{u}^{*}\left(L_{u} \alpha\right)^{0,1}=L_{u}^{*} L_{u} \alpha$. The formula for the operator $Q:=2 L_{u}^{*} D-d_{A} L_{u}^{*}-* d_{A} d \mu(u)$ follows by computing in local coordinates.

Proof of Proposition 4.2 for $p=2$. Let

$$
\zeta^{\prime}:=\left(\xi^{\prime}, \varphi^{\prime}, \psi^{\prime}\right):=\mathcal{D}^{\varepsilon} \zeta .
$$

Then, by Lemma 4.3, the formula

$$
\mathcal{D}^{\varepsilon *} \mathcal{D}^{\varepsilon} \zeta=\mathcal{D}^{\varepsilon *} \zeta^{\prime}
$$

is equivalent to

$$
\begin{aligned}
2 D^{*} D \xi+\varepsilon^{-2} L_{u} L_{u}^{*} \xi+\varepsilon^{-2} J L_{u} L_{u}^{*} J^{*} \xi+Q^{*} \alpha & =2 D^{*} \xi^{\prime}+L_{u} \varphi^{\prime}+J L_{u} \psi^{\prime}, \\
d_{A}^{*} d_{A} \alpha+d_{A} d_{A}^{*} \alpha+\varepsilon^{-2} L_{u}^{*} L_{u} \alpha+\varepsilon^{-2} Q \xi & =2 \varepsilon^{-2} L_{u}^{*} \xi^{\prime}-d_{A} \varphi^{\prime}-* d_{A} \psi^{\prime} .
\end{aligned}
$$

Take the $L^{2}$-inner product of the first equation with $\xi$ and of the second equation with $\varepsilon^{2} \alpha$. The sum of the resulting identities gives

$$
\begin{aligned}
\varepsilon^{-2} \| & L_{u}^{*} \xi\left\|^{2}+\varepsilon^{-2}\right\| L_{u}^{*} J \xi\left\|^{2}+2\right\| D \xi\left\|^{2}+\right\| L_{u} \alpha\left\|^{2}+\varepsilon^{2}\right\| d_{A} \alpha\left\|^{2}+\varepsilon^{2}\right\| d_{A}^{*} \alpha \|^{2} \\
= & 2\left\langle\xi^{\prime}, D \xi\right\rangle+2\left\langle\xi^{\prime}, L_{u} \alpha\right\rangle-2\langle\alpha, Q \xi\rangle+\left\langle\varphi^{\prime}, L_{u}^{*} \xi\right\rangle-\left\langle\psi^{\prime}, L_{u}^{*} J \xi\right\rangle \\
& -\varepsilon^{2}\left\langle\varphi^{\prime}, d_{A}^{*} \alpha\right\rangle+\varepsilon^{2}\left\langle\psi^{\prime}, * d_{A} \alpha\right\rangle \\
\leq & 3\left\|\xi^{\prime}\right\|^{2}+\|D \xi\|^{2}+2^{-1}\left\|L_{u} \alpha\right\|^{2}+\delta\|\alpha\|^{2}+\delta^{-1}\|Q \xi\|^{2}+\varepsilon^{2}\left\|\varphi^{\prime}\right\|^{2} \\
& +\varepsilon^{2}\left\|\psi^{\prime}\right\|^{2}+2^{-1} \varepsilon^{-2}\left\|L_{u}^{*} \xi\right\|^{2}+2^{-1} \varepsilon^{-2}\left\|L_{u}^{*} J \xi\right\|^{2}+2^{-1} \varepsilon^{2}\left\|d_{A}^{*} \alpha\right\|^{2} \\
& +2^{-1} \varepsilon^{2}\left\|d_{A} \alpha\right\|^{2} .
\end{aligned}
$$

Here all norms are $L^{2}$-norms and all inner products are $L^{2}$-inner products. Choose $\delta>0$ so small that $\delta\|\alpha\|^{2} \leq 4^{-1}\left\|L_{u} \alpha\right\|^{2}$ for all $\alpha$. Then

$$
\begin{aligned}
& \varepsilon^{-2}\left\|L_{u}^{*} \xi\right\|^{2}+\varepsilon^{-2}\left\|L_{u}^{*} J \xi\right\|^{2}+\|D \xi\|^{2}+\left\|L_{u} \alpha\right\|^{2}+\varepsilon^{2}\left\|d_{A} \alpha\right\|^{2}+\varepsilon^{2}\left\|d_{A}^{*} \alpha\right\|^{2} \\
& \quad \leq 12\left\|\xi^{\prime}\right\|^{2}+4 \varepsilon^{2}\left\|\varphi^{\prime}\right\|^{2}+4 \varepsilon^{2}\left\|\psi^{\prime}\right\|^{2}+4 \delta^{-1}\|Q\|_{L^{\infty}}^{2}\|\xi\|^{2} \\
& \quad \leq 12\left\|\mathcal{D}^{\varepsilon}(\xi, \alpha)\right\|_{0,2, \varepsilon}^{2}+4 \delta^{-1}\|Q\|_{L^{\infty}}^{2}\|\xi\|_{L^{2}}^{2} .
\end{aligned}
$$

Now the required estimates follow from the inequalities

$$
\begin{aligned}
\left\|\tilde{\nabla}_{A} \pi_{u} \xi\right\|_{L^{2}} & \leq c\left(\left\|\tilde{\nabla}_{A} \xi\right\|_{L^{2}}+\|\xi\|_{L^{2}}\right), \\
\left\|\xi-\pi_{u} \xi\right\|_{L^{2}} & \leq c^{\prime}\left(\left\|L_{u}^{*} \xi\right\|_{L^{2}}+\left\|L_{u}^{*} J \xi\right\|_{L^{2}}\right), \\
\left\|\tilde{\nabla}_{A} \xi\right\|_{L^{2}} & \leq c^{\prime \prime}\left(\|D \xi\|_{L^{2}}+\|\xi\|_{L^{2}}\right) .
\end{aligned}
$$

The first inequality follows from equations (4.4) below. In the second inequality, the constant $c^{\prime}$ can be chosen as an upper bound for the norms of the linear maps $L_{x}\left(L_{x}^{*} L_{x}\right)^{-1}$ over all $x \in \mu^{-1}(0)$. The third inequality is the $L^{2}$-estimate for the Cauchy-Riemann operator and it follows from the Weitzenböck formula. The constant $c^{\prime \prime}$ is gauge-invariant and depends continuously on the pair $(u, A)$ with respect to the $C^{1}$-norm and hence can 
be chosen independent of $(u, A) \in \widetilde{\mathcal{M}}_{B, \Sigma}^{0}\left(c_{0}\right)$. This proves the proposition in the case $p=2$.

The next lemma expresses the Sobolev inequalities in terms of the $\varepsilon$-dependent norms.

Lemma 4.4. For every $p>2$ and every $c_{0}>0$, there exists a constant $c>0$ such that

$$
\|\zeta\|_{\infty, \varepsilon} \leq c \varepsilon^{-2 / p}\|\zeta\|_{1, p, \varepsilon ;(u, A)}, \quad\|\zeta\|_{1, \infty, \varepsilon} \leq c \varepsilon^{-2 / p}\|\zeta\|_{2, p, \varepsilon ;(u, A)}
$$

for all $(u, A) \in \widetilde{\mathcal{M}}_{B, \Sigma}^{0}\left(c_{0}\right), \zeta \in \mathcal{X}_{u}$, and $\varepsilon \in(0,1)$.

Proof. Multiply the metric on $\Sigma$ by $\varepsilon^{-2}$. Then the $W^{k, p}$-norm of $(\xi, \alpha)$ with respect to the rescaled metric is equal to $\varepsilon^{-2 / p}$ times the $(k, p, \varepsilon)$-norm of $\zeta$, and the $L^{\infty}$-norm with respect to the rescaled metric is equal to the $(\infty, \varepsilon)$ norm. Hence the estimates follow from the Sobolev embedding theorem for the rescaled metric. The constant is gauge-invariant and it depends continuously on $u$ (with respect to the $C^{1}$-norm) and $A$ (with respect to the $C^{0}$-norm). By Lemma 2.2, the estimate holds with a uniform constant $c$.

Lemma 4.5. For every $p \geq 2$ and every $c_{0}>0$, there exist positive constants $\varepsilon_{0}$ and $c$ such that

$$
\begin{aligned}
\left\|\pi_{u} \mathcal{D}^{\varepsilon} \zeta-\mathcal{D}^{0} \pi_{u} \zeta\right\|_{k, p, \varepsilon} & \leq c\left\|\xi-\pi_{u} \xi\right\|_{k, p, \varepsilon}, \\
\left\|\pi_{u} \mathcal{D}^{\varepsilon} \zeta-\mathcal{D}^{\varepsilon} \pi_{u} \zeta\right\|_{k, p, \varepsilon} & \leq c\|\xi\|_{k, p, \varepsilon}, \\
\left\|\pi_{u} \mathcal{D}^{\varepsilon *} \zeta^{\prime}-\mathcal{D}^{0^{*}} \pi_{u} \zeta^{\prime}\right\|_{k, p, \varepsilon} & \leq c\left\|\xi^{\prime}-\pi_{u} \xi^{\prime}\right\|_{k, p, \varepsilon}, \\
\left\|\pi_{u} \mathcal{D}^{\varepsilon *} \zeta^{\prime}-\mathcal{D}^{\varepsilon *} \pi_{u} \zeta^{\prime}\right\|_{k, p, \varepsilon} & \leq c\left\|\xi^{\prime}\right\|_{k, p, \varepsilon},
\end{aligned}
$$

for every $(u, A) \in \widetilde{\mathcal{M}}_{B, \Sigma}^{0}\left(c_{0}\right), \zeta=(\xi, \alpha) \in \mathcal{X}_{u}, \zeta^{\prime}=\left(\xi^{\prime}, \varphi^{\prime}, \psi^{\prime}\right) \in \mathcal{X}_{u}^{\prime}, \varepsilon \in$ $(0,1]$, and $k=0,1$. Here we abbreviate $\mathcal{D}^{\varepsilon}:=\mathcal{D}_{(u, A)}^{\varepsilon}$ for $\varepsilon \geq 0$.

Proof. We prove first that, for every vector field $v \in \operatorname{Vect}(\Sigma)$, there exists a constant $c=c\left(p, c_{0}, v\right)>0$ such that

$$
\begin{aligned}
\left\|\pi_{u} \tilde{\nabla}_{A, v} \xi-\tilde{\nabla}_{A, v} \pi_{u} \xi\right\|_{W^{k, p}, A} & \leq c\|\xi\|_{W^{k, p}, A}, \\
\left\|\pi_{u} \tilde{\nabla}_{A, v} \xi-\pi_{u} \tilde{\nabla}_{A, v} \pi_{u} \xi\right\|_{W^{k, p}, A} & \leq c\left\|\xi-\pi_{u} \xi\right\|_{W^{k, p}, A},
\end{aligned}
$$

for $(u, A) \in \widetilde{\mathcal{M}}_{B, \Sigma}^{0}\left(c_{0}\right), \xi \in \Omega^{0}\left(\Sigma, u^{*} T M / \mathrm{G}\right)$, and $k=0,1$. Here the $W^{1, p_{-}}$ norm labelled by $A$ is understood as the (gauge-invariant) $(1, p, \varepsilon)$-norm for $\varepsilon=1$. To prove equations (4.4), we choose local holomorphic coordinates $s+i t$ on $\Sigma$. Thus $\xi(s, t) \in T_{u(s, t)} M$, and $v_{s}, v_{t}, \nabla_{A, s} \xi$, and $\nabla_{A, t} \xi$ are as in Remark 4.1. Write

$$
\xi=\pi_{u} \xi+L_{u} \eta_{1}+J L_{u} \eta_{2},
$$

where $\eta_{i}(s, t) \in \mathfrak{g}$. Define $B_{s}(s, t): \mathfrak{g} \rightarrow T_{u(s, t)} M$ and $B_{t}(s, t): \mathfrak{g} \rightarrow T_{u(s, t)} M$ by

$$
B_{s} \eta:=\nabla_{v_{s}} X_{\eta}(u), \quad B_{t} \eta:=\nabla_{v_{t}} X_{\eta}(u)
$$


Then

$$
\nabla_{A, s} L_{u} \eta-L_{u} \nabla_{A, s} \eta=B_{s} \eta, \quad \nabla_{A, t} L_{u} \eta-L_{u} \nabla_{A, t} \eta=B_{t} \eta,
$$

and hence

$$
\nabla_{A, s} \pi_{u} \xi-\pi_{u} \nabla_{A, s} \xi=\pi_{u}\left(B_{s} \eta_{1}+J B_{s} \eta_{2}+\left(\nabla_{v_{s}} J+\partial_{s} J\right) L_{u} \eta_{2}\right) .
$$

Since $\eta_{1}=\left(L_{u}^{*} L_{u}\right)^{-1} L_{u}^{*}\left(\xi-\pi_{u} \xi\right)$ and $\eta_{2}=-\left(L_{u}^{*} L_{u}\right)^{-1} L_{u}^{*} J\left(\xi-\pi_{u} \xi\right)$, we have

$$
\left\|\nabla_{A, s} \pi_{u} \xi-\pi_{u} \nabla_{A, s} \xi\right\|_{L^{p}} \leq c\left\|\xi-\pi_{u} \xi\right\|_{L^{p}} .
$$

This proves equations (4.4) for the local vector field $\partial / \partial s$. For $\partial / \partial t$ the proof is analogous. Hence the result follows for any linear combination of these vector fields supported in the given coordinate chart, and hence for every vector field on $\Sigma$. For $\xi^{\prime} \in \Omega^{0,1}\left(\Sigma, u^{*} T M / \mathrm{G}\right)$ there are similar inequalities.

By equations (4.4), there exists a constant $c^{\prime}=c^{\prime}\left(p, c_{0}\right)>0$ such that

$$
\begin{aligned}
\left\|\pi_{u}\left(\tilde{\nabla}_{A} \xi\right)^{0,1}-\left(\tilde{\nabla}_{A, v} \pi_{u} \xi\right)^{0,1}\right\|_{W^{k, p}, A} & \leq c^{\prime}\|\xi\|_{W^{k, p}, A}, \\
\left\|\pi_{u}\left(\tilde{\nabla}_{A} \xi\right)^{0,1}-\pi_{u}\left(\tilde{\nabla}_{A, v} \pi_{u} \xi\right)^{0,1}\right\|_{W^{k, p}, A} & \leq c^{\prime}\left\|\xi-\pi_{u} \xi\right\|_{W^{k, p}, A},
\end{aligned}
$$

for $(u, A) \in \widetilde{\mathcal{M}}_{B, \Sigma}^{0}\left(c_{0}\right), \xi \in \Omega^{0}\left(\Sigma, u^{*} T M / \mathrm{G}\right)$, and $k=0$, 1 . Since

$$
D \xi:=D_{(u, A)} \xi=\left(\nabla_{A} \xi\right)^{0,1}-J\left(\nabla_{\xi} J\right) \partial_{J, A}(u),
$$

we have

$$
\begin{aligned}
\left\|\pi_{u} D \xi-D \pi_{u} \xi\right\|_{W^{k, p}, A} & \leq c^{\prime \prime}\|\xi\|_{W^{k, p}, A}, \\
\left\|\pi_{u} D\left(\xi-\pi_{u} \xi\right)\right\|_{W^{k, p}, A} & \leq c^{\prime \prime}\left\|\xi-\pi_{u} \xi\right\|_{W^{k, p}, A},
\end{aligned}
$$

for $(u, A) \in \widetilde{\mathcal{M}}_{B, \Sigma}^{0}\left(c_{0}\right), \xi \in \Omega^{0}\left(\Sigma, u^{*} T M / \mathrm{G}\right)$, and $k=0$, 1. Since

$$
\pi_{u} \mathcal{D}^{\varepsilon} \zeta=\pi_{u} D \xi, \quad \mathcal{D}^{0} \pi_{u} \zeta=\pi_{u} D \pi_{u} \xi, \quad \mathcal{D}^{\varepsilon} \pi_{u} \zeta=\left(D \pi_{u} \xi, 0,0\right),
$$

the required estimates for the operator $\mathcal{D}^{\varepsilon}$ follow from equation (4.5). The proof for the adjoint operator is analogous.

In the following, we use the notation

$$
\tilde{\nabla}_{A, v} \zeta:=\left(\tilde{\nabla}_{A, v} \xi, \nabla_{A, v} \alpha\right), \quad \tilde{\nabla}_{A, v} \zeta^{\prime}:=\left(\tilde{\nabla}_{A, v} \xi^{\prime}, \nabla_{A, v} \varphi^{\prime}, \nabla_{A, v} \psi^{\prime}\right)
$$

for $v \in \operatorname{Vect}(\Sigma), \zeta=(\xi, \alpha) \in \mathcal{X}_{u}$, and $\zeta^{\prime}=\left(\xi^{\prime}, \varphi^{\prime}, \psi^{\prime}\right) \in \mathcal{X}_{u}^{\prime}$, where $\tilde{\nabla}_{A}$ is the connection on $u^{*} T M / \mathrm{G}$ defined by equation (B.2) and $\nabla_{A, v} \alpha \in \Omega^{1}\left(\Sigma, \mathfrak{g}_{P}\right)$ is the covariant derivative induced by the connection $A$ and the Levi-Civita connection on $\Sigma$.

Lemma 4.6. For every $p \geq 2$, every $c_{0}>0$, and every equivariant vector field $v \in \operatorname{Vect}(\Sigma)$, there exists a constant $c>0$ such that

$$
\begin{aligned}
\left\|\mathcal{D}^{\varepsilon} \tilde{\nabla}_{A, v} \zeta-\tilde{\nabla}_{A, v} \mathcal{D}^{\varepsilon} \zeta\right\|_{k, p, \varepsilon} & \leq c \varepsilon^{-1}\|\zeta\|_{k+1, p, \varepsilon}, \\
\left\|\mathcal{D}^{\varepsilon *} \tilde{\nabla}_{A, v} \zeta^{\prime}-\tilde{\nabla}_{A, v} \mathcal{D}^{\varepsilon *} \zeta^{\prime}\right\|_{k, p, \varepsilon} & \leq c \varepsilon^{-1}\left\|\zeta^{\prime}\right\|_{k+1, p, \varepsilon},
\end{aligned}
$$

for all $(u, A) \in \widetilde{\mathcal{M}}_{B, \Sigma}^{0}\left(c_{0}\right), \zeta \in \mathcal{X}_{u}, \zeta^{\prime} \in \mathcal{X}_{u}, \varepsilon \in(0,1]$, and $k=0,1$. 
Proof. We compute in local coordinates. Let $\zeta^{\prime}=\left(\xi^{\prime}, \varphi^{\prime}, \psi^{\prime}\right):=\mathcal{D}^{\varepsilon} \zeta$. Then

$$
\xi^{\prime}=\tilde{\nabla}_{A, s} \xi+J \tilde{\nabla}_{A, t} \xi+\frac{1}{4} N\left(\xi, v_{s}-J v_{t}\right)+\frac{1}{2}\left(J \partial_{s} J-\partial_{t} J\right) \xi+L_{u} \varphi+J L_{u} \psi,
$$

$$
\begin{aligned}
& \varphi^{\prime}=\lambda^{-2}\left(\nabla_{A, s} \varphi+\nabla_{A, t} \psi\right)+\varepsilon^{-2} L_{u}^{*} \xi, \\
& \psi^{\prime}=\lambda^{-2}\left(\nabla_{A, s} \psi-\nabla_{A, t} \varphi\right)+\varepsilon^{-2} d \mu(u) \xi
\end{aligned}
$$

Here

$$
A=\Phi d s+\Psi d t, \quad \alpha=\varphi d s+\psi d t,
$$

and $v_{s}, v_{t}, \nabla_{A, s} \varphi, \tilde{\nabla}_{A, s} \xi, \nabla_{A, t} \varphi$, and $\tilde{\nabla}_{A, t} \xi$ are as in Remark 4.1. It suffices to prove the estimate for the local operators $\tilde{\nabla}_{A, s}$ and $\tilde{\nabla}_{A, t}$. Let $\zeta_{s}^{\prime}=\left(\xi_{s}^{\prime}, \varphi_{s}^{\prime}, \psi_{s}^{\prime}\right)$ be defined by equations (4.8) with $(\xi, \varphi, \psi)$ replaced by $\left(\tilde{\nabla}_{A, s} \xi, \nabla_{A, s} \varphi, \nabla_{A, s} \psi\right)$. Since $\tilde{\nabla}_{A} J=0$, we obtain

$$
\begin{aligned}
\tilde{\nabla}_{A, s} \xi^{\prime}-\xi_{s}^{\prime}= & J\left(\tilde{\nabla}_{A, s} \tilde{\nabla}_{A, t} \xi-\tilde{\nabla}_{A, t} \tilde{\nabla}_{A, s} \xi\right)+\nabla_{v_{s}} X_{\varphi}(u)+J \nabla_{v_{s}} X_{\psi}(u) \\
& +\frac{1}{4} \tilde{\nabla}_{A, s}\left(N\left(\xi, v_{s}-J v_{t}\right)\right)-\frac{1}{4} N\left(\tilde{\nabla}_{A, s} \xi, v_{s}-J v_{t}\right) \\
& +\frac{1}{2} \tilde{\nabla}_{A, s}\left(\left(J \partial_{s} J-\partial_{t} J\right) \xi\right)-\frac{1}{2}\left(J \partial_{s} J-\partial_{t} J\right) \tilde{\nabla}_{A, s} \xi \\
& -\frac{1}{2} J\left(\nabla_{v_{s}} J+\partial_{s} J\right)\left(L_{u} \varphi-J L_{u} \psi\right), \\
\nabla_{A, s} \varphi^{\prime}-\varphi_{s}^{\prime}= & \lambda^{-2}\left(\nabla_{A, s} \nabla_{A, t} \psi-\nabla_{A, t} \nabla_{A, s} \psi\right)+\left(\partial_{s} \lambda^{-2}\right)\left(\nabla_{A, s} \varphi+\nabla_{A, t} \psi\right) \\
& +\varepsilon^{-2} \rho\left(v_{s}, \xi\right)-\frac{1}{2} \varepsilon^{-2} d \mu(u)\left(\nabla_{v_{s}} J+\partial_{s} J\right) \xi \\
\nabla_{A, s} \psi^{\prime}-\psi_{s}^{\prime}= & -\lambda^{-2}\left(\nabla_{A, s} \nabla_{A, t} \varphi+\nabla_{A, t} \nabla_{A, s} \varphi\right)+\left(\partial_{s} \lambda^{-2}\right)\left(\nabla_{A, s} \psi-\nabla_{A, t} \varphi\right) \\
& -\varepsilon^{-2} \rho\left(v_{s}, J \xi\right)-\frac{1}{2} \varepsilon^{-2} L_{u}^{*}\left(\nabla_{v_{s}} J+\partial_{s} J\right) \xi
\end{aligned}
$$

Here we have used Lemma C.2. For the vector field $\partial / \partial s$, multiplied by any cutoff function, the estimates (4.6) and (4.7) follow from these three identities. The proof for $\partial / \partial t$ is similar, and so is the proof for the adjoint operator.

Lemma 4.7. Let $p \geq 2$ and $c_{0}>0$. Suppose that $\mathcal{D}^{0}:=\mathcal{D}_{(u, A)}^{0}$ is onto for every $(u, A) \in \widetilde{\mathcal{M}}_{B, \Sigma}^{0}\left(c_{0}\right)$. Then there exist positive constants $\varepsilon_{0}$ and $c$ such that the operator $\mathcal{D}^{\varepsilon}:=\mathcal{D}_{(u, A)}^{\varepsilon}$ is onto for every $(u, A) \in \widetilde{\mathcal{M}}_{B, \Sigma}^{0}\left(c_{0}\right)$ and every $\varepsilon \in\left(0, \varepsilon_{0}\right]$ and

$$
\begin{aligned}
\left\|\zeta^{\prime}\right\|_{k+1, p, \varepsilon} & \leq c\left(\varepsilon\left\|\mathcal{D}^{\varepsilon *} \zeta^{\prime}\right\|_{k, p, \varepsilon}+\left\|\pi_{u} \mathcal{D}^{\varepsilon *} \zeta^{\prime}\right\|_{k, p, \varepsilon}\right), \\
\left\|\zeta^{\prime}-\pi_{u} \zeta^{\prime}\right\|_{k+1, p, \varepsilon} & \leq c \varepsilon\left\|\mathcal{D}^{\varepsilon *} \zeta^{\prime}\right\|_{k, p, \varepsilon}
\end{aligned}
$$

for $\zeta^{\prime} \in \mathcal{X}_{u}^{\prime}$ and $k=0,1$. 
Proof. By elliptic regularity, there exists a constant $C_{0}>0$ such that

$$
\left\|\xi_{0}\right\|_{L^{p}} \leq C_{0}\left\|\mathcal{D}^{0^{*}} \xi_{0}\right\|_{L^{p}}
$$

for every $(u, A) \in \widetilde{\mathcal{M}}_{B, \Sigma}^{0}\left(c_{0}\right)$ and every $\xi_{0}^{\prime} \in \Omega^{0,1}\left(\Sigma, H_{u}\right)$. Hence

$$
\begin{aligned}
\left\|\pi_{u} \zeta^{\prime}\right\|_{L^{p}} & \leq C_{0}\left\|\mathcal{D}^{0^{*}} \pi_{u} \zeta^{\prime}\right\|_{L^{p}} \\
& \leq C_{0}\left\|\pi_{u} \mathcal{D}^{\varepsilon *} \zeta^{\prime}\right\|_{L^{p}}+C_{0}\left\|\pi_{u} \mathcal{D}^{\varepsilon *} \zeta^{\prime}-\mathcal{D}^{0^{*}} \pi_{u} \zeta^{\prime}\right\|_{L^{p}} \\
& \leq C_{0}\left\|\pi_{u} \mathcal{D}^{\varepsilon *} \zeta^{\prime}\right\|_{L^{p}}+C_{0} c_{1}\left\|\zeta^{\prime}-\pi_{u} \zeta^{\prime}\right\|_{0, p, \varepsilon} \\
& \leq C_{0}\left\|\pi_{u} \mathcal{D}^{\varepsilon *} \zeta^{\prime}\right\|_{L^{p}}+C_{0} c_{1} c_{2} \varepsilon\left(\left\|\mathcal{D}^{\varepsilon *} \zeta^{\prime}\right\|_{0, p, \varepsilon}+\left\|\pi_{u} \zeta^{\prime}\right\|_{L^{p}}\right) .
\end{aligned}
$$

Here $c_{1}$ is the constant of Lemma 4.5 and $c_{2}$ is the constant of Proposition 4.2. With $C_{0} c_{1} c_{2} \varepsilon \leq 1 / 2$, we obtain

$$
\left\|\pi_{u} \zeta^{\prime}\right\|_{L^{p}} \leq c_{3}\left(\varepsilon\left\|\mathcal{D}^{\varepsilon *} \zeta^{\prime}\right\|_{0, p, \varepsilon}+\left\|\pi_{u} \mathcal{D}^{\varepsilon *} \zeta^{\prime}\right\|_{L^{p}}\right),
$$

where $c_{3}:=2 C_{0} c_{1} c_{2}$. The inequality (4.9) for $k=0$ now follows from equation (4.12) and Proposition 4.2. To prove equation (4.10) for $k=0$ we use Proposition 4.2 and equation (4.12) again to obtain

$$
\begin{aligned}
\left\|\zeta^{\prime}-\pi_{u} \zeta^{\prime}\right\|_{1, p, \varepsilon} & \leq c_{2} \varepsilon\left(\left\|\mathcal{D}^{\varepsilon *} \zeta^{\prime}\right\|_{0, p, \varepsilon}+\left\|\pi_{u} \zeta^{\prime}\right\|_{L^{p}}\right) \\
& \leq c_{2} \varepsilon\left(1+c_{3} \varepsilon\right)\left\|\mathcal{D}^{\varepsilon *} \zeta^{\prime}\right\|_{0, p, \varepsilon}+c_{2} c_{3} \varepsilon\left\|\pi_{u} \mathcal{D}^{\varepsilon *} \zeta^{\prime}\right\|_{L^{p}} \\
& \leq c_{4} \varepsilon\left\|\mathcal{D}^{\varepsilon *} \zeta^{\prime}\right\|_{0, p, \varepsilon}
\end{aligned}
$$

where $c_{4}:=c_{2}\left(1+2 c_{3}\right)$.

Now let $v \in \operatorname{Vect}(\Sigma)$. By definition of the $(1, p, \varepsilon)$-norm and equations (4.4), there exists a constant $c_{5}=c_{5}\left(v, p, c_{0}\right)$ such that

$$
\left\|\tilde{\nabla}_{A, v} \zeta\right\|_{0, p, \varepsilon} \leq c_{5} \varepsilon^{-1}\|\zeta\|_{1, p, \varepsilon}, \quad\left\|\pi_{u} \tilde{\nabla}_{A, v} \zeta-\tilde{\nabla}_{A, v} \pi_{u} \zeta\right\|_{L^{p}} \leq c_{5}\|\zeta\|_{0, p, \varepsilon}
$$

for $(u, A) \in \widetilde{\mathcal{M}}_{B, \Sigma}^{0}\left(c_{0}\right), \zeta \in \mathcal{X}_{u}$, and $\varepsilon \in(0,1]$. Let $c_{6}=c_{6}\left(v, p, c_{0}\right)$ be the constant of Lemma 4.6. Then, by equation (4.9) with $k=0$ and Lemma 4.6, we have

$$
\begin{aligned}
\varepsilon\left\|\tilde{\nabla}_{A, v} \zeta^{\prime}\right\|_{1, p, \varepsilon} \leq & c \varepsilon\left(\varepsilon\left\|\mathcal{D}^{\varepsilon *} \tilde{\nabla}_{A, v} \zeta^{\prime}\right\|_{0, p, \varepsilon}+\left\|\pi_{u} \mathcal{D}^{\varepsilon *} \tilde{\nabla}_{A, v} \zeta^{\prime}\right\|_{L^{p}}\right) \\
\leq & c \varepsilon^{2}\left\|\mathcal{D}^{\varepsilon *} \tilde{\nabla}_{A, v} \zeta^{\prime}-\tilde{\nabla}_{A, v} \mathcal{D}^{\varepsilon *} \zeta^{\prime}\right\|_{0, p, \varepsilon}+c \varepsilon^{2}\left\|\tilde{\nabla}_{A, v} \mathcal{D}^{\varepsilon *} \zeta^{\prime}\right\|_{0, p, \varepsilon} \\
& +c \varepsilon\left\|\pi_{u}\left(\mathcal{D}^{\varepsilon *} \tilde{\nabla}_{A, v} \zeta^{\prime}-\tilde{\nabla}_{A, v} \mathcal{D}^{\varepsilon *} \zeta^{\prime}\right)\right\|_{L^{p}} \\
& +c \varepsilon\left\|\pi_{u} \tilde{\nabla}_{A, v} \mathcal{D}^{\varepsilon *} \zeta^{\prime}-\tilde{\nabla}_{A, v} \pi_{u} \mathcal{D}^{\varepsilon *} \zeta^{\prime}\right\|_{L^{p}}+c \varepsilon\left\|\tilde{\nabla}_{A, v} \pi_{u} \mathcal{D}^{\varepsilon *} \zeta^{\prime}\right\|_{L^{p}} \\
\leq & 2 c c_{5}\left(\varepsilon\left\|\mathcal{D}^{\varepsilon *} \zeta^{\prime}\right\|_{1, p, \varepsilon}+\left\|\pi_{u} \mathcal{D}^{\varepsilon *} \zeta^{\prime}\right\|_{1, p, \varepsilon}\right)+2 c c_{6}\left\|\zeta^{\prime}\right\|_{1, p, \varepsilon} \\
\leq & 2 c\left(c_{5}+c c_{6}\right)\left(\varepsilon\left\|\mathcal{D}^{\varepsilon *} \zeta^{\prime}\right\|_{1, p, \varepsilon}+\left\|\pi_{u} \mathcal{D}^{\varepsilon *} \zeta^{\prime}\right\|_{1, p, \varepsilon}\right)
\end{aligned}
$$

The last inequality follows again from equation (4.9) with $k=0$. The estimate (4.9) for $k=1$ now follows by taking the sum over finitely many suitably chosen vector fields $v$. 
To prove equation (4.11) for $k=1$ we observe that $\pi_{u} \mathcal{D}^{\varepsilon *} \pi_{u} \zeta^{\prime}=\mathcal{D}^{0^{*}} \pi_{u} \zeta^{\prime}$ and choose $c_{7}$ such that $\left\|\pi_{u} \zeta\right\|_{1, p, \varepsilon} \leq c_{7}\|\zeta\|_{1, p, \varepsilon}$ for every $\zeta \in \mathcal{X}_{u}$. Let $c_{8}$ be the constant of Lemma 4.5. Then, by equation (4.9) with $k=1$ and Lemma 4.5, we have

$$
\begin{aligned}
\left\|\zeta^{\prime}-\pi_{u} \zeta^{\prime}\right\|_{2, p, \varepsilon} \leq & c\left(\varepsilon\left\|\mathcal{D}^{\varepsilon *}\left(\zeta^{\prime}-\pi_{u} \zeta^{\prime}\right)\right\|_{1, p, \varepsilon}+\left\|\pi_{u} \mathcal{D}^{\varepsilon *}\left(\zeta^{\prime}-\pi_{u} \zeta^{\prime}\right)\right\|_{1, p, \varepsilon}\right) \\
\leq & c \varepsilon\left(\left\|\mathcal{D}^{\varepsilon *} \zeta^{\prime}\right\|_{1, p, \varepsilon}+\left\|\pi_{u} \mathcal{D}^{\varepsilon *} \zeta^{\prime}\right\|_{1, p, \varepsilon}\right)+c \varepsilon \| \mathcal{D}^{\varepsilon *} \pi_{u} \zeta^{\prime} \\
& -\pi_{u} \mathcal{D}^{\varepsilon *} \zeta^{\prime}\left\|_{1, p, \varepsilon}+c\right\| \pi_{u} \mathcal{D}^{\varepsilon *} \zeta^{\prime}-\mathcal{D}^{0^{*}} \pi_{u} \zeta^{\prime} \|_{1, p, \varepsilon} \\
\leq & c\left(1+c_{7}\right) \varepsilon\left\|\mathcal{D}^{\varepsilon *} \zeta^{\prime}\right\|_{1, p, \varepsilon}+c c_{8} \varepsilon \zeta^{\prime}\left\|_{1, p, \varepsilon}+c c_{8}\right\| \zeta^{\prime}-\pi_{u} \zeta^{\prime} \|_{1, p, \varepsilon} \\
\leq & c_{9} \varepsilon\left\|\mathcal{D}^{\varepsilon *} \zeta^{\prime}\right\|_{1, p, \varepsilon} .
\end{aligned}
$$

The last inequality follows from equations (4.9) and (4.10) with $k=0$.

Lemma 4.8. Let $p \geq 2$ and $c_{0}>0$. Suppose that $\mathcal{D}^{0}:=\mathcal{D}_{(u, A)}^{0}$ is onto for every $(u, A) \in \widetilde{\mathcal{M}}_{B, \Sigma}^{0}\left(c_{0}\right)$. Then there exist positive constants $c$ and $\varepsilon_{0}$ such that

$$
\left\|\mathcal{D}^{\varepsilon *} \zeta^{\prime}\right\|_{k+1, p, \varepsilon} \leq c\left(\varepsilon\left\|\mathcal{D}^{\varepsilon} \mathcal{D}^{\varepsilon *} \zeta^{\prime}\right\|_{k, p, \varepsilon}+\left\|\pi_{u} \mathcal{D}^{\varepsilon} \mathcal{D}^{\varepsilon *} \zeta^{\prime}\right\|_{k, p, \varepsilon}\right)
$$

$$
\left\|\mathcal{D}^{\varepsilon *} \zeta^{\prime}-\pi_{u} \mathcal{D}^{\varepsilon *} \zeta^{\prime}\right\|_{k+1, p, \varepsilon} \leq c \varepsilon\left\|\mathcal{D}^{\varepsilon} \mathcal{D}^{\varepsilon *} \zeta^{\prime}\right\|_{k, p, \varepsilon}
$$

for every $(u, A) \in \widetilde{\mathcal{M}}_{B, \Sigma}^{0}\left(c_{0}\right), \zeta^{\prime} \in \mathcal{X}_{u}^{\prime}, \varepsilon \in\left(0, \varepsilon_{0}\right]$, and $k=0,1$.

Proof. The proof has nine steps.

Step 1. Let $q>1$ such that $1 / p+1 / q=1$. Then there exists a constant $c_{0}>0$ such that

$$
\left\|\xi_{0}\right\|_{L^{p}}+\left\|\xi_{0}\right\|_{L^{q}} \leq c_{0}\left\|\xi_{0}\right\|_{L^{2}}, \quad\left\|\xi_{0}^{\prime}\right\|_{L^{q}} \leq c_{0}\left\|\mathcal{D}^{0^{*}} \xi_{0}^{\prime}\right\|_{L^{q}}
$$

for every $(u, A) \in \widetilde{\mathcal{M}}_{B, \Sigma}^{0}\left(c_{0}\right)$, every $\xi_{0} \in \operatorname{ker} \mathcal{D}^{0}$, and every $\xi_{0}^{\prime} \in \Omega^{0,1}\left(\Sigma, H_{u}\right)$.

These are standard estimates for elliptic PDEs. The first estimate uses $L^{2}$ regularity for the operator $\mathcal{D}^{0}$, the Sobolev embedding $W^{1,2} \hookrightarrow L^{p}$, and the Hölder inequality. The second estimate uses $L^{q}$ regularity for $\mathcal{D}^{0^{*}}$ and the fact that $\mathcal{D}^{0^{*}}$ is injective.

Step 2. There exists a constant $c_{1}>0$ such that

$$
\left\|\mathcal{D}^{0^{*}} \xi_{0}^{\prime}\right\|_{L^{p}} \leq c_{1} \sup _{\xi_{0}^{\prime \prime} \neq 0} \frac{\left\langle\mathcal{D}^{0^{*}} \xi_{0}^{\prime}, \mathcal{D}^{0^{*}} \xi_{0}^{\prime \prime}\right\rangle}{\left\|\mathcal{D}^{0^{*}} \xi_{0}^{\prime \prime}\right\|_{L^{q}}}
$$

for every $(u, A) \in \widetilde{\mathcal{M}}_{B, \Sigma}^{0}\left(c_{0}\right)$ and every $\xi_{0}^{\prime} \in \Omega^{0,1}\left(\Sigma, H_{u}\right)$. 
Let $\xi_{1}, \ldots, \xi_{m}$ be an $L^{2}$-orthonormal basis of ker $\mathcal{D}^{0}$. Given $\xi_{0}^{\prime}$ choose $\xi \in$ $L^{q}\left(\Sigma, H_{u}\right)$ such that

$$
\left\langle\xi, \mathcal{D}^{0^{*}} \xi_{0}^{\prime}\right\rangle=\left\|\mathcal{D}^{0^{*}} \xi_{0}^{\prime}\right\|_{L^{p}}, \quad\|\xi\|_{L^{q}}=1 .
$$

Let $\xi_{0}^{\prime \prime} \in W^{1, q}\left(\Sigma, \Lambda^{0,1} T^{*} \Sigma \otimes H_{u}\right)$ be the unique section such that

$$
\xi=\mathcal{D}^{0 *} \xi_{0}^{\prime \prime}+\sum_{j=1}^{m}\left\langle\xi, \xi_{j}\right\rangle \xi_{j} .
$$

Then

$$
\begin{aligned}
\left\|\mathcal{D}^{0^{*}} \xi_{0}^{\prime}\right\|_{L^{p}} & =\left\langle\mathcal{D}^{0^{*}} \xi_{0}^{\prime \prime}, \mathcal{D}^{0^{*}} \xi_{0}^{\prime}\right\rangle \\
& =\left\|\xi-\sum_{j=1}^{m}\left\langle\xi, \xi_{j}\right\rangle \xi_{j}\right\|_{L^{q}} \frac{\left\langle\mathcal{D}^{0^{*}} \xi_{0}^{\prime \prime}, \mathcal{D}^{0^{*}} \xi_{0}^{\prime}\right\rangle}{\left\|\mathcal{D}^{0^{*}} \xi_{0}^{\prime \prime}\right\|_{L^{q}}} \\
& \leq\left(1+\sum_{j=1}^{m}\left\|\xi_{j}\right\|_{L^{p}}\left\|\xi_{j}\right\|_{L^{q}}\right) \frac{\left\langle\mathcal{D}^{0^{*}} \xi_{0}^{\prime \prime}, \mathcal{D}^{0^{*}} \xi_{0}^{\prime}\right\rangle}{\left\|\mathcal{D}^{0^{*}} \xi_{0}^{\prime \prime}\right\|_{L^{q}}} \\
& \leq\left(1+m c_{0}{ }^{2}\right) \frac{\left\langle\mathcal{D}^{0^{*}} \xi_{0}^{\prime \prime}, \mathcal{D}^{0^{*}} \xi_{0}^{\prime}\right\rangle}{\left\|\mathcal{D}^{0^{*}} \xi_{0}^{\prime \prime}\right\|_{L^{q}}} .
\end{aligned}
$$

Step 3. There exists a constant $c_{2}>0$ such that

$$
\left\|\pi_{u} \mathcal{D}^{\varepsilon *} \zeta^{\prime}\right\|_{L^{p}} \leq c_{2}\left(\left\|\pi_{u} \mathcal{D}^{\varepsilon *} \zeta^{\prime}-\mathcal{D}^{0^{*}} \pi_{u} \zeta^{\prime}\right\|_{L^{p}}+\left\|\mathcal{D}^{0} \pi_{u} \mathcal{D}^{\varepsilon *} \zeta^{\prime}\right\|_{L^{p}}\right)
$$

for every $(u, A) \in \widetilde{\mathcal{M}}_{B, \Sigma}^{0}\left(c_{0}\right)$, every $\zeta^{\prime} \in \mathcal{X}_{u}^{\prime}$, and every $\varepsilon \in(0,1]$.

For every $\xi_{0}^{\prime} \in \Omega^{0,1}\left(\Sigma, H_{u}\right)$ we have

$$
\begin{aligned}
& \frac{\left\langle\mathcal{D}^{0^{*}} \xi_{0}^{\prime}, \mathcal{D}^{0^{*}} \pi_{u} \zeta^{\prime}\right\rangle}{\left\|\mathcal{D}^{0^{*}} \xi_{0}^{\prime}\right\|_{L^{q}}}=\frac{\left\langle\mathcal{D}^{0^{*}} \xi_{0}^{\prime}, \mathcal{D}^{0^{*}} \pi_{u} \zeta^{\prime}-\pi_{u} \mathcal{D}^{\varepsilon *} \zeta^{\prime}\right\rangle}{\left\|\mathcal{D}^{0^{*}} \xi_{0}^{\prime}\right\|_{L^{q}}}+\frac{\left\langle\xi_{0}^{\prime}, \mathcal{D}^{0} \pi_{u} \mathcal{D}^{\varepsilon *} \zeta^{\prime}\right\rangle}{\left\|\mathcal{D}^{0^{*}} \xi_{0}^{\prime}\right\|_{L^{q}}} \\
& \leq\left\|\mathcal{D}^{0^{*}} \pi_{u} \zeta^{\prime}-\pi_{u} \mathcal{D}^{\varepsilon *} \zeta^{\prime}\right\|_{L^{p}}+\left\|\mathcal{D}^{0} \pi_{u} \mathcal{D}^{\varepsilon *} \zeta^{\prime}\right\|_{L^{p}} \frac{\left\|\xi_{0}^{\prime}\right\|_{L^{q}}}{\left\|\mathcal{D}^{0^{*}} \xi_{0}^{\prime}\right\|_{L^{q}}} \\
& \leq\left\|\mathcal{D}^{0^{*}} \pi_{u} \zeta^{\prime}-\pi_{u} \mathcal{D}^{\varepsilon *} \zeta^{\prime}\right\|_{L^{p}}+c_{0}\left\|\mathcal{D}^{0} \pi_{u} \mathcal{D}^{\varepsilon *} \zeta^{\prime}\right\|_{L^{p}} .
\end{aligned}
$$

Here the last inequality follows from Step 1. Now, by Step 2,

$$
\begin{aligned}
\left\|\pi_{u} \mathcal{D}^{\varepsilon *} \zeta^{\prime}\right\|_{L^{p}} & \leq\left\|\mathcal{D}^{0^{*}} \pi_{u} \zeta^{\prime}-\pi_{u} \mathcal{D}^{\varepsilon *} \zeta^{\prime}\right\|_{L^{p}}+\left\|\mathcal{D}^{0^{*}} \pi_{u} \zeta^{\prime}\right\|_{L^{p}} \\
& \leq\left\|\mathcal{D}^{0^{*}} \pi_{u} \zeta^{\prime}-\pi_{u} \mathcal{D}^{\varepsilon *} \zeta^{\prime}\right\|_{L^{p}}+c_{1} \sup _{\xi_{0}^{\prime} \neq 0} \frac{\left\langle\mathcal{D}^{0^{*}} \pi_{u} \zeta^{\prime}, \mathcal{D}^{0^{*}} \xi_{0}^{\prime}\right\rangle}{\left\|\mathcal{D}^{0^{*}} \xi_{0}^{\prime}\right\|_{L^{q}}} \\
& \leq\left(1+c_{1}\right)\left\|\mathcal{D}^{0^{*}} \pi_{u} \zeta^{\prime}-\pi_{u} \mathcal{D}^{\varepsilon *} \zeta^{\prime}\right\|_{L^{p}}+c_{0} c_{1}\left\|\mathcal{D}^{0} \pi_{u} \mathcal{D}^{\varepsilon *} \zeta^{\prime}\right\|_{L^{p}}
\end{aligned}
$$


Step 4. There exist positive constants $\varepsilon_{0}$ and $c_{3}$ such that

$$
\left\|\pi_{u} \mathcal{D}^{\varepsilon *} \zeta^{\prime}\right\|_{L^{p}} \leq c_{3}\left(\varepsilon\left\|\mathcal{D}^{\varepsilon *} \zeta^{\prime}\right\|_{0, p, \varepsilon}+\varepsilon\left\|\mathcal{D}^{\varepsilon} \mathcal{D}^{\varepsilon *} \zeta^{\prime}\right\|_{0, p, \varepsilon}+\left\|\pi_{u} \mathcal{D}^{\varepsilon} \mathcal{D}^{\varepsilon *} \zeta^{\prime}\right\|_{L^{p}}\right)
$$

for every $(u, A) \in \widetilde{\mathcal{M}}_{B, \Sigma}^{0}\left(c_{0}\right)$, every $\zeta^{\prime} \in \mathcal{X}_{u}^{\prime}$, and every $\varepsilon \in\left(0, \varepsilon_{0}\right]$.

We apply Lemma 4.5 to both operators $\mathcal{D}^{\varepsilon}$ and $\mathcal{D}^{\varepsilon *}$. Then, by Step 3,

$$
\begin{aligned}
\left\|\pi_{u} \mathcal{D}^{\varepsilon *} \zeta^{\prime}\right\|_{L^{p}} \leq & c_{2}\left(\left\|\pi_{u} \mathcal{D}^{\varepsilon *} \zeta^{\prime}-\mathcal{D}^{0^{*}} \pi_{u} \zeta^{\prime}\right\|_{L^{p}}\right. \\
& \left.+\left\|\mathcal{D}^{0} \pi_{u} \mathcal{D}^{\varepsilon *} \zeta^{\prime}-\pi_{u} \mathcal{D}^{\varepsilon} \mathcal{D}^{\varepsilon *} \zeta^{\prime}\right\|_{0, p, \varepsilon}+\left\|\pi_{u} \mathcal{D}^{\varepsilon} \mathcal{D}^{\varepsilon *} \zeta^{\prime}\right\|_{L^{p}}\right) \\
\leq & c_{2}\left(c\left\|\zeta^{\prime}-\pi_{u} \zeta^{\prime}\right\|_{0, p, \varepsilon}+c\left\|\mathcal{D}^{\varepsilon *} \zeta^{\prime}-\pi_{u} \mathcal{D}^{\varepsilon *} \zeta^{\prime}\right\|_{0, p, \varepsilon}\right. \\
& \left.+\left\|\pi_{u} \mathcal{D}^{\varepsilon} \mathcal{D}^{\varepsilon *} \zeta^{\prime}\right\|_{L^{p}}\right) \\
\leq & c_{4}\left(\varepsilon\left\|\mathcal{D}^{\varepsilon *} \zeta^{\prime}\right\|_{0, p, \varepsilon}+\varepsilon\left\|\mathcal{D}^{\varepsilon} \mathcal{D}^{\varepsilon *} \zeta^{\prime}\right\|_{0, p, \varepsilon}+\left\|\pi_{u} \mathcal{D}^{\varepsilon} \mathcal{D}^{\varepsilon *} \zeta^{\prime}\right\|_{L^{p}}\right) .
\end{aligned}
$$

The last inequality follows from Lemma 4.7 and Proposition 4.2.

Step 5. We prove (4.13) for $k=0$.

By Proposition 4.2 and Step 4,

$$
\begin{aligned}
\left\|\mathcal{D}^{\varepsilon^{*}} \zeta^{\prime}\right\|_{1, p, \varepsilon} \leq & c\left(\varepsilon\left\|\mathcal{D}^{\varepsilon} \mathcal{D}^{\varepsilon *} \zeta^{\prime}\right\|_{1, p, \varepsilon}+\left\|\pi_{u} \mathcal{D}^{\varepsilon *} \zeta^{\prime}\right\|_{L^{p}}\right) \\
\leq & c\left(c_{3} \varepsilon\left\|\mathcal{D}^{\varepsilon *} \zeta^{\prime}\right\|_{0, p, \varepsilon}+\left(1+c_{3}\right) \varepsilon\left\|\mathcal{D}^{\varepsilon} \mathcal{D}^{\varepsilon *} \zeta^{\prime}\right\|_{0, p, \varepsilon}\right. \\
& \left.+c_{3}\left\|\pi_{u} \mathcal{D}^{\varepsilon} \mathcal{D}^{\varepsilon *} \zeta^{\prime}\right\|_{L^{p}}\right)
\end{aligned}
$$

for all $(u, A) \in \widetilde{\mathcal{M}}_{B, \Sigma}^{0}\left(c_{0}\right), \zeta^{\prime} \in \mathcal{X}_{u}^{\prime}$, and $\varepsilon \in\left(0, \varepsilon_{0}\right]$. With $c c_{3} \varepsilon \leq 1 / 2$ we obtain equation (4.13) for $k=0$.

Step 6. We prove (4.14) for $k=0$.

By Proposition 4.2 and Step 4,

$$
\begin{aligned}
\left\|\mathcal{D}^{\varepsilon *} \zeta^{\prime}-\pi_{u} \mathcal{D}^{\varepsilon *} \zeta^{\prime}\right\|_{1, p, \varepsilon} \leq & c \varepsilon\left(\left\|\mathcal{D}^{\varepsilon} \mathcal{D}^{\varepsilon *} \zeta^{\prime}\right\|_{1, p, \varepsilon}+\left\|\pi_{u} \mathcal{D}^{\varepsilon *} \zeta^{\prime}\right\|_{L^{p}}\right) \\
\leq & c \varepsilon\left(c_{3} \varepsilon\left\|\mathcal{D}^{\varepsilon *} \zeta^{\prime}\right\|_{0, p, \varepsilon}+\left(1+c_{3}\right) \varepsilon\left\|\mathcal{D}^{\varepsilon} \mathcal{D}^{\varepsilon *} \zeta^{\prime}\right\|_{0, p, \varepsilon}\right. \\
& \left.+c_{3}\left\|\pi_{u} \mathcal{D}^{\varepsilon} \mathcal{D}^{\varepsilon *} \zeta^{\prime}\right\|_{L^{p}}\right) \\
\leq & c_{5} \varepsilon\left\|\mathcal{D}^{\varepsilon} \mathcal{D}^{\varepsilon *} \zeta^{\prime}\right\|_{0, p, \varepsilon} .
\end{aligned}
$$

Here the last inequality follows from Step 5.

Step 7. There exist positive constants $\varepsilon_{0}$ and $c_{6}$ such that

$$
\begin{aligned}
\varepsilon^{2}\left\|\left[\mathcal{D}^{\varepsilon} \mathcal{D}^{\varepsilon *}, \tilde{\nabla}_{A, v}\right] \zeta^{\prime}\right\|_{L^{p}} & \leq c_{6}\left\|\zeta^{\prime}\right\|_{2, p, \varepsilon} \\
\varepsilon\left\|\left[\pi_{u} \mathcal{D}^{\varepsilon} \mathcal{D}^{\varepsilon *}, \tilde{\nabla}_{A, v}\right] \zeta^{\prime}\right\|_{L^{p}} & \leq c_{6}\left(\varepsilon\left\|\mathcal{D}^{\varepsilon} \mathcal{D}^{\varepsilon *} \zeta^{\prime}\right\|_{0, p, \varepsilon}+\left\|\pi_{u} \mathcal{D}^{\varepsilon} \mathcal{D}^{\varepsilon *} \zeta^{\prime}\right\|_{L^{p}}+\left\|\zeta^{\prime}\right\|_{1, p, \varepsilon}\right)
\end{aligned}
$$


for every $(u, A) \in \widetilde{\mathcal{M}}_{B, \Sigma}^{0}\left(c_{0}\right)$, every $\zeta^{\prime} \in \mathcal{X}_{u}^{\prime}$, and every $\varepsilon \in\left(0, \varepsilon_{0}\right]$.

The first estimate follows immediately from Lemma 4.6. To prove the second estimate, recall from Lemma 4.3 that

$$
\pi_{u} \mathcal{D}^{\varepsilon} \mathcal{D}^{\varepsilon *} \zeta^{\prime}=\pi_{u} 2 D D^{*} \xi^{\prime}+\pi_{u}(D J-J D) L_{u} \psi^{\prime},
$$

where $D: \Omega^{0}\left(\Sigma, u^{*} T M / \mathrm{G}\right) \rightarrow \Omega^{0,1}\left(\Sigma, u^{*} T M / \mathrm{G}\right)$ is the Cauchy-Riemann operator defined by equation (B.3) and $R:=(D J-J D) L_{u}$ is a zeroth-order operator (Lemma B.5). Hence

$$
2 D D^{*} \pi_{u} \xi^{\prime}=-2\left[\pi_{u}, D D^{*}\right] \xi^{\prime}+\pi_{u} \mathcal{D}^{\varepsilon} \mathcal{D}^{\varepsilon *} \zeta^{\prime}-\pi_{u} R \psi^{\prime} .
$$

By equations (4.5) in the proof of Lemma 4.5, the commutator $\left[\pi_{u}, D D^{*}\right]$ is a first-order operator in $\xi^{\prime}$. Hence there exists a constant $c_{7}=c_{7}\left(p, c_{0}\right)$ such that

$$
\left\|\tilde{\nabla}_{A}^{*} \tilde{\nabla}_{A} \pi_{u} \xi^{\prime}\right\|_{L^{p}} \leq c_{7}\left(\left\|\pi_{u} \mathcal{D}^{\varepsilon} \mathcal{D}^{\varepsilon *} \zeta^{\prime}\right\|_{L^{p}}+\varepsilon^{-1}\left\|\zeta^{\prime}\right\|_{1, p, \varepsilon}\right) .
$$

Moreover, by Lemma 4.7,

$$
\begin{aligned}
\left\|\tilde{\nabla}_{A}{ }^{*} \tilde{\nabla}_{A}\left(\xi^{\prime}-\pi_{u} \xi^{\prime}\right)\right\|_{L^{p}} & \leq \varepsilon^{-2}\left\|\xi^{\prime}-\pi_{u} \xi^{\prime}\right\|_{2, p, \varepsilon} \\
& \leq c_{8} \varepsilon^{-1}\left\|\mathcal{D}^{\varepsilon *} \zeta^{\prime}\right\|_{1, p, \varepsilon} \\
& \leq c_{9}\left(\left\|\mathcal{D}^{\varepsilon} \mathcal{D}^{\varepsilon *} \zeta^{\prime}\right\|_{0, p, \varepsilon}+\varepsilon^{-1}\left\|\pi_{u} \mathcal{D}^{\varepsilon} \mathcal{D}^{\varepsilon *} \zeta^{\prime}\right\|_{0, p, \varepsilon}\right) .
\end{aligned}
$$

The last inequality follows from Step 5 . Now the commutator

$$
\left[\tilde{\nabla}_{A, v}, \pi_{u} \mathcal{D}^{\varepsilon} \mathcal{D}^{\varepsilon *}\right] \zeta^{\prime}=2\left[\tilde{\nabla}_{A, v}, \pi_{u} D D^{*}\right] \xi^{\prime}+\left[\tilde{\nabla}_{A, v}, \pi_{u} R\right] \psi^{\prime}
$$

is a second-order operator in $\xi^{\prime}$ and a zeroth-order operator in $\psi^{\prime}$. Hence the assertion follows from the last two inequalities.

Step 8. We prove (4.13) for $k=1$.

Let $c_{10}$ be the constant in equation (4.13) for $k=0$ and $c_{11}$ be the constant of Lemma 4.6. Then, for every $v \in \operatorname{Vect}(\Sigma)$, we have

$$
\begin{aligned}
& \varepsilon\left\|\tilde{\nabla}_{A, v} \mathcal{D}^{\varepsilon *} \zeta^{\prime}\right\|_{1, p, \varepsilon} \\
& \leq \varepsilon\left\|\mathcal{D}^{\varepsilon *} \tilde{\nabla}_{A, v} \zeta^{\prime}\right\|_{1, p, \varepsilon}+\varepsilon\left\|\tilde{\nabla}_{A, v} \mathcal{D}^{\varepsilon *} \zeta^{\prime}-\mathcal{D}^{\varepsilon *} \tilde{\nabla}_{A, v} \zeta^{\prime}\right\|_{1, p, \varepsilon} \\
& \leq c_{10} \varepsilon^{2}\left\|\mathcal{D}^{\varepsilon} \mathcal{D}^{\varepsilon *} \tilde{\nabla}_{A, v} \zeta^{\prime}\right\|_{0, p, \varepsilon}+c_{10} \varepsilon\left\|\pi_{u} \mathcal{D}^{\varepsilon} \mathcal{D}^{\varepsilon *} \tilde{\nabla}_{A, v} \zeta^{\prime}\right\|_{L^{p}}+c_{11}\left\|\zeta^{\prime}\right\|_{2, p, \varepsilon} \\
& \leq c_{10} \varepsilon^{2}\left\|\tilde{\nabla}_{A, v} \mathcal{D}^{\varepsilon} \mathcal{D}^{\varepsilon *} \zeta^{\prime}\right\|_{0, p, \varepsilon}+c_{10} \varepsilon\left\|\tilde{\nabla}_{A, v} \pi_{u} \mathcal{D}^{\varepsilon} \mathcal{D}^{\varepsilon *} \zeta^{\prime}\right\|_{L^{p}} \\
&+c_{10} \varepsilon^{2}\left\|\left[\mathcal{D}^{\varepsilon} \mathcal{D}^{\varepsilon *}, \tilde{\nabla}_{A, v}\right] \zeta^{\prime}\right\|_{0, p, \varepsilon}+c_{10} \varepsilon\left\|\left[\pi_{u} \mathcal{D}^{\varepsilon} \mathcal{D}^{\varepsilon *}, \tilde{\nabla}_{A, v}\right] \zeta^{\prime}\right\|_{L^{p}}+c_{11}\left\|\zeta^{\prime}\right\|_{2, p, \varepsilon} \\
& \leq c_{10} \varepsilon^{2}\left\|\tilde{\nabla}_{A, v} \mathcal{D}^{\varepsilon} \mathcal{D}^{\varepsilon *} \zeta^{\prime}\right\|_{0, p, \varepsilon}+c_{10} \varepsilon\left\|\tilde{\nabla}_{A, v} \pi_{u} \mathcal{D}^{\varepsilon} \mathcal{D}^{\varepsilon *} \zeta^{\prime}\right\|_{L^{p}} \\
&+c_{6} c_{10}\left(\varepsilon\left\|\mathcal{D}^{\varepsilon} \mathcal{D}^{\varepsilon *} \zeta^{\prime}\right\|_{0, p, \varepsilon}+\left\|\pi_{u} \mathcal{D}^{\varepsilon} \mathcal{D}^{\varepsilon *} \zeta^{\prime}\right\|_{L^{p}}\right)+\left(2 c_{6} c_{10}+c_{11}\right)\left\|\zeta^{\prime}\right\|_{2, p, \varepsilon} \\
& \leq c_{12}\left(\varepsilon\left\|\mathcal{D}^{\varepsilon} \mathcal{D}^{\varepsilon *} \zeta^{\prime}\right\|_{1, p, \varepsilon}+\left\|\pi_{u} \mathcal{D}^{\varepsilon} \mathcal{D}^{\varepsilon *} \zeta^{\prime}\right\|_{1, p, \varepsilon}\right) .
\end{aligned}
$$


The penultimate inequality follows from Step 7, and the last step from equations (4.9) and (4.13) and the definition of the $(1, p, \varepsilon)$-norm. Now equation (4.13) for $k=1$ follows by taking the sum over finitely many vector fields $v \in \operatorname{Vect}(\Sigma)$.

Step 9. We prove (4.14) for $k=1$.

By Step 8, suppose that equation (4.13) holds with $k=1$ and $c=c_{13}$, choose $c_{14}$ such that $\left\|\pi_{u} \zeta\right\|_{1, p, \varepsilon} \leq c_{14}\|\zeta\|_{1, p, \varepsilon}$ for every $\zeta \in \mathcal{X}_{u}$, and let $c_{15}$ be the constant of Lemma 4.5. Then

$$
\begin{aligned}
\| \mathcal{D}^{\varepsilon *} & \zeta^{\prime}-\pi_{u} \mathcal{D}^{\varepsilon *} \zeta^{\prime} \|_{2, p, \varepsilon} \\
\leq & c_{13} \varepsilon\left\|\mathcal{D}^{\varepsilon}\left(\mathcal{D}^{\varepsilon *} \zeta^{\prime}-\pi_{u} \mathcal{D}^{\varepsilon *} \zeta^{\prime}\right)\right\|_{1, p, \varepsilon}+c_{13}\left\|\pi_{u} \mathcal{D}^{\varepsilon}\left(\mathcal{D}^{\varepsilon *} \zeta^{\prime}-\pi_{u} \mathcal{D}^{\varepsilon *} \zeta^{\prime}\right)\right\|_{1, p, \varepsilon} \\
\leq & c_{13} \varepsilon\left(\left\|\mathcal{D}^{\varepsilon} \mathcal{D}^{\varepsilon *} \zeta^{\prime}\right\|_{1, p, \varepsilon}+\left\|\pi_{u} \mathcal{D}^{\varepsilon} \mathcal{D}^{\varepsilon *} \zeta^{\prime}\right\|_{1, p, \varepsilon}\right) \\
& +c_{13} \varepsilon\left\|\mathcal{D}^{\varepsilon} \pi_{u} \mathcal{D}^{\varepsilon *} \zeta^{\prime}-\pi_{u} \mathcal{D}^{\varepsilon} \mathcal{D}^{\varepsilon *} \zeta^{\prime}\right\|_{1, p, \varepsilon}+c_{13}\left\|\left(\pi_{u} \mathcal{D}^{\varepsilon}-\mathcal{D}^{0} \pi_{u}\right) \mathcal{D}^{\varepsilon *} \zeta^{\prime}\right\|_{1, p, \varepsilon} \\
\leq & \left(1+c_{14}\right) c_{13} \varepsilon\left\|\mathcal{D}^{\varepsilon} \mathcal{D}^{\varepsilon *} \zeta^{\prime}\right\|_{1, p, \varepsilon} \\
& +c_{15} c_{13}\left(\varepsilon\left\|\mathcal{D}^{\varepsilon *} \zeta^{\prime}\right\|_{1, p, \varepsilon}+\left\|\mathcal{D}^{\varepsilon *} \zeta^{\prime}-\pi_{u} \mathcal{D}^{\varepsilon *} \zeta^{\prime}\right\|_{1, p, \varepsilon}\right) \\
\leq & c_{16} \varepsilon\left\|\mathcal{D}^{\varepsilon} \mathcal{D}^{\varepsilon *} \zeta^{\prime}\right\|_{1, p, \varepsilon} .
\end{aligned}
$$

The last inequality follows from Steps 5 and 6 .

\section{Quadratic estimates}

Fix $p>2, c_{0}>0$, and $(u, A) \in \widetilde{\mathcal{M}}_{B, \Sigma}^{0}\left(c_{0}\right)$, and consider the map

$$
\mathcal{F}^{\varepsilon}=\mathcal{F}_{(u, A)}^{\varepsilon}: \mathcal{X}_{u} \rightarrow \mathcal{X}_{u}^{\prime}
$$

given by

$$
\mathcal{F}^{\varepsilon}(\xi, \alpha)=\left(\begin{array}{c}
\rho(\xi)\left(\bar{\partial}_{J, A+\alpha}\left(\exp _{u}(\xi)\right)\right) \\
\varepsilon^{-2} L_{u}^{*} \xi-d_{A}^{*} \alpha \\
\varepsilon^{-2} \mu\left(\exp _{u}(\xi)\right)+* F_{A+\alpha}
\end{array}\right) .
$$

Here $\rho(\xi): T_{\exp _{u}(\xi)} M \rightarrow T_{u} M$ denotes parallel transport along the geodesic $r \mapsto \exp _{u}(r \xi)$ with respect to the Hermitian connection

$$
\tilde{\nabla}:=\nabla-\frac{1}{2} J \nabla J
$$

on $T M$. The differential of $\mathcal{F}^{\varepsilon}$ at zero is given by

$$
d \mathcal{F}^{\varepsilon}(0)=\mathcal{D}_{(u, A)}^{\varepsilon} .
$$

Let $\mathcal{F}_{i}^{\varepsilon}$ denote the $i$ th component of $\mathcal{F}^{\varepsilon}$. Since $\mathcal{F}_{2}^{\varepsilon}$ is a linear map, the following proposition only deals with the first and third components of $\mathcal{F}^{\varepsilon}$.

Proposition 5.1. For every $p>2$ and every $c_{0}>0$, there exists a constant $c>0$ such that the following holds for every $(u, A) \in \widetilde{\mathcal{M}}_{B, \Sigma}^{0}\left(c_{0}\right)$, any two pairs $\zeta=(\xi, \alpha), \hat{\zeta}=(\hat{\xi}, \hat{\alpha}) \in \mathcal{X}_{u}$, and every $\varepsilon \in(0,1]$. 
(i) If $\|\xi\|_{L^{\infty}}+\|\hat{\xi}\|_{L^{\infty}} \leq 1$, then

$$
\begin{aligned}
& \left\|\mathcal{F}_{1}^{\varepsilon}(\zeta+\hat{\zeta})-\mathcal{F}_{1}^{\varepsilon}(\zeta)-d \mathcal{F}_{1}^{\varepsilon}(\zeta) \hat{\zeta}\right\|_{L^{p}} \\
& \quad \leq c\|\hat{\xi}\|_{L^{\infty}}\left(\|\hat{\xi}\|_{L^{p}}+\left\|\tilde{\nabla}_{A} \hat{\xi}\right\|_{L^{p}}+\|\hat{\alpha}\|_{L^{p}}\right) \\
& \quad+c\|\hat{\xi}\|_{L^{\infty}}^{2}\left(\left\|\tilde{\nabla}_{A} \xi\right\|_{L^{p}}+\|\alpha\|_{L^{p}}+\left\|\tilde{\nabla}_{A} \hat{\xi}\right\|_{L^{p}}+\|\hat{\alpha}\|_{L^{p}}\right) .
\end{aligned}
$$

If, in addition, $\left\|\tilde{\nabla}_{A} \xi\right\|_{L^{p}}+\left\|\tilde{\nabla}_{A} \hat{\xi}\right\|_{L^{p}}+\|\alpha\|_{L^{p}}+\|\hat{\alpha}\|_{L^{p}} \leq \varepsilon^{2 / p-1}$, then

$$
\begin{aligned}
& \left\|\mathcal{F}_{1}^{\varepsilon}(\zeta+\hat{\zeta})-\mathcal{F}_{1}^{\varepsilon}(\zeta)-d \mathcal{F}_{1}^{\varepsilon}(\zeta) \hat{\zeta}\right\|_{L^{p}} \\
& \quad \leq c\|\hat{\xi}\|_{L^{\infty}}\left(\varepsilon^{-1}\|\hat{\xi}\|_{L^{p}}+\left\|\tilde{\nabla}{ }_{A} \hat{\xi}\right\|_{L^{p}}+\|\hat{\alpha}\|_{L^{p}}\right) .
\end{aligned}
$$

(ii) If $\|\xi\|_{L^{\infty}}+\|\hat{\xi}\|_{L^{\infty}} \leq 1$, then

$$
\begin{aligned}
& \left\|\mathcal{F}_{3}^{\varepsilon}(\zeta+\hat{\zeta})-\mathcal{F}_{3}^{\varepsilon}(\zeta)-d \mathcal{F}_{3}^{\varepsilon}(\zeta) \hat{\zeta}\right\|_{L^{p}} \\
& \quad \leq c\left(\|\hat{\alpha}\|_{L^{\infty}}\|\hat{\alpha}\|_{L^{p}}+\varepsilon^{-2}\|\hat{\xi}\|_{L^{\infty}}\|\hat{\xi}\|_{L^{p}}\right) .
\end{aligned}
$$

(iii) If $\|\xi\|_{L^{\infty}} \leq 1$, then

$$
\begin{aligned}
\left\|d \mathcal{F}_{1}^{\varepsilon}(\zeta) \hat{\zeta}-d \mathcal{F}_{1}^{\varepsilon}(0) \hat{\zeta}\right\|_{L^{p}} \leq & c\|\hat{\xi}\|_{L^{\infty}}\left(\|\xi\|_{L^{p}}+\left\|\tilde{\nabla}_{A} \xi\right\|_{L^{p}}+\|\alpha\|_{L^{p}}\right) \\
& +c\|\xi\|_{L^{\infty}}\left(\|\hat{\xi}\|_{L^{p}}+\left\|\tilde{\nabla}_{A} \hat{\xi}\right\|_{L^{p}}+\|\hat{\alpha}\|_{L^{p}}\right) .
\end{aligned}
$$

(iv) If $\|\xi\|_{L^{\infty}} \leq 1$, then

$$
\left\|d \mathcal{F}_{3}^{\varepsilon}(\zeta) \hat{\zeta}-d \mathcal{F}_{3}^{\varepsilon}(0) \hat{\zeta}\right\|_{L^{p}} \leq c\left(\varepsilon^{-2}\|\xi\|_{L^{p}}\|\hat{\xi}\|_{L^{\infty}}+\|\alpha\|_{L^{p}}\|\hat{\alpha}\|_{L^{\infty}}\right) .
$$

The estimates in Proposition 5.1 differ from the ones in $[6]$ in that the first derivatives of $\xi$ appear on the right-hand sides of the inequalities. This is because the nonlinearities in the Cauchy-Riemann equations appear in the first-order terms whereas the nonlinearities in the anti-self-duality equations only appear in the zeroth-order terms. In our equations, the nonlinear terms involving $\alpha$ are of zeroth-order. Hence no derivatives of $\alpha$ appear in the quadratic estimates. This is crucial for our adiabatic limit argument.

Proof of Proposition 5.1. In local holomorphic coordinates $s+i t$ on $\Sigma$, the map $\mathcal{F}^{\varepsilon}$ is given by

$$
\mathcal{F}^{\varepsilon}(\zeta)=\left(\begin{array}{c}
\rho(\xi)\left(\partial_{s} u_{\xi}+X_{\Phi+\varphi}\left(u_{\xi}\right)+J\left(\partial_{t} u_{\xi}+X_{\Psi+\psi}\left(u_{\xi}\right)\right)\right) \\
\varepsilon^{-2} L_{u}^{*} \xi+\lambda^{-2}\left(\nabla_{A, s} \varphi+\nabla_{A, t} \psi\right) \\
\varepsilon^{-2} \mu\left(u_{\xi}\right)+\lambda^{-2}\left(\partial_{s}(\Psi+\psi)-\partial_{t}(\Phi+\varphi)+[\Phi+\varphi, \Psi+\psi]\right)
\end{array}\right)
$$

where

Suppose that

$$
u_{\xi}:=\exp _{u}(\xi), \quad \zeta:=(\xi, \varphi, \psi), \quad \alpha:=\varphi d s+\psi d t .
$$

$$
\|\xi\|_{L^{\infty}} \leq 1
$$


The second derivatives of $\mathcal{F}_{1}^{\varepsilon}$ and $\mathcal{F}_{3}^{\varepsilon}$ satisfy the following pointwise estimates for suitable constants $c_{1}=c_{1}\left(u, A, v_{s}, v_{t}\right)$ and $c_{2}=c_{2}(u)$ (i.e., $c_{2}$ does not depend on the derivatives of $u$ ):

$$
\begin{aligned}
\left|d^{2} \mathcal{F}_{1}^{\varepsilon}(\zeta)\left(\zeta_{1}, \zeta_{2}\right)\right| \leq & c_{1}\left(\left(1+|\alpha|+\left|\tilde{\nabla}_{A} \xi\right|\right)\left|\xi_{1}\right|\left|\xi_{2}\right|\right. \\
& \left.+\left|\xi_{1}\right|\left(\left|\tilde{\nabla}_{A} \xi_{2}\right|+\left|\alpha_{2}\right|\right)+\left|\xi_{2}\right|\left(\left|\tilde{\nabla}_{A} \xi_{1}\right|+\left|\alpha_{1}\right|\right)\right), \\
\left|d^{2} \mathcal{F}_{3}^{\varepsilon}(\zeta)\left(\zeta_{1}, \zeta_{2}\right)\right| \leq & c_{2}\left(\left|\alpha_{1}\right|\left|\alpha_{2}\right|+\varepsilon^{-2}\left|\xi_{1}\right|\left|\xi_{2}\right|\right) .
\end{aligned}
$$

The estimate (5.3) is obvious and equation (5.2) follows from the fact that $\mathcal{F}_{1}^{\varepsilon}(\zeta)$ is linear and of zeroth-order in $\varphi$ and $\psi$ and that the first-order terms in $\xi$ are independent of $\varphi$ and $\psi$. Now consider the identities

$$
\begin{gathered}
\mathcal{F}^{\varepsilon}(\zeta+\hat{\zeta})-\mathcal{F}^{\varepsilon}(\zeta)-d \mathcal{F}^{\varepsilon}(\zeta) \hat{\zeta}=\int_{0}^{1}(1-r) d^{2} \mathcal{F}^{\varepsilon}(\zeta+r \hat{\zeta})(\hat{\zeta}, \hat{\zeta}) d r \\
d \mathcal{F}^{\varepsilon}(\zeta) \hat{\zeta}-d \mathcal{F}^{\varepsilon}(0) \hat{\zeta}=\int_{0}^{1} d^{2} \mathcal{F}^{\varepsilon}(r \zeta)(\zeta, \hat{\zeta}) d r
\end{gathered}
$$

To prove assertions (i) and (ii), replace $\left(\zeta, \zeta_{1}, \zeta_{2}\right)$ by $(\zeta+r \hat{\zeta}, \hat{\zeta}, \hat{\zeta})$ in equations (5.2) and (5.3), insert the resulting inequalities in equation (5.4), and integrate over $\Sigma$. Moreover, to derive the second assertion in (i) from the first we use the inequality $\varepsilon^{2 / p-1}\|\hat{\xi}\|_{L^{\infty}} \leq c\left(\varepsilon^{-1}\|\hat{\xi}\|_{L^{p}}+\left\|\tilde{\nabla} \tilde{\nabla}_{A} \hat{\xi}\right\|_{L^{p}}\right)$ of Lemma 4.4. To prove assertions (iii) and (iv), replace $\left(\zeta, \zeta_{1}, \zeta_{2}\right)$ by $(r \zeta, \zeta, \hat{\zeta})$ in equations (5.2) and (5.3), insert the resulting inequalities in equation (5.5), and integrate over $\Sigma$.

Proposition 5.2. For every $p>2$ and every $c_{0}>0$, there exists a constant $c>0$ such that the following holds for every $(u, A) \in \widetilde{\mathcal{M}}_{B, \Sigma}^{0}\left(c_{0}\right)$, any two pairs $\zeta=(\xi, \alpha), \hat{\zeta}=(\hat{\xi}, \hat{\alpha}) \in \mathcal{X}_{u}$, and every $\varepsilon \in(0,1]$.

(i) If $\|\xi\|_{L^{\infty}}+\|\hat{\xi}\|_{L^{\infty}} \leq 1$ and $\|\zeta\|_{1, p, \varepsilon}+\|\hat{\zeta}\|_{1, p, \varepsilon} \leq \varepsilon^{2 / p}$, then

$$
\left\|\mathcal{F}^{\varepsilon}(\zeta+\hat{\zeta})-\mathcal{F}^{\varepsilon}(\zeta)-d \mathcal{F}^{\varepsilon}(\zeta) \hat{\zeta}\right\|_{0, p, \varepsilon} \leq c \varepsilon^{-1-2 / p}\|\hat{\zeta}\|_{1, p, \varepsilon}^{2} .
$$

(ii) If $\|\xi\|_{L^{\infty}}+\|\hat{\xi}\|_{L^{\infty}} \leq 1$ and $\|\zeta\|_{2, p, \varepsilon}+\|\hat{\zeta}\|_{2, p, \varepsilon} \leq \varepsilon^{2 / p}$, then

$$
\left\|\mathcal{F}^{\varepsilon}(\zeta+\hat{\zeta})-\mathcal{F}^{\varepsilon}(\zeta)-d \mathcal{F}^{\varepsilon}(\zeta) \hat{\zeta}\right\|_{1, p, \varepsilon} \leq c \varepsilon^{-1-2 / p}\|\hat{\zeta}\|_{2, p, \varepsilon}^{2} .
$$

(iii) If $\|\xi\|_{L^{\infty}} \leq 1$, then

$$
\left\|d \mathcal{F}^{\varepsilon}(\zeta) \hat{\zeta}-d \mathcal{F}^{\varepsilon}(0) \hat{\zeta}\right\|_{0, p, \varepsilon} \leq c \varepsilon^{-1-2 / p}\|\zeta\|_{1, p, \varepsilon}\|\hat{\zeta}\|_{1, p, \varepsilon} .
$$

(iv) If $\|\xi\|_{L^{\infty}} \leq 1$ and $\|\zeta\|_{1, p, \varepsilon} \leq \varepsilon^{2 / p}$, then

$$
\left\|d \mathcal{F}^{\varepsilon}(\zeta) \hat{\zeta}-d \mathcal{F}^{\varepsilon}(0) \hat{\zeta}\right\|_{1, p, \varepsilon} \leq c \varepsilon^{-1-2 / p}\|\zeta\|_{2, p, \varepsilon}\|\hat{\zeta}\|_{2, p, \varepsilon} .
$$


Proof. Assertions (i) and (iii) follow immediately from Proposition 5.1. To prove (ii), we observe that in estimating the quadratic terms in $\partial \mathcal{F}_{1}^{\varepsilon}$ we encounter products of the following forms:

- $\partial^{2} \xi \cdot \hat{\xi} \cdot \hat{\xi}$ and $\partial \varphi \cdot \hat{\xi} \cdot \hat{\xi}$. Here the $L^{p}$-norms of $\partial^{2} \xi$ and $\partial \varphi$ can be estimated by $\varepsilon^{2 / p-2}$ and the $L^{\infty}$-norm of $\hat{\xi} \cdot \hat{\xi}$ by $\varepsilon^{-4 / p}\|\hat{\zeta}\|_{1, p, \varepsilon}^{2}$.

- $\xi \cdot \partial \hat{\xi} \cdot \partial \hat{\xi}, \xi \cdot \hat{\xi} \cdot \partial^{2} \hat{\xi}, \xi \cdot \partial \hat{\xi} \cdot \hat{\varphi}$, and $\xi \cdot \partial \hat{\varphi} \cdot \hat{\xi}$. The $L^{p}$-norms of these products can be estimated by $\varepsilon^{-2-2 / p}\|\hat{\zeta}\|_{1, p, \varepsilon}\|\hat{\zeta}\|_{2, p, \varepsilon}$.

- $\partial \xi \cdot \partial \hat{\xi} \cdot \hat{\xi}, \varphi \cdot \partial \hat{\xi} \cdot \hat{\xi}$, and $\partial \xi \cdot \hat{\varphi} \cdot \hat{\xi}$. In these cases the $L^{p}$-norm of $\partial \xi$ is bounded by $\varepsilon^{2 / p-1}$ and the $L^{\infty}$-norms of $\partial \hat{\xi} \cdot \hat{\xi}$ and $\hat{\varphi} \cdot \hat{\xi}$ are bounded by $\varepsilon^{-1-4 / p}\|\hat{\zeta}\|_{1, p, \varepsilon}\|\hat{\zeta}\|_{2, p, \varepsilon}$.

Similarly, in estimating the quadratic terms in $\partial \mathcal{F}_{3}^{\varepsilon}$ we encounter products of the following forms:

- $\varepsilon^{-2} \hat{\xi} \cdot \partial \hat{\xi}$ and $\partial \hat{\varphi} \cdot \hat{\psi}$. The $L^{p}$-norms of these products can be estimated by $\varepsilon^{-3-2 / p}\|\hat{\zeta}\|_{1, p, \varepsilon}^{2}$.

- $\varepsilon^{-2} \partial \xi \cdot \hat{\xi} \cdot \hat{\xi}$. Here the $L^{p}$-norm of $\partial \xi$ bounded by $\varepsilon^{2 / p-1}$ and the $L^{\infty}$-norm of $\varepsilon^{-2} \hat{\xi} \cdot \hat{\xi}$ is bounded by $\varepsilon^{-2-4 / p}\|\hat{\zeta}\|_{1, p, \varepsilon}^{2}$.

This proves (ii). The proof of (iv) is similar.

Assertions (i) and (iii) in Proposition 5.2 are weaker than Proposition 5.1; in the former, the first derivatives of $\alpha$ appear on the right-hand sides of the estimates. The full strength of Proposition 5.1 will be required in the proof of Theorem 6.2 .

\section{Proof of Theorem 3.1}

From now on, we assume (H1) and (H4). In this section, we establish the existence of a $\mathcal{G}(P)$-equivariant map $\widetilde{\mathcal{T}}^{\varepsilon}: \widetilde{\mathcal{M}}_{B, \Sigma}^{0}\left(c_{0}\right) \rightarrow \widetilde{\mathcal{M}}_{B, \Sigma}^{\varepsilon}$ that satisfies the requirements of Theorem 3.1.

Theorem 6.1. For every $c_{0}>0$ and every $p>2$, there exist positive constants $\varepsilon_{0}$ and $c$ such that for every $\varepsilon \in\left(0, \varepsilon_{0}\right]$ the following holds. For every $\left(u_{0}, A_{0}\right) \in \widetilde{\mathcal{M}}_{B, \Sigma}^{0}\left(c_{0}\right)$, there exists a unique pair $\zeta_{\varepsilon}=\left(\xi_{\varepsilon}, \alpha_{\varepsilon}\right) \in \mathcal{X}_{u_{0}}$ such that

$$
\begin{gathered}
\left(u_{\varepsilon}, A_{\varepsilon}\right):=\left(\exp _{u_{0}}\left(\xi_{\varepsilon}\right), A_{0}+\alpha_{\varepsilon}\right) \in \widetilde{\mathcal{M}}_{B, \Sigma}^{\varepsilon}, \\
-d_{A_{0}}^{*} \alpha_{\varepsilon}+\varepsilon^{-2} L_{u_{0}}^{*} \xi_{\varepsilon}=0, \quad \zeta_{\varepsilon} \in \operatorname{im}\left(\mathcal{D}_{(u, A)}^{\varepsilon}\right)^{*}, \\
\left\|\zeta_{\varepsilon}\right\|_{2, p, \varepsilon ;\left(u_{0}, A_{0}\right)} \leq c \varepsilon^{2} .
\end{gathered}
$$

The map $\left(u_{0}, A_{0}\right) \mapsto\left(u_{\varepsilon}, A_{\varepsilon}\right)$ is $\mathcal{G}(P)$-equivariant and will be denoted by $\widetilde{\mathcal{T}}^{\varepsilon}$.

The next theorem shows that uniqueness holds under a slightly weaker hypothesis, namely in a larger neighbourhood of $(u, A)$. 
Theorem 6.2. For every $c_{0}>0$ and every $p>2$, there exist positive constants $\delta$ and $\varepsilon_{0}$ such that for every $\varepsilon \in\left(0, \varepsilon_{0}\right]$ the following holds. Suppose that $\left(u_{0}, A_{0}\right) \in \widetilde{\mathcal{M}}_{B, \Sigma}^{0}\left(c_{0}\right)$ and $(\xi, \alpha) \in \mathcal{X}_{u_{0}}$ satisfy equations (6.1) and (6.2), and

$$
\|\xi\|_{1, p, \varepsilon}+\varepsilon\|\alpha\|_{L^{p}}+\varepsilon^{2 / p}\|(\xi, \alpha)\|_{\infty, \varepsilon} \leq \delta \varepsilon^{2 / p+1 / 2} .
$$

Then $\left(\exp _{u_{0}}(\xi), A_{0}+\alpha\right)=\widetilde{\mathcal{T}}^{\varepsilon}(u, A)$.

Corollary 6.3. For every $c_{0}>0$ and every $p>2$, there exist positive constants $\delta$ and $\varepsilon_{0}$ such that for every $\varepsilon \in\left(0, \varepsilon_{0}\right]$ the following holds. Suppose that $\left(u_{0}, A_{0}\right) \in \widetilde{\mathcal{M}}_{B, \Sigma}^{0}\left(c_{0}\right)$ and $\zeta=(\xi, \alpha) \in \mathcal{X}_{u_{0}}$ satisfy equations (6.1) and $(6.2)$, and

$$
\|\zeta\|_{1, p, \varepsilon ;\left(u_{0}, A_{0}\right)} \leq \delta \varepsilon^{2 / p+1 / 2} .
$$

Then $\left(\exp _{u_{0}}(\xi), A_{0}+\alpha\right)=\widetilde{\mathcal{T}}^{\varepsilon}(u, A)$.

Proof. Theorem 6.2 and Lemma 4.4.

Proof of Theorem 6.1. The proof is similar to that of Theorem 5.1 in [6]. However, in the present case the nonlinearities (in the quadratic estimates) appear in the highest-order terms, and we establish estimates for the $(2, p, \varepsilon)$ norms and not just the $(1, p, \varepsilon)$-norms (as in $[6]$ ). We assume throughout that the exponential map at each point in $\mu^{-1}(0)$ is defined in a ball of radius 1 .

Abbreviate $\mathcal{D}^{\varepsilon}:=\mathcal{D}_{\left(u_{0}, A_{0}\right)}^{\varepsilon}$ and let $\mathcal{F}^{\varepsilon}: \mathcal{X}_{u_{0}} \rightarrow \mathcal{X}_{u_{0}}^{\prime}$ be defined by equation (5.1). Then

$$
d \mathcal{F}^{\varepsilon}(0)=\mathcal{D}^{\varepsilon}, \quad \mathcal{F}^{\varepsilon}(0)=\left(0,0, * F_{A_{0}}\right) .
$$

Hence, by Lemma 2.2, there exists a constant $C_{0}>0$, depending only on $c_{0}$ and $p$, such that

$$
\left\|\mathcal{F}^{\varepsilon}(0)\right\|_{1, p, \varepsilon}=\varepsilon\left\|F_{A_{0}}\right\|_{L^{p}}+\varepsilon^{2}\left\|d_{A_{0}} * F_{A_{0}}\right\|_{L^{p}} \leq C_{0} \varepsilon .
$$

We use Newton iteration to obtain a zero of $\mathcal{F}^{\varepsilon}$, and hence a solution of equation (1.7). Let $\zeta_{\nu}=\left(\xi_{\nu}, \alpha_{\nu}\right) \in \mathcal{X}_{u_{0}}$ be the sequence defined recursively by $\zeta_{0}:=0$ and

$$
\zeta_{\nu+1}:=\zeta_{\nu}+\hat{\zeta}_{\nu}, \quad \hat{\zeta}_{\nu} \in \operatorname{im} \mathcal{D}^{\varepsilon *}, \quad \mathcal{D}^{\varepsilon} \hat{\zeta}_{\nu}=-\mathcal{F}^{\varepsilon}\left(\zeta_{\nu}\right) .
$$

We prove by induction over $\nu$ that there exist positive constants $\varepsilon_{0}, c_{1}, C$, depending only on $c_{0}$ and $p$, such that

$$
\begin{aligned}
\left\|\hat{\zeta}_{\nu}\right\|_{2, p, \varepsilon} & \leq c_{1}\left\|\mathcal{F}^{\varepsilon}\left(\zeta_{\nu}\right)\right\|_{1, p, \varepsilon} \\
\left\|\hat{\zeta}_{\nu}\right\|_{2, p, \varepsilon} & \leq 2^{-\nu} C_{0} c_{1} \varepsilon^{2} \\
\left\|\mathcal{F}^{\varepsilon}\left(\zeta_{\nu+1}\right)\right\|_{1, p, \varepsilon} & \leq C \varepsilon^{1-2 / p}\left\|\hat{\zeta}_{\nu}\right\|_{2, p, \varepsilon}
\end{aligned}
$$


for $\varepsilon \in\left(0, \varepsilon_{0}\right]$ and $\nu \geq 0$. The constants are chosen such that the linear estimates of Lemma 4.8 hold for $0<\varepsilon \leq \varepsilon_{0}$ with $c=c_{1} \geq 1$, the quadratic estimates of Proposition 5.2 hold for $0<\varepsilon \leq 1$ with $c=c_{2} \geq 1$, the $L^{\infty}$ estimates of Lemma 4.4 hold for $0<\varepsilon \leq 1$ with $c=c_{\infty} \geq 1$, and

$$
C=3 C_{0} c_{1} c_{2}, \quad C c_{1} \varepsilon_{0}^{1-2 / p} \leq 1 / 2, \quad 3 C_{0} c_{1} c_{\infty} \varepsilon_{0}^{2-2 / p} \leq 1 .
$$

For $\nu=0$, the estimates (6.7) and (6.8) follow from Lemma 4.8. Namely, by equation (4.13) with $k=1$, we have

$$
\left\|\hat{\zeta}_{0}\right\|_{2, p, \varepsilon} \leq c_{1} \varepsilon\left\|\mathcal{F}^{\varepsilon}(0)\right\|_{1, p, \varepsilon} \leq C_{0} c_{1} \varepsilon^{2} .
$$

The estimate (6.9) for $\nu=0$ follows from the identity $d \mathcal{F}^{\varepsilon}(0) \hat{\zeta}_{0}=-\mathcal{F}^{\varepsilon}(0)$ and assertion (ii) of Proposition 5.2. Namely, since $C_{0} c_{1} c_{\infty} \varepsilon^{2-2 / p} \leq 1$, we have

$$
\left\|\hat{\zeta}_{0}\right\|_{2, p, \varepsilon} \leq C_{0} c_{1} \varepsilon^{2} \leq \varepsilon^{2 / p}, \quad\left\|\hat{\zeta}_{0}\right\|_{\infty, \varepsilon} \leq C_{0} c_{1} c_{\infty} \varepsilon^{2-2 / p} \leq 1 .
$$

Hence the hypotheses of Proposition 5.2 (ii) are satisfied with $\zeta=0$ and $\hat{\zeta}=\hat{\zeta}_{0}$, and hence

$$
\begin{aligned}
\left\|\mathcal{F}^{\varepsilon}\left(\zeta_{1}\right)\right\|_{1, p, \varepsilon} & =\left\|\mathcal{F}^{\varepsilon}\left(\hat{\zeta}_{0}\right)-\mathcal{F}^{\varepsilon}(0)-d \mathcal{F}^{\varepsilon}(0) \hat{\zeta}_{0}\right\|_{1, p, \varepsilon} \\
& \leq c_{2} \varepsilon^{-1-2 / p}\left\|\hat{\zeta}_{0}\right\|_{2, p, \varepsilon}^{2} \\
& \leq C_{0} c_{1} c_{2} \varepsilon^{1-2 / p}\left\|\hat{\zeta}_{0}\right\|_{2, p, \varepsilon} .
\end{aligned}
$$

Since $C \geq C_{0} c_{1} c_{2}$, this proves equation (6.9) for $\nu=0$. Now assume that the sequences $\zeta_{0}, \ldots, \zeta_{\nu}$ and $\hat{\zeta}_{0}, \ldots, \hat{\zeta}_{\nu-1}$ have been constructed up to some integer $\nu \geq 1$ and that the estimates (6.7) to (6.9) have been established for all integers up to $\nu-1$. Then, by equation (6.8),

$$
\left\|\zeta_{\nu}\right\|_{2, p, \varepsilon} \leq \sum_{j=0}^{\nu-1}\left\|\zeta_{j+1}-\zeta_{j}\right\|_{2, p, \varepsilon}=\sum_{j=0}^{\nu-1}\left\|\hat{\zeta}_{j}\right\|_{2, p, \varepsilon} \leq 2 C_{0} c_{1} \varepsilon^{2}
$$

and hence

$$
\left\|\zeta_{\nu}\right\|_{\infty, \varepsilon} \leq 2 C_{0} c_{1} c_{\infty} \varepsilon^{2-2 / p} \leq 1
$$

This shows that $\xi_{\nu}(p)$ lies in the domain of the exponential map at $u_{0}(p)$ for every $p \in P$ and so $\zeta_{\nu}$ lies in the domain of $\mathcal{F}^{\varepsilon}$. Let $\hat{\zeta}_{\nu}$ and $\zeta_{\nu+1}$ be defined by equations (6.6). Then, by Lemma $4.8, \hat{\zeta}_{\nu}$ satisfies the estimate (6.7). To prove equation (6.8) we observe that, by the induction hypothesis,

$$
\begin{aligned}
\left\|\hat{\zeta}_{\nu}\right\|_{2, p, \varepsilon} & \leq c_{1}\left\|\mathcal{F}^{\varepsilon}\left(\zeta_{\nu}\right)\right\|_{1, p, \varepsilon} \\
& \leq C c_{1} \varepsilon^{1-2 / p}\left\|\hat{\zeta}_{\nu-1}\right\|_{2, p, \varepsilon} \\
& \leq 2^{-1}\left\|\hat{\zeta}_{\nu-1}\right\|_{2, p, \varepsilon} \\
& \leq 2^{-\nu} C_{0} c_{1} \varepsilon^{2}
\end{aligned}
$$


To prove equation (6.9) we observe that, by equation (6.10),

$$
\begin{aligned}
& \left\|\zeta_{\nu}\right\|_{2, p, \varepsilon}+\left\|\hat{\zeta}_{\nu}\right\|_{2, p, \varepsilon} \leq 3 C_{0} c_{1} \varepsilon^{2} \leq \varepsilon^{2 / p}, \\
& \left\|\zeta_{\nu}\right\|_{\infty, \varepsilon}+\left\|\hat{\zeta}_{\nu}\right\|_{\infty, \varepsilon} \leq 3 C_{0} c_{1} c_{\infty} \varepsilon^{2-2 / p} \leq 1 .
\end{aligned}
$$

Thus the hypotheses of Proposition 5.2 (ii) and (iv) are satisfied with $\zeta=\zeta_{\nu}$ and $\hat{\zeta}=\hat{\zeta}_{\nu}$. Hence

$$
\begin{aligned}
\left\|\mathcal{F}^{\varepsilon}\left(\zeta_{\nu+1}\right)\right\|_{1, p, \varepsilon} \leq & \left\|\mathcal{F}^{\varepsilon}\left(\zeta_{\nu}+\hat{\zeta}_{\nu}\right)-\mathcal{F}^{\varepsilon}\left(\zeta_{\nu}\right)-d \mathcal{F}^{\varepsilon}\left(\zeta_{\nu}\right) \hat{\zeta}_{\nu}\right\|_{1, p, \varepsilon} \\
& +\left\|d \mathcal{F}^{\varepsilon}\left(\zeta_{\nu}\right) \hat{\zeta}_{\nu}-d \mathcal{F}^{\varepsilon}(0) \hat{\zeta}_{\nu}\right\|_{1, p, \varepsilon} \\
\leq & c_{2} \varepsilon^{-1-2 / p}\left(\left\|\hat{\zeta}_{\nu}\right\|_{2, p, \varepsilon}+\left\|\zeta_{\nu}\right\|_{2, p, \varepsilon}\right)\left\|\hat{\zeta}_{\nu}\right\|_{2, p, \varepsilon} \\
\leq & 3 C_{0} c_{1} c_{2} \varepsilon^{1-2 / p}\left\|\hat{\zeta}_{\nu}\right\|_{2, p, \varepsilon} \\
= & C \varepsilon^{1-2 / p}\left\|\hat{\zeta}_{\nu}\right\|_{2, p, \varepsilon} .
\end{aligned}
$$

This completes the induction.

By equation (6.8), the sequence $\zeta_{\nu}$ is Cauchy in the $(2, p, \varepsilon)$-norm, and hence in the $W^{2, p}$-norm because $\varepsilon$ is fixed. Moreover, by examining the second component of $\mathcal{F}^{\varepsilon}$ we find that $\zeta_{\nu}$ satisfies equations (6.2) for every $\nu$ and hence so does its limit

$$
\zeta_{\varepsilon}:=\left(\xi_{\varepsilon}, \alpha_{\varepsilon}\right):=\lim _{\nu \rightarrow \infty}\left(\xi_{\nu}, \alpha_{\nu}\right) .
$$

By equation (6.10), this limit also satisfies equation (6.3) with $c:=2 C_{0} c_{1}$. Moreover, by equations (6.8) and (6.9), the sequence $\mathcal{F}^{\varepsilon}\left(\zeta_{\nu}\right)$ converges to zero in the $(1, p, \varepsilon)$-norm and hence $\mathcal{F}^{\varepsilon}\left(\zeta_{\varepsilon}\right)=0$. Hence $\zeta_{\varepsilon}$ satisfies equation (6.1) and it follows from elliptic regularity that $\zeta_{\varepsilon}$ is smooth. This proves existence.

We prove uniqueness. Suppose $\zeta=(\xi, \alpha) \in \mathcal{X}_{u_{0}}$ satisfies equations (6.1) and (6.2), and $\|\zeta\|_{1, p, \varepsilon} \leq c \varepsilon^{2}$. Then, by Lemma 4.8 and Proposition 5.2 (i) and (iii),

$$
\begin{aligned}
\left\|\zeta-\zeta_{\varepsilon}\right\|_{1, p, \varepsilon} \leq & c_{1}\left\|\mathcal{D}^{\varepsilon}\left(\zeta-\zeta_{\varepsilon}\right)\right\|_{0, p, \varepsilon} \\
\leq & c_{1}\left\|\mathcal{F}^{\varepsilon}(\zeta)-\mathcal{F}^{\varepsilon}\left(\zeta_{\varepsilon}\right)-d \mathcal{F}^{\varepsilon}\left(\zeta_{\varepsilon}\right)\left(\zeta-\zeta_{\varepsilon}\right)\right\|_{0, p, \varepsilon} \\
& +c_{1}\left\|d \mathcal{F}^{\varepsilon}(0)\left(\zeta-\zeta_{\varepsilon}\right)-d \mathcal{F}^{\varepsilon}\left(\zeta_{\varepsilon}\right)\left(\zeta-\zeta_{\varepsilon}\right)\right\|_{0, p, \varepsilon} \\
\leq & c_{1} c_{2} \varepsilon^{-1-2 / p}\left(\left\|\zeta-\zeta_{\varepsilon}\right\|_{1, p, \varepsilon}+\left\|\zeta_{\varepsilon}\right\|_{1, p, \varepsilon}\right)\left\|\zeta-\zeta_{\varepsilon}\right\|_{1, p, \varepsilon} \\
\leq & 3 c c_{1} c_{2} \varepsilon^{1-2 / p}\left\|\zeta-\zeta_{\varepsilon}\right\|_{1, p, \varepsilon} .
\end{aligned}
$$

If $3 c c_{1} c_{2} \varepsilon^{1-2 / p}<1$ then $\zeta=\zeta_{\varepsilon}$. This proves uniqueness.

Since the conditions (6.1), (6.2), and (6.3) are gauge-invariant, it follows that the map $(u, A) \mapsto\left(u_{\varepsilon}, A_{\varepsilon}\right)$ is $\mathcal{G}(P)$-equivariant.

Proof of Theorem 6.2. In this proof we drop the subscript 0. Fix two pairs $(u, A) \in \widetilde{\mathcal{M}}_{B, \Sigma}^{0}\left(c_{0}\right)$ and $\zeta=(\xi, \alpha) \in \mathcal{X}_{u}$ that satisfy equations 
(6.2) and (6.4), and

$$
\left(\exp _{u}(\xi), A+\alpha\right) \in \widetilde{\mathcal{M}}_{B, \Sigma}^{\varepsilon}
$$

We prove that $\zeta$ satisfies equation (6.3), provided that $\delta$ and $\varepsilon$ are sufficiently small. By ellipticity of the operator $\mathcal{D}^{0}:=\mathcal{D}_{(u, A)}^{0}$, there exists a constant $c_{1}=c_{1}\left(p, c_{0}\right)>0$ such that

$$
\left\|\tilde{\nabla}_{A} \pi_{u} \xi\right\|_{L^{p}} \leq c_{1}\left(\left\|\mathcal{D}^{0} \pi_{u} \xi\right\|_{L^{p}}+\left\|\pi_{u} \xi\right\|_{L^{p}}\right)
$$

Now let $c_{2}=c_{2}\left(p, c_{0}\right)$ be the constant of Lemma 4.5 and $c_{3}=c_{3}\left(p, c_{0}\right)$ be the constant of Proposition 4.2. Then

$$
\begin{aligned}
\left\|\tilde{\nabla}_{A} \xi\right\|_{L^{p}} \leq & \varepsilon^{-1}\left\|\xi-\pi_{u} \xi\right\|_{1, p, \varepsilon}+\left\|\tilde{\nabla}_{A} \pi_{u} \xi\right\|_{L^{p}} \\
\leq & \varepsilon^{-1}\left\|\xi-\pi_{u} \xi\right\|_{1, p, \varepsilon}+c_{1}\left(\left\|\mathcal{D}^{0} \pi_{u} \xi\right\|_{L^{p}}+\left\|\pi_{u} \xi\right\|_{L^{p}}\right) \\
\leq & \varepsilon^{-1}\left\|\xi-\pi_{u} \xi\right\|_{1, p, \varepsilon} \\
& +c_{1}\left(\left\|\left(\mathcal{D}^{0} \pi_{u}-\pi_{u} \mathcal{D}^{\varepsilon}\right) \xi\right\|_{L^{p}}+\left\|\pi_{u} \mathcal{D}^{\varepsilon} \zeta\right\|_{L^{p}}+\left\|\pi_{u} \xi\right\|_{L^{p}}\right) \\
\leq & \left(\varepsilon^{-1}+c_{1} c_{2}\right)\left\|\xi-\pi_{u} \xi\right\|_{1, p, \varepsilon}+c_{1}\left(\left\|\pi_{u} \mathcal{D}^{\varepsilon} \zeta\right\|_{L^{p}}+\left\|\pi_{u} \xi\right\|_{L^{p}}\right) \\
\leq & c_{4}\left(\left\|\mathcal{D}^{\varepsilon} \zeta\right\|_{0, p, \varepsilon}+\|\xi\|_{L^{p}}\right),
\end{aligned}
$$

where $c_{4}:=c_{3}\left(1+c_{1} c_{2}\right)+c_{1}$. Hence

$$
\begin{aligned}
\left\|\tilde{\nabla}_{A} \xi\right\|_{L^{p}}+\|\alpha\|_{L^{p}} & \leq c_{4}\left\|\mathcal{D}^{\varepsilon} \zeta\right\|_{0, p, \varepsilon}+c_{4}\|\xi\|_{L^{p}}+\varepsilon^{-1}\left\|\zeta-\pi_{u} \zeta\right\|_{1, p, \varepsilon} \\
& \leq\left(c_{4}+c_{5}\right)\left\|\mathcal{D}^{\varepsilon} \zeta\right\|_{0, p, \varepsilon}+c_{4}\|\xi\|_{L^{p}}
\end{aligned}
$$

where $c_{5}=c_{5}\left(p, c_{0}\right)$ is the constant of Lemma 4.8. Since

$$
\mathcal{F}^{\varepsilon}(\zeta)=0, \quad \mathcal{F}^{\varepsilon}(0)=\left(0,0, * F_{A}\right), \quad \mathcal{D}^{\varepsilon}=d \mathcal{F}^{\varepsilon}(0)
$$

we obtain

$$
\begin{aligned}
\left\|\tilde{\nabla}_{A} \xi\right\|_{L^{p}}+\|\alpha\|_{L^{p}} \leq & c_{6}\left\|\mathcal{F}^{\varepsilon}(\zeta)-\mathcal{F}^{\varepsilon}(0)-d \mathcal{F}^{\varepsilon}(0) \zeta\right\|_{0, p, \varepsilon} \\
& +c_{6} \varepsilon\left\|F_{A}\right\|_{L^{p}}+c_{4}\|\xi\|_{L^{p}}
\end{aligned}
$$

where $c_{6}:=c_{4}+c_{5}$. Now we use the refined quadratic estimate of Proposition 5.1 with $c=c_{7}$. By equation (6.4), we have

$$
\left\|\tilde{\nabla}_{A} \xi\right\|_{L^{p}}+\|\alpha\|_{L^{p}} \leq \delta \varepsilon^{2 / p-1 / 2} \leq \varepsilon^{2 / p-1}
$$


(provided that $\delta \leq 1$ ). Thus the hypotheses of Proposition 5.1 (i) and (ii) are satisfied with $\zeta=0$ and $\hat{\zeta}$ replaced by $\zeta$. Hence, by equation (6.11),

$$
\begin{aligned}
\left\|\tilde{\nabla}_{A} \xi\right\|_{L^{p}}+\|\alpha\|_{L^{p}} \leq & c_{6} c_{7}\|\xi\|_{L^{\infty}}\left(\varepsilon^{-1}\|\xi\|_{L^{p}}+\left\|\tilde{\nabla}_{A} \xi\right\|_{L^{p}}+\|\alpha\|_{L^{p}}\right) \\
& +c_{6} c_{7} \varepsilon\left(\|\alpha\|_{L^{\infty}}\|\alpha\|_{L^{p}}+\varepsilon^{-2}\|\xi\|_{L^{\infty}}\|\xi\|_{L^{p}}\right) \\
& +c_{6} \varepsilon\left\|F_{A}\right\|_{L^{p}}+c_{4}\|\xi\|_{L^{p}} \\
\leq & 3 c_{6} c_{7}\|\zeta\|_{\infty, \varepsilon}\left(\varepsilon^{-1}\|\xi\|_{L^{p}}+\left\|\tilde{\nabla}_{A} \xi\right\|_{L^{p}}+\|\alpha\|_{L^{p}}\right) \\
& +c_{6} \varepsilon\left\|F_{A}\right\|_{L^{p}}+c_{4}\|\xi\|_{L^{p}} \\
\leq & 3 c_{6} c_{7} \delta \varepsilon^{1 / 2}\left(\varepsilon^{-1}\|\xi\|_{L^{p}}+\left\|\tilde{\nabla}_{A} \xi\right\|_{L^{p}}+\|\alpha\|_{L^{p}}\right) \\
& +c_{0} c_{6} \varepsilon+c_{4}\|\xi\|_{L^{p}} \\
\leq & 3 c_{6} c_{7} \delta \varepsilon^{1 / 2}\left(\left\|\tilde{\nabla}_{A} \xi\right\|_{L^{p}}+\|\alpha\|_{L^{p}}\right) \\
& +c_{0} c_{6} \varepsilon+c_{4} \delta \varepsilon^{2 / p+1 / 2}+3 c_{6} c_{7} \delta^{2} \varepsilon^{2 / p} .
\end{aligned}
$$

Here the last two inequalities follow from (6.4). With $3 c_{6} c_{7} \delta \varepsilon^{1 / 2} \leq 1 / 2$, we have

$$
\left\|\tilde{\nabla}_{A} \xi\right\|_{L^{p}}+\|\alpha\|_{L^{p}} \leq c_{8}\left(\delta+\varepsilon^{1-2 / p}\right) \varepsilon^{2 / p},
$$

where $c_{8}:=2 c_{0} c_{6}+2 c_{4}+6 c_{6} c_{7}$. Since $\zeta$ satisfies equation (6.2), we can apply Lemma 4.8 (with $c=c_{5}$ ) to obtain

$$
\begin{aligned}
\|\zeta\|_{1, p, \varepsilon} & \leq c_{5}\left(\varepsilon\left\|\mathcal{D}^{\varepsilon} \zeta\right\|_{L^{p}}+\left\|\pi_{u} \mathcal{D}^{\varepsilon} \zeta\right\|_{L^{p}}\right) \\
& \leq c_{5}\left(2\left\|d \mathcal{F}_{1}^{\varepsilon}(0) \zeta\right\|_{L^{p}}+\varepsilon^{2}\left\|d \mathcal{F}_{3}^{\varepsilon}(0) \zeta\right\|_{L^{p}}\right) .
\end{aligned}
$$

By Proposition 5.1 (i) with $c=c_{7}$ and equation (6.12), we now have

$$
\begin{aligned}
\left\|d \mathcal{F}_{1}^{\varepsilon}(0) \zeta\right\|_{L^{p}}= & \left\|\mathcal{F}_{1}^{\varepsilon}(\zeta)-\mathcal{F}_{1}^{\varepsilon}(0)-d \mathcal{F}_{1}^{\varepsilon}(0) \zeta\right\|_{L^{p}} \\
\leq & c_{7}\|\xi\|_{L^{\infty}}\left(\|\xi\|_{L^{p}}+\left\|\tilde{\nabla}_{A} \xi\right\|_{L^{p}}+\|\alpha\|_{L^{p}}\right) \\
& +c_{7}\|\xi\|_{L^{\infty}}^{2}\left(\left\|\tilde{\nabla}_{A} \xi\right\|_{L^{p}}+\|\alpha\|_{L^{p}}\right) \\
\leq & 2 c_{7}\|\xi\|_{L^{\infty}}\left(\|\xi\|_{L^{p}}+\left\|\tilde{\nabla}_{A} \xi\right\|_{L^{p}}+\|\alpha\|_{L^{p}}\right) \\
\leq & 2 c_{7}\|\zeta\|_{\infty, \varepsilon}\left(\delta \varepsilon^{2 / p+1 / 2}+c_{8}\left(\delta+\varepsilon^{1-2 / p}\right) \varepsilon^{2 / p}\right) \\
\leq & c_{9}\left(\delta+\varepsilon^{1-2 / p}\right)\|\zeta\|_{1, p, \varepsilon} .
\end{aligned}
$$

Here we have used the fact that $\|\xi\|_{L^{\infty}} \leq \delta \varepsilon^{1 / 2} \leq 1$. Moreover, the penultimate inequality follows from equations (6.4) and (6.12) and the last inequality, with a suitable constant $c_{9}=c_{9}\left(p, c_{0}\right)$, follows from Lemma 4.4. 
By Proposition 5.1 (ii) with $c=c_{7}$, we have

$$
\begin{aligned}
\left\|d \mathcal{F}_{3}^{\varepsilon}(0) \zeta\right\|_{L^{p}} & \leq\left\|\mathcal{F}_{3}^{\varepsilon}(0)-\mathcal{F}_{3}^{\varepsilon}(\zeta)-d \mathcal{F}_{3}^{\varepsilon}(0) \zeta\right\|_{L^{p}}+\left\|F_{A}\right\|_{L^{p}} \\
& \leq c_{7}\left(\|\alpha\|_{L^{\infty}}\|\alpha\|_{L^{p}}+\varepsilon^{-2}\|\xi\|_{L^{\infty}}\|\xi\|_{L^{p}}\right)+\left\|F_{A}\right\|_{L^{p}} \\
& \leq 2 c_{7}\left(\varepsilon^{-1}\|\alpha\|_{L^{p}}+\varepsilon^{-2}\|\xi\|_{L^{p}}\right)\|\zeta\|_{\infty, \varepsilon}+\left\|F_{A}\right\|_{L^{p}} \\
& \leq 2 c_{7} \delta \varepsilon^{2 / p} \varepsilon^{-3 / 2}\|\zeta\|_{\infty, \varepsilon}+\left\|F_{A}\right\|_{L^{p}} \\
& \leq c_{10} \delta \varepsilon^{-3 / 2}\|\zeta\|_{1, p, \varepsilon}+\left\|F_{A}\right\|_{L^{p}} .
\end{aligned}
$$

Here the penultimate inequality follows from equation (6.4) and the last follows from Lemma 4.4. Combining these two estimates with equation (6.13), we obtain

$$
\|\zeta\|_{1, p, \varepsilon} \leq c_{5}\left(2 c_{9}\left(\delta+\varepsilon^{1-2 / p}\right)+c_{10} \delta \varepsilon^{1 / 2}\right)\|\zeta\|_{1, p, \varepsilon}+c_{5} \varepsilon^{2}\left\|F_{A}\right\|_{L^{p}}
$$

If $\delta$ and $\varepsilon$ are sufficiently small, we obtain

$$
\|\zeta\|_{1, p, \varepsilon} \leq 2 c_{5} \varepsilon^{2}\left\|F_{A}\right\|_{L^{p}} \leq 2 c_{0} c_{5} \varepsilon^{2} .
$$

Hence the result follows from the uniqueness argument at the end of the proof of Theorem 6.1.

Corollary 6.3 has a slightly stronger hypothesis than Theorem 6.2 , however, it does not seem to have a simpler proof. In order to significantly simplify the proof we would have to further strengthen the hypothesis and assume $\|\zeta\|_{1, p, \varepsilon} \leq \delta \varepsilon^{2 / p+1}$ with a small constant $\delta$ (instead of $\|\zeta\|_{1, p, \varepsilon} \leq$ $\delta \varepsilon^{2 / p+1 / 2}$ as in Corollary 6.3). Under this hypothesis, uniqueness can be established with the same straight forward argument that is used at the end of the proof of Theorem 6.1. However, such a weaker result just fails to suffice for the proof of Theorem 3.3. Namely, in Section 10 we shall establish an inequality of the form

$$
\|\zeta\|_{1, p, \varepsilon} \leq c \varepsilon^{2 / p+1}
$$

under the hypotheses of Theorem 3.3. In this inequality, the constant $c$ is not small and so the argument in the proof of Theorem 6.1 does not suffice to give uniqueness. However, if $\varepsilon$ is chosen so small that $c \varepsilon^{1 / 2} \leq \delta$ then we can use Corollary 6.3 to obtain uniqueness.

\section{Relative Coulomb gauge}

This section is of preparatory nature. We prove a local slice theorem for the action of the gauge group $\mathcal{G}=\mathcal{G}(P)$ on $\mathcal{B}=C_{\mathrm{G}}^{\infty}(P, M) \times \mathcal{A}(P)$. The infinitesimal action is the operator $d_{(u, A)}: \Omega^{0}\left(\Sigma, \mathfrak{g}_{P}\right) \rightarrow T_{(u, A)} \mathcal{B}$ given by

$$
d_{(u, A)} \eta:=\left(-L_{u} \eta, d_{A} \eta\right)
$$


Denote by $d_{(u, A)}^{*_{\varepsilon}}$ its formal adjoint with respect to the $\varepsilon$-inner product, i.e.,

$$
d_{(u, A)}^{*_{\varepsilon}}(\xi, \alpha):=\varepsilon^{2} d_{A}^{*} \alpha-L_{u}^{*} \xi
$$

for $(\xi, \alpha) \in T_{(u, A)} \mathcal{B}$. The next proposition restates the local slice theorem for the $\mathcal{G}$-action on $\mathcal{B}$ with $\varepsilon$-dependent norms for elements $\left(u_{0}, A_{0}\right)$ of the moduli space $\widetilde{\mathcal{M}}_{B, \Sigma}^{0}$. The result continues to hold for every element $\left(u_{0}, A_{0}\right) \in \mathcal{B}$ with $\mu\left(u_{0}\right)=0$. However, in this generality, more care must be taken in determining the norm on $\mathcal{B}$ with respect to which the constants $c$ and $\delta$ depend continuously on $\left(u_{0}, A_{0}\right)$. In the case of $J$-holomorphic curves, the $W^{1, p}$-norm controls all higher derivatives and therefore the choice of the norm is immaterial.

Proposition 7.1. Assume $(H 1)$. For every $p>2$ and every $c_{0}>0$, there exist positive constants $\delta$ and $c$ such that the following holds for every $\varepsilon \in$ $(0,1]$. Let $\left(u_{0}, A_{0}\right) \in \widetilde{\mathcal{M}}_{B, \Sigma}^{0}\left(c_{0}\right)$ and $\zeta=(\xi, \alpha) \in T_{\left(u_{0}, A_{0}\right)} \mathcal{B}$ such that

$$
\|\zeta\|_{1, p, \varepsilon ;\left(u_{0}, A_{0}\right)} \leq \delta \varepsilon^{2 / p} .
$$

Denote $(u, A):=\left(\exp _{u_{0}}(\xi), A_{0}+\alpha\right)$. Then there exist a unique pair $\zeta_{0}=$ $\left(\xi_{0}, \alpha_{0}\right) \in T_{\left(u_{0}, A_{0}\right)} \mathcal{B}$ and a unique section $\eta_{0} \in \Omega^{0}\left(\Sigma, \mathfrak{g}_{P}\right)$ such that

$$
\begin{gathered}
d_{\left(u_{0}, A_{0}\right)}^{*_{\varepsilon}} \zeta_{0}=0, \quad g^{*}(u, A)=\left(\exp _{u_{0}}\left(\xi_{0}\right), A_{0}+\alpha_{0}\right), \quad g:=e^{\eta_{0}}, \\
\left\|\eta_{0}\right\|_{2, p, \varepsilon ; A_{0}}+\left\|\zeta_{0}\right\|_{1, p, \varepsilon ;\left(u_{0}, A_{0}\right)} \leq c\|\zeta\|_{1, p, \varepsilon ;\left(u_{0}, A_{0}\right)} .
\end{gathered}
$$

Lemma 7.2 (Linear estimate). For every $p \geq 2$ and every $c_{0}>0$, there is a constant $c>0$ such that the following holds for every $\left(u_{0}, A_{0}\right) \in \widetilde{\mathcal{M}}_{B, \Sigma}^{0}\left(c_{0}\right)$ and every $\varepsilon \in(0,1]$. If $\zeta=(\xi, \alpha) \in W^{1, p}\left(\Sigma, u_{0}^{*} T M / \mathrm{G}\right) \oplus\left(T^{*} \Sigma \otimes \mathfrak{g}_{P}\right)$ then there is a unique $\eta \in W^{2, p}\left(\Sigma, \mathfrak{g}_{P}\right)$ such that

$$
d_{\left(u_{0}, A_{0}\right)}^{*_{\varepsilon}} d_{\left(u_{0}, A_{0}\right)} \eta=d_{\left(u_{0}, A_{0}\right)}^{*_{\varepsilon}} \zeta .
$$

Moreover, $\eta$ satisfies the estimates

$$
\|\eta\|_{1, p, \varepsilon} \leq c\|\zeta\|_{0, p, \varepsilon}, \quad\|\eta\|_{2, p, \varepsilon} \leq c\left\|d_{\left(u_{0}, A_{0}\right)}^{*_{\varepsilon}} \zeta\right\|_{L^{p}} .
$$

Lemma 7.3 (Quadratic estimate). For every $p>2$ and every $c_{0}>0$, there exist positive constants $\delta$ and $c$ such that the following holds for every $\left(u_{0}, A_{0}\right) \in \widetilde{\mathcal{M}}_{B, \Sigma}^{0}\left(c_{0}\right)$ and every $\varepsilon \in(0,1]$. Assume that $\zeta_{0}=\left(\xi_{0}, \alpha_{0}\right) \in$ $T_{\left(u_{0}, A_{0}\right)} \mathcal{B}$ and $\eta \in \Omega^{0}\left(\Sigma, \mathfrak{g}_{P}\right)$ satisfy

$$
\|\eta\|_{2, p, \varepsilon}+\left\|\zeta_{0}\right\|_{1, p, \varepsilon} \leq \delta \varepsilon^{2 / p}
$$

Then there exists a unique pair $\zeta_{1}=\left(\xi_{1}, \alpha_{1}\right) \in T_{\left(u_{0}, A_{0}\right)} \mathcal{B}$ such that

$$
\begin{aligned}
& \left(\exp _{u_{0}}\left(\xi_{1}\right), A_{0}+\alpha_{1}\right)=g^{*}\left(\exp _{u_{0}}\left(\xi_{0}\right), A_{0}+\alpha_{0}\right), \quad g:=e^{\eta}, \\
& \left\|\zeta_{1}-\zeta_{0}\right\|_{0, p, \varepsilon} \leq c\|\eta\|_{1, p, \varepsilon}, \quad\left\|\zeta_{1}-\zeta_{0}\right\|_{1, p, \varepsilon} \leq c\|\eta\|_{2, p, \varepsilon} .
\end{aligned}
$$


Moreover,

$$
\left\|d_{\left(u_{0}, A_{0}\right)}^{*_{\varepsilon}}\left(\zeta_{1}-\zeta_{0}-d_{\left(u_{0}, A_{0}\right)} \eta\right)\right\|_{L^{p}} \leq c \varepsilon^{-2 / p}\left(\left\|\zeta_{0}\right\|_{1, p, \varepsilon}+\|\eta\|_{2, p, \varepsilon}\right)\|\eta\|_{1, p, \varepsilon} .
$$

Lemma 7.4. For every $p \geq 2$ and every $c_{0}>0$, there exist positive constants $\delta$ and $c$ such that the following holds for every $\left(u_{0}, A_{0}\right) \in \widetilde{\mathcal{M}}_{B, \Sigma}^{0}\left(c_{0}\right)$ and every $\varepsilon \in(0,1]$. If $\eta_{1}, \eta_{2} \in \Omega^{0}\left(\Sigma, \mathfrak{g}_{P}\right)$ satisfy $\left\|\eta_{1}\right\|_{L^{\infty}} \leq \delta$ and $\left\|\eta_{2}\right\|_{L^{\infty}} \leq \delta$ then there exists a unique element $\eta \in \Omega^{0}\left(\Sigma, \mathfrak{g}_{P}\right)$ such that

$$
e^{\eta}=e^{\eta_{1}} e^{\eta_{2}}, \quad 2^{-1}\|\eta\|_{L^{\infty}} \leq\left\|\eta_{1}+\eta_{2}\right\|_{L^{\infty}} \leq 2\|\eta\|_{L^{\infty}} .
$$

Moreover, $\eta$ satisfies the estimate

$$
c^{-1}\|\eta\|_{2, p, \varepsilon ; A_{0}} \leq\left\|\eta_{1}+\eta_{2}\right\|_{2, p, \varepsilon ; A_{0}} \leq c\|\eta\|_{2, p, \varepsilon ; A_{0}} .
$$

Proof. For a fixed connection $A_{0}$ and $\varepsilon=1$, the result is obvious. Choose $c_{1}, c_{2}$, and $c_{3}$ such that

$$
c_{k}^{-1}\|\eta\|_{W^{k, p}} \leq\left\|\eta_{1}+\eta_{2}\right\|_{W^{k, p}} \leq c_{k}\|\eta\|_{W^{k, p}}
$$

for $k=1,2,3$, whenever $\eta_{1}, \eta_{2}$, and $\eta$ are sufficiently small in the $C^{0}$-norm and satisfy $e^{\eta}=e^{\eta_{1}} e^{\eta_{2}}$. Here the $W^{k, p}$ norms are understood with respect to the connection $A_{0}$. It follows that

$$
\varepsilon\left\|d_{A_{0}}\left(\eta_{1}+\eta_{2}\right)\right\|_{L^{p}} \leq \varepsilon c_{1}\left(\left\|d_{A_{0}} \eta\right\|_{L^{p}}+\|\eta\|_{L^{p}}\right) \leq c_{1}\|\eta\|_{1, p, \varepsilon ; A_{0}},
$$

and hence

$$
\left\|\eta_{1}+\eta_{2}\right\|_{1, p, \varepsilon ; A_{0}} \leq\left(c_{0}+c_{1}\right)\|\eta\|_{1, p, \varepsilon ; A_{0}}
$$

for $0 \leq \varepsilon \leq 1$. The other three inequalities follow by similar arguments. This proves the lemma for a fixed connection $A_{0}$. The constant $c$ depends continuously on $A_{0}$ with respect to the $C^{1}$-norm, and is gauge-invariant (with respect to the action of $\mathcal{G}$ on $\Omega^{0}\left(\Sigma, \mathfrak{g}_{P}\right)$ by conjugation). Hence, by Lemma 2.2 , it can be chosen independent of $A_{0}$ as long as $\left(u_{0}, A_{0}\right) \in$ $\widetilde{\mathcal{M}}_{B, \Sigma}^{0}\left(c_{0}\right)$ for some $u_{0}$.

Proof of Lemma 7.2. The operator $d_{\left(u_{0}, A_{0}\right)}^{*_{\varepsilon}} d_{\left(u_{0}, A_{0}\right)}: W^{2, p}\left(\Sigma, \mathfrak{g}_{P}\right) \rightarrow L^{p}$ $\left(\Sigma, \mathfrak{g}_{P}\right)$ is given by

$$
d_{\left(u_{0}, A_{0}\right)}^{*_{\varepsilon}} d_{\left(u_{0}, A_{0}\right)} \eta=\varepsilon^{2} d_{A_{0}}^{*} d_{A_{0}} \eta+L_{u_{0}}^{*} L_{u_{0}} \eta .
$$

By our standing hypotheses, $\mu^{-1}(0)$ is compact and $L_{x}: \mathfrak{g} \rightarrow T_{x} M$ is injective for every $x \in \mu^{-1}(0)$. Hence there exists a constant $c_{1}>0$ such that

$$
c_{1}^{-1}|\eta| \leq\left|L_{x} \eta\right|_{z} \leq c_{1}|\eta|
$$

for every $x \in \mu^{-1}(0)$, every $\eta \in \mathfrak{g}$, and every $z \in \Sigma$. (Here $|\cdot|_{z}$ denotes the metric on $M$ induced by $J_{z}$ and $\omega$.) Hence the operator $d_{\left(u_{0}, A_{0}\right)}^{*_{\varepsilon}} d_{\left(u_{0}, A_{0}\right)}$ is injective and hence, by elliptic regularity, it is bijective. 
Next we prove that there exists a constant $c_{2}=c_{2}\left(p, c_{0}\right)>0$ such that, for every pair $\left(u_{0}, A_{0}\right) \in \widetilde{\mathcal{M}}_{B, \Sigma}^{0}\left(c_{0}\right)$, every $\eta \in \Omega^{0}\left(\Sigma, \mathfrak{g}_{P}\right)$, and every $\varepsilon \in(0,1]$, we have

$$
\left\|d_{A_{0}} \eta\right\|_{L^{p}} \leq \varepsilon\left\|d_{A_{0}}^{*} d_{A_{0}} \eta\right\|_{L^{p}}+c_{2} \varepsilon^{-1}\|\eta\|_{L^{p}} .
$$

For a fixed connection $A_{0} \in \mathcal{A}(P)$ this follows directly from the interpolation inequality in [8, Theorem 7.27] and the $L^{p}$-estimate for the operator $d_{A_{0}}^{*} d_{A_{0}}$. Now the identity

$$
\begin{aligned}
d_{A}^{*} d_{A} \eta-d_{A_{0}}^{*} d_{A_{0}} \eta= & {\left[A-A_{0} \wedge d_{A_{0}} \eta\right]+*\left[*\left(A-A_{0}\right) \wedge d_{A_{0}} \eta\right] } \\
& -*\left[d_{A_{0}} *\left(A-A_{0}\right), \eta\right]+*\left[*\left(A-A_{0}\right) \wedge\left[A-A_{0}, \eta\right]\right]
\end{aligned}
$$

shows that the constant in equation (7.10) depends continuously on $A$ with respect to the $C^{1}$-norm. Moreover, the inequality (7.10) is gauge-invariant. Hence it follows from Lemma 2.2 (with $\ell=2$ ) and the Arzéla-Ascoli theorem that equation (7.10) holds with a uniform constant $c_{2}$ for all $\left(u_{0}, A_{0}\right) \in$ $\widetilde{\mathcal{M}}_{B, \Sigma}^{0}\left(c_{0}\right)$.

Using the identity

$$
d|\eta|^{p-2}=(p-2)|\eta|^{p-4}\left\langle\eta, d_{A_{0}} \eta\right\rangle \in \Omega^{1}(\Sigma)
$$

for $\eta \in \Omega^{0}\left(\Sigma, \mathfrak{g}_{P}\right)$ and integration by parts, we obtain

$$
\int_{\Sigma}|\eta|^{p-2}\left|d_{A_{0}} \eta\right|^{2}=\int_{\Sigma}|\eta|^{p-2}\left\langle\eta, d_{A_{0}}^{*} d_{A_{0}} \eta\right\rangle-(p-2) \int_{\Sigma}|\eta|^{p-4}\left|\left\langle\eta, d_{A_{0}} \eta\right\rangle\right|^{2} .
$$

The last term on the right is negative. Now equation (7.3) is equivalent to

$$
\varepsilon^{2} d_{A_{0}}^{*} \alpha-L_{u_{0}}^{*} \xi=\varepsilon^{2} d_{A_{0}}^{*} d_{A_{0}} \eta+L_{u_{0}}^{*} L_{u_{0}} \eta
$$

Hence, by the previous identity and equation (7.9), we have

$$
\begin{aligned}
\int_{\Sigma} & \left(c_{1}^{-2}|\eta|^{p}+\varepsilon^{2}|\eta|^{p-2}\left|d_{A_{0}} \eta\right|^{2}\right) \\
\quad \leq & \int_{\Sigma}|\eta|^{p-2}\left(\left|L_{u_{0}} \eta\right|^{2}+\varepsilon^{2}\left|d_{A_{0}} \eta\right|^{2}\right) \\
\quad \leq & \int_{\Sigma}|\eta|^{p-2}\left\langle\eta, \varepsilon^{2} d_{A_{0}}^{*} d_{A_{0}} \eta+L_{u_{0}}^{*} L_{u_{0}} \eta\right\rangle \\
7.11) & \int_{\Sigma}|\eta|^{p-2}\left\langle\eta, \varepsilon^{2} d_{A_{0}}^{*} \alpha-L_{u_{0}}^{*} \xi\right\rangle \\
\quad= & \int_{\Sigma}|\eta|^{p-2}\left(\varepsilon^{2}\left\langle d_{A_{0}} \eta, \alpha\right\rangle+\varepsilon^{2}(p-2)|\eta|^{-2}\left\langle\left\langle\eta, d_{A_{0}} \eta\right\rangle,\langle\eta, \alpha\rangle\right\rangle-\left\langle L_{u_{0}} \eta, \xi\right\rangle\right) \\
\leq & c_{1} \int_{\Sigma}|\eta|^{p-1}|\xi|+\varepsilon^{2}(p-1) \int_{\Sigma}|\eta|^{p-2}\left|d_{A_{0}} \eta\right||\alpha| \\
\leq & c_{1} \int_{\Sigma}|\eta|^{p-1}|\xi|+\frac{\varepsilon^{2}(p-1)^{2}}{2} \int_{\Sigma}|\eta|^{p-2}|\alpha|^{2}+\frac{\varepsilon^{2}}{2} \int_{\Sigma}|\eta|^{p-2}\left|d_{A_{0}} \eta\right|^{2} .
\end{aligned}
$$


Therefore, by Hölder's inequality,

$$
\begin{aligned}
& c_{1}^{-2}\|\eta\|_{L^{p}}^{p} \leq c_{1}\|\eta\|_{L^{p}}^{p-1}\|\xi\|_{L^{p}}+\frac{\varepsilon^{2}(p-1)^{2}}{2}\|\eta\|_{L^{p}}^{p-2}\|\alpha\|_{L^{p}}^{2}, \\
& c_{1}^{-2}\|\eta\|_{L^{p}}^{p} \leq\|\eta\|_{L^{p}}^{p-1}\|\tilde{\eta}\|_{L^{p}}, \quad \tilde{\eta}:=\varepsilon^{2} d_{A_{0}}^{*} d_{A_{0}} \eta+L_{u_{0}}^{*} L_{u_{0}} \eta .
\end{aligned}
$$

The last inequality follows from the third line in equation (7.11). Hence

$$
c_{1}^{-2}\|\eta\|_{L^{p}}^{2} \leq c_{1}\|\eta\|_{L^{p}}\|\xi\|_{L^{p}}+\frac{\varepsilon^{2}(p-1)^{2}}{2}\|\alpha\|_{L^{p}}^{2}
$$

and hence, using $c_{1}^{3}\|\eta\|_{L^{p}}\|\xi\|_{L^{p}} \leq 1 / 2\|\eta\|_{L^{p}}^{2}+1 / 2 c_{1}^{6}\|\xi\|_{L^{p}}^{2}$ we obtain

$$
\|\eta\|_{L^{p}}^{2} \leq c_{1}^{6}\|\xi\|_{L^{p}}^{2}+c_{1}^{2} \varepsilon^{2}(p-1)^{2}\|\alpha\|_{L^{p}}^{2} .
$$

Thus we have proved the inequalities

$$
\|\eta\|_{L^{p}} \leq c_{1} \max \left\{p-1, c_{1}^{2}\right\}\|\zeta\|_{0, p, \varepsilon}, \quad\|\eta\|_{L^{p}} \leq c_{1}^{2}\|\tilde{\eta}\|_{L^{p}} .
$$

By equations (7.9), (7.10), and (7.12),

$$
\begin{aligned}
\|\eta\|_{2, p, \varepsilon ; A_{0}} & =\|\eta\|_{L^{p}}+\varepsilon\left\|d_{A_{0}} \eta\right\|_{L^{p}}+\varepsilon^{2}\left\|d_{A_{0}}^{*} d_{A_{0}} \eta\right\|_{L^{p}} \\
& \leq\left(1+c_{2}\right)\|\eta\|_{L^{p}}+2 \varepsilon^{2}\left\|d_{A_{0}}^{*} d_{A_{0}} \eta\right\|_{L^{p}} \\
& \leq\left(1+c_{2}+2 c_{1}^{2}\right)\|\eta\|_{L^{p}}+2\|\tilde{\eta}\|_{L^{p}} \\
& \leq\left(2+c_{1}^{2}\left(1+c_{2}+2 c_{1}^{2}\right)\right)\|\tilde{\eta}\|_{L^{p}} .
\end{aligned}
$$

This proves the second estimate in equations (7.4).

To prove the first estimate in euations (7.4), we use a rescaling argument in local holomorphic coordinates on $\Sigma$. Cover $\Sigma$ by finitely many open sets, each of which is holomorphically diffeomorphic to the unit square in $\mathbb{C}$, suppose that the coordinate charts extend to a closed square of side length two, and choose trivializations of the bundle $P$ over each of these (extended) open sets. In these coordinates, we write the metric in the form $\lambda^{2}\left(d s^{2}+d t^{2}\right)$, and we write $A:=A_{0}=\Phi d s+\Psi d t, \alpha=\varphi d s+\psi d t$. Moreover, $u:=u_{0}:[0,2]^{2} \rightarrow M, \xi:[0,2]^{2} \rightarrow T M$ is a vector field along $u$, and $\eta:[0,2]^{2} \rightarrow \mathfrak{g}$. In this notation, equation (7.3) has the form

$$
\nabla_{s} \nabla_{s} \eta+\nabla_{t} \nabla_{t} \eta=\nabla_{s} \varphi+\nabla_{t} \psi+\left(\frac{\lambda}{\varepsilon}\right)^{2} L_{u}^{*}\left(L_{u} \eta+\xi\right)
$$

where $\nabla_{s} \eta:=\nabla_{A, s}^{\mathrm{G}} \eta=\partial_{s} \eta+[\Phi, \eta]$ and $\nabla_{t} \eta:=\nabla_{A, t}^{\mathrm{G}} \eta=\partial_{t} \eta+[\Psi, \eta]$. Now we introduce new functions, defined on the square $[0,2 / \varepsilon]^{2}$, by

$$
\begin{aligned}
\tilde{\eta}(s, t) & :=\eta(\varepsilon s, \varepsilon t), & \tilde{\lambda}(s, t) & :=\lambda(\varepsilon s, \varepsilon t), \\
\tilde{\xi}(s, t) & :=\xi(\varepsilon s, \varepsilon t), & \tilde{u}(s, t) & :=u(\varepsilon s, \varepsilon t), \\
\tilde{\varphi}(s, t) & :=\varepsilon \varphi(\varepsilon s, \varepsilon t), & \tilde{\Phi}(s, t) & :=\varepsilon \Phi(\varepsilon s, \varepsilon t), \\
\tilde{\psi}(s, t) & :=\varepsilon \psi(\varepsilon s, \varepsilon t), & \tilde{\Psi}(s, t) & :=\varepsilon \Psi(\varepsilon s, \varepsilon t) .
\end{aligned}
$$


Then equation (7.13) is equivalent to

$$
\tilde{\nabla}_{s} \tilde{\nabla}_{s} \tilde{\eta}+\tilde{\nabla}_{t} \tilde{\nabla}_{t} \tilde{\eta}=\tilde{\nabla}_{s} \tilde{\varphi}+\tilde{\nabla}_{t} \tilde{\psi}+\tilde{\lambda}^{2} L_{\tilde{u}}^{*}\left(L_{\tilde{u}} \tilde{\eta}+\tilde{\xi}\right)
$$

where $\tilde{\nabla}_{s} \tilde{\eta}:=\partial_{s} \tilde{\eta}+[\tilde{\Phi}, \tilde{\eta}]$ and $\tilde{\nabla}_{t} \tilde{\eta}:=\partial_{t} \tilde{\eta}+[\tilde{\Psi}, \tilde{\eta}]$. With $\Delta:=\partial_{s} \partial_{s}+\partial_{t} \partial_{t}$, this equation can be written in the form

$$
\begin{gathered}
\Delta \tilde{\eta}=\partial_{s} \tilde{f}+\partial_{t} \tilde{g}+\tilde{h}, \quad \tilde{f}:=\tilde{\varphi}-2[\tilde{\Phi}, \tilde{\eta}], \quad \tilde{g}:=\tilde{\psi}-2[\tilde{\Psi}, \tilde{\eta}] \\
\tilde{h}:=\tilde{\lambda}^{2} L_{\tilde{u}}^{*}\left(L_{\tilde{u}} \tilde{\eta}+\tilde{\xi}\right)+[\tilde{\Phi}, \tilde{\varphi}-[\tilde{\Phi}, \tilde{\eta}]]+[\tilde{\Psi}, \tilde{\psi}-[\tilde{\Psi}, \tilde{\eta}]]+\left[\partial_{s} \tilde{\Phi}+\partial_{t} \tilde{\Psi}, \tilde{\eta}\right] .
\end{gathered}
$$

Hence there exists a constant $c_{3}>0$ such that, for all real numbers $a, b$ such that $1 / 2 \leq a<b \leq 2 / \varepsilon-1 / 2$, we have

$$
\int_{[a, b]^{2}}\left(\left|\tilde{\nabla}_{s} \tilde{\eta}\right|^{p}+\left|\tilde{\nabla}_{t} \tilde{\eta}\right|^{p}\right) \leq c_{3} \int_{[a-1 / 2, b+1 / 2]^{2}}\left(|\tilde{f}|^{p}+|\tilde{g}|^{p}+|\tilde{h}|^{p}+|\tilde{\eta}|^{p}\right) .
$$

Here the constant $c_{3}$ is independent of $a$ and $b$. It follows that

$$
\begin{aligned}
\int_{[a, b]^{2}}\left(\left|\tilde{\nabla}_{s} \tilde{\eta}\right|^{p}+\left|\tilde{\nabla}_{t} \tilde{\eta}\right|^{p}\right) \tilde{\lambda}^{2-p} \leq & c_{4} \int_{[a-1 / 2, b+1 / 2]^{2}}\left(|\tilde{\varphi}|^{p}+|\tilde{\psi}|^{p}\right) \tilde{\lambda}^{2-p} \\
& +c_{4} \int_{[a-1 / 2, b+1 / 2]^{2}}\left(|\tilde{\xi}|^{p}+|\tilde{\eta}|^{p}\right) \tilde{\lambda}^{2},
\end{aligned}
$$

where the constant $c_{4}$ depends on the metric and on the $C^{1}$-norms of $\tilde{\Phi}$ and $\tilde{\Psi}$. With $a=1 / 2 \varepsilon, b=3 / 2 \varepsilon$, and $0<\varepsilon \leq 1$ we obtain

$$
\begin{aligned}
\varepsilon^{p} \int_{[1 / 2,3 / 2]^{2}}\left(\left|\nabla_{s} \eta\right|^{p}+\left|\nabla_{t} \eta\right|^{p}\right) \lambda^{2-p} \leq & c_{4} \varepsilon^{p} \int_{[0,2]^{2}}\left(|\varphi|^{p}+|\psi|^{p}\right) \lambda^{2-p} \\
& +c_{4} \int_{[0,2]^{2}}\left(|\xi|^{p}+|\eta|^{p}\right) \lambda^{2} .
\end{aligned}
$$

Hence, by taking the sum over the coordinate charts,

$$
\varepsilon\left\|d_{A_{0}} \eta\right\|_{L^{p}} \leq N^{1 / p} c_{4}\left(\varepsilon\|\alpha\|_{L^{p}}+\|\xi\|_{L^{p}}+\|\eta\|_{L^{p}}\right) .
$$

Here $N$ is the number of open sets in the cover and the constant $c_{4}$ depends continuously on $A_{0}$ with respect to the $C^{1}$-norm. Hence, by Lemma 2.2, $c_{4}$ can be chosen independent of the pair $\left(u_{0}, A_{0}\right) \in \widetilde{\mathcal{M}}_{B, \Sigma}^{0}\left(c_{0}\right)$. Combining the last inequality with equations (7.12), we obtain the first estimate in equations (7.4) as claimed.

In the following proof, we use the identity

$$
\left(e^{\eta}\right)^{*} A-A-d_{A} \eta=\sum_{k=1}^{\infty} \frac{(-1)^{k}}{(k+1) !} \operatorname{ad}(\eta)^{k} d_{A} \eta
$$

for $A \in \mathcal{A}(P)$ and $\eta \in \Omega^{0}\left(\Sigma, \mathfrak{g}_{P}\right)$, where $\operatorname{ad}(\eta) \alpha:=[\eta, \alpha]$ for $\alpha \in \Omega^{1}\left(\Sigma, \mathfrak{g}_{P}\right)$. 
Proof of Lemma 7.3. We denote by $c_{1}, c_{2}, c_{3}, \ldots$ positive constants depending only on $p$ and $c_{0}$. Fix a pair $\left(u_{0}, A_{0}\right) \in \widetilde{\mathcal{M}}_{B, \Sigma}^{0}\left(c_{0}\right)$ and choose a positive constant $\delta_{0}$ that is smaller than the injectivity radius of $M$ on the compact set $u_{0}(P)$. Suppose that $\eta \in \Omega^{0}\left(\Sigma, \mathfrak{g}_{P}\right)$ and $\zeta_{0}=\left(\xi_{0}, \alpha_{0}\right) \in T_{\left(u_{0}, A_{0}\right)} \mathcal{B}$ satisfy the hypotheses of Lemma 7.3 with a sufficiently small constant $\delta>0$. Let $c_{1}$ be the constant of Lemma 4.4. Then, by equation (7.5),

$$
\left\|\xi_{0}\right\|_{L^{\infty}}+\|\eta\|_{L^{\infty}} \leq c_{1} \varepsilon^{-2 / p}\left(\left\|\xi_{0}\right\|_{1, p, \varepsilon}+\|\eta\|_{2, p, \varepsilon}\right) \leq c_{1} \delta .
$$

If $\delta$ is sufficiently small, it follows that the $C^{0}$-distance between $e^{-r \eta} \exp _{u_{0}}$ $\left(\xi_{0}\right)$ and $u_{0}$ is smaller than $\delta_{0}$ for every $r \in[0,1]$. Hence there exists a unique smooth path $[0,1] \rightarrow T_{\left(u_{0}, A_{0}\right)} \mathcal{B}: r \mapsto \zeta_{r}=\left(\xi_{r}, \alpha_{r}\right)$ starting at $\zeta_{0}$ such that

$$
\left(\exp _{u_{0}}\left(\xi_{r}\right), A_{0}+\alpha_{r}\right)=g^{*}(u, A)
$$

where

$$
(u, A):=\left(\exp _{u_{0}}\left(\xi_{0}\right), A_{0}+\alpha_{0}\right), \quad g:=e^{r \eta} .
$$

The endpoint $\zeta_{1}$ of this path obviously satisfies equation (7.6). We prove the inequalities

$$
\left\|d_{\left(u_{0}, A_{0}\right)}^{*_{\varepsilon}}\left(\partial_{r} \zeta_{r}-d_{\left(u_{0}, A_{0}\right)} \eta\right)\right\|_{L^{p}} \leq c \varepsilon^{-2 / p}\left(\left\|\zeta_{0}\right\|_{1, p, \varepsilon}+\|\eta\|_{2, p, \varepsilon}\right)\|\eta\|_{1, p, \varepsilon}
$$

for $0 \leq r \leq 1$, where the constant $c$ depends only on $c_{0}$ and $p$. Then the inequalities (7.7) and (7.8) follow by integrating the function $r \mapsto \partial_{r} \zeta_{r}$ over the interval $0 \leq r \leq 1$.

For every $u \in C_{\mathrm{G}}^{\infty}(P, M)$ whose $C^{0}$-distance to $u_{0}$ is less than $\delta_{0}$, we define the linear operator $Z(u): \Omega^{0}\left(\Sigma, \mathfrak{g}_{P}\right) \rightarrow \Omega^{0}\left(\Sigma, u_{0}^{*} T M / \mathrm{G}\right)$ by

$$
Z(u) \hat{\eta}:=d \exp _{u_{0}}^{-1}(u) L_{u} \hat{\eta}
$$

for $\hat{\eta} \in \Omega^{0}\left(\Sigma, \mathfrak{g}_{P}\right)$. Then

$$
\partial_{r} \zeta_{r}=\left(-Z\left(g^{-1} u\right) \eta, d_{g^{*} A} \eta_{0}\right),
$$

where $(u, A)$ and $g$ are as in equations (7.15). We prove the first inequality in equation (7.16). Since $A=A_{0}+\alpha_{0}$ we have

$$
d_{g^{*} A} \eta=d_{A_{0}} \eta+\left[g^{*} A_{0}-A_{0}, \eta\right]+\left[g^{-1} \alpha_{0} g, \eta\right],
$$

and we must estimate the three terms on the right with $g:=e^{r \eta}$. Since $\|\eta\|_{L^{\infty}} \leq c_{1} \delta$ it follows from equation (7.14), with $\eta$ replaced by $r \eta$, that

$$
\left\|\left[g^{*} A_{0}-A_{0}, \eta\right]\right\|_{L^{p}} \leq c_{2}\left\|d_{A_{0}} \eta\right\|_{L^{p}}\|\eta\|_{L^{\infty}} \leq c_{3} \varepsilon^{-1-2 / p}\|\eta\|_{1, p, \varepsilon}^{2} .
$$

Moreover,

$$
\left\|\left[g^{-1} \alpha_{0} g, \eta\right]\right\|_{L^{p}} \leq \varepsilon^{-1-2 / p}\left\|\zeta_{0}\right\|_{0, p, \varepsilon}\|\eta\|_{1, p, \varepsilon} .
$$


Hence, by equations (7.5) and (7.19), $\left\|d_{g^{*} A} \eta\right\|_{L^{p}} \leq c_{4} \varepsilon^{-1}\|\eta\|_{1, p, \varepsilon}$ and hence the first inequality in equation (7.17) follows from equation (7.18).

Next we prove the second inequality in equations (7.16) and (7.17). Using the identity

$$
\begin{aligned}
d_{A_{0}}\left[g^{-1} \alpha_{0} g, \eta\right]= & {\left[g^{-1}\left(d_{A_{0}} \alpha_{0}\right) g, \eta\right]+\left[\left[\left(A_{0}-g^{*} A_{0}\right) \wedge g^{-1} \alpha_{0} g\right], \eta\right] } \\
& -\left[g^{-1} \alpha_{0} g \wedge d_{A_{0}} \eta\right]
\end{aligned}
$$

and equation (7.14), we obtain

$$
\begin{aligned}
\left\|\left[g^{-1} \alpha_{0} g, \eta\right]\right\|_{1, p, \varepsilon}= & \left\|\left[g^{-1} \alpha_{0} g, \eta\right]\right\|_{L^{p}}+\varepsilon\left\|d_{A_{0}}\left[g^{-1} \alpha_{0} g, \eta\right]\right\|_{L^{p}} \\
& +\varepsilon\left\|d_{A_{0}}\left[* g^{-1} \alpha_{0} g, \eta\right]\right\|_{L^{p}} \\
\leq & c_{5} \varepsilon^{-1-2 / p}\left\|\zeta_{0}\right\|_{1, p, \varepsilon}\|\eta\|_{1, p, \varepsilon} .
\end{aligned}
$$

Similarly, using the identity

$$
d_{A_{0}}\left[\left(A_{0}-g^{*} A_{0}\right), \eta\right]=\left[d_{A_{0}}\left(A_{0}-g^{*} A_{0}\right), \eta\right]-\left[\left(A_{0}-g^{*} A_{0}\right) \wedge d_{A_{0}} \eta\right],
$$

and equation (7.14) we obtain

$$
\left\|\left[\left(A_{0}-g^{*} A_{0}\right), \eta\right]\right\|_{1, p, \varepsilon} \leq c_{6} \varepsilon^{-1-2 / p}\|\eta\|_{2, p, \varepsilon}\|\eta\|_{1, p, \varepsilon} .
$$

Hence, by equation (7.19),

$$
\left\|d_{g^{*} A} \eta-d_{A_{0}} \eta\right\|_{1, p, \varepsilon} \leq c_{7} \varepsilon^{-1-2 / p}\left(\left\|\zeta_{0}\right\|_{1, p, \varepsilon}+\|\eta\|_{2, p, \varepsilon}\right)\|\eta\|_{1, p, \varepsilon} .
$$

Moreover, since $Z\left(u_{0}\right)=L_{u_{0}}$, we have

$$
\begin{aligned}
& \left\|Z\left(g^{-1} u\right) \eta-L_{u_{0}} \eta\right\|_{1, p, \varepsilon} \\
& \quad \leq\left\|Z\left(g^{-1} u\right) \eta-Z(u) \eta\right\|_{1, p, \varepsilon}+\left\|Z(u) \eta-Z\left(u_{0}\right) \eta\right\|_{1, p, \varepsilon} \\
& \quad \leq c_{8}\left(\|\eta\|_{1, p, \varepsilon}\|\eta\|_{L^{\infty}}+\left\|\xi_{0}\right\|_{1, p, \varepsilon}\|\eta\|_{L^{\infty}}+\left\|\xi_{0}\right\|_{L^{\infty}}\|\eta\|_{1, p, \varepsilon}\right) \\
& \quad \leq c_{9} \varepsilon^{-2 / p}\left(\left\|\zeta_{0}\right\|_{1, p, \varepsilon}+\|\eta\|_{2, p, \varepsilon}\right)\|\eta\|_{1, p, \varepsilon} .
\end{aligned}
$$

Here we have used the inequality $\|\eta\|_{L^{\infty}} \leq c_{1} \delta$ from equation (7.5) and Lemma 4.4. The constants $c_{7}$ and $c_{9}$ in the last two estimates depend continuously on the pair $\left(u_{0}, A_{0}\right)$ with respect to the $C^{1}$-norm and are gauge-invariant. Hence, by Lemma 2.2, they can be chosen independent of $\left(u_{0}, A_{0}\right) \in \widetilde{\mathcal{M}}_{B, \Sigma}^{0}\left(c_{0}\right)$. Hence the second inequality in equation (7.16) follows from equation (7.18). To prove equation (7.17), we observe that

$$
\begin{aligned}
& d_{\left(u_{0}, A_{0}\right)}^{*_{\varepsilon}} \partial_{r} \zeta_{r}-d_{\left(u_{0}, A_{0}\right)}^{*_{\varepsilon}} d_{\left(u_{0}, A_{0}\right)} \eta \\
& \quad=\varepsilon^{2} d_{A_{0}}^{*}\left(d_{g^{*} A} \eta-d_{A_{0}} \eta\right)+L_{u_{0}}^{*}\left(Z\left(g^{-1} u\right) \eta-L_{u_{0}} \eta\right),
\end{aligned}
$$

where $(u, A)$ and $g:=e^{r \eta}$ are as in equation (7.15). The terms on the righthand side have been estimated above and this proves equation (7.17). Thus we have proved the existence of $\zeta_{1}$. The inequality (7.7) with $\delta$ sufficiently 
small guarantees that the $C^{0}$ distance between $u_{0}$ and $\exp _{u_{0}}\left(\xi_{1}\right)$ is smaller than the injectivity radius. This proves uniqueness.

Proof of Proposition 7.1. The proof is based on a Newton-type iteration. Let

$$
\left(u_{1}, A_{1}\right):=(u, A)=\left(\exp _{u_{0}}(\xi), A_{0}+\alpha\right), \quad \zeta_{1}:=\zeta .
$$

For $\nu \geq 2$, we define $\zeta_{\nu}=\left(\xi_{\nu}, \alpha_{\nu}\right) \in T_{\left(u_{0}, A_{0}\right)} \mathcal{B}$ inductively by

$$
\left(\exp _{u_{0}}\left(\xi_{\nu+1}\right), A_{0}+\alpha_{\nu+1}\right):=\left(u_{\nu+1}, A_{\nu+1}\right):=g_{\nu}^{*}\left(u_{\nu}, A_{\nu}\right)
$$

where $\left(u_{\nu}, A_{\nu}\right):=\left(\exp _{u_{0}}\left(\xi_{\nu}\right), A_{0}+\alpha_{\nu}\right), g_{\nu}:=e^{\hat{\eta}_{\nu}}$, and $\hat{\eta}_{\nu} \in \Omega^{0}\left(\Sigma, \mathfrak{g}_{P}\right)$ is the unique solution of the equation

$$
d_{\left(u_{0}, A_{0}\right)}^{* \varepsilon} d_{\left(u_{0}, A_{0}\right)} \hat{\eta}_{\nu}+d_{\left(u_{0}, A_{0}\right)}^{* \varepsilon} \zeta_{\nu}=0 .
$$

To construct these sequences, we must ensure that in each step $\zeta_{\nu}$ and $\hat{\eta}_{\nu}$ satisfy the hypotheses of Lemma 7.3 so that $\zeta_{\nu+1}$ can be chosen as in the assertion of Lemma 7.3. We shall prove this below. And we shall also prove that these sequences satisfy the following estimates:

$$
\begin{aligned}
\left\|\zeta_{\nu}\right\|_{1, p, \varepsilon} & \leq C\|\zeta\|_{1, p, \varepsilon}, \\
\left\|d_{\left(u_{0}, A_{0}\right)}^{*_{\varepsilon}} \zeta_{\nu}\right\|_{L^{p}} & \leq C \varepsilon^{-2 / p}\left\|\zeta_{\nu-1}\right\|_{1, p, \varepsilon}\left\|d_{\left(u_{0}, A_{0}\right)}^{*_{\varepsilon}} \zeta_{\nu-1}\right\|_{L^{p}}, \\
\left\|d_{\left(u_{0}, A_{0}\right)}^{*_{\varepsilon}} \zeta_{\nu}\right\|_{L^{p}} & \leq 2^{1-\nu}\left\|d_{\left(u_{0}, A_{0}\right)}^{*_{\varepsilon}} \zeta\right\|_{L^{p}} \\
\left\|\hat{\eta}_{\nu}\right\|_{2, p, \varepsilon} & \leq C 2^{-\nu}\|\zeta\|_{1, p, \varepsilon} .
\end{aligned}
$$

The constants $C$ and $\delta$ are chosen as follows. Suppose that the constants $c_{1}, c_{2}, c_{3}, c_{4}, c_{5} \geq 1$ and $\delta_{0}, \delta_{3}, \delta_{4} \in(0,1]$ satisfy the following conditions.

- The injectivity radius of $M$ on $u_{0}(P)$ is bigger than $\delta_{0}$.

- The inequality (7.9) holds with $c_{1}$ for every $x \in \mu^{-1}(0)$.

- The assertion of Lemma 7.2 holds for $0<\varepsilon \leq 1$ with $c$ replaced by $c_{2}$.

- The assertion of Lemma 7.3 holds for $0<\varepsilon \leq 1$ with $c$ replaced by $c_{3}$ and $\delta$ replaced by $\delta_{3}$.

- The assertion of Lemma 7.4 holds for $0<\varepsilon \leq 1$ with $c$ replaced by $c_{4}$ and $\delta$ replaced by $\delta_{4}$.

- The assertion of Lemma 4.4 holds for $0<\varepsilon \leq 1$ with $c$ replaced by $c_{5}$.

Now choose positive constants $C$ and $\delta$ such that

$$
\begin{aligned}
& c_{1} c_{2}\left(1+2 c_{2}\right) c_{3} \leq C, \quad 2 c_{2}\left(1+c_{2}\right) c_{3} C \delta \leq 1, \quad 2 C \delta \leq \delta_{3}, \\
& 4 c_{4} c_{5} C \delta \leq \delta_{4} \text {. }
\end{aligned}
$$

We prove that the estimates (7.20) to (7.23) hold for $\nu=1$. Since $C / 2 \geq$ $c_{1} c_{2}$, the inequality (7.23) with $\nu=1$ follows from Lemma 7.2. Since $C \geq 1$, the inequality (7.20) holds for $\nu=1$. The inequality (7.21) is vacuous for $\nu=1$ and (7.22) is obvious. 
Now suppose that the sequences have been constructed and the inequalities (7.20) to (7.23) have been established up to some integer $\nu \geq 1$. Then

$$
\left\|\hat{\eta}_{\nu}\right\|_{2, p, \varepsilon}+\left\|\zeta_{\nu}\right\|_{1, p, \varepsilon} \leq C\left(1+2^{-\nu}\right)\|\zeta\|_{1, p, \varepsilon} \leq 2 C \delta \varepsilon^{2 / p} \leq \delta_{3} \varepsilon^{2 / p} .
$$

Hence the hypotheses of Lemma 7.3 are satisfied with $\zeta_{0}$ replaced by $\zeta_{\nu}$ and $\eta$ replaced by $\hat{\eta}_{\nu}$. Choose $\zeta_{\nu+1}=\left(\xi_{\nu+1}, \alpha_{\nu+1}\right)$ as in the assertion of Lemma 7.3. By Lemma 7.2, we have

$$
\left\|\hat{\eta}_{\nu}\right\|_{1, p, \varepsilon} \leq c_{2}\left\|\zeta_{\nu}\right\|_{0, p, \varepsilon}, \quad\left\|\hat{\eta}_{\nu}\right\|_{2, p, \varepsilon} \leq c_{2}\left\|d_{\left(u_{0}, A_{0}\right)}^{*_{\varepsilon}} \zeta_{\nu}\right\|_{L^{p}} .
$$

Moreover, $d_{\left(u_{0}, A_{0}\right)}^{* \varepsilon}\left(\zeta_{\nu}+d_{\left(u_{0}, A_{0}\right)} \eta_{\nu}\right)=0$, and hence, by equation (7.8),

$$
\begin{aligned}
\left\|d_{\left(u_{0}, A_{0}\right)}^{*} \zeta_{\nu+1}\right\|_{L^{p}} & \leq c_{3} \varepsilon^{-2 / p}\left(\left\|\zeta_{\nu}\right\|_{1, p, \varepsilon}+\left\|\hat{\eta}_{\nu}\right\|_{2, p, \varepsilon}\right)\left\|\hat{\eta}_{\nu}\right\|_{1, p, \varepsilon} \\
& \leq c_{2}\left(1+c_{2}\right) c_{3} \varepsilon^{-2 / p}\left\|\zeta_{\nu}\right\|_{1, p, \varepsilon}\left\|d_{\left(u_{0}, A_{0}\right)}^{*_{\varepsilon}} \zeta_{\nu}\right\|_{L^{p}} .
\end{aligned}
$$

Since $c_{2}\left(1+c_{2}\right) c_{3} \leq C$, this proves equation (7.21) with $\nu$ replaced by $\nu+1$. Moreover, by equation (7.20),

$$
\begin{aligned}
\left\|d_{\left(u_{0}, A_{0}\right)}^{*_{\varepsilon}} \zeta_{\nu+1}\right\|_{L^{p}} & \leq c_{2}\left(1+c_{2}\right) c_{3} C \varepsilon^{-2 / p}\|\zeta\|_{1, p, \varepsilon}\left\|d_{\left(u_{0}, A_{0}\right)}^{*_{\varepsilon}} \zeta_{\nu}\right\|_{L^{p}} \\
& \leq c_{2}\left(1+c_{2}\right) c_{3} C \delta\left\|d_{\left(u_{0}, A_{0}\right)}^{*_{\varepsilon}} \zeta_{\nu}\right\|_{L^{p}} .
\end{aligned}
$$

Since $2 c_{2}\left(1+c_{2}\right) c_{3} C \delta \leq 1$, this proves equation (7.22) with $\nu$ replaced by $\nu+1$. Now let $\hat{\eta}_{\nu+1}$ be the unique solution of $d_{\left(u_{0}, A_{0}\right)}^{*} d_{\left(u_{0}, A_{0}\right)} \hat{\eta}_{\nu+1}+$ $d_{\left(u_{0}, A_{0}\right)}^{*} \zeta_{\nu+1}=0$. Then, by Lemma 7.2 and equation (7.22),

$$
\left\|\hat{\eta}_{\nu+1}\right\|_{2, p, \varepsilon} \leq c_{2}\left\|d_{\left(u_{0}, A_{0}\right)}^{*_{\varepsilon}} \zeta_{\nu+1}\right\|_{L^{p}} \leq c_{2} 2^{-\nu}\left\|d_{\left(u_{0}, A_{0}\right)}^{*_{\varepsilon}} \zeta\right\|_{L^{p}} \leq c_{1} c_{2} 2^{-\nu}\|\zeta\|_{1, p, \varepsilon} .
$$

Since $2 c_{1} c_{2} \leq C$ this implies equation (7.23) with $\nu$ replaced by $\nu+1$. It remains to prove equation (7.20) with $\nu$ replaced by $\nu+1$. By equations (7.7) and (7.22), we have

$$
\left\|\zeta_{j+1}-\zeta_{j}\right\|_{1, p, \varepsilon} \leq c_{3}\left\|\hat{\eta}_{j}\right\|_{2, p, \varepsilon} \leq c_{2} c_{3} 2^{1-j}\left\|d_{\left(u_{0}, A_{0}\right)}^{*_{\varepsilon}} \zeta\right\|_{L^{p}} \leq c_{1} c_{2} c_{3} 2^{1-j}\|\zeta\|_{1, p, \varepsilon}
$$

for $j=1, \ldots, \nu$. Hence

$$
\left\|\zeta_{\nu+1}\right\|_{1, p, \varepsilon} \leq\|\zeta\|_{1, p, \varepsilon}+\sum_{j=1}^{\nu}\left\|\zeta_{j+1}-\zeta_{j}\right\|_{1, p, \varepsilon} \leq\left(1+2 c_{1} c_{2} c_{3}\right)\|\zeta\|_{1, p, \varepsilon}
$$

Since $1+2 c_{1} c_{2} c_{3} \leq C$ this proves equation (7.20) with $\nu$ replaced by $\nu+1$. This completes the induction.

By equation (7.24), $\zeta_{\nu}$ is a Cauchy sequence in the $W^{1, p}$-norm. Moreover,

$$
\left(u_{\nu}, A_{\nu}\right)=\left(\exp _{u_{0}}\left(\xi_{\nu}\right), A_{0}+\alpha_{\nu}\right)=h_{\nu}^{*}(u, A),
$$


where $h_{\nu}:=g_{1} g_{2} \cdots g_{\nu-1}$. We prove by induction that there exists a sequence $\eta_{\nu} \in \Omega^{0}\left(\Sigma, \mathfrak{g}_{P}\right)$ such that

$$
h_{\nu}=e^{\eta_{\nu}}, \quad\left\|\eta_{\nu+1}-\eta_{\nu}\right\|_{2, p, \varepsilon} \leq c_{4} C 2^{-\nu}\|\zeta\|_{1, p, \varepsilon} .
$$

For $\nu=1$ we set $h_{1}:=\mathbb{1}$ and $\eta_{1}:=0$. Suppose that the sequence has been constructed for all integers up to $\nu \geq 1$. Then

$$
\left\|\eta_{\nu}\right\|_{2, p, \varepsilon} \leq \sum_{j=1}^{\nu-1}\left\|\eta_{j+1}-\eta_{j}\right\|_{2, p, \varepsilon} \leq c_{4} C\|\zeta\|_{1, p, \varepsilon} .
$$

Hence, by Lemma 4.4 and equations (7.1) and (7.23)

$$
\begin{gathered}
\left\|\eta_{\nu}\right\|_{L^{\infty}} \leq c_{5} \varepsilon^{-2 / p}\left\|\eta_{\nu}\right\|_{2, p, \varepsilon} \leq c_{4} c_{5} C \varepsilon^{-2 / p}\|\zeta\|_{1, p, \varepsilon} \leq c_{4} c_{5} C \delta \leq \frac{\delta_{4}}{4} \\
\left\|\hat{\eta}_{\nu}\right\|_{L^{\infty}} \leq c_{5} \varepsilon^{-2 / p}\left\|\hat{\eta}_{\nu}\right\|_{2, p, \varepsilon} \leq c_{5} C \varepsilon^{-2 / p}\|\zeta\|_{1, p, \varepsilon} \leq c_{5} C \delta \leq \frac{\delta_{4}}{4}
\end{gathered}
$$

By Lemma 7.4 , there exists a section $\eta_{\nu+1} \in \Omega^{0}\left(\Sigma, \mathfrak{g}_{P}\right)$ such that

$$
e^{\eta_{\nu+1}}=e^{\eta_{\nu}} e^{\hat{\eta}_{\nu}}=h_{\nu} g_{\nu}=h_{\nu+1}, \quad\left\|\eta_{\nu+1}\right\|_{L^{\infty}} \leq 2\left\|\eta_{\nu}+\hat{\eta}_{\nu}\right\|_{L^{\infty}} \leq \delta_{4} .
$$

Applying Lemma 7.4 to $-\eta_{\nu}$ and $\eta_{\nu+1}$, we find

$$
\left\|\eta_{\nu+1}-\eta_{\nu}\right\|_{2, p, \varepsilon} \leq c_{4}\left\|\hat{\eta}_{\nu}\right\|_{2, p, \varepsilon} \leq c_{4} C 2^{-\nu}\|\zeta\|_{1, p, \varepsilon} .
$$

The last inequality follows from equation (7.23). This completes the induction. Thus we have proved that $h_{\nu}$ satisfies equations (7.25) and hence is a Cauchy sequence in $\mathcal{G}^{2, p}(P)$. Denote

$$
\zeta:=\lim _{\nu \rightarrow \infty} \zeta_{\nu}, \quad h:=\lim _{\nu \rightarrow \infty} h_{\nu}, \quad \eta:=\lim _{\nu \rightarrow \infty} \eta_{\nu}
$$

Then

$$
e^{\eta}=h, \quad h^{*}(u, A)=\left(\exp _{u_{0}}(\xi), A_{0}+\alpha\right), \quad d_{\left(u_{0}, A_{0}\right)}^{*} \zeta=0 .
$$

The last equation follows from equation (7.22). Moreover, by equations (7.20) and (7.26), we have $\|\eta\|_{2, p, \varepsilon}+\|\zeta\|_{1, p, \varepsilon} \leq C\left(1+c_{4}\right)\|\zeta\|_{1, p, \varepsilon}$. Hence equation (7.2) holds with $c:=C\left(1+c_{4}\right)$.

To complete the existence proof we must show that $\eta$ and $\zeta$ are smooth. We shall prove that the sequence $\zeta_{\nu}$ is bounded on $W^{k, p}$ for every $k$. Here it suffices to obtain rather crude estimates with constants which depend on $\varepsilon$ and are allowed to diverge as $\varepsilon$ tends to zero. We fix a constant $\varepsilon>0$ and prove by induction that for every integer $k \geq 1$ there exists a constant $c_{k}=c_{k}\left(p, \varepsilon, u_{0}, A_{0}, u, A\right)$ such that, for every $\nu$,

$$
\left\|\zeta_{\nu}\right\|_{W^{k, p}} \leq c_{k}, \quad\left\|\hat{\eta}_{\nu}\right\|_{W^{k+1, p}} \leq c_{k} 2^{-\nu}
$$


For $k=1$ this follows from equations (7.20) and (7.23). Now let $k \geq 2$ and assume that these estimates have been established with $k$ replaced by $k-1$. Observe that there exists a constant $C_{k} \geq 1$ such that, for every $\nu$,

$$
\begin{aligned}
\left\|\zeta_{\nu+1}-\zeta_{\nu}\right\|_{W^{k, p}} & \leq C_{k}\left\|\hat{\eta}_{\nu}\right\|_{W^{k+1, p}} \\
\left\|\hat{\eta}_{\nu}\right\|_{W^{k+1, p}} & \leq C_{k}\left\|d_{\left(u_{0}, A_{0}\right)}^{*_{\varepsilon}} \zeta_{\nu}\right\|_{W^{k-1, p}}, \\
\left\|d_{\left(u_{0}, A_{0}\right)}^{*_{\varepsilon}} \zeta_{\nu+1}\right\|_{W^{k-1, p}} & \leq C_{k}\left(\left\|\zeta_{\nu}\right\|_{W^{k, p}}+\left\|\hat{\eta}_{\nu}\right\|_{W^{k+1, p}}\right)\left\|\hat{\eta}_{\nu}\right\|_{W^{k, p}} .
\end{aligned}
$$

The first two inequalities are obvious and the last follows by inspecting the formula (7.18) in the proof of Lemma 7.3. Combining these inequalities with the induction hypothesis, we obtain

$$
\begin{aligned}
\left\|\zeta_{\nu+1}\right\|_{W^{k, p}} & \leq\left\|\zeta_{\nu}\right\|_{W^{k, p}}+C_{k}\left\|\hat{\eta}_{\nu}\right\|_{W^{k+1, p}} \\
\left\|\hat{\eta}_{\nu+1}\right\|_{W^{k+1, p}} & \leq C_{k}^{2} c_{k-1}\left(\left\|\zeta_{\nu}\right\|_{W^{k, p}}+\left\|\hat{\eta}_{\nu}\right\|_{W^{k+1, p}}\right) 2^{-\nu}
\end{aligned}
$$

Abbreviate

$$
a_{\nu}:=\left\|\zeta_{\nu+\nu_{0}}\right\|_{W^{k, p}}+C_{k}\left\|\hat{\eta}_{\nu+\nu_{0}}\right\|_{W^{k+1, p}}
$$

and choose $\nu_{0}$ so large that $C_{k}^{3} c_{k-1} 2^{-\nu_{0}} \leq 1$. Then

$$
\begin{aligned}
a_{\nu+1} & \leq\left\|\zeta_{\nu+\nu_{0}}\right\|_{W^{k, p}}+C_{k}\left\|\hat{\eta}_{\nu+\nu_{0}}\right\|_{W^{k+1, p}}+C_{k}\left\|\hat{\eta}_{\nu+\nu_{0}+1}\right\|_{W^{k+1, p}} \\
& =a_{\nu}+C_{k}\left\|\hat{\eta}_{\nu+\nu_{0}+1}\right\|_{W^{k+1, p}} \\
& \leq a_{\nu}+C_{k}^{3} c_{k-1}\left(\left\|\zeta_{\nu}\right\|_{W^{k, p}}+\left\|\hat{\eta}_{\nu}\right\|_{W^{k+1, p}}\right) 2^{-\nu-\nu_{0}} \\
& \leq\left(1+2^{-\nu}\right) a_{\nu}
\end{aligned}
$$

for all $\nu$ and hence the sequence $a_{\nu}$ is bounded. It follows that the sequences $\left\|\zeta_{\nu}\right\|_{W^{k, p}}$ and $2^{\nu}\left\|\hat{\eta}_{\nu}\right\|_{W^{k+1, p}}$ are bounded. Thus we have proved that $\hat{\eta}_{\nu}$ and $\zeta_{\nu}$ satisfy equation (7.27). This completes the induction. It follows that $\zeta$ is smooth and hence, so is $\eta$. This proves existence.

We prove uniqueness. Choose $\delta>0$ so small that

$$
c_{5} c \delta \leq \delta_{0}, \quad 2 c_{4} c \delta \leq \delta_{3}, \quad c_{5} c \delta \leq \delta_{4}, \quad 2 c_{2} c_{3} c_{4} c \delta<1 .
$$

Assume that $\zeta_{0}, \zeta_{1} \in T_{\left(u_{0}, A_{0}\right)} \mathcal{B}$ and $\eta_{0}, \eta_{1} \in \Omega^{0}\left(\Sigma, \mathfrak{g}_{P}\right)$ satisfy the requirements of the proposition. Then

$$
d_{\left(u_{0}, A_{0}\right)}^{*_{\varepsilon}} \zeta_{i}=0, \quad g_{i}^{*}(u, A)=\left(\exp _{u_{0}}\left(\xi_{i}\right), A_{0}+\alpha_{i}\right)
$$

for $i=0,1$, where $g_{i}:=e^{\eta_{i}}$. By Lemma 4.4, we have

$$
\left\|\eta_{i}\right\|_{L^{\infty}} \leq c_{5} \varepsilon^{-2 / p}\left\|\eta_{i}\right\|_{2, p, \varepsilon} \leq c_{5} c \varepsilon^{-2 / p}\|\zeta\|_{1, p, \varepsilon} \leq c_{5} c \delta \leq \delta_{4}
$$

for $i=0,1$. Hence, by Lemma 7.4, there exists a unique element $\eta \in$ $\Omega^{0}\left(\Sigma, \mathfrak{g}_{P}\right)$ such that

$$
g:=e^{\eta}=g_{0}^{-1} g_{1}, \quad c_{4}^{-1}\left\|\eta_{1}-\eta_{0}\right\|_{2, p, \varepsilon} \leq\|\eta\|_{2, p, \varepsilon} \leq c_{4}\left\|\eta_{1}-\eta_{0}\right\|_{2, p, \varepsilon} .
$$

The gauge transformation $g$ satisfies

$$
g^{*}\left(\exp _{u_{0}}\left(\xi_{0}\right), A_{0}+\alpha_{0}\right)=\left(\exp _{u_{0}}\left(\xi_{1}\right), A_{0}+\alpha_{1}\right)
$$


Moreover,

$$
\left\|\zeta_{0}\right\|_{1, p, \varepsilon}+\|\eta\|_{2, p, \varepsilon} \leq 2 c_{4} c\|\zeta\|_{1, p, \varepsilon} \leq 2 c_{4} c \delta \varepsilon^{2 / p} \leq \delta_{3} \varepsilon^{2 / p} .
$$

Hence $\zeta_{0}$ and $\eta$ satisfy the hypotheses of Lemma 7.3. We use Lemma 7.2 and the estimate (7.8) of Lemma 7.3 to obtain

$$
\begin{aligned}
\|\eta\|_{2, p, \varepsilon} & \leq c_{2}\left\|d_{\left(u_{0}, A_{0}\right)}^{*_{\varepsilon}} d_{\left(u_{0}, A_{0}\right)} \eta\right\|_{L^{p}} \\
& \leq c_{2} c_{3} \varepsilon^{-2 / p}\left(\left\|\zeta_{1}-\zeta_{0}\right\|_{1, p, \varepsilon}+\|\eta\|_{2, p, \varepsilon}\right)\|\eta\|_{1, p, \varepsilon} \\
& \leq c_{2} c_{3} c_{4} \varepsilon^{-2 / p}\left(\left\|\zeta_{1}-\zeta_{0}\right\|_{1, p, \varepsilon}+\left\|\eta_{1}-\eta_{0}\right\|_{2, p, \varepsilon}\right)\|\eta\|_{1, p, \varepsilon} \\
& \leq 2 c_{2} c_{3} c_{4} c \varepsilon^{-2 / p}\|\zeta\|_{1, p, \varepsilon}\|\eta\|_{1, p, \varepsilon} \\
& \leq 2 c_{2} c_{3} c_{4} c \delta\|\eta\|_{1, p, \varepsilon} .
\end{aligned}
$$

Since $2 c_{2} c_{3} c_{4} c \delta<1$, we have $\eta=0$ and hence $\eta_{1}=\eta_{0}$. Hence $\alpha_{0}=\alpha_{1}$ and $\exp _{u_{0}}\left(\xi_{0}\right)=\exp _{u_{0}}\left(\xi_{1}\right)$. By equations (7.1) and (7.2), and Lemma 4.4, we have

$$
\left\|\xi_{i}\right\|_{L^{\infty}} \leq c_{5} \varepsilon^{-2 / p}\left\|\zeta_{i}\right\|_{1, p, \varepsilon} \leq c_{5} c \varepsilon^{-2 / p}\|\zeta\|_{1, p, \varepsilon} \leq c_{5} c \delta \leq \delta_{0}
$$

for $i=0,1$. Hence $\xi_{0}=\xi_{1}$.

\section{Proof of Theorem 3.2}

In this section, we prove that the map $\widetilde{\mathcal{T}}^{\varepsilon}: \widetilde{\mathcal{M}}_{B, \Sigma}^{0}\left(c_{0}\right) \rightarrow \widetilde{\mathcal{M}}_{B, \Sigma}^{\varepsilon}$ introduced in Theorem 6.1 is locally surjective. This is the content of Theorem 3.2 and is restated more precisely as follows.

Theorem 8.1. Assume $(H 1)$ and $(H 4)$ and let $\bar{B} \in H_{2}(\bar{M} ; \mathbb{Z})$ be a nontorsion homology class. Then, for every $c_{0}>0$ and every $p>2$, there exist positive constants $\varepsilon_{0}$ and $\delta$ such that the following holds for every $\varepsilon \in\left(0, \varepsilon_{0}\right]$. If $\left(\bar{u}_{0}, \bar{A}_{0}\right) \in \widetilde{\mathcal{M}}_{B, \Sigma}^{0}\left(c_{0}-1\right)$ and $(u, A)=\left(\exp _{\bar{u}_{0}}(\bar{\xi}), \bar{A}_{0}+\bar{\alpha}\right) \in \widetilde{\mathcal{M}}_{B, \Sigma}^{\varepsilon}$ where $\bar{\zeta}=(\bar{\xi}, \bar{\alpha}) \in T_{\left(\bar{u}_{0}, \bar{A}_{0}\right)} \mathcal{B}$ satisfies

$$
\|\bar{\zeta}\|_{1, p, \varepsilon ;\left(\bar{u}_{0}, \bar{A}_{0}\right)} \leq \delta \varepsilon^{2 / p+1 / 2}
$$

then there exist $\bar{\xi}_{0} \in \operatorname{ker} \mathcal{D}_{\left(\bar{u}_{0}, \bar{A}_{0}\right)}^{0}$ and $\eta_{0} \in \Omega^{0}\left(\Sigma, \mathfrak{g}_{P}\right)$ such that

$$
\begin{gathered}
g^{*}(u, A)=\tilde{\mathcal{T}}^{\varepsilon}\left(u_{0}, A_{0}\right), \quad g:=e^{\eta_{0}}, \quad\left(u_{0}, A_{0}\right):=\mathcal{F}_{\left(\bar{u}_{0}, \bar{A}_{0}\right)}^{0}\left(\bar{\xi}_{0}\right), \\
\left\|\bar{\xi}_{0}\right\|_{W^{1, p}}+\left\|\eta_{0}\right\|_{2, p, \varepsilon ; A_{0}} \leq c\|\bar{\zeta}\|_{1, p, \varepsilon ;\left(\bar{u}_{0}, \bar{A}_{0}\right)} .
\end{gathered}
$$

Here $\mathcal{F}_{\left(\bar{u}_{0}, \bar{A}_{0}\right)}^{0}$ is the map of Theorem 2.1. The proof of Theorem 8.1 is based on Corollary 6.3 and on the construction of a tubular neighbourhood of the moduli space $\mathcal{M}_{B, \Sigma}^{0}\left(c_{0}\right)$ in the quotient $\mathcal{B} / \mathcal{G}$ (Figure 1$)$. 


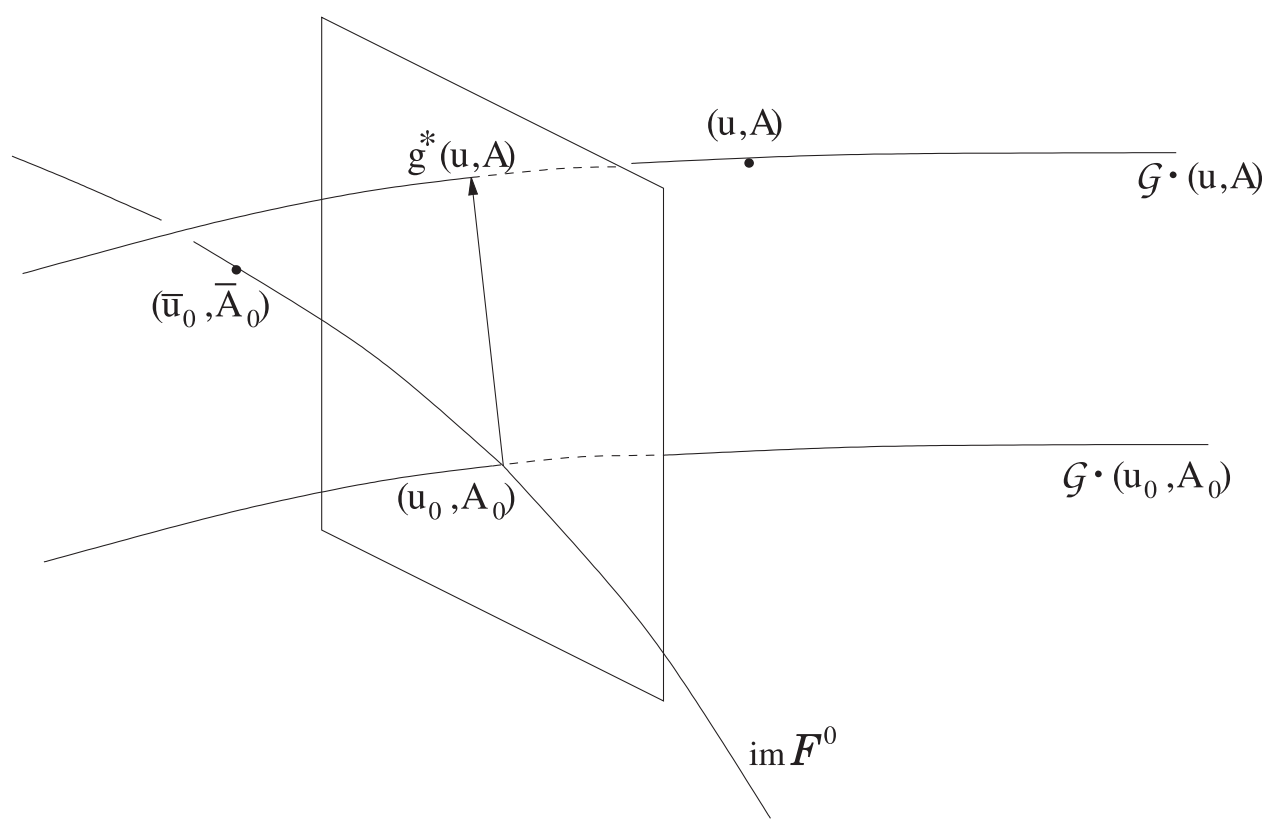

Figure 1. A tubular neighbourhood of $\mathcal{M}^{0}$.

Proposition 8.2. Assume $(H 1)$ and $(H 4)$ and let $\bar{B} \in H_{2}(\bar{M} ; \mathbb{Z})$ be a nontorsion homology class. For every $p>2$ and every $c_{0}>0$, there exist positive constants $\delta, \varepsilon_{0}$, and $c$ such that, for every $\varepsilon \in\left(0, \varepsilon_{0}\right]$, the following holds. Let $\left(\bar{u}_{0}, \bar{A}_{0}\right) \in \widetilde{\mathcal{M}}_{B, \Sigma}^{0}\left(c_{0}-1\right)$ and $(u, A)=\left(\exp _{\bar{u}_{0}}(\bar{\xi}), \bar{A}_{0}+\bar{\alpha}\right)$, where the pair $\bar{\zeta}:=(\bar{\xi}, \bar{\alpha}) \in T_{\left(\bar{u}_{0}, \bar{A}_{0}\right)} \mathcal{B}$ satisfies

$$
\|\bar{\zeta}\|_{1, p, \varepsilon ;\left(\bar{u}_{0}, \bar{A}_{0}\right)} \leq \delta \varepsilon^{2 / p} .
$$

Then there exist $\bar{\xi}_{0} \in \operatorname{ker} \mathcal{D}_{\left(\bar{u}_{0}, \bar{A}_{0}\right)}^{0}, \eta_{0} \in \Omega^{0}\left(\Sigma, \mathfrak{g}_{P}\right),\left(u_{0}, A_{0}\right) \in \widetilde{\mathcal{M}}_{B, \Sigma}^{0}\left(c_{0}\right)$, and $\zeta_{0}=\left(\xi_{0}, \alpha_{0}\right) \in T_{\left(u_{0}, A_{0}\right)} \mathcal{B}$, such that

$$
g^{*}(u, A)=\left(\exp _{u_{0}}\left(\xi_{0}\right), A_{0}+\alpha_{0}\right), \quad\left(u_{0}, A_{0}\right)=\mathcal{F}_{\left(\bar{u}_{0}, \bar{A}_{0}\right)}^{0}\left(\bar{\xi}_{0}\right),
$$

where $g:=e^{\eta_{0}}$, and

$$
\begin{gathered}
d_{\left(u_{0}, A_{0}\right)}^{*_{\varepsilon}} \zeta_{0}=0, \quad \zeta_{0} \in \operatorname{im}\left(\mathcal{D}_{\left(u_{0}, A_{0}\right)}^{\varepsilon}\right)^{*} \\
\left\|\bar{\xi}_{0}\right\|_{W^{1, p}}+\left\|\eta_{0}\right\|_{2, p, \varepsilon ; \bar{A}_{0}}+\left\|\zeta_{0}\right\|_{1, p, \varepsilon ;\left(u_{0}, A_{0}\right)} \leq c\|\bar{\zeta}\|_{1, p, \varepsilon ;\left(\bar{u}_{0}, \bar{A}_{0}\right)} .
\end{gathered}
$$

The proof of Proposition 8.2 is based on Proposition 7.1. The latter can be restated as follows. Let $\mathcal{H}^{\varepsilon} \subset T \mathcal{B}$ denote the horizontal subbundle with fibres

$$
\mathcal{H}_{(u, A)}^{\varepsilon}:=\operatorname{ker} d_{(u, A)}^{* \varepsilon} \subset T_{(u, A)} \mathcal{B}
$$


Given a pair $(u, A) \in \mathcal{B}$ and constants $p, c_{0}, \delta, \varepsilon$ denoted by $\mathcal{U}^{0}=\mathcal{U}^{0}(\delta, \varepsilon) \subset$ $\widetilde{\mathcal{M}}_{B, \Sigma}^{0}\left(c_{0}\right)$ the open set

$$
\mathcal{U}^{0}:=\left\{\left(u_{0}, A_{0}\right) \in \widetilde{\mathcal{M}}_{B, \Sigma}^{0}\left(c_{0}\right) \mid(u, A)=\left(\exp _{u_{0}}(\xi), A_{0}+\alpha\right),\|\zeta\|_{1, p, \varepsilon} \leq \delta \varepsilon^{2 / p}\right\} .
$$

If $\delta$ and $\varepsilon$ are sufficiently small, then Proposition 7.1 defines two maps

$$
\mathcal{S}^{\varepsilon}: \mathcal{U}^{0} \rightarrow \mathcal{H}^{\varepsilon}, \quad \mathcal{N}^{\varepsilon}: \mathcal{U}^{0} \rightarrow \Omega^{0}\left(\Sigma, \mathfrak{g}_{P}\right)
$$

such that $\mathcal{S}^{\varepsilon}$ is a section of $\mathcal{H}^{\varepsilon}$ over $\mathcal{U}^{0}$ and, for every $\left(u_{0}, A_{0}\right) \in \mathcal{U}^{0}$, the pair $\left(\xi_{0}, \alpha_{0}\right):=\mathcal{S}^{\varepsilon}\left(u_{0}, A_{0}\right)$ and the gauge transformation $g:=e^{\eta}$, where $\eta:=\mathcal{N}^{\varepsilon}\left(u_{0}, A_{0}\right)$, satisfy $g^{*}(u, A)=\left(\exp _{u_{0}}\left(\xi_{0}\right), A_{0}+\alpha_{0}\right)$ and equation (7.2). In particular,

$$
\left\|\mathcal{S}^{\varepsilon}\left(u_{0}, A_{0}\right)\right\|_{1, p, \varepsilon} \leq c\|\zeta\|_{1, p, \varepsilon},
$$

where $(u, A)=\left(\exp _{u_{0}}(\xi), A_{0}+\alpha\right)$ and $\|\zeta\|_{1, p, \varepsilon} \leq \delta \varepsilon^{2 / p}$. In this notation, Proposition 8.2 asserts that for every $(u, A) \in \mathcal{B}$, whose distance to $\widetilde{\mathcal{M}}_{B, \Sigma}^{0}$ in the $(1, p, \varepsilon)$-norm is less than $\delta \varepsilon^{2 / p}$ for a sufficiently small constant $\delta$, there exists a pair $\left(u_{0}, A_{0}\right) \in \mathcal{U}^{0}$ such that $\mathcal{S}^{\varepsilon}\left(u_{0}, A_{0}\right)$ lies in the image of $\left(\mathcal{D}_{\left(u_{0}, A_{0}\right)}^{\varepsilon}\right)^{*}$.

Lemma 8.3. For every $p>2$ and every $c_{0}>0$, there exist positive constants $\delta, \varepsilon_{0}$, and $c$ such that the following holds for every $\varepsilon \in\left(0, \varepsilon_{0}\right]$. Let $(u, A) \in \mathcal{B}$ and $I \subset \mathbb{R}$ be an interval. Suppose that $I \rightarrow \mathcal{U}^{0}(\delta, \varepsilon): r \mapsto\left(u_{0}(r), A_{0}(r)\right)$ is a smooth path, and let $\zeta(r)=(\xi(r), \alpha(r)) \in T_{\left(u_{0}(r), A_{0}(r)\right)} \mathcal{B}$ be the corresponding vector field along this path that satisfies

$$
(u, A)=\left(\exp _{u_{0}}(\xi), A_{0}+\alpha\right), \quad\|\zeta\|_{1, p, \varepsilon} \leq \delta \varepsilon^{2 / p} .
$$

Denote $\zeta_{\varepsilon}(r):=\mathcal{S}^{\varepsilon}\left(u_{0}(r), A_{0}(r)\right)$ and $\tilde{\nabla}_{r} \zeta_{\varepsilon}:=\left(\nabla_{r} \xi_{\varepsilon}-1 / 2 J\left(\nabla_{\partial_{r} u_{0}} J\right) \xi_{\varepsilon}, \partial_{r} \alpha_{\varepsilon}\right)$. Then

$$
\left\|\left(\partial_{r} u_{0}, \partial_{r} A_{0}\right)+\tilde{\nabla}_{r} \zeta_{\varepsilon}\right\|_{1, p, \varepsilon} \leq c\left(\|\zeta\|_{1, p, \varepsilon}+\left\|\varepsilon^{2} d_{A_{0}}^{*} \partial_{r} A_{0}-L_{u_{0}}^{*} \partial_{r} u_{0}\right\|_{L^{p}}\right) .
$$

Proof. Let $g(r):=e^{\eta_{\varepsilon}(r)}$, where $\eta_{\varepsilon}(r):=\mathcal{N}^{\varepsilon}\left(u_{0}(r), A_{0}(r)\right) \in \Omega^{0}\left(\Sigma, \mathfrak{g}_{P}\right)$, and denote $\dot{u}_{0}:=\partial_{r} u_{0}, \dot{A}_{0}:=\partial_{r} A_{0}, \dot{g}:=\partial_{r} g$. Let $\delta_{1}$ and $c_{1}$ be the constants of Proposition 7.1. Then

$$
\begin{gathered}
\left\|\eta_{\varepsilon}(r)\right\|_{2, p, \varepsilon}+\left\|\zeta_{\varepsilon}(r)\right\|_{1, p, \varepsilon} \leq c_{1}\|\zeta(r)\|_{1, p, \varepsilon} \leq c_{1} \delta_{1} \varepsilon^{2 / p} \\
g^{-1} u=\exp _{u_{0}}\left(\xi_{\varepsilon}\right), \quad g^{*} A=A_{0}+\alpha_{\varepsilon}, \quad \varepsilon^{2} d_{A_{0}}^{*} \alpha_{\varepsilon}-L_{u_{0}}^{*} \xi_{\varepsilon}=0 .
\end{gathered}
$$

Differentiating these identities, we obtain

$$
-L_{g^{-1} u}\left(g^{-1} \dot{g}\right)=E_{1} \dot{u}_{0}+E_{2} \nabla_{r} \xi_{\varepsilon}, \quad d_{g^{*} A}\left(g^{-1} \dot{g}\right)=\dot{A}_{0}+\dot{\alpha}_{\varepsilon},
$$

where $E_{1}:=E_{1}\left(u_{0}, \xi_{\varepsilon}\right)$ and $E_{2}:=E_{2}\left(u_{0}, \xi_{\varepsilon}\right)$ (see Appendix C), and

$$
\varepsilon^{2} d_{A_{0}}^{*} \dot{\alpha}_{\varepsilon}-\varepsilon^{2} *\left[\dot{A}_{0} \wedge * \alpha_{\varepsilon}\right]-L_{u_{0}}^{*} \nabla_{r} \xi_{\varepsilon}-\rho\left(\dot{u}_{0}, \xi_{\varepsilon}\right)=0,
$$


where $\rho \in \Omega^{2}(M, \mathfrak{g})$ is given by $\left\langle\eta, \rho\left(\xi_{1}, \xi_{2}\right)\right\rangle:=\left\langle\nabla_{\xi_{1}} X_{\eta}, \xi_{2}\right\rangle$ (see Lemma C.2). Inserting the expressions for $\nabla_{r} \xi_{\varepsilon}$ and $\dot{\alpha}_{\varepsilon}$ from equations (8.7) into equation (8.8) gives

$$
\begin{aligned}
& \varepsilon^{2} d_{A_{0}}^{*} d_{g^{*} A}\left(g^{-1} \dot{g}\right)+L_{u_{0}}^{*} E_{2}^{-1} L_{g^{-1} u}\left(g^{-1} \dot{g}\right) \\
& \quad=\varepsilon^{2} d_{A_{0}}^{*} \dot{A}_{0}+\varepsilon^{2} *\left[\dot{A}_{0} \wedge * \alpha_{\varepsilon}\right]-L_{u_{0}}^{*} E_{2}^{-1} E_{1} \dot{u}_{0}+\rho\left(\dot{u}_{0}, \xi_{\varepsilon}\right) .
\end{aligned}
$$

Since $g^{-1} u=\exp _{u_{0}}\left(\xi_{\varepsilon}\right)$ and $g^{*} A=A_{0}+\alpha_{\varepsilon}$ we have, by Lemma C.1,

$$
\begin{aligned}
E_{2}^{-1} L_{g^{-1} u}\left(g^{-1} \dot{g}\right) & =L_{u_{0}}\left(g^{-1} \dot{g}\right)+\left(E_{2}^{-1} E_{1}-\mathbb{1}\right) L_{u_{0}}\left(g^{-1} \dot{g}\right)+\nabla_{\xi_{\varepsilon}} X_{g^{-1}}\left(u_{0}\right), \\
d_{A_{0}}^{*} d_{g^{*} A}\left(g^{-1} \dot{g}\right) & =d_{A_{0}}^{*} d_{A_{0}}\left(g^{-1} \dot{g}\right)+\left[d_{A_{0}}^{*} \alpha_{\varepsilon}, g^{-1} \dot{g}\right]+*\left[* \alpha_{\varepsilon} \wedge d_{A_{0}}\left(g^{-1} \dot{g}\right)\right] .
\end{aligned}
$$

Hence

$$
\begin{aligned}
\varepsilon^{2} d_{A_{0}}^{*} & d_{A_{0}}\left(g^{-1} \dot{g}\right)+L_{u_{0}}^{*} L_{u_{0}}\left(g^{-1} \dot{g}\right) \\
= & -\varepsilon^{2}\left[d_{A_{0}}^{*} \alpha_{\varepsilon}, g^{-1} \dot{g}\right]-\varepsilon^{2} *\left[* \alpha_{\varepsilon} \wedge d_{A_{0}}\left(g^{-1} \dot{g}\right)\right] \\
& -L_{u_{0}}^{*}\left(E_{2}^{-1} E_{1}-\mathbb{1}\right) L_{u_{0}}\left(g^{-1} \dot{g}\right)-L_{u_{0}}^{*} \nabla_{\xi_{\varepsilon}} X_{g^{-1}}\left(u_{0}\right) \\
& +\varepsilon^{2} *\left[\dot{A}_{0} \wedge * \alpha_{\varepsilon}\right]+L_{u_{0}}^{*}\left(\mathbb{1}-E_{2}^{-1} E_{1}\right) \dot{u}_{0}+\rho\left(\dot{u}_{0}, \xi_{\varepsilon}\right) \\
& +\varepsilon^{2} d_{A_{0}}^{*} \dot{A}_{0}-L_{u_{0}}^{*} \dot{u}_{0} .
\end{aligned}
$$

By Lemma 7.2 and Lemma 4.4, there exists a constant $c_{2}>0$ such that

$$
\begin{aligned}
\left\|g^{-1} \dot{g}\right\|_{2, p, \varepsilon} \leq & c_{2} \varepsilon^{-2 / p}\left\|\zeta_{\varepsilon}\right\|_{1, p, \varepsilon}\left\|g^{-1} \dot{g}\right\|_{2, p, \varepsilon} \\
& +c_{2}\left(\left\|\zeta_{\varepsilon}\right\|_{0, p, \varepsilon}+\left\|\varepsilon^{2} d_{A_{0}}^{*} \dot{A}_{0}-L_{u_{0}}^{*} \dot{u}_{0}\right\|_{L^{p}}\right) \\
\leq & c_{1} c_{2} \delta_{1}\left\|g^{-1} \dot{g}\right\|_{2, p, \varepsilon} \\
& +c_{2}\left(\left\|\zeta_{\varepsilon}\right\|_{0, p, \varepsilon}+\left\|\varepsilon^{2} d_{A_{0}}^{*} \dot{A}_{0}-L_{u_{0}}^{*} \dot{u}_{0}\right\|_{L^{p}}\right) .
\end{aligned}
$$

The last inequality follows from equation (8.6). With $c_{1} c_{2} \delta_{1} \leq 1 / 2$, it follows that

$$
\left\|g^{-1} \dot{g}\right\|_{2, p, \varepsilon} \leq 2 c_{2}\left(\left\|\zeta_{\varepsilon}\right\|_{0, p, \varepsilon}+\left\|\varepsilon^{2} d_{A_{0}}^{*} \dot{A}_{0}-L_{u_{0}}^{*} \dot{u}_{0}\right\|_{L^{p}}\right) \text {. }
$$

Hence equation (8.5) follows from equation (7.5) and (8.7).

Consider the vector bundle

$$
\mathcal{V}^{0} \longrightarrow \widetilde{\mathcal{M}}_{B, \Sigma}^{0}
$$

whose fibre over $\left(u_{0}, A_{0}\right) \in \widetilde{\mathcal{M}}_{B, \Sigma}^{0}$ is the finite-dimensional vector space $\mathcal{V}_{\left(u_{0}, A_{0}\right)}^{0}$ of all pairs $\left(\xi_{0}, \alpha_{0}\right) \in \Omega^{0}\left(\Sigma, H_{u_{0}}\right) \times \Omega^{1}\left(\Sigma, \mathfrak{g}_{P}\right)$ that satisfy the equation

$$
D \bar{\partial}_{J, A_{0}}\left(u_{0}\right) \xi_{0}+X_{\alpha_{0}}\left(u_{0}\right)^{0,1}=0 .
$$

This space can be identified with the kernel of the operator $\mathcal{D}_{\left(u_{0}, A_{0}\right)}^{0}$. Namely, the kernel of $\mathcal{D}_{\left(u_{0}, A_{0}\right)}^{0}$ consists of all sections $\xi_{0} \in \Omega^{0}\left(\Sigma, H_{u_{0}}\right)$ that satisfy 
equation (8.9) for some 1-form $\alpha_{0} \in \Omega^{1}\left(\Sigma, \mathfrak{g}_{P}\right)$, and the 1-form $\alpha_{0}$ is uniquely determined by $\xi_{0}$. Thus $\mathcal{V}^{0} \longrightarrow \widetilde{\mathcal{M}}_{B, \Sigma}^{0}$ is a vector bundle of rank $m:=$ $\operatorname{dim} \mathcal{M}_{B, \Sigma}^{0}$.

Lemma 8.4. For every $p>2$ and every $c_{0}>0$, there exist positive constants $\varepsilon_{0}$ and $c$ such that the following holds for every $\varepsilon \in\left(0, \varepsilon_{0}\right]$. Let $I \subset \mathbb{R}$ be an interval and $I \rightarrow \widetilde{\mathcal{M}}_{B, \Sigma}^{0}\left(c_{0}\right): r \mapsto\left(u_{0}(r), A_{0}(r)\right)$ be a smooth path such that, for every $r \in I$,

$$
\left\|\partial_{r} u_{0}(r)\right\|_{C^{2}(\Sigma)}+\left\|\partial_{r} A_{0}(r)\right\|_{C^{1}(\Sigma)} \leq c_{0}
$$

Then every smooth vector field $r \mapsto \zeta(r)=(\xi(r), \alpha(r)) \in T_{\left(u_{0}(r), A_{0}(r)\right)} \mathcal{B}$ satisfies the inequality

$$
\left\|\tilde{\nabla}_{r} \mathcal{D}^{\varepsilon} \zeta-\mathcal{D}^{\varepsilon} \tilde{\nabla}_{r} \zeta\right\|_{k, p, \varepsilon} \leq c \varepsilon^{-1}\|\zeta\|_{k+1, p, \varepsilon}
$$

for $k=0,1$, where $\mathcal{D}^{\varepsilon}:=\mathcal{D}_{\left(u_{0}(r), A_{0}(r)\right)}^{\varepsilon}$ and $\tilde{\nabla}_{r} \zeta:=\left(\nabla_{r} \xi-1 / 2 J\left(\nabla_{\partial_{r} u_{0}} J\right)\right.$ $\left.\xi, \partial_{r} \alpha\right)$.

Proof. We denote

$$
(\xi, \alpha):=\zeta, \quad \zeta^{\prime}:=\left(\xi^{\prime}, \varphi^{\prime}, \psi^{\prime}\right):=\mathcal{D}^{\varepsilon} \zeta, \quad \zeta_{r}^{\prime}:=\left(\xi_{r}^{\prime}, \varphi_{r}^{\prime}, \psi_{r}^{\prime}\right):=\mathcal{D}^{\varepsilon} \tilde{\nabla}_{r} \zeta .
$$

Moreover, we drop the subscript 0 and write $(u, A):=\left(u_{0}, A_{0}\right)$. Then, in local holomorphic coordinates on $\Sigma$ and a local frame of $P, \zeta^{\prime}$ is given by

$$
\begin{aligned}
\xi^{\prime} & =\tilde{\nabla}_{A, s} \xi+J \tilde{\nabla}_{A, t} \xi+\frac{1}{4} N\left(\xi, v_{s}-J v_{t}\right)+\frac{1}{2}\left(J \partial_{s} J-\partial_{t} J\right) \xi+L_{u} \varphi+J L_{u} \psi, \\
\varphi^{\prime} & =\lambda^{-2}\left(\nabla_{A, s} \varphi+\nabla_{A, t} \psi\right)+\varepsilon^{-2} L_{u}^{*} \xi \\
\psi^{\prime} & =\lambda^{-2}\left(\nabla_{A, s} \psi-\nabla_{A, t} \varphi\right)+\varepsilon^{-2} d \mu(u) \xi
\end{aligned}
$$

Here we use the notation of Remark 4.1. Differentiating these formulae with respect to $r$, we obtain

$$
\begin{aligned}
\tilde{\nabla}_{r} \xi^{\prime}-\xi_{r}^{\prime}= & \tilde{\nabla}_{r} \tilde{\nabla}_{A, s} \xi-\tilde{\nabla}_{A, s} \tilde{\nabla}_{r} \xi+J\left(\tilde{\nabla}_{r} \tilde{\nabla}_{A, t} \xi-\tilde{\nabla}_{A, t} \tilde{\nabla}_{r} \xi\right) \\
& +\frac{1}{4} \tilde{\nabla}_{r} N\left(\xi, v_{s}-J v_{t}\right)-\frac{1}{4} N\left(\tilde{\nabla}_{r} \xi, v_{s}-J v_{t}\right) \\
& +\frac{1}{2} \tilde{\nabla}_{r}\left(\left(J \partial_{s} J-\partial_{t} J\right) \xi\right)-\frac{1}{2}\left(J \partial_{s} J-\partial_{t} J\right) \tilde{\nabla}_{r} \xi \\
& +\nabla_{\partial_{r} u} X_{\varphi}(u)+J \nabla_{\partial_{r} u} X_{\psi}(u)-\frac{1}{2} J\left(\nabla_{\partial_{r} u} J\right)\left(L_{u} \varphi-J L_{u} \psi\right), \\
\partial_{r} \varphi^{\prime}-\varphi_{r}^{\prime}= & \lambda^{-2}\left(\left[\partial_{r} \Phi, \varphi\right]+\left[\partial_{r} \Psi, \psi\right]\right) \\
& +\varepsilon^{-2}\left(\rho\left(\partial_{r} u, \xi\right)-\frac{1}{2} d \mu(u)\left(\nabla_{\partial_{r} u} J\right) \xi\right), \\
\partial_{r} \psi^{\prime}-\psi_{r}^{\prime}= & \lambda^{-2}\left(\left[\partial_{r} \Phi, \psi\right]-\left[\partial_{r} \Psi, \varphi\right]\right) \\
& -\varepsilon^{-2}\left(\rho\left(\partial_{r} u, J \xi\right)+\frac{1}{2} L_{u}^{*}\left(\nabla_{\partial_{r} u} J\right) \xi\right) .
\end{aligned}
$$


Here $\rho=\rho_{s, t} \in \Omega^{2}(M, \mathfrak{g})$ is defined by Lemma C.2. The required estimates follow from these three identities via a term-by-term inspection.

Lemma 8.5. For every $p>2$ and every $c_{0}>0$, there exist positive constants $\varepsilon_{0}$ and $c$ such that the following holds for every $\varepsilon \in\left(0, \varepsilon_{0}\right]$. Let $r \mapsto\left(u_{0}(r), A_{0}(r)\right)$ be as in Lemma 8.4 and suppose that $r \mapsto \zeta_{0}(r)$ is a smooth section of $\mathcal{V}^{0}$ along this path. Abbreviate $\mathcal{D}^{\varepsilon}:=\mathcal{D}_{\left(u_{0}(r), A_{0}(r)\right)}^{\varepsilon}$ and let $\zeta_{\varepsilon}(r) \in \operatorname{ker} \mathcal{D}^{\varepsilon}$ be given by

$$
\zeta_{\varepsilon}(r):=\zeta_{0}(r)-\mathcal{D}^{\varepsilon *}\left(\mathcal{D}^{\varepsilon} \mathcal{D}^{\varepsilon *}\right)^{-1} \mathcal{D}^{\varepsilon} \zeta_{0}(r)
$$

Then

$$
\begin{aligned}
\left\|\zeta_{\varepsilon}-\zeta_{0}\right\|_{0,2, \varepsilon} & \leq c \varepsilon^{2}\left\|\xi_{0}\right\|_{L^{2}} \\
\left\|\tilde{\nabla}_{r} \zeta_{\varepsilon}-\tilde{\nabla}_{r} \zeta_{0}\right\|_{0,2, \varepsilon} & \leq c\left(\left\|\xi_{0}\right\|_{L^{2}}+\left\|\tilde{\nabla}_{r} \xi_{0}\right\|_{L^{2}}\right) .
\end{aligned}
$$

Proof. Let $\left(\xi_{\varepsilon}, \alpha_{\varepsilon}\right):=\zeta_{\varepsilon}$ for $\varepsilon \geq 0$ and $\zeta:=(\xi, \alpha):=\zeta_{\varepsilon}-\zeta_{0}$. Then

$$
\zeta \in \operatorname{im} \mathcal{D}^{\varepsilon *}, \quad \mathcal{D}^{\varepsilon} \zeta=\left(\begin{array}{c}
0 \\
d_{A_{0}}^{*} \alpha_{0} \\
-* d_{A_{0}} \alpha_{0}
\end{array}\right) .
$$

Hence, by Lemma 4.8, there exist constants $c_{1}, c_{2}>0$ (depending only on $c_{0}$ ) such that

$$
\|\zeta\|_{1,2, \varepsilon} \leq c_{1} \varepsilon\left\|\mathcal{D}^{\varepsilon} \zeta\right\|_{0,2, \varepsilon}=c_{1} \varepsilon^{2}\left(\left\|d_{A_{0}} \alpha_{0}\right\|_{L^{2}}+\left\|d_{A_{0}}^{*} \alpha_{0}\right\|_{L^{2}}\right) \leq c_{2} \varepsilon^{2}\left\|\xi_{0}\right\|_{L^{2}} .
$$

The last inequality follows from equation (8.9) and the basic elliptic estimates for the operator $\mathcal{D}^{0}$. Thus we have proved equation (8.11). To prove equation (8.12), let

$$
\zeta^{\prime}:=\zeta^{\prime}(r):=-\left(\mathcal{D}^{\varepsilon} \mathcal{D}^{\varepsilon *}\right)^{-1} \mathcal{D}^{\varepsilon} \zeta_{0}(r) \in \mathcal{X}_{u_{0}(r)}^{\prime}
$$

so that $\zeta=\mathcal{D}^{\varepsilon *} \zeta^{\prime}$. Then, by Lemma 4.7 with $p=2$,

$$
\left\|\zeta^{\prime}\right\|_{2,2, \varepsilon} \leq c_{3}\left\|\mathcal{D}^{\varepsilon *} \zeta^{\prime}\right\|_{1,2, \varepsilon}=c_{3}\|\zeta\|_{1,2, \varepsilon} \leq c_{2} c_{3} \varepsilon^{2}\left\|\xi_{0}\right\|_{L^{2}}
$$

and hence, by Lemma 8.4 (with $c=c_{4}$ and $\mathcal{D}^{\varepsilon}$ replaced by $\mathcal{D}^{\varepsilon *}$ ),

$$
\left\|\mathcal{D}^{\varepsilon *} \tilde{\nabla}_{r} \zeta^{\prime}-\tilde{\nabla}_{r} \mathcal{D}^{\varepsilon *} \zeta^{\prime}\right\|_{1,2, \varepsilon} \leq c_{4} \varepsilon^{-1}\left\|\zeta^{\prime}\right\|_{2,2, \varepsilon} \leq c_{2} c_{3} c_{4} \varepsilon\left\|\xi_{0}\right\|_{L^{2}}
$$


Now it follows from Lemmas 4.8 (with $c=c_{5}$ ) and 8.4 (with $c=c_{4}$ ) that

$$
\begin{aligned}
\left\|\mathcal{D}^{\varepsilon *} \tilde{\nabla}_{r} \zeta^{\prime}\right\|_{1,2, \varepsilon} \leq & c_{5}\left\|\mathcal{D}^{\varepsilon} \mathcal{D}^{\varepsilon *} \tilde{\nabla}_{r} \zeta^{\prime}\right\|_{0,2, \varepsilon} \\
\leq & c_{5}\left\|\mathcal{D}^{\varepsilon}\left(\mathcal{D}^{\varepsilon *} \tilde{\nabla}_{r} \zeta^{\prime}-\tilde{\nabla}_{r} \mathcal{D}^{\varepsilon *} \zeta^{\prime}\right)\right\|_{0,2, \varepsilon} \\
& +c_{5}\left\|\mathcal{D}^{\varepsilon} \tilde{\nabla}_{r} \zeta-\tilde{\nabla}_{r} \mathcal{D}^{\varepsilon} \zeta\right\|_{0,2, \varepsilon}+c_{5}\left\|\tilde{\nabla}_{r} \mathcal{D}^{\varepsilon} \zeta\right\|_{0,2, \varepsilon} \\
\leq & c_{6} \varepsilon^{-1}\left\|\mathcal{D}^{\varepsilon *} \tilde{\nabla}_{r} \zeta^{\prime}-\tilde{\nabla}_{r} \mathcal{D}^{\varepsilon *} \zeta^{\prime}\right\|_{1,2, \varepsilon} \\
& +c_{5}\left(c_{4} \varepsilon^{-1}\|\zeta\|_{1,2, \varepsilon}+\left\|\tilde{\nabla}_{r} \mathcal{D}^{\varepsilon} \zeta\right\|_{0,2, \varepsilon}\right) \\
\leq & c_{7}\left(\left\|\xi_{0}\right\|_{L^{2}}+\left\|\tilde{\nabla}_{r} \xi_{0}\right\|_{L^{2}}\right)
\end{aligned}
$$

Hence

$$
\begin{aligned}
\left\|\tilde{\nabla}_{r} \zeta\right\|_{0,2, \varepsilon} & \leq\left\|\tilde{\nabla}_{r} \mathcal{D}^{\varepsilon *} \zeta^{\prime}-\mathcal{D}^{\varepsilon *} \tilde{\nabla}_{r} \zeta^{\prime}\right\|_{0,2, \varepsilon}+\left\|\mathcal{D}^{\varepsilon *} \tilde{\nabla}_{r} \zeta^{\prime}\right\|_{0,2, \varepsilon} \\
& \leq\left(c_{7}+c_{2} c_{3} c_{4} \varepsilon\right)\left(\left\|\xi_{0}\right\|_{L^{2}}+\left\|\tilde{\nabla}_{r} \xi_{0}\right\|_{L^{2}}\right)
\end{aligned}
$$

This proves equation (8.12).

The estimate (8.12) is fairly crude. More careful considerations give an additional factor $\varepsilon$. However, we shall not use this fact.

Lemma 8.6. For every $p>2$ and every $c_{0}>0$, there exist positive constants $\delta, \varepsilon_{0}$, and $c$ such that the following holds for every $\varepsilon \in\left(0, \varepsilon_{0}\right]$. Let $(u, A) \in \mathcal{B}$ and $I \subset \mathbb{R}$ be an interval. Suppose that the path $I \rightarrow \mathcal{U}^{0}(\delta, \varepsilon)$ : $r \mapsto\left(u_{0}(r), A_{0}(r)\right)$ and the vector field $r \mapsto \zeta(r) \in T_{\left(u_{0}(r), A_{0}(r)\right)} \mathcal{B}$ are as in Lemma 8.3. Moreover, assume equation (8.10) and let $r \mapsto \zeta_{0}(r)$ and $r \mapsto \zeta_{\varepsilon}(r)$ be as in Lemma 8.5. Then

$$
\begin{aligned}
& \left|\frac{\mathrm{d}}{\mathrm{d} r}\left\langle\zeta_{\varepsilon}, \mathcal{S}^{\varepsilon}\left(u_{0}, A_{0}\right)\right\rangle_{\varepsilon}+\left\langle\xi_{0}, \dot{u}_{0}\right\rangle\right| \\
& \quad \leq c\left(\left\|\xi_{0}\right\|_{L^{2}}+\left\|\tilde{\nabla}_{r} \xi_{0}\right\|_{L^{2}}\right)\left(\varepsilon^{2}+\|\zeta\|_{1, p, \varepsilon}+\left\|L_{u_{0}}^{*} \partial_{r} u_{0}\right\|_{L^{p}}\right) .
\end{aligned}
$$

Proof. Abbreviate $\mathcal{S}^{\varepsilon}:=\mathcal{S}^{\varepsilon}\left(u_{0}, A_{0}\right)$. Consider the identity

$$
\begin{aligned}
\frac{\mathrm{d}}{\mathrm{d} r}\left\langle\zeta_{\varepsilon}, \mathcal{S}^{\varepsilon}\right\rangle_{\varepsilon}+\left\langle\xi_{0}, \partial_{r} u_{0}\right\rangle_{\varepsilon}= & \left\langle\tilde{\nabla}_{r} \zeta_{\varepsilon}, \mathcal{S}^{\varepsilon}\right\rangle_{\varepsilon}+\left\langle\zeta_{\varepsilon},\left(\partial_{r} u_{0}, \partial_{r} A_{0}\right)+\tilde{\nabla}_{r} \mathcal{S}^{\varepsilon}\right\rangle_{\varepsilon} \\
& +\left\langle\zeta_{0}-\zeta_{\varepsilon},\left(\partial_{r} u_{0}, \partial_{r} A_{0}\right)\right\rangle_{\varepsilon}-\varepsilon^{2}\left\langle\alpha_{0}, \partial_{r} A_{0}\right\rangle .
\end{aligned}
$$

By Lemma 8.5 , the $(0,2, \varepsilon)$-norm of $\tilde{\nabla}_{r} \zeta_{\varepsilon}$ is bounded above by a constant times $\left\|\xi_{0}\right\|_{L^{2}}+\left\|\tilde{\nabla}_{r} \xi_{0}\right\|_{L^{2}}$. By Proposition 7.1 , the $(0,2, \varepsilon)$-norm of $\mathcal{S}^{\varepsilon}$ is bounded above by a constant times $\|\zeta\|_{1, p, \varepsilon}$. Hence the first term satisfies the required bound. For the second term, the estimate follows from Lemma 8.3 and the fact that the $(0,2, \varepsilon)$-norm of $\zeta_{\varepsilon}$ is bounded above by a constant times $\left\|\xi_{0}\right\|_{L^{2}}$. For the third term, we use equations (8.10) and (8.11) and for the last the estimate follows from equation (8.10). 
Proof of Proposition 8.2. Let $U \subset \mathbb{R}^{m}$ be an open set containing zero and

$$
U \longrightarrow \widetilde{\mathcal{M}}_{B, \Sigma}^{0}\left(c_{0}\right): x \mapsto\left(u_{0}(x), A_{0}(x)\right)
$$

be the composition of the map $\mathcal{F}_{\left(\bar{u}_{0}, \bar{A}_{0}\right)}^{0}$ defined in Theorem 2.1 with a Hilbert space isomorphism $\mathbb{R}^{m} \rightarrow \operatorname{ker} \mathcal{D}_{\left(\bar{u}_{0}, \bar{A}_{0}\right)}^{0}$. Then

$$
\left(u_{0}(0), A_{0}(0)\right)=\left(\bar{u}_{0}, \bar{A}_{0}\right), \quad\left(\partial_{i} u_{0}(0), \partial_{i} A_{0}(0)\right) \in \mathcal{V}_{\left(\bar{u}_{0}, \bar{A}_{0}\right)}^{0}
$$

for $i=1, \ldots, m$; in particular,

$$
L_{\bar{u}_{0}}^{*} \partial_{i} u_{0}(0)=0, \quad\left\langle\partial_{i} u_{0}(0), \partial_{j} u_{0}(0)\right\rangle_{L^{2}(\Sigma)}=\delta_{i j} .
$$

Now choose $m$ smooth sections $\zeta_{10}, \ldots, \zeta_{m 0}: U \rightarrow \mathcal{V}^{0}$ so that

$$
\zeta_{j 0}(x)=\left(\xi_{j 0}(x), \alpha_{j 0}(x)\right) \in \mathcal{V}_{\left(u_{0}(x), A_{0}(x)\right)}^{0},
$$

and

$$
\zeta_{j 0}(0)=-\left(\partial_{j} u_{0}(0), \partial_{j} A_{0}(0)\right), \quad\left\langle\xi_{i 0}(x), \xi_{j 0}(x)\right\rangle_{L^{2}(\Sigma)}=\delta_{i j}
$$

for $x \in U$ and $j=1, \ldots, m$. Given $x \in U$ we abbreviate $\mathcal{D}^{\varepsilon}:=\mathcal{D}_{\left(u_{0}(x), A_{0}(x)\right)}^{\varepsilon}$. If $\varepsilon$ is sufficiently small then, by Lemma 4.8, this operator is surjective for every $x \in U$. In this case, we define $\zeta_{j \varepsilon}(x) \in \operatorname{ker} \mathcal{D}^{\varepsilon}$ by

$$
\zeta_{j \varepsilon}(x):=\zeta_{j 0}(x)-\mathcal{D}^{\varepsilon *}\left(\mathcal{D}^{\varepsilon} \mathcal{D}^{\varepsilon *}\right)^{-1} \mathcal{D}^{\varepsilon} \zeta_{j 0}(x)
$$

for $j=1, \ldots, m$. By Lemma 8.5, these vectors form a basis of ker $\mathcal{D}^{\varepsilon}$ for $\varepsilon$ sufficiently small. Now let $\delta_{1}$ and $c_{1}$ be the constants of Proposition 7.1. Choose $\delta_{0}>0$ so small that

$$
|x|<\delta_{0} \varepsilon^{2 / p} \quad \Longrightarrow \quad\left(u_{0}(x), A_{0}(x)\right) \in \mathcal{U}^{0}\left(\delta_{1}, \varepsilon\right)
$$

for $x \in U$ and $0<\varepsilon \leq 1$. Let $\mathcal{S}^{\varepsilon}: \mathcal{U}^{0} \rightarrow \mathcal{H}^{\varepsilon}$ be the map of Proposition 7.1 as introduced above. Define $\theta=\left(\theta_{1}, \ldots, \theta_{m}\right): U \rightarrow \mathbb{R}^{m}$ by

$$
\theta_{j}(x):=\left\langle\zeta_{j \varepsilon}(x), \mathcal{S}^{\varepsilon}\left(u_{0}(x), A_{0}(x)\right)\right\rangle_{\varepsilon},
$$

where $\langle\cdot, \cdot\rangle_{\varepsilon}$ denotes the $(0,2, \varepsilon)$-inner product on $T_{\left(u_{0}(x), A_{0}(x)\right)} \mathcal{B}$. Then

$$
\theta(x)=0 \quad \Longleftrightarrow \quad \mathcal{S}^{\varepsilon}\left(u_{0}(x), A_{0}(x)\right) \in \operatorname{im} \mathcal{D}^{\varepsilon *} .
$$

We shall establish the existence of a zero of $\theta$ with the inverse function theorem. We must prove that $|d \theta(x)-\mathbb{1}| \leq 1 / 2$ on a ball of radius $r$ and that $\theta(0)$ is less than $r / 2$.

To see this, we first observe that

$$
\left|\theta_{j}(0)\right| \leq\left\|\zeta_{j 0}(0)\right\|_{0,2, \varepsilon}\left\|\mathcal{S}^{\varepsilon}\left(\bar{u}_{0}, \bar{A}_{0}\right)\right\|_{0,2, \varepsilon} \leq c_{2}\|\bar{\zeta}\|_{1, p, \varepsilon} \leq c_{2} \delta \varepsilon^{2 / p}
$$

Here we have used the fact that the $(0,2, \varepsilon)$-norm of $\zeta_{j \varepsilon}(0)$ is less than or equal to the $(0,2, \varepsilon)$-norm of $\zeta_{j 0}(0)=-\left(\partial_{j} u_{0}(0), \partial_{j} A_{0}(0)\right)$, that the $L^{2}$ norm of $\partial_{j} A_{0}(0)$ is controlled by $\left\|\partial_{j} u_{0}(0)\right\|_{L^{2}}=1$, that the $(0,2, \varepsilon)$-norm of $\mathcal{S}^{\varepsilon}\left(\bar{u}_{0}, \bar{A}_{0}\right)$ is controlled by its $(1, p, \varepsilon)$-norm, and that, by Proposition 7.1 
and equation (8.1), the latter is bounded above by $c_{1}\|\bar{\zeta}\|_{1, p, \varepsilon} \leq c_{1} \delta \varepsilon^{2 / p}$. Thus we have proved that

$$
|\theta(0)| \leq \sqrt{m} c_{2}\|\bar{\zeta}\|_{1, p, \varepsilon} \leq \sqrt{m} c_{2} \delta \varepsilon^{2 / p} .
$$

Now let $\zeta(x)=(\xi(x), \alpha(x)) \in T_{\left(u_{0}(x), A_{0}(x)\right)} \mathcal{B}$ be the unique smooth section defined by

$$
(u, A)=\left(\exp _{u_{0}(x)}(\xi(x)), A_{0}(x)+\alpha(x)\right), \quad \zeta(0)=\bar{\zeta},
$$

for $x$ sufficiently small. Then there exists a constant $c_{3}>0$ such that

$$
\|\zeta(x)\|_{1, p, \varepsilon} \leq\|\bar{\zeta}\|_{1, p, \varepsilon}+c_{3}|x|
$$

for $x$ sufficiently small and $0<\varepsilon \leq 1$. Hence, by Proposition 7.1, we have that, for $|x|<\delta_{0} \varepsilon^{2 / p}$ and $0<\varepsilon \leq 1$,

$$
\begin{aligned}
& \left\|\mathcal{N}^{\varepsilon}\left(u_{0}(x), A_{0}(x)\right)\right\|_{2, p, \varepsilon}+\left\|\mathcal{S}^{\varepsilon}\left(u_{0}(x), A_{0}(x)\right)\right\|_{1, p, \varepsilon} \\
& \quad \leq c_{1}\|\zeta(x)\|_{1, p, \varepsilon} \leq c_{1}\left(\|\bar{\zeta}\|_{1, p, \varepsilon}+c_{3}|x|\right) .
\end{aligned}
$$

Moreover, there exists a constant $c_{4}>0$ such that

$$
\begin{gathered}
\left|\delta_{i j}+\left\langle\xi_{j 0}(x), \partial_{i} u_{0}(x)\right\rangle_{L^{2}}\right|+\left\|L_{u_{0}(x)}^{*} \partial_{i} u_{0}(x)\right\|_{L^{p}} \leq c_{4}|x|, \\
\left\|\xi_{j 0}(x)\right\|_{L^{2}}+\left\|\tilde{\nabla}_{i} \xi_{j 0}(x)\right\|_{L^{2}} \leq c_{4},
\end{gathered}
$$

for $x$ sufficiently small. Now suppose that $\delta_{1}$ and $\varepsilon_{0}$ have been chosen so small that the assertion of Lemma 8.6 holds, with $c$ replaced by $c_{5}$, for the paths $x_{i} \mapsto\left(u_{0}(x), A_{0}(x)\right), x_{i} \mapsto \zeta(x), x_{i} \mapsto \zeta_{j 0}(x)$, and $x_{i} \mapsto \zeta_{j \varepsilon}(x)$. Then

$$
\begin{aligned}
\left|\partial_{i} \theta_{j}(x)-\delta_{i j}\right| \leq & \left|\delta_{i j}+\left\langle\xi_{j 0}(x), \partial_{i} u_{0}(x)\right\rangle\right|+\left|\partial_{i} \theta_{j}(x)+\left\langle\xi_{j 0}(x), \partial_{i} u_{0}(x)\right\rangle\right| \\
\leq & c_{4}|x|+c_{5}\left(\left\|\xi_{j 0}(x)\right\|_{L^{2}}+\left\|\tilde{\nabla}_{i} \xi_{j 0}(x)\right\|_{L^{2}}\right) \\
& \times\left(\varepsilon^{2}+\|\zeta(x)\|_{1, p, \varepsilon}+\left\|L_{u_{0}(x)}^{*} \partial_{i} u_{0}(x)\right\|_{L^{p}}\right) \\
\leq & c_{4}|x|+c_{4} c_{5}\left(\varepsilon^{2}+\|\bar{\zeta}\|_{1, p, \varepsilon}+\left(c_{3}+c_{4}\right)|x|\right) \\
\leq & c_{6}\left(\varepsilon^{2}+\|\bar{\zeta}\|_{1, p, \varepsilon}+|x|\right)
\end{aligned}
$$

for $|x|<\delta_{0} \varepsilon^{2 / p}$ and $0<\varepsilon \leq \varepsilon_{0}$. Thus the Jacobian $d \theta(x) \in \mathbb{R}^{m \times m}$ satisfies

$$
|d \theta(x)-\mathbb{1}| \leq c_{7}\left(\varepsilon^{2}+\delta \varepsilon^{2 / p}+|x|\right) .
$$

Choose $\delta_{0}$ and $\varepsilon_{0}$ so small that $c_{7}\left(\varepsilon_{0}^{2}+2 \delta_{0} \varepsilon_{0}^{2 / p}\right) \leq 1 / 2$. Then

$$
|x| \leq \delta_{0} \varepsilon^{2 / p}, \quad 0<\varepsilon \leq \varepsilon_{0}, \quad 0<\delta \leq \delta_{0} \quad \Longrightarrow \quad|d \theta(x)-11| \leq \frac{1}{2} .
$$

Hence the inverse function theorem asserts that $\theta\left(B_{r}(0)\right) \supset B_{r / 2}(\theta(0))$ whenever $r<\delta_{0} \varepsilon^{2 / p}$. Now suppose that $\sqrt{m} c_{2} \delta<\delta_{0} / 2$. Then, by equation (8.13), 
we have $2|\theta(0)|<\delta_{0} \varepsilon^{2 / p}$ and hence we can apply the inverse function theorem with $r=2|\theta(0)|$. Then $B_{r / 2}(\theta(0))$ contains zero and, by the inverse function theorem, there exists a point $x_{0} \in \mathbb{R}^{m}$ such that

$$
\theta\left(x_{0}\right)=0, \quad\left|x_{0}\right| \leq 2|\theta(0)| \leq 2 \sqrt{m} c_{2}\|\bar{\zeta}\|_{1, p, \varepsilon} .
$$

The last inequality follows from equation (8.13). Now define

$$
\left(u_{0}, A_{0}\right):=\left(u_{0}\left(x_{0}\right), A_{0}\left(x_{0}\right)\right), \quad \zeta_{0}:=\mathcal{S}^{\varepsilon}\left(u_{0}, A_{0}\right), \quad \eta_{0}:=\mathcal{N}^{\varepsilon}\left(u_{0}, A_{0}\right) .
$$

Then equations (8.2) and (8.3) are satisfied by definition. The estimate (8.4) follows from Proposition 7.1:

$$
\left\|\eta_{0}\right\|_{2, p, \varepsilon}+\left\|\zeta_{0}\right\|_{1, p, \varepsilon} \leq c_{1}\left\|\zeta\left(x_{0}\right)\right\|_{1, p, \varepsilon} \leq c_{1}\left(\|\bar{\zeta}\|_{1, p, \varepsilon}+c_{3}\left|x_{0}\right|\right) \leq c_{8}\|\bar{\zeta}\|_{1, p, \varepsilon} .
$$

Moreover, the vector $\bar{\xi}_{0}$ in the assertion of Proposition 8.2 is the image of $x_{0}$ under our Hilbert space isomorphism $\mathbb{R}^{m} \rightarrow \operatorname{ker} \mathcal{D}_{\left(\bar{u}_{0}, \bar{A}_{0}\right)}^{0}$. Hence, by elliptic regularity for the Cauchy-Riemann operator, its $W^{1, p}$-norm is bounded by $\left|x_{0}\right|$ and hence by the $(1, p, \varepsilon)$-norm of $\bar{\zeta}$.

Proof of Theorem 8.1. Let $p>2$ and $c_{0}>0$ be given. Choose positive constants $\varepsilon_{0}, \delta_{1}, \delta_{2}$, and $c_{1}$ such that Proposition 8.2 holds with $\delta$ replaced by $\delta_{1}$ and $c$ replaced by $c_{1}$, Corollary 6.3 holds with $\delta$ replaced by $\delta_{2}$, and both results hold for $0<\varepsilon \leq \varepsilon_{0}$. Now choose $\delta>0$ so small that

$$
\delta \varepsilon_{0}{ }^{1 / 2} \leq \delta_{1}, \quad c_{1} \delta \leq \delta_{2} .
$$

Let $\varepsilon \in\left(0, \varepsilon_{0}\right]$ and suppose that $(u, A)$ and $\left(\bar{u}_{0}, \bar{A}_{0}\right)$ satisfy the hypotheses of Theorem 3.1, namely

$$
\left(\bar{u}_{0}, \bar{A}_{0}\right) \in \widetilde{\mathcal{M}}_{B, \Sigma}^{0}\left(c_{0}-1\right), \quad(u, A)=\left(\exp _{\bar{u}_{0}}(\bar{\xi}), \bar{A}_{0}+\bar{\alpha}\right) \in \widetilde{\mathcal{M}}_{B, \Sigma}^{\varepsilon},
$$

where $\bar{\zeta}:=(\bar{\xi}, \bar{\alpha}) \in T_{\left(\bar{u}_{0}, \bar{A}_{0}\right)} \mathcal{B}$ satisfies

$$
\|\bar{\zeta}\|_{1, p, \varepsilon ;\left(\bar{u}_{0}, \bar{A}_{0}\right)} \leq \delta \varepsilon^{2 / p+1 / 2} \leq \delta_{1} \varepsilon^{2 / p} .
$$

By Proposition 8.2, there exist

$$
\bar{\xi}_{0} \in \operatorname{ker} \mathcal{D}_{\left(\bar{u}_{0}, \bar{A}_{0}\right)}^{0}, \quad \eta_{0} \in \Omega^{0}\left(\Sigma, \mathfrak{g}_{P}\right)
$$

and

$$
\left(u_{0}, A_{0}\right) \in \widetilde{\mathcal{M}}_{B, \Sigma}^{0}\left(c_{0}\right), \quad \zeta_{0}=\left(\xi_{0}, \alpha_{0}\right) \in T_{\left(u_{0}, A_{0}\right)} \mathcal{B},
$$

satisfying equations (8.2) to (8.4), with $c$ replaced by $c_{1}$. Hence

$$
\left\|\zeta_{0}\right\|_{1, p, \varepsilon ;\left(u_{0}, A_{0}\right)} \leq c_{1}\|\bar{\zeta}\|_{\left.1, p, \varepsilon ; \bar{u}_{0}, \bar{A}_{0}\right)} \leq c_{1} \delta \varepsilon^{2 / p+1 / 2} \leq \delta_{2} \varepsilon^{2 / p+1 / 2} .
$$

This estimate together with equation (8.3) shows that $\left(u_{0}, A_{0}\right)$ and $\zeta_{0}$ satisfy the hypotheses of Corollary 6.3. Hence, by equation (8.2),

$$
g^{*}(u, A)=\left(\exp _{u_{0}}\left(\xi_{0}\right), A_{0}+\alpha_{0}\right)=\widetilde{\mathcal{T}}^{\varepsilon}\left(u_{0}, A_{0}\right), \quad g:=e^{\eta_{0}} .
$$


Moreover, again by equation $(8.2),\left(u_{0}, A_{0}\right)=\mathcal{F}_{\left(\bar{u}_{0}, \bar{A}_{0}\right)}^{0}\left(\bar{\xi}_{0}\right)$ and, by equation (8.4),

$$
\left\|\bar{\zeta}_{0}\right\|_{W^{1, p}}+\left\|\eta_{0}\right\|_{2, p, \varepsilon ; \bar{A}_{0}} \leq c_{1}\|\bar{\zeta}\|_{1, p, \varepsilon ;\left(\bar{u}_{0}, \bar{A}_{0}\right)} .
$$

This proves the theorem.

\section{A priori estimates}

In this section, we assume that $J \in \mathcal{J}_{\mathrm{G}}(M, \omega)$ is independent of $z \in \Sigma$ (or in local coordinates is independent of $s$ and $t$ ). Let $\Omega \subset \mathbb{C}$ be an open set, $K \subset \Omega$ be a compact subset and $\lambda: \Omega \rightarrow(0, \infty)$ be a smooth function. Given $u: \Omega \rightarrow M$ and $\Phi, \Psi: \Omega \rightarrow \mathfrak{g}$, we define $v_{s}, v_{t}: \Omega \rightarrow u^{*} T M$ and $\kappa: \Omega \rightarrow \mathfrak{g}$ by

$$
v_{s}:=\partial_{s} u+X_{\Phi}(u), \quad v_{t}:=\partial_{t} u+X_{\Psi}(u), \quad \kappa:=\partial_{s} \Psi-\partial_{t} \Phi+[\Phi, \Psi] .
$$

Moreover, as in Remark 4.1, we use the notation

$$
\begin{array}{rlrlrl}
\nabla_{A, s} \xi & := & \nabla_{s} \xi+\nabla_{\xi} X_{\Phi}(u), & \nabla_{A, t} \xi & := & \nabla_{t} \xi+\nabla_{\xi} X_{\Psi}(u), \\
\nabla_{A, s} \eta & :=\partial_{s} \eta+[\Phi, \eta], & \nabla_{A, t} \eta & := & \partial_{t} \eta+[\Psi, \eta],
\end{array}
$$

for $\xi: \Omega \rightarrow u^{*} T M$ and $\eta: \Omega \rightarrow \mathfrak{g}$. Then

$$
\nabla_{A, s} \mu(u)=d \mu(u) v_{s}=-L_{u}^{*} J v_{s}, \quad \nabla_{A, t} \mu(u)=d \mu(u) v_{t}=-L_{u}^{*} J v_{t} .
$$

Moreover,

by Lemma C.3,

$$
\nabla_{A, s} v_{t}-\nabla_{A, t} v_{s}=L_{u} \kappa
$$

$$
\nabla_{A, s} L_{u} \eta-L_{u} \nabla_{A, s} \eta=\nabla_{v_{s}} X_{\eta}(u), \quad \nabla_{A, t} L_{u} \eta-L_{u} \nabla_{A, t} \eta=\nabla_{v_{t}} X_{\eta}(u),
$$

and, by Lemma B.4,

$$
\nabla_{A, s} \nabla_{A, t} \xi-\nabla_{A, t} \nabla_{A, s} \xi=R\left(v_{s}, v_{t}\right) \xi+\nabla_{\xi} X_{\kappa}(u)
$$

In local coordinates, equations (1.7) have the form

$$
v_{s}+J v_{t}=0, \quad \lambda^{-2} \kappa+\varepsilon^{-2} \mu(u)=0 .
$$

If the above equation holds then

$$
\left(\nabla_{v_{s}} J\right) v_{t}=\left(\nabla_{v_{t}} J\right) v_{s}, \quad \nabla_{A, s} v_{s}+\nabla_{A, t} v_{t}=-J L_{u} \kappa .
$$

Given a constant $C>0$ we denote by $M^{C} \subset M$ the compact subset of all $x \in M$ that satisfy $|\mu(x)| \leq C$ and $|\eta| \leq C\left|L_{x} \eta\right|$ for every $\eta \in \mathfrak{g}$.

Lemma 9.1. Let $C>0, p \geq 2, \Omega \subset \mathbb{C}$ be an open set, and $K \subset \Omega$ be a compact subset. Then there are positive constants $\varepsilon_{0}$ and $c$ such that the following holds. If $0<\varepsilon \leq \varepsilon_{0}, \lambda: \Omega \rightarrow \mathbb{R}$ is a smooth function satisfying $\lambda \geq 1 / C$, and $(u, \Phi, \Psi)$ is a solution of (9.1) satisfying

$$
\left\|v_{s}\right\|_{L^{\infty}(\Omega)} \leq C, \quad u(\Omega) \subset M^{C},
$$


then

$$
\int_{K}|\mu(u)|^{p} \leq c \varepsilon^{2 p}, \quad \sup _{K}|\mu(u)| \leq c \varepsilon^{2-2 / p} .
$$

Proof. Let $\Delta=\partial^{2} / \partial s^{2}+\partial^{2} / \partial t^{2}$ denote the standard Laplacian. For $r>0$ denote $B_{r}\left(z_{0}\right):=\left\{z \in \mathbb{C}|| z-z_{0} \mid<r\right\}$ and $B_{r}:=B_{r}(0)$. By (9.1), we have

$$
d \mu(u) v_{s}=-L_{u}^{*} J v_{s}=-L_{u}^{*} v_{t}, \quad d \mu(u) v_{t}=-L_{u}^{*} J v_{t}=L_{u}^{*} v_{s},
$$

and hence

$$
\begin{aligned}
\nabla_{A, s} \nabla_{A, s} \mu(u)+\nabla_{A, t} \nabla_{A, t} \mu(u) & =\nabla_{A, s} d \mu(u) v_{s}+\nabla_{A, t} d \mu(u) v_{t} \\
& =\nabla_{A, t} L_{u}^{*} v_{s}-\nabla_{A, s} L_{u}^{*} v_{t} \\
& =L_{u}^{*}\left(\nabla_{A, t} v_{s}-\nabla_{A, s} v_{t}\right)-2 \rho\left(v_{s}, v_{t}\right) \\
& =-L_{u}^{*} L_{u} \kappa-2 \rho\left(v_{s}, v_{t}\right) \\
& =\left(\frac{\lambda}{\varepsilon}\right)^{2} L_{u}^{*} L_{u} \mu(u)-2 \rho\left(v_{s}, v_{t}\right) .
\end{aligned}
$$

Here $\rho \in \Omega^{2}(M, \mathfrak{g})$ is as in Lemma C.2. Thus

$$
\begin{aligned}
\Delta|\mu(u)|^{2}= & 2\left|\nabla_{A, s} \mu(u)\right|^{2}+2\left|\nabla_{A, t} \mu(u)\right|^{2} \\
& +2\left\langle\mu(u), \nabla_{A, s} \nabla_{A, s} \mu(u)+\nabla_{A, t} \nabla_{A, t} \mu(u)\right\rangle \\
= & 2\left|\nabla_{A, s} \mu(u)\right|^{2}+2\left|\nabla_{A, t} \mu(u)\right|^{2} \\
& +2\left(\frac{\lambda}{\varepsilon}\right)^{2}\left|L_{u} \mu(u)\right|^{2}-4\left\langle\mu(u), \rho\left(v_{s}, v_{t}\right)\right\rangle .
\end{aligned}
$$

Now choose positive constants $\delta$ and $c$ such that

$$
2 \min _{\Omega} \lambda^{2} \geq \delta C^{2}, \quad 4\left|\rho\left(\xi_{1}, \xi_{2}\right)\right| \leq \frac{c}{C^{2}}\left|\xi_{1}\right|\left|\xi_{2}\right|,
$$

for all $x \in M^{C}$ and $\xi_{1}, \xi_{2} \in T_{x} M$. Then

$$
\Delta|\mu(u)|^{2} \geq \frac{\delta}{\varepsilon^{2}}|\mu(u)|^{2}-c|\mu(u)| .
$$

Since $2 \Delta|\mu(u)|^{p} \geq p|\mu(u)|^{p-2} \Delta|\mu(u)|^{2}$ for $p \geq 2$, this implies

$$
|\mu(u)|^{p} \leq \frac{c \varepsilon^{2}}{\delta}|\mu(u)|^{p-1}+\frac{2 \varepsilon^{2}}{p \delta} \Delta|\mu(u)|^{p} .
$$

Using the inequality $a b \leq a^{p} / p+b^{q} / q$ with $1 / p+1 / q=1, a:=c \varepsilon^{2} / \delta$ and $b:=|\mu(u)|^{p-1}$ we obtain $b^{q}=|\mu(u)|^{p}$, and hence

$$
|\mu(u)|^{p} \leq \frac{c^{p} \varepsilon^{2 p}}{\delta^{p}}+\frac{2 \varepsilon^{2}}{\delta} \Delta|\mu(u)|^{p} .
$$

By Lemma 9.2, this implies that

$$
\int_{B_{R}(z)}|\mu(u)|^{p} \leq \frac{\pi(R+r)^{2} c^{p} \varepsilon^{2 p}}{\delta^{p}}+\frac{8 \varepsilon^{2}}{r^{2} \delta} \int_{B_{R+r}(z)}|\mu(u)|^{p}
$$


for all $z \in \mathbb{C}$ and $R, r>0$ such that $B_{R+r}(z) \subset \Omega$. Use the last inequality repeatedly, with $R$ replaced by $R+r, R+2 r, \ldots, R+(p-1) r$, to obtain the estimate

$$
\int_{B_{R}(z)}|\mu(u)|^{p} \leq c^{\prime} \varepsilon^{2 p}
$$

for every $z \in \mathbb{C}$ such that $B_{R+p r}(z) \subset \Omega$. Now choose $R$ and $r$ such that $B_{R+p r}(z) \subset \Omega$ for every $z \in K$. Cover $K$ by finitely many balls of radius $R$ to obtain the inequality

$$
\int_{K}|\mu(u)|^{p} \leq c^{\prime \prime} \varepsilon^{2 p}
$$

By equation (9.2), the function

$$
z \longmapsto|\mu(u(z))|^{p}+\frac{c^{p} \varepsilon^{2 p-2}}{8 \delta^{p-1}}\left|z-z_{0}\right|^{2}
$$

is subharmonic in $\Omega$ for every $z_{0} \in \mathbb{C}$. Hence, by the mean value inequality, we have

$$
|\mu(u(z))|^{p} \leq \frac{c^{p} \varepsilon^{2 p-2}}{8 \pi R^{2}} \int_{B_{R}}|z|^{2}+\frac{1}{\pi R^{2}} \int_{B_{R}(z)}|\mu(u)|^{p} \leq c^{\prime \prime \prime} \varepsilon^{2 p-2}
$$

for $z \in \mathbb{C}$ such that $B_{R+p r}(z) \subset \Omega$. This proves the lemma.

Lemma 9.2. Let $u: B_{R+r} \rightarrow \mathbb{R}$ be a $C^{2}$-function and $f, g: B_{R+r} \rightarrow \mathbb{R}$ be continuous such that

$$
f \leq g+\Delta u, \quad u \geq 0, \quad f \geq 0, g \geq 0 .
$$

Then

$$
\int_{B_{R}} f \leq \int_{B_{R+r}} g+\frac{4}{r^{2}} \int_{B_{R+r} \backslash B_{R}} u .
$$

Moreover, if $g=c u$ then

$$
\frac{\pi}{2} \sup _{B_{R}} u \leq\left(c+\frac{4}{r^{2}}\right) \int_{B_{R+r}} u .
$$

Proof. For $0 \leq s \leq r$, we have

$$
\int_{B_{R}} f-\int_{B_{R+r}} g \leq \int_{B_{R+s}}(f-g) \leq \int_{B_{R+s}} \Delta u=\int_{\partial B_{R+s}} \frac{\partial u}{\partial \nu},
$$

and hence

$$
\frac{\mathrm{d}}{\mathrm{d} s} \int_{\partial B_{R+s}} u=\int_{\partial B_{R+s}} \frac{\partial u}{\partial \nu}+\frac{1}{R+s} \int_{\partial B_{R+s}} u \geq \int_{B_{R}} f-\int_{B_{R+r}} g .
$$

Integrate this inequality over the interval $0 \leq s \leq t$ to obtain

$$
\int_{B_{R}} f-\int_{B_{R+r}} g \leq \frac{1}{t} \int_{\partial B_{R+t}} u \leq \frac{2}{r} \int_{\partial B_{R+t}} u
$$


for $r / 2 \leq t \leq r$. The first inequality follows by integrating this inequality over the interval $r / 2 \leq t \leq r$. The second inequality was proved in $[6$, Lemma 7.3].

Lemma 9.3. Let $C_{0}>0, \Omega \subset \mathbb{C}$ be a bounded open set, $K \subset \Omega$ be a compact subset, and $\lambda: \bar{\Omega} \rightarrow(0, \infty)$ be a smooth function. Then there are positive constants $\varepsilon_{0}$ and $c$ such that the following holds. If $0<\varepsilon \leq \varepsilon_{0}$ and $(u, \Phi, \Psi)$ is a solution of (9.1) satisfying

$$
\left\|v_{s}\right\|_{L^{\infty}(\Omega)}+\varepsilon^{-1}\|\mu(u)\|_{L^{\infty}(\Omega)} \leq C_{0},
$$

then

$$
\begin{gathered}
\varepsilon^{-1}\|\mu(u)\|_{L^{p}(K)}+\left\|L_{u}^{*} v_{s}\right\|_{L^{p}(K)}+\left\|L_{u}^{*} J v_{s}\right\|_{L^{p}(K)} \\
+\varepsilon\left\|\nabla_{A, s} v_{s}\right\|_{L^{p}(K)}+\varepsilon\left\|\nabla_{A, t} v_{s}\right\|_{L^{p}(K)} \\
\leq c \varepsilon^{2 / p}\left(\left\|v_{s}\right\|_{L^{2}(\Omega)}+\varepsilon^{-1}\|\mu(u)\|_{L^{2}(\Omega)}\right)
\end{gathered}
$$

for $2 \leq p \leq \infty$.

Proof. Consider the functions $u_{0}, v_{0}: \Omega \rightarrow \mathbb{R}$ given by

$$
\begin{gathered}
u_{0}:=\frac{1}{2}\left(\left|v_{s}\right|^{2}+\frac{\lambda^{2}}{\varepsilon^{2}}|\mu(u)|^{2}\right) \\
v_{0}:=\frac{1}{2}\left(\left|\nabla_{A, s} v_{s}\right|^{2}+\left|\nabla_{A, t} v_{s}\right|^{2}+\frac{\lambda^{4}}{\varepsilon^{4}}\left|L_{u} \mu(u)\right|^{2}+\frac{\lambda^{2}}{\varepsilon^{2}}\left|L_{u}^{*} v_{s}\right|^{2}+\frac{\lambda^{2}}{\varepsilon^{2}}\left|L_{u}^{*} J v_{s}\right|^{2}\right) .
\end{gathered}
$$

We prove that there exists a constant $c_{0}>0$ such that

$$
\Delta u_{0} \geq v_{0}-c_{0} u_{0}
$$

To see this, recall from the proof of Lemma 9.1 that

$$
\frac{1}{2} \Delta|\mu(u)|^{2}=\left|L_{u}^{*} v_{s}\right|^{2}+\left|L_{u}^{*} J v_{s}\right|^{2}+\frac{\lambda^{2}}{\varepsilon^{2}}\left|L_{u} \mu(u)\right|^{2}-2\left\langle\mu(u), \rho\left(v_{s}, v_{t}\right)\right\rangle,
$$

and hence

$$
\begin{aligned}
\frac{1}{2 \varepsilon^{2}} \Delta & \left(\lambda^{2}|\mu(u)|^{2}\right) \\
= & \frac{\Delta \lambda^{2}}{2 \varepsilon^{2}}|\mu(u)|^{2}+\frac{\lambda^{2}}{2 \varepsilon^{2}} \Delta|\mu(u)|^{2}+\frac{\partial_{s} \lambda^{2}}{\varepsilon^{2}} \partial_{s}|\mu(u)|^{2} \\
& +\frac{\partial_{t} \lambda^{2}}{\varepsilon^{2}} \partial_{t}|\mu(u)|^{2} \\
= & \frac{\Delta \lambda^{2}}{2 \varepsilon^{2}}|\mu(u)|^{2}+\frac{2 \partial_{t} \lambda^{2}}{\varepsilon^{2}}\left\langle\mu(u), L_{u}^{*} v_{s}\right\rangle-\frac{2 \partial_{s} \lambda^{2}}{\varepsilon^{2}}\left\langle\mu(u), L_{u}^{*} J v_{s}\right\rangle \\
& +\frac{\lambda^{2}}{\varepsilon^{2}}\left|L_{u}^{*} v_{s}\right|^{2}+\frac{\lambda^{2}}{\varepsilon^{2}}\left|L_{u}^{*} J v_{s}\right|^{2}+\frac{\lambda^{4}}{\varepsilon^{4}}\left|L_{u} \mu(u)\right|^{2} \\
& -\frac{2 \lambda^{2}}{\varepsilon^{2}}\left\langle\mu(u), \rho\left(v_{s}, v_{t}\right)\right\rangle .
\end{aligned}
$$


Moreover, by Lemma B.4 and Lemma C.3,

$$
\begin{aligned}
& \left(\nabla_{A, s} \nabla_{A, s}+\nabla_{A, t} \nabla_{A, t}\right) v_{s} \\
& =\left(\nabla_{A, t} \nabla_{A, s}-\nabla_{A, s} \nabla_{A, t}\right) v_{t}+\nabla_{A, s}\left(\nabla_{A, s} v_{s}+\nabla_{A, t} v_{t}\right)-\nabla_{A, t}\left(\nabla_{A, s} v_{t}-\nabla_{A, t} v_{s}\right) \\
& =-R\left(v_{s}, v_{t}\right) v_{t}-\nabla_{v_{t}} X_{\kappa}(u)-\nabla_{A, s}\left(J L_{u} \kappa\right)-\nabla_{A, t}\left(L_{u} \kappa\right) \\
& =-R\left(v_{s}, v_{t}\right) v_{t}+\frac{\lambda^{2}}{\varepsilon^{2}} J \nabla_{v_{s}} X_{\mu(u)}(u)+\frac{\lambda^{2}}{\varepsilon^{2}}\left(\nabla_{v_{s}} J\right) L_{u} \mu(u)+\frac{2 \lambda^{2}}{\varepsilon^{2}} \nabla_{v_{t}} X_{\mu(u)}(u) \\
& (9.5) \\
& \quad+\frac{\lambda^{2}}{\varepsilon^{2}} L_{u} L_{u}^{*} v_{s}-\frac{\lambda^{2}}{\varepsilon^{2}} J L_{u} L_{u}^{*} J v_{s}+\frac{\partial_{s} \lambda^{2}}{\varepsilon^{2}} J L_{u} \mu(u)+\frac{\partial_{t} \lambda^{2}}{\varepsilon^{2}} L_{u} \mu(u) .
\end{aligned}
$$

Hence

$$
\begin{aligned}
\frac{1}{2} \Delta\left|v_{s}\right|^{2}= & \left|\nabla_{A, s} v_{s}\right|^{2}+\left|\nabla_{A, t} v_{s}\right|^{2}+\left\langle v_{s},\left(\nabla_{A, s} \nabla_{A, s}+\nabla_{A, t} \nabla_{A, t}\right) v_{s}\right\rangle \\
= & \left|\nabla_{A, s} v_{s}\right|^{2}+\left|\nabla_{A, t} v_{s}\right|^{2}+\frac{\lambda^{2}}{\varepsilon^{2}}\left|L_{u}^{*} v_{s}\right|^{2}+\frac{\lambda^{2}}{\varepsilon^{2}}\left|L_{u}^{*} J v_{s}\right|^{2} \\
& -\frac{3 \lambda^{2}}{\varepsilon^{2}}\left\langle\mu(u), \rho\left(v_{s}, v_{t}\right)\right\rangle+\frac{\lambda^{2}}{\varepsilon^{2}}\left\langle v_{s},\left(\nabla_{v_{s}} J\right) L_{u} \mu(u)\right\rangle \\
& -\left\langle v_{s}, R\left(v_{s}, v_{t}\right) v_{t}\right\rangle-\frac{\partial_{s} \lambda^{2}}{\varepsilon^{2}}\left\langle L_{u}^{*} J v_{s}, \mu(u)\right\rangle+\frac{\partial_{t} \lambda^{2}}{\varepsilon^{2}}\left\langle L_{u}^{*} v_{s}, \mu(u)\right\rangle .
\end{aligned}
$$

Combining this with the formula for $\Delta\left(\lambda^{2}|\mu(u)|^{2}\right) / 2 \varepsilon^{2}$, we obtain

$$
\begin{aligned}
\Delta u_{0}= & \left|\nabla_{A, s} v_{s}\right|^{2}+\left|\nabla_{A, t} v_{s}\right|^{2}+\frac{2 \lambda^{2}}{\varepsilon^{2}}\left|L_{u}^{*} v_{s}\right|^{2}+\frac{2 \lambda^{2}}{\varepsilon^{2}}\left|L_{u}^{*} J v_{s}\right|^{2}+\frac{\lambda^{4}}{\varepsilon^{4}}\left|L_{u} \mu(u)\right|^{2} \\
& -\frac{5 \lambda^{2}}{\varepsilon^{2}}\left\langle\mu(u), \rho\left(v_{s}, v_{t}\right)\right\rangle+\frac{3 \partial_{t} \lambda^{2}}{\varepsilon^{2}}\left\langle\mu(u), L_{u}^{*} v_{s}\right\rangle-\frac{3 \partial_{s} \lambda^{2}}{\varepsilon^{2}}\left\langle\mu(u), L_{u}^{*} J v_{s}\right\rangle \\
& +\frac{\Delta \lambda^{2}}{2 \varepsilon^{2}}|\mu(u)|^{2}+\frac{\lambda^{2}}{\varepsilon^{2}}\left\langle v_{s},\left(\nabla_{v_{s}} J\right) L_{u} \mu(u)\right\rangle-\left\langle v_{s}, R\left(v_{s}, v_{t}\right) v_{t}\right\rangle .
\end{aligned}
$$

The first row on the right is bounded below by $2 v_{0}$. Moreover, by assumption, the image of $u$ is contained in the compact set $\left\{|\mu(x)| \leq \varepsilon C_{0}\right\}$. Hence the last six terms can be estimated from below by $v_{0}-c_{0} u_{0}$ for some constant $c_{0}$ whenever $\varepsilon$ is sufficiently small. Thus we have proved the inequality (9.4). Hence, by Lemma 9.2, there exist constants $\varepsilon_{0}>0$ and $c_{0}^{\prime}>0$ such that

$$
\sup _{K} u_{0}+\int_{K} v_{0} \leq c_{0}^{\prime} \int_{\Omega} u_{0}
$$

for every $\varepsilon \in\left(0, \varepsilon_{0}\right]$. Since $|\mu(u)| \leq C_{0} \varepsilon_{0}$ and zero is a regular value of $\mu$ there is an inequality $\left|L_{u} \eta\right| \geq \delta|\eta|$ whenever $\varepsilon_{0}$ is sufficiently small. Thus we have proved equation (9.3) for $p=2$ as well as

$$
\left\|v_{s}\right\|_{L^{\infty}(K)}+\varepsilon^{-1}\|\mu(u)\|_{L^{\infty}(K)} \leq c_{0}^{\prime}\left(\left\|v_{s}\right\|_{L^{2}(\Omega)}+\varepsilon^{-1}\|\mu(u)\|_{L^{2}(\Omega)}\right) .
$$


Now let us define $u_{1}: \Omega \rightarrow \mathbb{R}$ by

$$
u_{1}:=\frac{1}{2}\left|\nabla_{A, s} v_{s}\right|^{2} .
$$

We shall prove that there exist positive constants $\delta_{1}, c_{1}$, and $\varepsilon_{0}$ such that

$$
\Delta\left(u_{0}+\varepsilon^{2} u_{1}\right) \geq-c_{1} u_{0}
$$

for $0<\varepsilon \leq \varepsilon_{0}$. We consider the equation

$\Delta u_{1}=\left|\nabla_{A, s} \nabla_{A, s} v_{s}\right|^{2}+\left|\nabla_{A, t} \nabla_{A, s} v_{s}\right|^{2}+\left\langle\left(\nabla_{A, s} \nabla_{A, s}+\nabla_{A, t} \nabla_{A, t}\right) \nabla_{A, s} v_{s}, \nabla_{A, s} v_{s}\right\rangle$, and use the formula

$$
\left(\nabla_{A, s} \nabla_{A, s}+\nabla_{A, t} \nabla_{A, t}\right) \nabla_{A, s} v_{s}=\mathrm{I}+\mathrm{II}+\mathrm{III},
$$

where

$$
\begin{aligned}
& \mathrm{I}:=\left(\nabla_{A, t} \nabla_{A, s}-\nabla_{A, s} \nabla_{A, t}\right) \nabla_{A, t} v_{s} \\
&=-R\left(v_{s}, v_{t}\right) \nabla_{A, t} v_{s}+\frac{\lambda^{2}}{\varepsilon^{2}} \nabla_{\nabla_{A, t} v_{s}} X_{\mu}(u), \\
& \mathrm{II}:=\nabla_{A, t}\left(\nabla_{A, t} \nabla_{A, s}-\nabla_{A, s} \nabla_{A, t}\right) v_{s} \\
&=-\nabla_{A, t} R\left(v_{s}, v_{t}\right) v_{s}+\frac{\lambda^{2}}{\varepsilon^{2}} \nabla_{A, t}\left(\nabla_{v_{s}} X_{\mu}(u)\right)+\frac{\partial_{t} \lambda^{2}}{\varepsilon^{2}} \nabla_{v_{s}} X_{\mu}(u), \\
& \mathrm{III}:=\nabla_{A, s}\left(\nabla_{A, s} \nabla_{A, s}+\nabla_{A, t} \nabla_{A, t}\right) v_{s} \\
&=\nabla_{A, s}\left(-R\left(v_{s}, v_{t}\right) v_{t}+\frac{\lambda^{2}}{\varepsilon^{2}} L_{u} L_{u}^{*} v_{s}-\frac{\lambda^{2}}{\varepsilon^{2}} J L_{u} L_{u}^{*} J v_{s}+\frac{\lambda^{2}}{\varepsilon^{2}}\left(\nabla_{v_{s}} J\right) X_{\mu}(u)\right. \\
&\left.\quad \quad \frac{\lambda^{2}}{\varepsilon^{2}} J \nabla_{v_{s}} X_{\mu}(u)+\frac{2 \lambda^{2}}{\varepsilon^{2}} \nabla_{v_{t}} X_{\mu}(u)+\frac{\partial_{s} \lambda^{2}}{\varepsilon^{2}} J X_{\mu}(u)+\frac{\partial_{t} \lambda^{2}}{\varepsilon^{2}} X_{\mu}(u)\right) .
\end{aligned}
$$

Here we abbreviate $X_{\mu}(u)=X_{\mu(u)}(u)=L_{u} \mu(u)$. The last equality for III follows from equation (9.5). Now consider the tensors $\nabla^{2} J$ and $\nabla^{2} X_{\eta}$ defined by

$$
\begin{aligned}
\nabla^{2} J(X, Y, Z) & :=\nabla_{X}\left(\left(\nabla_{Y} J\right) Z\right)-\left(\nabla_{\nabla_{X} Y} J\right) Z-\left(\nabla_{Y} J\right) \nabla_{X} Z \\
\nabla^{2} X_{\eta}(Y, Z) & :=\nabla_{Y}\left(\nabla_{Z} X_{\eta}\right)-\nabla_{\nabla_{Y} Z} X_{\eta},
\end{aligned}
$$

for $\eta \in \mathfrak{g}$ and $X, Y, Z \in \operatorname{Vect}(M)$. Then

$$
\begin{aligned}
\nabla_{A, s}\left(\left(\nabla_{v_{s}} J\right) L_{u} \mu(u)\right)= & \nabla^{2} J\left(v_{s}, v_{s}, L_{u} \mu(u)\right)+\left(\nabla_{\nabla_{A, s} v_{s}} J\right) L_{u} \mu(u) \\
& +\left(\nabla_{v_{s}} J\right) \nabla_{v_{s}} X_{\mu(u)}(u)-\left(\nabla_{v_{s}} J\right) L_{u} L_{u}^{*} J v_{s}, \\
\nabla_{A, t}\left(\nabla_{v_{s}} X_{\mu(u)}(u)\right)= & \nabla^{2} X_{\mu(u)}\left(v_{t}, v_{s}\right)+\nabla_{\nabla_{A, t} v_{s}} X_{\mu(u)}(u) \nabla_{v_{s}} X_{L_{u}^{*} v_{s}}(u), \\
\nabla_{A, s}\left(R\left(v_{s}, v_{t}\right) v_{t}\right)= & \nabla R\left(v_{s}, v_{s}, v_{t}, v_{t}\right)+R\left(\nabla_{A, s} v_{s}, v_{t}\right) v_{t} \\
& \left.+R\left(v_{s}, \nabla_{A, s}\left(J v_{s}\right)\right) v_{t}\right)+R\left(v_{s}, v_{t}\right) \nabla_{A, s}\left(J v_{s}\right) .
\end{aligned}
$$


Hence, by a term-by-term inspection, we obtain an inequality

$$
\varepsilon^{2}\left\langle\nabla_{A, s} v_{s}, \mathrm{I}+\mathrm{II}+\mathrm{III}\right\rangle \geq-c u_{0}-v_{0}
$$

for $\varepsilon>0$ sufficiently small. Note, in particular, that the term $\varepsilon^{2}\left\langle\nabla_{A, s} v_{s}\right.$, III $\rangle$ contains the two positive summands $\lambda^{2}\left|L_{u}^{*} \nabla_{A, s} v_{s}\right|^{2}$ and $\lambda^{2}\left|L_{u}^{*} J \nabla_{A, s} v_{s}\right|^{2}$. Since $\Delta u_{0} \geq v_{0}-c_{0} u_{0}$ the last inequality implies equation (9.8) with $c_{1}:=c+c_{0}$. Now it follows from equations (9.7) and (9.8), Lemma 9.2, and the formula

$$
\nabla_{A, t} v_{s}=-J \nabla_{A, s} v_{s}-\left(\nabla_{v_{s}} J\right) v_{s}+\left(\frac{\lambda}{\varepsilon}\right)^{2} L_{u} \mu(u)
$$

that equation (9.3) holds for $p=\infty$. For $2<p<\infty$, the result follows by interpolation.

\section{Proof of Theorem 3.3}

Theorem 10.1. Assume $(\mathrm{H} 1)$ and $(\mathrm{H} 4)$, let $\bar{B} \in H_{2}(\bar{M} ; \mathbb{Z})$ be a nontorsion homology class, and denote $B:=\kappa(\bar{B}) \in H_{2}\left(M_{\mathrm{G}} ; \mathbb{Z}\right)$. Then, for every $C>0$, there exist positive constants $\varepsilon_{0}$ and $c_{0}$ such that for every $\varepsilon \in\left(0, \varepsilon_{0}\right]$ the following holds. If $(u, A) \in \widetilde{\mathcal{M}}_{B, \Sigma}^{\varepsilon}$ satisfies

$$
\left\|d_{A} u\right\|_{L^{\infty}} \leq C, \quad u(P) \subset M^{C},
$$

then $(u, A) \in \widetilde{\mathcal{T} \varepsilon}\left(\widetilde{\mathcal{M}}_{B, \Sigma}^{0}\left(c_{0}\right)\right)$.

Proof. Suppose the assertion is false. Then there exist a constant $C>0$ and sequences $\varepsilon_{i} \rightarrow 0$ and $\left(u_{i}, A_{i}\right) \in \widetilde{\mathcal{M}}_{B, \Sigma}^{\varepsilon_{i}}$ such that

$$
\left\|d_{A_{i}} u_{i}\right\|_{L^{\infty}} \leq C, \quad u_{i}(P) \subset M^{C}, \quad\left(u_{i}, A_{i}\right) \notin \widetilde{\mathcal{T}}^{\varepsilon_{i}}\left(\mathcal{M}_{B, \Sigma}^{0}(i)\right) .
$$

Here $\varepsilon_{i}$ is chosen smaller than the number $\varepsilon_{0}(i)$ required for the definition of the map $\mathcal{T}^{\varepsilon_{i}}$. We prove in four steps that there exist an integer $i_{0} \in \mathbb{N}$, positive constants $c$ and $c_{0}$, and sequences

$$
\left(u_{i 0}, A_{i 0}\right) \in \widetilde{\mathcal{M}}_{B, \Sigma}^{0}\left(c_{0}-1\right), \quad \zeta_{i 0}=\left(\xi_{i 0}, \alpha_{i 0}\right) \in T_{\left(u_{i 0}, A_{i 0}\right)} \mathcal{B},
$$

such that

$$
\left(u_{i}, A_{i}\right)=\left(\exp _{u_{i 0}}\left(\xi_{i 0}\right), A_{i 0}+\alpha_{i 0}\right), \quad\left\|\zeta_{i 0}\right\|_{1, p, \varepsilon_{i}} \leq c \varepsilon_{i}^{2 / p+1},
$$

for every $i \geq i_{0}$. For $i$ sufficiently large, it then follows from Theorem 8.1 that $\left(u_{i}, A_{i}\right) \in \widetilde{\mathcal{T}}^{\varepsilon_{i}}\left(\widetilde{\mathcal{M}}_{B, \Sigma}^{0}\left(c_{0}\right)\right)$, in contradiction to our assumption.

Step 1. There exist constants $c>0$ and $i_{0} \in \mathbb{N}$ such that, for $i \geq i_{0}$ and $2 \leq p \leq \infty$, we have,

$$
\varepsilon_{i}^{-1}\left\|\mu\left(u_{i}\right)\right\|_{L^{p}}+\left\|L_{u_{i}}^{*} d_{A_{i}} u_{i}\right\|_{L^{p}}+\left\|L_{u_{i}}^{*} J d_{A_{i}} u_{i}\right\|_{L^{p}}+\varepsilon_{i}\left\|\nabla_{A_{i}}{ }^{*} d_{A_{i}} u_{i}\right\|_{L^{p}} \leq c \varepsilon_{i}^{2 / p} .
$$


By the graph construction in Appendix A, it suffices to establish the estimate under the hypothesis that $J$ is independent of $z \in \Sigma$. Namely,

$$
\begin{aligned}
L_{\tilde{u}_{i}}^{*} d_{A_{i}} \tilde{u}_{i} & =L_{u_{i}}^{*} d_{A_{i}} u_{i}, \quad L_{\tilde{u}_{i}}^{*} \tilde{J} d_{A_{i}} \tilde{u}_{i}=L_{u_{i}}^{*} J d_{A_{i}} u_{i}, \\
\tilde{\nabla}_{A_{i}}{ }^{*} d_{A_{i}} \tilde{u}_{i} & =\left(\nabla_{\Sigma}{ }^{*} \mathrm{id}, \nabla_{A_{i}}{ }^{*} d_{A_{i}} u_{i}\right),
\end{aligned}
$$

where $\tilde{u}_{i}=\left(\pi, u_{i}\right): P \rightarrow \tilde{M}=\Sigma \times M, \tilde{\nabla}_{A}$ is the connection induced by $A$ on $\tilde{u}_{i}^{*} T \tilde{M} / \mathrm{G}$, and id $\in \Omega^{1}(\Sigma, T \Sigma)$. Hence we can use the results of Section 9. Since $\left\|d_{A_{i}} u_{i}\right\|_{L^{\infty}} \leq C$ and $u_{i}(\Omega) \subset M^{C}$, the pair $\left(\tilde{u}_{i}, A_{i}\right)$ satisfies the hypotheses of Lemma 9.1 and so the sequence $\varepsilon_{i}{ }^{-3 / 2} \tilde{\mu}\left(\tilde{u}_{i}\right)$ is uniformly bounded. Hence there exists a constant $c_{0}>0$ such that

$$
\left\|d_{A_{i}} u_{i}\right\|_{L^{\infty}}+\varepsilon_{i}^{-3 / 2}\left\|\mu\left(u_{i}\right)\right\|_{L^{\infty}} \leq c_{0}
$$

for every $i$. This implies that, in local holomorphic coordinates on $\Sigma$, the pair $\left(\tilde{u}_{i}, A_{i}\right)$ satisfies the hypotheses of Lemma 9.3 for $i$ sufficiently large. Hence the estimate holds in local holomorphic coordinates on $\Sigma$ with $u_{i}$ replaced by $\tilde{u}_{i}$. Hence, by a partition of unity argument, it holds globally.

Step 2. There exists an integer $i_{0} \in \mathbb{N}$ and a constant $c>0$ such that, for every $i \geq i_{0}$, there exists a unique $\eta_{i} \in \Omega^{0}\left(\Sigma, \mathfrak{g}_{P}\right)$ such that

$$
\mu\left(\exp _{u_{i}}\left(J L_{u_{i}} \eta_{i}\right)\right)=0, \quad\left\|\eta_{i}\right\|_{L^{\infty}} \leq c\left\|\mu\left(u_{i}\right)\right\|_{L^{\infty}} .
$$

Define $u_{i}^{\prime}: P \rightarrow M$ and $A_{i}^{\prime} \in \mathcal{A}(P)$ by

$$
u_{i}^{\prime}:=\exp _{u_{i}}\left(J L_{u_{i}} \eta_{i}\right), \quad L_{u_{i}^{\prime}}^{*} d_{A_{i}^{\prime}} u_{i}^{\prime}=0,
$$

so that $d_{A_{i}^{\prime}} u_{i}^{\prime} \in \Omega^{1}\left(\Sigma, H_{u_{i}^{\prime}}\right)$, and let $\zeta_{i}:=\left(J L_{u_{i}} \eta_{i}, A_{i}^{\prime}-A_{i}\right)$. Then there exists a constant $c^{\prime}>0$ such that, for $i \geq i_{0}$,

$$
\left\|\zeta_{i}\right\|_{1, p, \varepsilon_{i}} \leq c^{\prime} \varepsilon_{i}^{1+2 / p}, \quad\left\|\bar{\partial}_{J, A_{i}^{\prime}}\left(u_{i}^{\prime}\right)\right\|_{L^{p}} \leq c^{\prime} \varepsilon_{i}^{1+2 / p}, \quad\left\|d_{A_{i}^{\prime}} u_{i}^{\prime}\right\|_{L^{\infty}} \leq c^{\prime} .
$$

The existence of $\eta_{i}$ for large $i$ follows from the implicit function theorem for the map $\eta \mapsto \mu\left(\exp _{u_{i}(p)}\left(J L_{u_{i}(p)} \eta\right)\right)$. This sequence satisfies an estimate of the form

$$
\left\|\eta_{i}\right\|_{L^{p}} \leq c_{1}\left\|\mu\left(u_{i}\right)\right\|_{L^{p}} \leq c_{2} \varepsilon^{1+2 / p}
$$

for every $i \geq i_{0}$ and every $p \in[2, \infty]$. Here the constants $c_{1}$ and $c_{2}$ are independent of $i$ and $p$, and the second inequality follows from Step 1. For $p=\infty$ there is actually a better estimate (by $\varepsilon^{3 / 2}$ instead of $\varepsilon$ ), but we shall not use this here. In the following, we suppress the subscript $i$ and write $u, u^{\prime}, A, A^{\prime}, \varepsilon$ instead of $u_{i}, u_{i}^{\prime}, A_{i}, A_{i}^{\prime}, \varepsilon_{i}$, respectively. We establish the required estimates in local holomorphic coordinates on $\Sigma$. As in Remark 4.1, we write $A^{\prime}=\Phi^{\prime} d s+\Psi^{\prime} d t$ for some Lie-algebra-valued functiona $\Phi^{\prime}$ and $\Psi^{\prime}$, and denote

$$
v_{s}^{\prime}:=\partial_{s} u^{\prime}+X_{\Phi^{\prime}}\left(u^{\prime}\right), \quad v_{t}^{\prime}:=\partial_{t} u^{\prime}+X_{\Psi^{\prime}}\left(u^{\prime}\right) .
$$


Then $L_{u^{\prime}}^{*} v_{s}^{\prime}=L_{u^{\prime}}^{*} v_{t}^{\prime}=0$. We assume that the functions $u, u^{\prime}, \Phi, \Psi, \Phi^{\prime}$, and $\Psi^{\prime}$ are defined on an open set $\Omega \subset \mathbb{C}$ and fix any compact subset $K \subset \Omega$. We must prove the estimates

$$
\begin{gathered}
\|(\xi, \varphi, \psi)\|_{1, p, \varepsilon} \leq c \varepsilon^{1+2 / p}, \quad\left\|v_{s}^{\prime}+J v_{t}^{\prime}\right\|_{L^{p}} \leq c \varepsilon^{1+2 / p}, \\
\left\|v_{s}^{\prime}\right\|_{L^{\infty}}+\left\|v_{t}^{\prime}\right\|_{L^{\infty}} \leq c
\end{gathered}
$$

on the subset $K$, where

$$
\xi:=J L_{u} \eta, \quad \varphi:=\Phi^{\prime}-\Phi, \quad \psi:=\Psi^{\prime}-\Psi .
$$

Abbreviate $E_{i}:=E_{i}\left(u, J L_{u} \eta\right), i=1,2$. Then

$$
\nabla_{A, t} \xi=J L_{u} \nabla_{A, t} \eta+\left(\nabla_{v_{t}} J+\partial_{t} J\right) L_{u} \eta+J \nabla_{v_{t}} X_{\eta}(u) .
$$

Hence, by Lemma C.3,

$$
v_{t}^{\prime}-E_{1} v_{t}=L_{u^{\prime}} \psi+E_{2} \nabla_{A, t} \xi=L_{u^{\prime}} \psi+E_{2} J L_{u} \nabla_{A, t} \eta+R_{t} \eta
$$

where

$$
R_{t} \eta:=E_{2}\left(\nabla_{v_{t}} J+\partial_{t} J\right) L_{u} \eta+E_{2} J \nabla_{v_{t}} X_{\eta}(u)
$$

Hence

$$
d \mu\left(u^{\prime}\right)\left(v_{t}^{\prime}-E_{1} v_{t}\right)=d \mu\left(u^{\prime}\right) E_{2} J L_{u} \nabla_{A, t} \eta+d \mu\left(u^{\prime}\right) R_{t} \eta .
$$

Since $L_{u}^{*}=d \mu(u) J$, we have

$$
\begin{aligned}
L_{u}^{*} L_{u} \nabla_{A, t} \eta= & \left(d \mu(u)-d \mu\left(u^{\prime}\right) E_{2}\right) J L_{u} \nabla_{A, t} \eta \\
& +d \mu\left(u^{\prime}\right)\left(v_{t}^{\prime}-E_{1} v_{t}\right)-d \mu\left(u^{\prime}\right) R_{t} \eta,
\end{aligned}
$$

and, since $d \mu\left(u^{\prime}\right) v_{t}^{\prime}=0$,

$$
\begin{aligned}
L_{u}^{*} L_{u} \nabla_{A, t} \eta= & \left(d \mu(u)-d \mu\left(u^{\prime}\right) E_{2}\right) J L_{u} \nabla_{A, t} \eta \\
& +\left(d \mu(u)-d \mu\left(u^{\prime}\right) E_{1}\right) v_{t}+L_{u}^{*} J v_{t}-d \mu\left(u^{\prime}\right) R_{t} \eta .
\end{aligned}
$$

It follows that

$$
\begin{aligned}
\left\|\nabla_{A, t} \eta\right\|_{L^{p}} & \leq c_{2}\left(\|\eta\|_{L^{\infty}}\left\|\nabla_{A, t} \eta\right\|_{L^{p}}+\left\|L_{u}^{*} J v_{t}\right\|_{L^{p}}+\|\eta\|_{L^{p}}\right) \\
& \leq c_{3}\left(\varepsilon^{1+2 / p}\left\|\nabla_{A, t} \eta\right\|_{L^{p}}+\varepsilon^{2 / p}\right) .
\end{aligned}
$$

If $\varepsilon$ is sufficiently small this gives

$$
\left\|\nabla_{A, t} \eta\right\|_{L^{p}} \leq c_{4} \varepsilon^{2 / p}, \quad\left\|\nabla_{A, t} \eta\right\|_{L^{\infty}} \leq c_{4} .
$$

Here the second inequality follows from a similar argument as the first. Combining these inequalities with (10.3) we obtain

$$
\left\|\nabla_{A, t} \xi\right\|_{L^{p}} \leq c_{5} \varepsilon^{2 / p}, \quad\left\|\nabla_{A, t} \xi\right\|_{L^{\infty}} \leq c_{5} .
$$

In order to estimate $\psi$ we apply the operator $L_{u^{\prime}}^{*}$ to equation (10.4) and use the formula $L_{u^{\prime}}^{*} v_{t}^{\prime}=0$ to obtain

$$
L_{u^{\prime}}^{*} L_{u^{\prime}} \psi=\left(L_{u}^{*}-L_{u^{\prime}}^{*} E_{1}\right) v_{t}-L_{u}^{*} v_{t}-L_{u^{\prime}}^{*} E_{2} J L_{u} \nabla_{A, t} \eta-L_{u^{\prime}}^{*} R_{t} \eta .
$$


Combining this with Step 1 and the estimate for $\nabla_{A, t} \eta$, we obtain

$$
\|\psi\|_{L^{p}} \leq c_{6} \varepsilon^{2 / p}, \quad\|\psi\|_{L^{\infty}} \leq c_{6} .
$$

Hence, by equation (10.4),

$$
\left\|v_{t}^{\prime}-E_{1} v_{t}\right\|_{L^{p}} \leq c_{7} \varepsilon^{2 / p}, \quad\left\|v_{t}^{\prime}-E_{1} v_{t}\right\|_{L^{\infty}} \leq c_{7} .
$$

Similarly,

$$
\begin{gathered}
\left\|\nabla_{A, s} \eta\right\|_{L^{p}}+\|\varphi\|_{L^{p}}+\left\|v_{s}^{\prime}-E_{1} v_{s}\right\|_{L^{p}} \leq c_{7} \varepsilon^{2 / p} \\
\left\|\nabla_{A, s} \eta\right\|_{L^{\infty}}+\|\varphi\|_{L^{\infty}}+\left\|v_{s}^{\prime}-E_{1} v_{s}\right\|_{L^{\infty}} \leq c_{7} .
\end{gathered}
$$

Now use equation (10.4) again to obtain

$$
\begin{aligned}
v_{s}^{\prime}+J\left(u^{\prime}\right) v_{t}^{\prime}= & L_{u^{\prime}}\left(\varphi-\nabla_{A, t} \eta\right)+J L_{u^{\prime}}\left(\psi+\nabla_{A, s} \eta\right) \\
& +\left(E_{2} J L_{u}-J L_{u^{\prime}}\right) \nabla_{A, s} \eta+J\left(E_{2} J L_{u}-J L_{u^{\prime}}\right) \nabla_{A, t} \eta \\
& +E_{1} v_{s}+J E_{1} v_{t}+\left(R_{s}+J R_{t}\right) \eta \\
= & \pi_{u^{\prime}}\left(\left(E_{2} J L_{u}-J L_{u^{\prime}}\right) \nabla_{A, s} \eta+J\left(E_{2} J L_{u}-J L_{u^{\prime}}\right) \nabla_{A, t} \eta\right) \\
& +\pi_{u^{\prime}}\left(\left(J E_{1}-E_{1} J\right) v_{t}+\left(R_{s}+J R_{t}\right) \eta\right) .
\end{aligned}
$$

The second equality uses the fact that $v_{s}+J v_{t}=0$ and that the 1-form $\bar{\partial}_{J, A^{\prime}}\left(u^{\prime}\right)$ takes values in $H_{u^{\prime}}$. It follows that

$$
\left\|v_{s}^{\prime}+J\left(u^{\prime}\right) v_{t}^{\prime}\right\|_{L^{p}} \leq c_{8} \varepsilon^{1+2 / p} .
$$

It remains to show that

$$
\left\|\nabla_{A, s} \varphi\right\|_{L^{p}}+\left\|\nabla_{A, t} \varphi\right\|_{L^{p}}+\left\|\nabla_{A, s} \psi\right\|_{L^{p}}+\left\|\nabla_{A, t} \psi\right\|_{L^{p}} \leq c \varepsilon^{2 / p-1} .
$$

To estimate the term $\nabla_{A, t} \psi$, differentiate equation (10.4) with respect to $t$. Then apply the operator $d \mu\left(u^{\prime}\right)$ to the resulting expression to eliminate $\nabla_{A, t} \psi$ and obtain an estimate of the form

$$
\left\|\nabla_{A, t} \nabla_{A, t} \eta\right\|_{L^{p}} \leq c_{9} \varepsilon^{2 / p-1}
$$

Then apply the operator $L_{u^{\prime}}^{*}$ to the equation obtained from differentiating equation (10.4), and estimate $\nabla_{A, t} \psi$ using the upper bound found for $\nabla_{A, t} \nabla_{A, t} \eta$. The estimate for $\nabla_{A, s} \psi$ is obtained in a similar manner. To estimate $\nabla_{A, s} \varphi$ and $\nabla_{A, t} \varphi$, we begin with the identity

$$
v_{s}^{\prime}-E_{1} v_{s}=L_{u^{\prime}} \varphi+E_{2} J L_{u} \nabla_{A, s} \eta+R_{s} \eta
$$

instead of equation (10.4) and then follow the same procedure.

Step 3. There exist an integer $i_{0} \in \mathbb{N}$, a constant $c>0$, and a sequence $\left(u_{i}^{\prime \prime}, A_{i}^{\prime \prime}\right) \in \widetilde{\mathcal{M}}_{B, \Sigma}^{0}$ such that, for $i \geq i_{0}$, we have

$$
\begin{gathered}
u_{i}^{\prime \prime}=\exp _{u_{i}^{\prime}}\left(\xi_{i}^{\prime}\right), \quad \xi_{i}^{\prime} \in \Omega^{0}\left(\Sigma, H_{u_{i}^{\prime}}\right), \\
\left\|\xi_{i}^{\prime}\right\|_{W^{1, p}}+\left\|A_{i}^{\prime \prime}-A_{i}^{\prime}\right\|_{L^{p}} \leq c \varepsilon_{i}^{1+2 / p}, \quad\left\|d_{A_{i}^{\prime \prime}} u_{i}^{\prime \prime}\right\|_{L^{\infty}} \leq c .
\end{gathered}
$$


By Step 2,

$$
\sup _{i}\left\|d_{A_{i}^{\prime}} u_{i}^{\prime}\right\|_{L^{\infty}}<\infty \text {. }
$$

Hence the induced maps $\bar{u}_{i}^{\prime}: \Sigma \rightarrow \bar{M}$ form a sequence of approximate $\bar{J}$-holomorphic curves which satisfy a uniform $L^{\infty}$-bound on their first derivatives. Hence, by (H4) and Theorem 2.3, there is nearby a true $\bar{J}_{-}$ holomorphic curve $\bar{u}_{i}^{\prime \prime}: \Sigma \rightarrow \bar{M}$ whose $W^{1, p}$-distance to $\bar{u}_{i}^{\prime}$ is controlled by the $L^{p}$-norm of $\bar{\partial}_{\bar{J}}\left(\bar{u}_{i}^{\prime}\right)$. Now this $\bar{J}$-holomorphic curve has a unique lift $u_{i}^{\prime \prime}: P \rightarrow \mu^{-1}(0)$ of the form

$$
u_{i}^{\prime \prime}=\exp _{u_{i}^{\prime}}\left(\xi_{i}^{\prime}\right), \quad \xi_{i}^{\prime} \in \Omega^{0}\left(\Sigma, H_{u_{i}^{\prime}}\right) .
$$

Let $A_{i}^{\prime \prime} \in \mathcal{A}(P)$ be the connection determined by $u_{i}^{\prime \prime}$ via $L_{u_{i}^{\prime \prime}}^{*} d_{A_{i}^{\prime \prime}} u_{i}^{\prime \prime}=0$. Then

$$
\left\|\xi_{i}^{\prime}\right\|_{W^{1, p}} \leq c_{1}\left\|\bar{\partial}_{J, A_{i}^{\prime}}\left(u_{i}^{\prime}\right)\right\|_{L^{p}} \leq c_{2} \varepsilon_{i}^{1+2 / p}
$$

Here the last inequality follows from Step 2. Since $L_{u_{i}^{\prime}}^{*} d_{A_{i}^{\prime}} u_{i}^{\prime}=L_{u_{i}^{\prime \prime}}^{*} d_{A_{i}^{\prime \prime}} u_{i}^{\prime \prime}=$ 0 , we obtain

$$
\left\|A_{i}^{\prime \prime}-A_{i}^{\prime}\right\|_{L^{p}} \leq c_{3} \varepsilon_{i}^{1+2 / p}
$$

In particular, these inequalities together give a uniform $W^{1, p}$-bound on the $\bar{J}$-holomorphic curves $\bar{u}_{i}^{\prime \prime}: \Sigma \rightarrow \bar{M}$. Hence, by the elliptic bootstrapping techniques for $J$-holomorphic curves, the sequence $\bar{u}_{i}^{\prime \prime}$ satisfies a uniform $L^{\infty}$ bound on the first derivatives. This proves Step 3 .

Unfortunately, the estimate on $A_{i}^{\prime \prime}-A_{i}^{\prime}$ in Step 3 is only in the $L^{p}$-norm and not in the $W^{1, p}$-norm. A further modification of the pair $\left(u_{i}^{\prime \prime}, A_{i}^{\prime \prime}\right)$ is required to improve this estimate.

Step 4. There exist an integer $i_{0} \in \mathbb{N}$, a constant $c>0$, and a sequence of gauge transformations $g_{i} \in \mathcal{G}(P)$ such that the sequence

$$
\left(u_{i 0}, A_{i 0}\right):=g_{i}^{*}\left(u_{i}^{\prime \prime}, A_{i}^{\prime \prime}\right) \in \widetilde{\mathcal{M}}_{B, \Sigma}^{0}
$$

satisfies the following. For $i \geq i_{0}$ the original sequence $\left(u_{i}, A_{i}\right)$ has the form

$$
\left(u_{i}, A_{i}\right)=\left(\exp _{u_{i 0}}\left(\xi_{i}\right), A_{i 0}+\alpha_{i}\right)
$$

where $\zeta_{i}:=\left(\xi_{i}, \alpha_{i}\right) \in T_{\left(u_{i 0}, A_{i 0}\right)} \mathcal{B}$ satisfies (10.1).

The idea is to choose $g_{i}$ for large $i$ such that

$$
u_{i}=\exp _{u_{i 0}}\left(\xi_{i}\right), \quad L_{u_{i 0}}^{*} \xi_{i}=0, \quad u_{i 0}:=g_{i}^{-1} u_{i}^{\prime \prime} .
$$

This can be done by using pointwise, for every $p \in P$, the implicit function theorem to obtain the local slice condition. This suffices to obtain the missing estimates for the first derivatives of $g_{i}^{*} A_{i}^{\prime \prime}-A_{i}$.

By equation (10.2) and Step 2, the distance between $u_{i}$ and $u_{i}^{\prime}$ is unformly bounded by a constant times $\varepsilon_{i}^{3 / 2}$, while the distance in the $W^{1, p}$-norm is bounded by a constant times $\varepsilon_{i}^{2 / p}$. By Step 3 , the distance between $u_{i}^{\prime}$ and 
$u_{i}^{\prime \prime}$ is bounded in the $W^{1, p}$-norm by a constant times $\varepsilon_{i}^{1+2 / p}$. Hence there exists a sequence of smooth sections $\xi_{i} \in \Omega^{0}\left(\Sigma, u_{i}^{*} T M / \mathrm{G}\right)$ and a constant $c>0$ such that

$$
u_{i}^{\prime \prime}=\exp _{u_{i}}\left(\xi_{i}\right), \quad\left\|\xi_{i}\right\|_{L^{\infty}} \leq c_{1} \varepsilon_{i}, \quad\left\|\nabla_{A_{i}} \xi_{i}\right\|_{L^{p}} \leq c_{1} \varepsilon_{i}^{2 / p} .
$$

Moreover, the sequence $d_{A_{i}^{\prime \prime}} u_{i}^{\prime \prime}$ is uniformly bounded in the $L^{\infty}$-norm and

$$
\left\|A_{i}^{\prime \prime}-A_{i}\right\|_{L^{p}} \leq c_{1} \varepsilon^{2 / p}, \quad\left\|d_{A_{i}^{\prime \prime}} u_{i}^{\prime \prime}-E_{1}\left(u_{i}, \xi_{i}\right) d_{A_{i}} u_{i}\right\|_{L^{p}} \leq c_{1} \varepsilon^{2 / p} .
$$

Here the last inequality follows from the identity

$$
\begin{aligned}
d_{A_{i}^{\prime \prime}} u_{i}^{\prime \prime}= & E_{1}\left(u_{i}, \xi_{i}\right) d_{A_{i}} u_{i}+E_{2}\left(u_{i}, \xi_{i}\right) \nabla_{A_{i}} \xi_{i} \\
& +E_{1}\left(u_{i}, \xi_{i}\right) X_{A_{i}^{\prime \prime}-A_{i}}\left(u_{i}\right)+E_{2}\left(u_{i}, \xi_{i}\right) \nabla_{\xi_{i}} X_{A_{i}^{\prime \prime}-A_{i}}\left(u_{i}\right),
\end{aligned}
$$

which in turn follows from Lemma C.1. Now, by the inverse function theorem for the map

$$
\mathrm{G} \times \operatorname{ker} L_{x}^{*} \rightarrow M:(g, \xi) \mapsto g^{-1} \exp _{x}(\xi),
$$

there exists a constant $c_{2}>0$ and (unique) sequences $g_{i} \in \mathcal{G}(P)$ and $\xi_{i}^{\prime \prime} \in$ $\Omega^{0}\left(\Sigma, u_{i}^{\prime \prime *} T M / \mathrm{G}\right)$ such that

$$
u_{i}=g_{i}^{-1} \exp _{u_{i}^{\prime \prime}}\left(\xi_{i}^{\prime \prime}\right), \quad L_{u_{i}^{\prime \prime}}^{*} \xi_{i}^{\prime \prime}=0, \quad\left\|\xi_{i}^{\prime \prime}\right\|_{L^{\infty}} \leq c_{2} \varepsilon_{i}, \quad\left\|g_{i}-\mathbb{1}\right\|_{L^{\infty}} \leq c_{2} \varepsilon_{i} .
$$

Define

$$
u_{i 0}:=g_{i}^{-1} u_{i}^{\prime \prime}, \quad A_{i 0}:=g_{i}{ }^{*} A_{i}^{\prime \prime}, \quad \xi_{i 0}:=g_{i}^{-1} \xi_{i}^{\prime \prime}, \quad \alpha_{i 0}:=A_{i}-A_{i 0} .
$$

We shall prove that the pair $\left(\xi_{i 0}, \alpha_{i 0}\right)$ satisfies equation (10.1). To see this note first that

$$
u_{i}=\exp _{u_{i 0}}\left(\xi_{i 0}\right), \quad A_{i}=A_{i 0}+\alpha_{i 0} .
$$

The endomorphism $E_{1}\left(u_{i}, \xi_{i}\right) g_{i}^{-1} E_{1}\left(u_{i}^{\prime \prime}, \xi_{i}^{\prime \prime}\right)$ of $u_{i}^{\prime \prime *} T M$ is $\varepsilon_{i}$-close to the identity, $d_{A_{i 0}} u_{i 0}=g_{i}^{-1} d_{A_{i}^{\prime \prime}} u_{i}^{\prime \prime}$, and

$$
\begin{aligned}
& E_{1}\left(u_{i}, \xi_{i}\right)\left(d_{A_{i}} u_{i}-E_{1}\left(u_{i 0}, \xi_{i 0}\right) d_{A_{i 0}} u_{i 0}\right) \\
& \quad=E_{1}\left(u_{i}, \xi_{i}\right) d_{A_{i}} u_{i}-d_{A_{i}^{\prime \prime}} u_{i}^{\prime \prime}+\left(1-E_{1}\left(u_{i}, \xi_{i}\right) g^{-1} E_{1}\left(u_{i}^{\prime \prime}, \xi_{i}^{\prime \prime}\right)\right) d_{A_{i}^{\prime \prime}} u_{i}^{\prime \prime} .
\end{aligned}
$$

Hence, by equations (10.6), there is an estimate

$$
\left\|d_{A_{i}} u_{i}-E_{1}\left(u_{i 0}, \xi_{i 0}\right) d_{A_{i 0}} u_{i 0}\right\|_{L^{p}} \leq c_{3} \varepsilon_{i}^{2 / p}, \quad\left\|d_{A_{i 0}} u_{i 0}\right\|_{L^{\infty}} \leq c_{3}
$$

for all $i$. Hence, by Corollary C.4, there exists a constant $c_{4}>0$ such that

$$
\left\|\alpha_{i 0}\right\|_{L^{p}} \leq c_{4} \varepsilon_{i}^{2 / p}, \quad\left\|\alpha_{i 0}\right\|_{L^{\infty}} \leq c_{4} .
$$

Next observe that, by Lemma C.1,

$$
\begin{aligned}
d_{A_{i}} u_{i}= & E_{1}\left(u_{i 0}, \xi_{i 0}\right) d_{A_{i 0}} u_{i 0}+E_{2}\left(u_{i 0}, \xi_{i 0}\right) \nabla_{A_{i 0}} \xi_{i 0} \\
& +E_{1}\left(u_{i 0}, \xi_{i 0}\right) X_{\alpha_{i 0}}\left(u_{i 0}\right)+E_{2}\left(u_{i 0}, \xi_{i 0}\right) \nabla_{\xi_{i 0}} X_{\alpha_{i 0}}\left(u_{i 0}\right) .
\end{aligned}
$$


Hence there exists a constant $c_{5}>0$ such that

$$
\left\|\nabla_{A_{i 0}} \xi_{i 0}\right\|_{L^{p}} \leq c_{5} \varepsilon_{i}^{2 / p}, \quad\left\|\nabla_{A_{i 0}} \xi_{i 0}\right\|_{L^{\infty}} \leq c_{5},
$$

for all $i$. Thus we have proved that

$$
\begin{aligned}
\left\|\xi_{i 0}\right\|_{L^{p}}+\varepsilon_{i}\left\|\alpha_{i 0}\right\|_{L^{p}}+\varepsilon_{i}\left\|\nabla_{A_{i 0}} \xi_{i 0}\right\|_{L^{p}} & \leq c_{6} \varepsilon_{i}^{1+2 / p}, \\
\left\|\xi_{i 0}\right\|_{L^{\infty}}+\varepsilon_{i}\left\|\alpha_{i 0}\right\|_{L^{\infty}}+\varepsilon_{i}\left\|\nabla_{A_{i 0}} \xi_{i 0}\right\|_{L^{\infty}} & \leq c_{6} \varepsilon_{i} .
\end{aligned}
$$

It remains to estimate the $L^{p}$-norm of the first derivatives of $\alpha_{i 0}$. We drop the subscript $i$ and write $u, u_{0}, A, A_{0}, \xi_{0}, \alpha_{0}$ instead of $u_{i}, u_{i 0}, A_{i}, A_{i 0}, \xi_{i 0}, \alpha_{i 0}$. Moreover, we use local coordinates on $\Sigma$ as in Step 2 and write

$$
\begin{array}{ll}
A_{0}:=\Phi_{0} d s+\Psi_{0} d t, & A:=\Phi d s+\Psi d t, \\
v_{0 s}:=\partial_{s} u_{0}+L_{u_{0}} \Phi_{0}, & v_{s}:=\partial_{s} u+L_{u} \Phi, \\
v_{0 t}:=\partial_{t} u_{0}+L_{u_{0}} \Psi_{0}, & v_{t}:=\partial_{t} u+L_{u} \Psi,
\end{array}
$$

and $\varphi_{0}:=\Phi-\Phi_{0}$ and $\psi_{0}:=\Psi-\Psi_{0}$. Consider the formula

$$
\nabla_{A, s} v_{s}+J \nabla_{A, t} v_{s}=-\left(\nabla_{v_{t}} J\right) v_{s}+\frac{\lambda^{2}}{\varepsilon^{2}} J L_{u} \mu(u) .
$$

By Step 1, we have

$$
\left\|\nabla_{A, s} v_{s}+J \nabla_{A, t} v_{s}\right\|_{L^{p}} \leq c_{7} \varepsilon^{2 / p-1},
$$

and hence, by elliptic regularity for the Cauchy-Riemann operator,

$$
\left\|\nabla_{A, s} v_{s}\right\|_{L^{p}}+\left\|\nabla_{A, t} v_{s}\right\|_{L^{p}} \leq c_{8} \varepsilon^{2 / p-1} .
$$

Moreover, since $L_{u_{0}}^{*} \xi_{0}=0$, it follows from Lemma C.2 that

$$
\begin{aligned}
L_{u_{0}}^{*} \nabla_{A_{0}, t} \nabla_{A_{0}, s} \xi_{0} & =\nabla_{A_{0}, t}\left(L_{u_{0}}^{*} \nabla_{A_{0}, s} \xi_{0}\right)-\rho\left(v_{0 t}, \nabla_{A_{0}, s} \xi_{0}\right) \\
& =-\nabla_{A_{0}, t} \rho\left(v_{0 s}, \xi_{0}\right)-\rho\left(v_{0 t}, \nabla_{A_{0}, s} \xi_{0}\right),
\end{aligned}
$$

and hence

$$
\left\|L_{u_{0}}^{*} \nabla_{A_{0}, t} \nabla_{A_{0}, s} \xi_{0}\right\| \leq c_{10} \varepsilon^{2 / p} .
$$

Here we use the fact that, by elliptic bootstrapping for $\bar{J}$-holomorphic curves, there is a uniform $L^{p}$-bound on $\nabla_{A_{0}, t} v_{0 s}$. Now consider the inequality

$$
\begin{aligned}
\left|\nabla_{A, t} \varphi_{0}\right| & \leq c_{11}\left|L_{u}^{*} L_{u} \nabla_{A, t} \varphi_{0}\right| \\
& \leq c_{11}\left|\left(L_{u}^{*}-L_{u_{0}}^{*} E_{2}^{-1}\right) L_{u} \nabla_{A, t} \varphi_{0}\right|+c_{11}\left|L_{u_{0}}^{*} E_{2}^{-1} L_{u} \nabla_{A, t} \varphi_{0}\right|
\end{aligned}
$$

Since the operator $\left(L_{u}^{*}-L_{u_{0}}^{*} E_{2}^{-1}\right) L_{u}$ is small, we obtain

$$
\left\|\nabla_{A, t} \varphi_{0}\right\|_{L^{p}} \leq c_{12}\left\|L_{u_{0}}^{*} E_{2}^{-1} L_{u} \nabla_{A, t} \varphi_{0}\right\|_{L^{p}} \text {. }
$$

Now use Lemma C.5 and the estimates (10.7), (10.8), and (10.9) to obtain

$$
\left\|\nabla_{A, t} \varphi_{0}\right\|_{L^{p}} \leq c_{13} \varepsilon^{2 / p-1} .
$$

The terms $\left\|\nabla_{A, s} \varphi_{0}\right\|_{L^{p}},\left\|\nabla_{A, t} \psi_{0}\right\|_{L^{p}}$, and $\left\|\nabla_{A, s} \varphi_{0}\right\|_{L^{p}}$ are estimated similarly. This proves Step 4. 
It follows from Step 4 and Theorem 8.1 that $\left(u_{i}, A_{i}\right) \in \widetilde{\mathcal{T}}_{i}^{\varepsilon}\left(\widetilde{\mathcal{M}}_{B, \Sigma}^{0}\left(c_{0}\right)\right)$ for some constant $c_{0}$ and $i$ sufficiently large. This contradicts our assumption and hence proves the theorem.

\section{Vortices}

In this section, we examine the finite energy solutions of equation (1.1) over the complex plane $\Sigma=\mathbb{C}$. The equations have the form

$$
\begin{aligned}
\partial_{s} u+X_{\Phi}(u)+J\left(\partial_{t} u+X_{\Psi}(u)\right) & =0, \\
\partial_{s} \Psi-\partial_{t} \Phi+[\Phi, \Psi]+\mu(u) & =0,
\end{aligned}
$$

where $u: \mathbb{C} \rightarrow M$ and $\Phi, \Psi: \mathbb{C} \rightarrow \mathfrak{g}$. The energy of the triple $(u, \Phi, \Psi)$ is given by

$$
E(u, \Phi, \Psi):=\int_{\mathbb{C}}\left(\left|\partial_{s} u+X_{\Phi}(u)\right|^{2}+|\mu(u)|^{2}\right) \mathrm{d} s \mathrm{~d} t
$$

The vortex equations (11.1) and the energy are invariant under the action of the gauge group $\mathcal{G}:=C^{\infty}(\mathbb{C}, \mathrm{G})$ by

$$
g^{*}(u, \Phi, \Psi):=\left(g^{-1} u, g^{-1} \partial_{s} g+g^{-1} \Phi g, g^{-1} \partial_{t} g+g^{-1} \Psi g\right) .
$$

A solution of equation (11.1) is said to be in radial gauge if

$$
\cos \theta \Phi\left(r \mathrm{e}^{\mathrm{i} \theta}\right)+\sin \theta \Psi\left(r \mathrm{e}^{\mathrm{i} \theta}\right)=0
$$

for every $\theta \in \mathbb{R}$ and every sufficiently large $r \geq 0$. It is said to be bounded if $\sup _{\mathbb{C}}|\mu(u)|<\infty$.

Proposition 11.1. Assume (H1) and (H2). Suppose that $(u, \Phi, \Psi)$ is a smooth bounded finite energy solution of equation (11.1) in radial gauge. Then there exists a $W^{1,2}$-function $x: \mathbb{R} / 2 \pi \mathbb{Z} \rightarrow M$ and an $L^{2}$-function $\eta: \mathbb{R} / 2 \pi \mathbb{Z} \rightarrow \mathfrak{g}$ such that

$$
\dot{x}+X_{\eta}(x)=0, \quad \mu(x)=0,
$$

$$
\lim _{r \rightarrow \infty} \sup _{\theta \in \mathbb{R}} d\left(u\left(r \mathrm{e}^{\mathrm{i} \theta}\right), x(\theta)\right)=0, \quad \lim _{r \rightarrow \infty} \int_{0}^{2 \pi}\left|\eta(\theta)-\eta_{r}(\theta)\right|^{2} \mathrm{~d} \theta=0,
$$

where $\eta_{r}(\theta):=r \cos (\theta) \Psi\left(r \mathrm{e}^{\mathrm{i} \theta}\right)-r \sin \theta \Phi\left(r \mathrm{e}^{\mathrm{i} \theta}\right)$. Moreover,

$$
E(u, \Phi, \Psi)=\int_{\mathbb{C}} u^{*} \omega,
$$

there is a constant $\delta>0$ such that

$$
\lim _{r \rightarrow \infty} \sup _{\theta \in \mathbb{R}} r^{2+\delta}\left(\left|\partial_{s} u+X_{\Phi}(u)\right|^{2}+|\mu(u)|^{2}\right)=0,
$$

where $s+i t=: r \mathrm{e}^{\mathrm{i} \theta}$, and $\sup _{\mathbb{C}}(f \circ u) \leq c$, where $c$ is as in hypothesis $(H 2)$ and $\mu^{-1}(0) \subset f^{-1}([0, c])$. If $(\mathrm{H} 3)$ holds then $E(u, \Phi, \Psi)$ is an integer multiple of $\hbar=\tau N$. 
Note that the removable singularity theorem for $J$-holomorphic curves is a corollary of Proposition 11.1 (consider the special case $\mathrm{G}=\{\mathbb{1}\}$ and $M=\bar{M}$ ). Before entering into the proof, we introduce the notion of the local equivariant symplectic action. The definition of this local action functional relies on the following lemma. We identify $S^{1} \cong \mathbb{R} / 2 \pi \mathbb{Z}$.

Lemma 11.2. Assume (H1). Then there are positive constants $\delta$ and $c$ such that the following holds. If $x: S^{1} \rightarrow M$ and $\eta: S^{1} \rightarrow \mathfrak{g}$ are smooth loops such that $\sup _{S^{1}}|\mu(x)|<\delta$, then there is a point $x_{0} \in \mu^{-1}(0)$ and a smooth loop $g_{0}: S^{1} \rightarrow \mathrm{G}$ such that

$$
\begin{gathered}
c^{-1} \sup _{S^{1}}\left|\eta+\dot{g}_{0} g_{0}{ }^{-1}\right| \leq \ell(x, \eta):=\int_{0}^{2 \pi}\left|\dot{x}+X_{\eta}(x)\right| \mathrm{d} \theta, \\
d\left(x(\theta), g_{0}(\theta) x_{0}\right) \leq c(|\mu(x(\theta))|+\ell(x, \eta)) .
\end{gathered}
$$

Proof. Fix an almost complex structure $J \in \mathcal{J}_{\mathrm{G}}(M, \omega)$ and choose $r>0$ so small that the map $f: \mu^{-1}(0) \times\left\{\eta_{0} \in \mathfrak{g}|| \eta_{0} \mid<r\right\} \rightarrow M$, defined by

$$
f\left(x_{0}, \eta_{0}\right):=\exp _{x_{0}}\left(J L_{x_{0}} \eta_{0}\right),
$$

is an embedding. Choose $\delta>0$ so small that the set $\{|\mu|<\delta\}$ is contained in the image of $f$. Since $J L_{x_{0}}=d \mu\left(x_{0}\right)^{*}$, there is a constant $c_{0}>0$ such that

$$
\left|\eta_{0}\right| \leq c_{0}\left|\mu\left(f\left(x_{0}, \eta_{0}\right)\right)\right|
$$

for $x_{0} \in \mu^{-1}(0)$ and $\eta_{0} \in \mathfrak{g}$ with $\left|\eta_{0}\right| \leq r$. Let $c_{1}>0$ be an upper bound for the differential of $f^{-1}$ and denote

$$
c_{2}:=\sup _{\mu^{-1}(0)}\left|L_{x}\right| .
$$

Given a loop $x$ such that $|\mu(x)| \leq \delta$ define $\left(x_{0}(\theta), \eta_{0}(\theta)\right):=f^{-1}(x(\theta))$. Then

$$
d\left(x(\theta), x_{0}(0)\right) \leq d\left(x(\theta), x_{0}(\theta)\right)+\ell\left(x_{0}\right) \leq c_{0} c_{2}|\mu(x(\theta))|+c_{1} \ell(x),
$$

where $\ell(x):=\ell(x, 0)$. This proves the lemma in the case $\eta \equiv 0$.

Now consider the general case. Suppose $\delta$ has been chosen so small that $\mathrm{G}$ acts freely on the set $\{|\mu|<\delta\}$. Then there is a constant $c_{3}>0$ such that

$$
d(g, \mathbb{1}) \leq c_{3} d(g x, x)
$$

for $g \in \mathrm{G}$ and $x \in M$ with $|\mu(x)|<\delta$. Suppose $(x, \eta): S^{1} \rightarrow M \times \mathfrak{g}$ satisfies the assumptions of the lemma. Define $g: \mathbb{R} \rightarrow \mathrm{G}$ by

$$
\dot{g}+\eta g=0, \quad g(0)=\mathbb{1} .
$$

Then the length of the path $[0,2 \pi] \rightarrow M: \theta \mapsto g(\theta)^{-1} x(\theta)$ is equal to $\ell(x, \eta)$. Hence

$$
d(g(2 \pi), \mathbb{1}) \leq c_{3} d\left(g(2 \pi)^{-1} x(2 \pi), x(0)\right) \leq c_{3} \ell(x, \eta) .
$$


Choose $\eta_{1} \in \mathfrak{g}$ such that $g(2 \pi)=\exp \left(2 \pi \eta_{1}\right)$ and $\left|\eta_{1}\right| \leq c_{3} \ell(x, \eta) / 2 \pi$. Since $g(\theta+2 \pi)=g(\theta) g(2 \pi)$, the formula

$$
g_{0}(\theta):=g(\theta) \exp \left(-\theta \eta_{1}\right)
$$

defines a loop in G. It satisfies $\left|\eta+\dot{g}_{0} g_{0}^{-1}\right|=\left|\eta_{1}\right| \leq c_{3} \ell(x, \eta) / 2 \pi$. Hence the length of the loop

$$
y(\theta):=g_{0}(\theta)^{-1} x(\theta)
$$

is bounded by a constant times $\ell(x, \eta)$. By the first part of the proof, there is an $x_{0} \in \mu^{-1}(0)$ such that $d\left(y(\theta), x_{0}\right) \leq c(|\mu(y(\theta))|+\ell(y))$. Since $d\left(y(\theta), x_{0}\right)=d\left(x(\theta), g_{0}(\theta) x_{0}\right)$ and $|\mu(y(\theta))|=|\mu(x(\theta))|$, this proves the lemma.

Fix an almost complex structure $J \in \mathcal{J}_{\mathrm{G}}(M, \omega)$, let $\delta$ and $c$ be as in Lemma 11.2, and suppose that $2 c \delta$ is smaller than the injectivity radius of $M$ (with respect to the metric determined by $J$ ). Let $(x, \eta): S^{1} \rightarrow M \times \mathfrak{g}$ be a loop such that $\sup _{S^{1}}|\mu(x)|<\delta$ and $\ell(x, \eta)<\delta$. Then the local equivariant symplectic action of the pair $(x, \eta)$ is defined by

$$
\mathcal{A}(x, \eta):=-\int u^{*} \omega+\int_{0}^{2 \pi}\langle\mu(x(\theta)), \eta(\theta)\rangle \mathrm{d} \theta,
$$

where $x_{0} \in \mu^{-1}(0)$ and $g_{0}: S^{1} \rightarrow \mathrm{G}$ are as in Lemma 11.2, $\xi_{0}(\theta) \in T_{g_{0}(\theta) x_{0}} M$ is the unique small tangent vector such that

$$
x(\theta)=\exp _{g_{0}(\theta) x_{0}}\left(\xi_{0}(\theta)\right),
$$

and $u:[0,1] \times S^{1} \rightarrow M$ is defined by

$$
u(\tau, \theta):=\exp _{g_{0}(\theta) x_{0}}\left(\tau \xi_{0}(\theta)\right) .
$$

The local action is independent of the choice of $x_{0}$ and $g_{0}$.

Lemma 11.3. Assume (H1). There exist positive constants $\delta$ and $c$ such that the following holds. If $(x, \eta): S^{1} \rightarrow M$ is a smooth loop such that $\sup _{S^{1}}|\mu(x)|<\delta$ and $\ell(x, \eta)<\delta$, then

$$
|\mathcal{A}(x, \eta)| \leq c \int_{0}^{2 \pi}\left(\left|\dot{x}+X_{\eta}(x)\right|^{2}+|\mu(x)|^{2}\right) \mathrm{d} \theta .
$$

Proof. Let $\xi_{0} \in C^{\infty}\left(S^{1}, x_{0}^{*} T M\right)$ and $u:[0,1] \times S^{1} \rightarrow M$ be as above. Then the local equivariant symplectic action can be expressed in the form

$$
\mathcal{A}(x, \eta)=\int_{0}^{1} \int_{0}^{2 \pi} \omega\left(\partial_{\tau} u, \partial_{\theta} u+X_{\eta}(u)\right) \mathrm{d} \theta \mathrm{d} \tau .
$$

By Lemma 11.2, we have the pointwise inequality

$$
\left|\partial_{\tau} u\right|=\left|\xi_{0}\right|=d\left(x, g_{0} x_{0}\right) \leq c_{1}(|\mu(x)|+\ell(x, \eta)) .
$$

Moreover, by Lemma C.1,

$$
\partial_{\theta} u+X_{\eta}(u)=E_{1} L_{g_{0} x_{0}}\left(\eta+\dot{g}_{0} g_{0}^{-1}\right)+\tau E_{2}\left(\nabla \xi_{0}+\nabla_{\xi_{0}} X_{\eta}\left(g_{0} x_{0}\right)\right),
$$


where $E_{i}:=E_{i}\left(g_{0} x_{0}, \tau \xi_{0}\right)$ for $i=1,2$. With $\tau=1$ we obtain, by Lemma 11.2,

$$
\left|\nabla \xi_{0}+\nabla_{\xi_{0}} X_{\eta}\left(g_{0} x_{0}\right)\right| \leq c_{2}\left(\left|\dot{x}+X_{\eta}(x)\right|+\ell(x, \eta)\right) .
$$

This implies $\left|\partial_{\theta} u+X_{\eta}(u)\right| \leq c_{3}\left(\left|\dot{x}+X_{\eta}(x)\right|+\ell(x, \eta)\right)$ and hence

$$
\begin{aligned}
|\mathcal{A}(x, \eta)| & \leq c_{1} c_{3} \int_{0}^{2 \pi}(|\mu(x)|+\ell(x, \eta))\left(\left|\dot{x}+X_{\eta}(x)\right|+\ell(x, \eta)\right) \mathrm{d} \theta \\
& \leq c_{4} \int_{0}^{2 \pi}\left(\left|\dot{x}+X_{\eta}(x)\right|^{2}+|\mu(x)|^{2}\right) \mathrm{d} \theta .
\end{aligned}
$$

This proves the lemma.

Proof of Proposition 11.1. Let $(f, J)$ be as in $(\mathrm{H} 2)$ and let $(u, \Phi, \Psi)$ be a finite energy solution of equation (11.1) in radial gauge. We prove in seven steps that $(u, \Phi, \Psi)$ has the properties asserted in the proposition.

Step 1. $\lim _{r \rightarrow \infty} r^{2}\left(\left|\partial_{s} u+X_{\Phi}(u)\right|^{2}+|\mu(u)|^{2}\right)=0$ uniformly in $\theta$.

Abbreviate $v_{s}:=\partial_{s} u+L_{u} \Phi$ and $v_{t}:=\partial_{t} u+L_{u} \Psi$ as in Section 9. Let

$$
e:=\frac{1}{2}\left(\left|v_{s}\right|^{2}+|\mu(u)|^{2}\right) \text {. }
$$

Then the formula (9.6) with $\lambda=\varepsilon=1$ has the form

$$
\begin{aligned}
\Delta e= & \left|\nabla_{A, s} v_{s}\right|^{2}+\left|\nabla_{A, t} v_{s}\right|^{2}+2\left|L_{u}^{*} v_{s}\right|^{2}+2\left|L_{u}^{*} J v_{s}\right|^{2}+\left|L_{u} \mu(u)\right|^{2} \\
& -5\left\langle\mu(u), \rho\left(v_{s}, v_{t}\right)\right\rangle+\left\langle v_{s},\left(\nabla_{v_{s}} J\right) L_{u} \mu(u)\right\rangle-\left\langle v_{s}, R\left(v_{s}, v_{t}\right) v_{t}\right\rangle .
\end{aligned}
$$

Since $u(\mathbb{C})$ is contained in a compact subset of $M$ this gives an inequality

$$
\Delta e \geq-c_{1} e^{2}
$$

Namely, choose $\delta>0$ such that $L_{x}$ is injective whenever $|\mu(x)|^{2}<\delta$. Then the first term in the second row can be estimated from below by $-\left|L_{u} \mu(u)\right|^{2} / 2-c\left|v_{s}\right|^{4}$ whenever $e \leq \delta$. In case $e \geq \delta$ we can use the inequalities $\Delta e \geq-c\left(e+e^{2}\right)$ and $e \leq e^{2} / \delta$. Now it follows from [11, Lemma 4.3.2] that there is a constant $c_{2}>0$ such that

$$
|z|-r \geq c_{2} \quad \Longrightarrow \quad e(z) \leq \frac{8}{\pi r^{2}} \int_{B_{r}(z)} e .
$$

With $r:=|z| / 2$ this implies $\lim _{|z| \rightarrow \infty}|z|^{2} e(z)=0$.

Step 2. For $R>0$ sufficiently large, we have

$$
E\left(u, \Phi, \Psi ; \mathbb{C} \backslash B_{R}\right)=\mathcal{A}\left(x_{R}, \eta_{R}\right),
$$

where $x_{R}(\theta):=u\left(R \mathrm{e}^{i \theta}\right)$ and $\eta_{R}(\theta):=R \cos \theta \Psi\left(R e^{i \theta}\right)-R \sin \theta \Phi\left(R e^{i \theta}\right)$. 
The energy identity on $B_{R}=\{|z| \leq R\}$ has the form

$$
E\left(u, \Phi, \Psi ; B_{R}\right)=\int_{B_{R}} u^{*} \omega-\int_{0}^{2 \pi}\left\langle\mu\left(x_{R}(\theta)\right), \eta_{R}(\theta)\right\rangle \mathrm{d} \theta .
$$

For $R$ sufficiently large denote by $u_{R}:[0,1] \times S^{1} \rightarrow M$ the function used in the definition of the local symplectic action of $\left(x_{R}, \eta_{R}\right)$. Then $u_{R}(1, \theta)=$ $x_{R}(\theta)$ and $u_{R}(0, \theta)=g_{R}(\theta) x_{R 0}$ for some point $x_{R 0} \in \mu^{-1}(0)$ and some loop $g_{R}: S^{1} \rightarrow \mathrm{G}$. The homotopy class of the connected sum $v_{R}:=\left.u\right|_{B_{R}} \#\left(-u_{R}\right)$ (the orientation of $u_{R}$ is reversed) is independent of $R$. Hence the number

$$
\int v_{R}{ }^{*} \omega=E\left(u, \Phi, \Psi ; B_{R}\right)+\mathcal{A}\left(x_{R}, \eta_{R}\right)
$$

is independent of $R$. Since $\mathcal{A}\left(x_{R}, \eta_{R}\right)$ tends to zero as $R \rightarrow \infty$ it follows that

$$
E\left(u, \Phi, \Psi ; B_{R}\right)+\mathcal{A}\left(x_{R}, \eta_{R}\right)=E(u, \Phi, \Psi)
$$

for every sufficiently large number $R$. This proves Step 2 .

Step 3. $\sup _{\mathbb{C}}(f \circ u) \leq c$.

Suppose, by contradiction, that $\sup _{\mathbb{C}}(f \circ u)>c$. Then there exists a regular value $a$ of $f \circ u$ such that

$$
c<a<\sup _{\mathbb{C}}(f \circ u)
$$

Hence the set

$$
U:=\{z \in \mathbb{C} \mid f(u(z)) \geq a\}
$$

is a smooth submanifold of $\mathbb{C}$ with boundary. Since $\mu^{-1}(0) \subset f^{-1}([0, c])$ it follows from Step 1 that there exists a number $R>0$ such that

$$
\sup _{\mathbb{C} \backslash B_{R}}(f \circ u)<a<\sup _{\mathbb{C}}(f \circ u) .
$$

Hence $U$ is compact and has a nonempty boundary. By $(\mathrm{H} 2), \Delta(f \circ u) \geq 0$ in $U$ (see [2]). Hence

$$
0 \leq \int_{U} \Delta(f \circ u)=\int_{\partial U} \frac{\partial(f \circ u)}{\partial \nu}<0 .
$$

This contradiction proves Step 3.

Step 4. Consider equations (11.1) in polar coordinates $s+\mathrm{i} t=\mathrm{e}^{\tau+\mathrm{i} \theta}$. Define $\tilde{u}: \mathbb{R} \times S^{1} \rightarrow M$ and $\tilde{\Phi}, \tilde{\Psi}: \mathbb{R} \times S^{1} \rightarrow \mathfrak{g}$ by

$$
\begin{aligned}
\tilde{u}(\tau, \theta) & :=u\left(\mathrm{e}^{\tau+\mathrm{i} \theta}\right), \\
\tilde{\Phi}(\tau, \theta) & :=\mathrm{e}^{\tau} \cos \theta \Phi\left(\mathrm{e}^{\tau+\mathrm{i} \theta}\right)+\mathrm{e}^{\tau} \sin \theta \Psi\left(\mathrm{e}^{\tau+\mathrm{i} \theta}\right), \\
\tilde{\Psi}(\tau, \theta) & :=\mathrm{e}^{\tau} \cos \theta \Psi\left(\mathrm{e}^{\tau+\mathrm{i} \theta}\right)-\mathrm{e}^{\tau} \sin \theta \Phi\left(\mathrm{e}^{\tau+\mathrm{i} \theta}\right) .
\end{aligned}
$$


Then $d s \wedge d t=e^{2 \tau} d \tau \wedge d \theta, \Phi d s+\Psi d t=\tilde{\Phi} d \tau+\tilde{\Psi} d \theta$, and equations (11.1) are equivalent to

$$
\tilde{v}_{\tau}+J \tilde{v}_{\theta}=0, \quad \tilde{\kappa}+e^{2 \tau} \mu(\tilde{u})=0,
$$

where

$$
\tilde{v}_{\tau}:=\partial_{\tau} \tilde{u}+L_{\tilde{u}} \tilde{\Phi}, \quad \tilde{v}_{\theta}:=\partial_{\theta} \tilde{u}+L_{\tilde{u}} \tilde{\Psi}, \quad \tilde{\kappa}:=\partial_{\tau} \tilde{\Psi}-\partial_{\theta} \tilde{\Phi}+[\tilde{\Phi}, \tilde{\Psi}] .
$$

The radial gauge condition has the form $\tilde{\Phi}(\tau, \theta)=0$ for large $\tau$. The energy of the triple $(\tilde{u}, \tilde{\Phi}, \tilde{\Psi})$ is given by

$$
E(\tilde{u}, \tilde{\Phi}, \tilde{\Psi})=\int_{-\infty}^{\infty} \int_{0}^{2 \pi}\left(\left|\tilde{v}_{\tau}\right|^{2}+e^{2 \tau}|\mu(\tilde{u})|^{2}\right) \mathrm{d} \theta \mathrm{d} \tau .
$$

Step 5. There exist positive constants $c$ and $\delta$ such that, for every $\tau_{0} \geq 0$,

$$
\varepsilon\left(\tau_{0}\right):=\int_{\tau_{0}}^{\infty} \int_{0}^{2 \pi}\left(\left|\tilde{v}_{\tau}\right|^{2}+e^{2 \tau}|\mu(\tilde{u})|^{2}\right) d \theta d \tau \leq c e^{-\delta \tau_{0}} .
$$

By Step 2 and Lemma 11.3, we have

$$
\begin{aligned}
\varepsilon(\tau) & =\mathcal{A}\left(x_{e^{\tau}}, \eta_{e^{\tau}}\right) \\
& \leq \delta^{-1} \int_{0}^{2 \pi}\left(\left|\tilde{v}_{\tau}(\tau, \theta)\right|^{2}+e^{2 \tau}|\mu(\tilde{u}(\tau, \theta))|^{2}\right) \mathrm{d} \theta \\
& =-\delta^{-1} \varepsilon^{\prime}(\tau)
\end{aligned}
$$

for some constant $\delta>0$ and every sufficiently large real number $\tau$. In other words, the function $\tau \mapsto e^{\delta \tau} \varepsilon(\tau)$ is nonincreasing for $\tau$ sufficiently large. Hence there exists a real number $\tau_{0}$ such that

$$
\tau \geq \tau_{0} \Longrightarrow \varepsilon(\tau) \leq e^{-\delta\left(\tau-\tau_{0}\right)} \varepsilon\left(\tau_{0}\right) .
$$

Step 6. There exist positive constants $c$ and $\delta$ such that, for every $\tau_{0} \geq 0$,

$$
\begin{array}{r}
\sup _{\theta \in \mathbb{R}}\left(\left|\tilde{v}_{\tau}\left(\tau_{0}, \theta\right)\right|^{2}+e^{2 \tau_{0}}\left|\mu\left(\tilde{u}\left(\tau_{0}, \theta\right)\right)\right|^{2}\right) \leq c e^{-\delta \tau_{0}}, \\
\int_{\tau_{0}}^{\infty} \int_{0}^{2 \pi} e^{4 \tau} \mid \mu\left(\left.\tilde{u}(\tau, \theta)\right|^{2} \mathrm{~d} \theta \mathrm{d} \tau \leq c e^{-\delta \tau_{0}} .\right.
\end{array}
$$

By Step 1, the function $\left|\tilde{v}_{\tau}\right|^{2}+e^{2 \tau}|\mu(\tilde{u})|^{2}$ is bounded. Hence we can apply Lemma 9.3 to the open set $\Omega:=(-1,2) \times(-2 \pi, 4 \pi)$, the compact subset $K:=[0,1] \times[0,2 \pi]$, the function $\lambda(\tau, \theta):=e^{\tau}$, the constant $\varepsilon:=e^{-\tau_{0}}$, and the shifted functions $(\tau, \theta) \mapsto\left(\tilde{u}\left(\tau+\tau_{0}, \theta\right), \tilde{\Phi}\left(\tau+\tau_{0}, \theta\right), \tilde{\Psi}\left(\tau+\tau_{0}, \theta\right)\right)$. It follows from Lemma 9.3 with $p=2$ that there is a constant $c>0$ such that

$$
e^{4 \tau_{0}} \int_{\tau_{0}}^{\tau_{0}+1} \int_{0}^{2 \pi}|\mu(\tilde{u})|^{2} \mathrm{~d} \theta \mathrm{d} \tau \leq c \int_{\tau_{0}-1}^{\tau_{0}+2} \int_{-2 \pi}^{4 \pi}\left(\left|\tilde{v}_{\tau}\right|^{2}+e^{2 \tau_{0}}|\mu(\tilde{u})|^{2}\right) \mathrm{d} \theta \mathrm{d} \tau
$$


for every $\tau_{0} \in \mathbb{R}$. This implies

$$
\int_{\tau_{0}}^{\tau_{0}+1} \int_{0}^{2 \pi} e^{4 \tau}|\mu(\tilde{u})|^{2} \mathrm{~d} \theta \mathrm{d} \tau \leq 3 c e^{6} \int_{\tau_{0}-1}^{\tau_{0}+2} \int_{0}^{2 \pi}\left(\left|\tilde{v}_{\tau}\right|^{2}+e^{2 \tau}|\mu(\tilde{u})|^{2}\right) \mathrm{d} \theta \mathrm{d} \tau .
$$

Replace $\tau_{0}$ by $\tau_{0}+k$ and take the sum over all integers $k \geq 0$ to obtain

$$
\int_{\tau_{0}}^{\infty} \int_{0}^{2 \pi} e^{4 \tau}|\mu(\tilde{u})|^{2} \mathrm{~d} \theta \mathrm{d} \tau \leq 9 c e^{6} \int_{\tau_{0}-1}^{\infty} \int_{0}^{2 \pi}\left(\left|\tilde{v}_{\tau}\right|^{2}+e^{2 \tau}|\mu(\tilde{u})|^{2}\right) \mathrm{d} \theta \mathrm{d} \tau .
$$

Hence the $L^{2}$-estimate follows from Step 5 . To prove the $L^{\infty}$-estimate, use Lemma 9.3 again with $p=\infty$.

Step 7. There exists a $W^{1,2}$-function $x: S^{1} \rightarrow \mu^{-1}(0)$ and an $L^{2}$-function $\eta: S^{1} \rightarrow \mathfrak{g}$ such that

$$
\lim _{\tau \rightarrow \infty} \sup _{\theta \in \mathbb{R}} d(\tilde{u}(\tau, \theta), x(\theta))=0, \quad \lim _{\tau \rightarrow \infty} \int_{0}^{2 \pi}|\tilde{\Psi}(\tau, \theta)-\eta(\theta)|^{2} \mathrm{~d} \theta=0,
$$

and $\dot{x}+X_{\eta}(x)=0$. Moreover, $E(u, \Phi, \Psi)=\int u^{*} \omega$, and if (H3) holds then $E(u, \Phi, \Psi) \in \mathbb{Z} \hbar$.

By Hölder's inequality and the radial gauge assumption, we have, for $\tau_{1}>\tau_{0}$,

$$
\begin{aligned}
\left|\tilde{\Psi}\left(\tau_{1}, \theta\right)-\tilde{\Psi}\left(\tau_{0}, \theta\right)\right|^{2} & \leq\left(\int_{\tau_{0}}^{\tau_{1}} e^{2 \tau}|\mu(\tilde{u}(\tau, \theta))| \mathrm{d} \tau\right)^{2} \\
& \leq\left(\tau_{1}-\tau_{0}\right) \int_{\tau_{0}}^{\tau_{1}} e^{4 \tau}|\mu(\tilde{u}(\tau, \theta))|^{2} \mathrm{~d} \tau .
\end{aligned}
$$

Hence the existence of the $L^{2}$-limit of $\tilde{\Psi}$ follows from Step 6. That $\tilde{u}(\tau, \theta)$ converges uniformly as $\tau$ tends to infinity follows from the exponential decay of $\tilde{v}_{\tau}=\partial_{\tau} \tilde{u}$ in Step 6. That the limit is a $W^{1,2}$-function and satisfies $\dot{x}+X_{\eta}(x)=0$ follows from the fact that $\tilde{v}_{\theta}=\partial_{\theta} u+X_{\tilde{\Psi}}(\tilde{u})$ converges (exponentially) to zero as $\tau$ tends to infinity. That $E(u, \Phi, \Psi)=\int u^{*} \omega$ follows from the energy identity in the proof of Step 2 and the $L^{2}$-convergence of $\tilde{\Psi}$. That $E(u, \Phi, \Psi)$ is an integer multiple of $\hbar$ (when (H3) holds) follows from the proof of Step 2.

Remark 11.4. Every map $u: \mathbb{C} \rightarrow M$ that satisfies equations (11.2) and (11.3) determines an equivariant homology class $B=[u] \in H_{2}\left(M_{\mathrm{G}} ; \mathbb{Z}\right)$ as follows. Homotop $u$ to a map $v: D \rightarrow M$ such that

$$
v\left(e^{i \theta}\right)=g\left(e^{i \theta}\right) x_{0} .
$$

Now define a principal bundle $P \rightarrow S^{2} \cong\left(\mathbb{Z}_{2} \times D\right) / \sim$ by

$$
P:=\left(\mathbb{Z}_{2} \times D \times \mathrm{G}\right) / \sim, \quad\left(0, e^{i \theta}, h\right) \sim\left(1, e^{i \theta}, g\left(e^{i \theta}\right) h\right) .
$$

Then $v$ determines a G-equivariant map $w: P \rightarrow M$ by $w(0, z, h):=h^{-1} x_{0}$ and $w(1, z, h):=h^{-1} v(z)$. The equivariant homology class of $u$ is defined to be the equivariant homology class of $w$. 


\section{Proof of Theorem 1.1}

We begin by constructing a $\mathcal{G}$-equivariant smooth function from a suitable open subset of $\mathcal{B} \times P=C_{\mathrm{G}}^{\infty}(P, M) \times \mathcal{A}(P) \times P$ into a suitable finitedimensional approximation of EG. For positive constants $\delta$ and $r$ denote

$$
\begin{gathered}
M^{\delta}:=\{x \in M|| \mu(x) \mid \leq \delta\} \\
\mathcal{B}^{\delta, r}:=\left\{(u, A) \in C_{\mathrm{G}}^{\infty}(P, M) \times \mathcal{A}(P)\left|\exists z \in \Sigma \sup _{B_{r}(z)}\right| \mu \circ u \mid<\delta\right\} .
\end{gathered}
$$

Throughout we assume that $\mathrm{G}$ is a Lie subgroup of $\mathrm{U}(k)$. Then, for every integer $n \geq k$, a finite-dimensional approximation of the classifying space of $\mathrm{G}$ is the quotient

$$
\mathrm{BG}^{n}:=\mathrm{EG}^{n} / \mathrm{G}, \quad \mathrm{EG}^{n}:=\mathcal{F}(k, n):=\left\{\Theta \in \mathbb{C}^{n \times k} \mid \Theta^{*} \Theta=\mathbb{1}\right\} .
$$

There is an obvious embedding of the space $\mathrm{EG}^{n}=\mathcal{F}(k, n)$ (of unitary $k$ frames in $\left.\mathbb{C}^{n}\right)$ into $\mathcal{F}(k, n+1)$ for every $n \geq k$ and the direct limit is a model for EG. The homotopy groups of the approximations $\mathrm{EG}^{n}$ stabilize. Let $\delta>0$ be so small that $\mathrm{G}$ acts freely on $M^{\delta}$ and choose a smooth G-equivariant classifying map $\theta: M^{\delta} \rightarrow \mathrm{EG}^{m}$ for some integer $m \geq k$. Such a map exists for abstract homotopy-theoretic reasons but can also be constructed explicitly from local trivializations of the principal G-bundle $M^{\delta} \rightarrow M^{\delta} / \mathrm{G}$.

Proposition 12.1. Let $\theta: M^{\delta} \rightarrow \mathrm{EG}^{m}$ be as above. Then, for every $r>0$, there exist an integer $n \geq m$ and a map $\Theta: \mathcal{B}^{\delta, r} \times P \rightarrow \mathrm{EG}^{n}$ with the following properties.

(i) For $g \in \mathcal{G}, h \in \mathrm{G}$, and $(u, A, p) \in \mathcal{B}^{\delta, r} \times P$,

$$
\Theta\left(g^{-1} u, g^{*} A, p g(p)^{-1}\right)=\Theta(u, A, p)=h \Theta(u, A, p h) .
$$

(ii) $\Theta$ is smooth with respect to the $C^{0}$ Banach manifold structure on (the completion of) $\mathcal{B}^{\delta, r} \times P$.

(iii) Let $\iota: \mathrm{EG}^{m} \rightarrow \mathrm{EG}^{n}$ be the obvious inclusion. Then

$$
|\mu(u(p))|<\delta \quad \Longrightarrow \quad \Theta(u, A, p)=\iota \circ \theta(u(p)) .
$$

Proof. Cover $\Sigma$ by finitely many distinct balls $B_{r}\left(z_{i}\right), i=1, \ldots, \ell$, and choose points $p_{1}, \ldots, p_{\ell} \in P$ such that $\pi\left(p_{i}\right)=z_{i}$. Then, for every $(u, A) \in$ $\mathcal{B}^{\delta, r}$, there exists an $i \in\{1, \ldots, \ell\}$ such that $u\left(p_{i}\right) \in M^{\delta}$. Thus the open set $\mathcal{B}^{\delta, r} \times P$ is contained in the finite union of the following open sets $\mathcal{U}_{i j}$, $i, j=1, \ldots, \ell$. Choose $\varepsilon>0$ so small that $\theta$ extends to an equivariant function (still denoted by $\theta$ ) from $M^{\delta+\varepsilon}$ to $\mathrm{EG}^{m}$ and define

$$
\begin{aligned}
\mathcal{U}_{0} & :=\{(u, A, p)|| \mu(u(p)) \mid<\delta+\varepsilon\}, \\
\mathcal{U}_{i j} & :=\left\{(u, A, p)\left|\pi(p) \in B_{r}\left(z_{i}\right),\right| \mu\left(u\left(p_{j}\right)\right) \mid<\delta+\varepsilon\right\} .
\end{aligned}
$$


For every smooth path $\gamma:[0,1] \rightarrow \Sigma$ and any two points $p_{0} \in \pi^{-1}(\gamma(0))$ and $p_{1} \in \pi^{-1}(\gamma(1))$ the holonomy $\rho_{A}\left(p_{1}, \gamma, p_{0}\right) \in \mathrm{G}$ of the connection $A \in \mathcal{A}(P)$ is defined by $p_{1} \rho_{A}\left(p_{1}, \gamma, p_{0}\right):=\tilde{\gamma}(1)$, where $\tilde{\gamma}:[0,1] \rightarrow P$ is the unique horizontal lift of $\gamma$ with $\tilde{\gamma}(0)=p_{0}$. It satisfies

$$
\begin{aligned}
\rho_{A}\left(p_{1} g_{1}, \gamma, p_{0} g_{0}\right) & =g_{1}^{-1} \rho_{A}\left(p_{1}, \gamma, p_{0}\right) g_{0}, \\
\rho_{g^{*} A}\left(p_{1}, \gamma, p_{0}\right) & =g\left(p_{1}\right)^{-1} \rho_{A}\left(p_{1}, \gamma, p_{0}\right) g\left(p_{0}\right),
\end{aligned}
$$

for $g_{0}, g_{1} \in \mathrm{G}$ and $g \in \mathcal{G}$. Hence the map $\mathcal{B} \times \pi^{-1}(\gamma(0)) \rightarrow M$ : $\left(u, A, p_{0}\right) \mapsto \rho_{A}\left(p_{1}, \gamma, p_{0}\right)^{-1} u\left(p_{1}\right)$ is $\mathcal{G}$-invariant and G-equivariant. Choose a finite sequence of smooth functions $\gamma_{i j}:[0,1] \times B_{r}\left(z_{i}\right) \rightarrow \Sigma$ such that

$$
\gamma_{i j}(0, z)=z, \quad \gamma_{i j}(1, z)=z_{j} .
$$

Then the functions $\Theta_{0}: \mathcal{U}_{0} \rightarrow \mathrm{EG}^{m}$ and $\Theta_{i j}: \mathcal{U}_{i j} \rightarrow \mathrm{EG}^{m}$ defined by

$$
\Theta_{0}(u, A, p):=\theta(u(p)), \quad \Theta_{i j}(u, A, p):=\theta\left(\rho_{A}\left(p_{j}, \gamma_{i j}(\cdot, \pi(p)), p\right)^{-1} u\left(p_{j}\right)\right)
$$

for $i, j=1, \ldots, \ell$, satisfy equation (12.1). Now choose a G-invariant smooth function $\beta: M \rightarrow[0,1]$ such that $\beta(x)=1$ for $x \in M^{\delta}$ and $\beta(x)=0$ for $x \in M \backslash M^{\delta+\varepsilon / 2}$. Define $\rho_{j}: \bigcup_{i=1}^{\ell} \mathcal{U}_{i j} \rightarrow[0,1]$ and $\rho_{0}: \mathcal{U}_{0} \rightarrow[0,1]$ by

$$
\begin{aligned}
& \rho_{0}(u, A, p):=\frac{\beta(u(p))}{\sqrt{\beta(u(p))^{2}+(1-\beta(u(p)))^{2}}}, \\
& \rho_{j}(u, A, p):=\frac{\beta\left(u\left(p_{j}\right)\right)(1-\beta(u(p)))}{\sqrt{\left(\beta(u(p))^{2}+(1-\beta(u(p)))^{2}\right) \sum_{k=1}^{\ell} \beta\left(u\left(p_{k}\right)\right)^{2}}}
\end{aligned}
$$

for $j=1, \ldots, \ell$. Then $\rho_{j}$ is smooth with respect to the $C^{0}$ Banach manifold structure on (the completion of) $\mathcal{B} \times P$. Moreover,

$$
\sum_{j=0}^{\ell} \rho_{j}(u, A, p)^{2}=1,
$$

and $\rho_{0}(u, A, p)=1$ whenever $|\mu(u(p))| \leq \delta$. Now choose a finite sequence of smooth functions $\sigma_{i}: \Sigma \rightarrow[0,1], i=1, \ldots, \ell$, such that $\operatorname{supp} \sigma_{i} \subset B_{r}\left(z_{i}\right)$ and $\sum_{j=1}^{\ell} \sigma_{i}^{2}=1$. Then the function $\Theta: \mathcal{B}^{\delta, r} \rightarrow \mathrm{EG}^{\left(\ell^{2}+1\right) m}$, defined by

$$
\Theta(u, A):=\left(\begin{array}{c}
\rho_{0}(u, A, p) \Theta_{0}(u, A, p) \\
\vdots \\
\sigma_{i}(\pi(p)) \rho_{j}(u, A, p) \Theta_{i j}(u, A, p) \\
\vdots
\end{array}\right)
$$

is the required classifying map.

The integer $n=(\ell+1) m$ in the proof of Proposition 12.1 diverges to infinity as $r$ tends to zero. In general, there is no $\mathcal{G}$-equivariant map from 
$\mathcal{B}^{\delta}:=\{(u, A) \in \mathcal{B}|\min | \mu \circ u \mid<\delta\}$ to any finite-dimensional approximation of EG.

Lemma 12.2. Assume (H1) to (H3). Let $B \in H_{2}\left(M_{\mathrm{G}} ; \mathbb{Z}\right),\left(\Sigma, j_{\Sigma}, \mathrm{dvol}_{\Sigma}\right)$ be a compact Riemann surface, $\pi: P \rightarrow \Sigma$ be the principal $\mathrm{G}$-bundle determined by $B$, and $\Sigma \rightarrow \mathcal{J}_{\mathrm{G}}(M, \omega)$ be a smooth family of $\mathrm{G}$-invariant $\omega$-compatible almost complex structures on $M$ such that each $J_{z}$ agrees with the almost complex structure of (H2) outside of a sufficiently large compact subset of $M$. Then for every $\delta>0$, there exist positive constants $r$ and $\varepsilon_{0}$ such that

$$
\widetilde{\mathcal{M}}_{B, \Sigma}^{\varepsilon} \subset \mathcal{B}^{\delta, r}
$$

for $0<\varepsilon \leq \varepsilon_{0}$.

Proof. Suppose the result is false. Then there exist a constant $\delta>0$ and sequences

$$
r_{i} \rightarrow 0, \quad \varepsilon_{i} \rightarrow 0, \quad\left(u_{i}, A_{i}\right) \in \widetilde{\mathcal{M}}_{B, \Sigma}^{\varepsilon_{i}}
$$

such that $\left(u_{i}, A_{i}\right) \notin \mathcal{B}^{\delta, r_{i}}$ for every $i$. This means that, for every $p \in P$, there exists a sequence $p_{i} \in P$ such that

$$
\lim _{i \rightarrow \infty} p_{i}=p, \quad\left|\mu\left(u_{i}\left(p_{i}\right)\right)\right| \geq \delta .
$$

This contradicts the bubbling argument in Step 5 of the proof of Theorem 1.1 below.

Let $\varepsilon_{0}>0$ be as in Lemma 12.2. For $0 \leq \varepsilon \leq \varepsilon_{0}$, we consider the evaluation map

$$
\mathrm{ev}_{\mathrm{G}}^{\varepsilon}: \mathcal{M}_{B, \Sigma}^{\varepsilon} \times \Sigma \longrightarrow M \times_{\mathrm{G}} \mathrm{EG}^{n}
$$

given by

$$
\operatorname{ev}_{\mathrm{G}}^{\varepsilon}([u, A, p]):=[u(p), \Theta(u, A, p)],
$$

where $\delta>0$ is chosen such that $\mathrm{G}_{x}=\{\mathbb{1}\}$ for every $x \in M^{\delta}, r>0$ is as in Lemma 12.2, and $\Theta: \mathcal{B}^{\delta, r} \times P \rightarrow \mathrm{EG}^{n}$ is the map of Proposition 12.1. Recall that $\mathcal{M}_{B, \Sigma}^{0}$ and $\mathcal{M}_{B, \Sigma}^{\varepsilon}$ have the same dimension.

Proposition 12.3. For every $c_{0}>0$ there exist positive constants $c$ and $\varepsilon_{0}$ such that the following holds.

(i) For $0<\varepsilon \leq \varepsilon_{0}$, the map $\mathcal{T}^{\varepsilon}: \mathcal{M}_{B, \Sigma}^{0}\left(c_{0}\right) \rightarrow \mathcal{M}_{B, \Sigma}^{\varepsilon}$ is an orientation preserving embedding.

(ii) For $0<\varepsilon \leq \varepsilon_{0}$,

$$
d_{C^{1}}\left(\operatorname{ev}_{\mathrm{G}}^{0}, \operatorname{ev}_{\mathrm{G}}^{\varepsilon} \circ\left(\mathcal{T}^{\varepsilon} \times \mathrm{id}\right)\right) \leq c \varepsilon^{1-2 / p},
$$

where the $C^{1}$-distance is understood on the space of continuously differentiable maps from $\mathcal{M}_{B, \Sigma}^{0}\left(c_{0}\right) \times \Sigma$ to $M \times_{\mathrm{G}} \mathrm{EG}^{n}$. 
Lemma 12.4. Assume $(\mathrm{H} 1)$ and $(\mathrm{H} 4)$ and let $\bar{B} \in H_{2}(\bar{M} ; \mathbb{Z})$ be a nontorsion homology class. For every $p>2$ and every $c_{0}>0$, there exist positive constants $\varepsilon_{0}$ and $c$ such that the following holds for every $\varepsilon \in\left(0, \varepsilon_{0}\right]$. Let $I \subset \mathbb{R}$ be an interval and

$$
I \longrightarrow \widetilde{\mathcal{M}}_{B, \Sigma}^{0}\left(c_{0}\right): r \longmapsto\left(u_{0}(r), A_{0}(r)\right)
$$

be a smooth path that satisfies equation (8.10). Then every smooth vector field

$$
r \mapsto \zeta(r) \in \operatorname{im}\left(\mathcal{D}_{\left(u_{0}(r), A_{0}(r)\right)}^{\varepsilon}\right)^{*}
$$

satisfies the inequality

$$
\left\|\tilde{\nabla}_{r} \zeta\right\|_{1, p, \varepsilon} \leq c\left(\varepsilon\left\|\tilde{\nabla}_{r} \mathcal{D}^{\varepsilon} \zeta\right\|_{0, p, \varepsilon}+\left\|\tilde{\nabla}_{r} \pi_{u_{0}} \mathcal{D}^{\varepsilon} \zeta\right\|_{L^{p}}+\varepsilon^{-1}\|\zeta\|_{1, p, \varepsilon}\right)
$$

for $r \in I$, where $\mathcal{D}^{\varepsilon}:=\mathcal{D}_{\left(u_{0}(r), A_{0}(r)\right)}^{\varepsilon}$ and $\pi_{u_{0}}$ is defined by equation (2.1).

Proof. Let $r \mapsto \zeta^{\prime}(r) \in \mathcal{X}_{u_{0}(r)}^{\prime}$ be the smooth path defined by $\zeta=\mathcal{D}^{\varepsilon *} \zeta^{\prime}$. Then, by Lemmas $4.7,4.8$, and 8.4 , we have

$$
\begin{aligned}
\left\|\tilde{\nabla}_{r} \zeta\right\|_{1, p, \varepsilon} \leq & \left\|\mathcal{D}^{\varepsilon *} \tilde{\nabla}_{r} \zeta^{\prime}\right\|_{1, p, \varepsilon}+\left\|\tilde{\nabla}_{r} \mathcal{D}^{\varepsilon *} \zeta^{\prime}-\mathcal{D}^{\varepsilon *} \tilde{\nabla}_{r} \zeta^{\prime}\right\|_{1, p, \varepsilon} \\
\leq & c_{2}\left(\varepsilon\left\|\mathcal{D}^{\varepsilon} \mathcal{D}^{\varepsilon *} \tilde{\nabla}_{r} \zeta^{\prime}\right\|_{0, p, \varepsilon}+\left\|\pi_{u_{0}} \mathcal{D}^{\varepsilon} \mathcal{D}^{\varepsilon *} \tilde{\nabla}_{r} \zeta^{\prime}\right\|_{L^{p}}\right) \\
& +\left\|\tilde{\nabla}_{r} \mathcal{D}^{\varepsilon *} \zeta^{\prime}-\mathcal{D}^{\varepsilon *} \tilde{\nabla}_{r} \zeta^{\prime}\right\|_{1, p, \varepsilon} \\
\leq & c_{2}\left(\varepsilon\left\|\mathcal{D}^{\varepsilon} \tilde{\nabla}_{r} \zeta\right\|_{0, p, \varepsilon}+\left\|\pi_{u_{0}} \mathcal{D}^{\varepsilon} \mathcal{D}^{\varepsilon *} \tilde{\nabla}_{r} \zeta^{\prime}\right\|_{L^{p}}\right) \\
& +c_{3}\left\|\tilde{\nabla}_{r} \mathcal{D}^{\varepsilon *} \zeta^{\prime}-\mathcal{D}^{\varepsilon *} \tilde{\nabla}_{r} \zeta^{\prime}\right\|_{1, p, \varepsilon} \\
\leq & c_{2}\left(\varepsilon\left\|\tilde{\nabla}_{r} \mathcal{D}^{\varepsilon} \zeta\right\|_{0, p, \varepsilon}+\left\|\pi_{u_{0}} \mathcal{D}^{\varepsilon} \mathcal{D}^{\varepsilon *} \tilde{\nabla}_{r} \zeta^{\prime}\right\|_{L^{p}}\right) \\
& +c_{2} \varepsilon\left\|\mathcal{D}^{\varepsilon} \tilde{\nabla}_{r} \zeta-\tilde{\nabla}_{r} \mathcal{D}^{\varepsilon} \zeta\right\|_{0, p, \varepsilon}+c_{4} \varepsilon^{-1}\left\|\zeta^{\prime}\right\|_{2, p, \varepsilon} \\
\leq & c_{2}\left(\varepsilon\left\|\tilde{\nabla}_{r} \mathcal{D}^{\varepsilon} \zeta\right\|_{0, p, \varepsilon}+\left\|\tilde{\nabla}_{r} \pi_{u_{0}} \mathcal{D}^{\varepsilon} \zeta\right\|_{L^{p}}\right) \\
& +c_{2}\left\|\left[\pi_{u_{0}} \mathcal{D}^{\varepsilon} \mathcal{D}^{\varepsilon *}, \tilde{\nabla}_{r}\right] \zeta^{\prime}\right\|_{L^{p}}+c_{5} \varepsilon^{-1}\|\zeta\|_{1, p, \varepsilon} \\
\leq & c_{2}\left(\varepsilon\left\|\tilde{\nabla}_{r} \mathcal{D}^{\varepsilon} \zeta\right\|_{0, p, \varepsilon}+\left\|\tilde{\nabla}_{r} \pi_{u_{0}} \mathcal{D}^{\varepsilon} \zeta\right\|_{L^{p}}\right) \\
& +c_{6}\left(\left\|\mathcal{D}^{\varepsilon} \zeta\right\|_{0, p, \varepsilon}+\varepsilon^{-1}\left\|\pi_{u_{0}} \mathcal{D}^{\varepsilon} \zeta\right\|_{L^{p}}+\varepsilon^{-1}\|\zeta\|_{1, p, \varepsilon}\right)
\end{aligned}
$$


The last inequality follows as in Step 7 in the proof of Lemma 4.8. Since

$$
\left\|\mathcal{D}^{\varepsilon} \zeta\right\|_{0, p, \varepsilon}+\varepsilon^{-1}\left\|\pi_{u_{0}} \mathcal{D}^{\varepsilon} \zeta\right\|_{L^{p}} \leq c_{7} \varepsilon^{-1}\|\zeta\|_{1, p, \varepsilon},
$$

the lemma is proved.

Proof of Proposition 12.3. Let $r \mapsto\left(u_{0}(r), A_{0}(r)\right)$ be as in Lemma 12.4 and $r \mapsto \zeta_{\varepsilon}(r)=\left(\xi_{\varepsilon}(r), \alpha_{\varepsilon}(r)\right) \in \operatorname{im}\left(\mathcal{D}_{\left(u_{0}(r), A_{0}(r)\right)}^{\varepsilon}\right)^{*}$ be as in Theorem 6.1 so that

$$
\widetilde{\mathcal{T}}^{\varepsilon}\left(u_{0}(r), A_{0}(r)\right)=\left(u_{\varepsilon}(r), A_{\varepsilon}(r)\right):=\left(\exp _{u_{0}(r)}\left(\xi_{\varepsilon}(r)\right), A_{0}(r)+\alpha_{\varepsilon}(r)\right) .
$$

Let $\mathcal{F}_{r}^{\varepsilon}: \mathcal{X}_{u_{0}(r)} \rightarrow \mathcal{X}_{u_{0}(r)}^{\prime}$ be defined by equation (5.1). Then $\mathcal{F}_{r}^{\varepsilon}\left(\zeta_{\varepsilon}(r)\right)=0$ and hence

$$
\mathcal{D}^{\varepsilon} \zeta_{\varepsilon}=\left(\begin{array}{c}
0 \\
0 \\
* F_{A_{0}(r)}
\end{array}\right)+\left(\mathcal{F}_{r}^{\varepsilon}\left(\zeta_{\varepsilon}(r)\right)-\mathcal{F}_{r}^{\varepsilon}(0)-d \mathcal{F}_{r}^{\varepsilon}(0) \zeta_{\varepsilon}(r)\right) .
$$

Differentiating this identity with respect to $r$, we find

$$
\left\|\tilde{\nabla}_{r} \mathcal{D}^{\varepsilon} \zeta_{\varepsilon}\right\|_{0, p, \varepsilon} \leq c\left(\varepsilon+\varepsilon^{-1-2 / p}\left\|\zeta_{\varepsilon}(r)\right\|_{1, p, \varepsilon}\left(\left\|\zeta_{\varepsilon}(r)\right\|_{1, p, \varepsilon}+\left\|\tilde{\nabla}_{r} \zeta_{\varepsilon}(r)\right\|_{1, p, \varepsilon}\right)\right)
$$

(see Proposition 5.2). Hence, by Lemma 12.4,

$$
\left\|\tilde{\nabla}_{r} \zeta_{\varepsilon}\right\|_{1, p, \varepsilon} \leq c^{\prime} \varepsilon
$$

Since

$$
\left(\partial_{r} u_{\varepsilon}, \partial_{r} A_{\varepsilon}\right)=\left(E_{1}\left(u_{0}, \xi_{\varepsilon}\right) \partial_{r} u_{0}+E_{2}\left(u_{0}, \xi_{\varepsilon}\right) \nabla_{r} \xi_{\varepsilon}, \partial_{r} A_{0}+\partial_{r} \alpha_{\varepsilon}\right),
$$

this shows that $\mathcal{T}^{\varepsilon}: \mathcal{M}_{B, \Sigma}^{0}\left(c_{0}\right) \rightarrow \mathcal{M}_{B, \Sigma}^{\varepsilon}$ is an orientation preserving embedding. Indeed, it follows that the restriction of $\mathcal{T}^{\varepsilon}$ to every ball of radius $\delta$ is an embedding for $\delta$ and $\varepsilon$ sufficiently small and hence, by Theorem 6.1, $\mathcal{T}^{\varepsilon}$ is an embedding for $\varepsilon$ sufficiently small. For $\varepsilon \geq 0$ denote

$$
\mathcal{P}_{B, \Sigma}^{\varepsilon}\left(c_{0}\right):=\widetilde{\mathcal{M}}_{B, \Sigma}^{\varepsilon}\left(c_{0}\right) \times_{\mathcal{G}} P,
$$

and consider the map $\mathrm{ev}^{\varepsilon}: \mathcal{P}_{B, \Sigma}^{\varepsilon}\left(c_{0}\right) \rightarrow M$ defined by

$$
\operatorname{ev}^{\varepsilon}\left(\left[u_{\varepsilon}, A_{\varepsilon}, p\right]\right):=u_{\varepsilon}(p) \text {. }
$$

Then it follows from equation (12.2) and the inequality $\left\|\zeta_{\varepsilon}\right\|_{2, p, \varepsilon} \leq c^{\prime} \varepsilon^{2}$ of Theorem 6.1 that

$$
d_{C^{1}}\left(\mathrm{ev}^{0}, \mathrm{ev}^{\varepsilon} \circ\left(\mathcal{T}^{\varepsilon} \times \mathrm{id}\right)\right) \leq c^{\prime \prime} \varepsilon^{1-2 / p} .
$$

For $\varepsilon$ sufficiently small, we have $\mathrm{ev}_{\mathrm{G}}^{\varepsilon}=\theta^{\delta} \circ \mathrm{ev}^{\varepsilon}$, where $\theta^{\delta}: M^{\delta} \rightarrow M \times_{\mathrm{G}} \mathrm{EG}^{n}$ is given by $\theta^{\delta}(x):=[x, \theta(x)]$. This proves the proposition. 
Proof of Theorem 1.1. The result is obvious when $\bar{B}=0$. Moreover, both moduli spaces are empty when $\bar{B}$ is a nonzero torsion class. Hence assume that $\bar{B} \in H_{2}(\bar{M} ; \mathbb{Z})$ is a nontorsion homology class, denote by $B \in H_{2}\left(M_{\mathrm{G}} ; \mathbb{Z}\right)$ the corresponding equivariant homology class, fix a compact Riemann surface $\left(\Sigma, j_{\Sigma}, \mathrm{dvol}_{\Sigma}\right)$, and let $\pi: P \rightarrow \Sigma$ be a principal Gbundle whose characteristic class $b \in H_{2}(\mathrm{BG} ; \mathbb{Z})$ is the pushforward of $B$. In the course of the proof, it will be necessary to also consider other bundles $\pi^{\prime}: P^{\prime} \rightarrow \Sigma$ with corresponding equivariant homology clsses $B^{\prime} \in H_{2}\left(M_{\mathrm{G}} ; \mathbb{Z}\right)$. By $(\mathrm{H} 2)$, there exists a constant $c>0$ such that $u(P) \subset M^{c}:=\{x \in M|| \mu(x) \mid \leq c\}$ for every solution $(u, A)$ of equation (1.7) over any Riemann surface. Note that $c$ can be chosen to be a regular value of the function $M \rightarrow \mathbb{R}: x \mapsto|\mu(x)|$.

Let $\delta>0$ such that $\mathrm{G}_{x}=\{\mathbb{1}\}$ for every $x \in M^{\delta}$ and let $r$ and $\varepsilon_{0}$ be as in Lemma 12.2. Fix $k$ points $p_{1}, \ldots, p_{k} \in P$ such that the points $z_{i}:=\pi\left(p_{i}\right) \in \Sigma$ are pairwise distinct. Choose an integer $n$, a G-equivariant smooth map $\theta: M^{\delta} \rightarrow \mathrm{EG}^{n}$, and $k$ smooth classifying maps $\Theta_{i}: \mathcal{B}^{\delta, r} \rightarrow \mathrm{EG}^{n}$, defined by $\Theta_{i}(u, A):=\Theta\left(u, A, p_{i}\right)$, where $\Theta$ is as in Proposition 12.1. Then

$$
\Theta_{i}\left(g^{-1} u, g^{*} A\right)=g\left(p_{i}\right)^{-1} \Theta_{i}(u, A),
$$

and

$$
\left|\mu\left(u\left(p_{i}\right)\right)\right| \leq \delta \quad \Longrightarrow \quad \Theta_{i}(u, A)=\theta\left(u\left(p_{i}\right)\right)
$$

for $i=1, \ldots, k$. For $0 \leq \varepsilon \leq \varepsilon_{0}$, consider the evaluation maps

$$
\mathrm{ev}_{B, i}^{\varepsilon}: \mathcal{M}_{B, \Sigma}^{\varepsilon} \rightarrow M_{\mathrm{G}}^{c}:=M^{c} \times_{\mathrm{G}} \mathrm{EG}^{n}
$$

given by $\operatorname{ev}_{B, i}^{\varepsilon}([u, A]):=\left[u\left(p_{i}\right), \Theta_{i}(u, A)\right]$. Let $\operatorname{ev}_{B}^{\varepsilon}: \mathcal{M}_{B, \Sigma}^{\varepsilon} \rightarrow\left(M_{\mathrm{G}}^{c}\right)^{k}$ denote the product map defined by

$$
\operatorname{ev}_{B}^{\varepsilon}([u, A]):=\left(\mathrm{ev}_{B, 1}^{\varepsilon}([u, A]), \ldots, \mathrm{ev}_{B, k}^{\varepsilon}([u, A])\right) .
$$

For any subset $I=\left\{i_{1}, \ldots, i_{j}\right\} \subset\{1, \ldots, k\}$ such that $i_{1}<\cdots<i_{j}$ and any class $B^{\prime} \in H_{2}\left(M_{\mathrm{G}} ; \mathbb{Z}\right)$ that descends to $H_{2}(\bar{M} ; \mathbb{Z})$, we consider the evaluation $\operatorname{map} \operatorname{ev}_{B^{\prime}, I}^{0}: \mathcal{M}_{B^{\prime}, \Sigma}^{0} \rightarrow\left(M_{\mathrm{G}}^{c}\right)^{|I|}$ given by

$$
\mathrm{ev}_{B^{\prime}, I}^{0}([u, A]):=\left(\left[u\left(p_{i_{1}}\right), \theta\left(u\left(p_{i_{1}}\right)\right)\right], \ldots,\left[u\left(p_{i_{j}}\right), \theta\left(u\left(p_{i_{j}}\right)\right)\right]\right) .
$$

Now fix equivariant cohomology classes $\alpha_{1}, \ldots, \alpha_{k} \in H^{*}\left(M_{\mathrm{G}} ; \mathbb{Z}\right)$ such that

$$
m_{i}:=\operatorname{deg}\left(\alpha_{i}\right)<2 N, \quad \sum_{i=1}^{k} \operatorname{deg}\left(\alpha_{i}\right)=\operatorname{dim} \mathcal{M}_{B, \Sigma}^{0} .
$$

There is a natural embedding $M_{\mathrm{G}}^{c} \rightarrow M_{\mathrm{G}}$ and we denote by $\alpha_{i}^{c} \in$ $H^{m_{i}}\left(M_{\mathrm{G}}^{c} ; \mathbb{Z}\right)$ the pullback of $\alpha_{i}$ under this embedding. Note that $M_{\mathrm{G}}^{c}$ is a compact manifold with boundary. Replacing $\alpha_{i}$ by some integer multiple of $\alpha_{i}$, if necessary, we may assume without loss of generality that, for every $i$, there exists a compact oriented manifold with boundary $Y_{i}$ of dimension

$$
\operatorname{dim} Y_{i}=\operatorname{dim} M_{\mathrm{G}}^{c}-m_{i}
$$


and a smooth map

$$
f_{i}:\left(Y_{i}, \partial Y_{i}\right) \rightarrow\left(M_{\mathrm{G}}^{c}, \partial M_{\mathrm{G}}^{c}\right)
$$

such that the homology class in $H_{*}\left(M_{\mathrm{G}}^{c}, \partial M_{\mathrm{G}}^{c}\right)$ represented by $f_{i}$ is Poincaré dual to $\alpha_{i}^{c}$. For $I=\left\{i_{1}, \ldots, i_{j}\right\} \subset\{1, \ldots, k\}$ such that $i_{1}<\cdots<i_{j}$ we denote the corresponding product map by

$$
Y_{I}:=Y_{i_{1}} \times \cdots \times Y_{i_{j}}, \quad f_{I}:=f_{i_{1}} \times \cdots \times f_{i_{j}}: Y_{I} \rightarrow\left(M_{\mathrm{G}}^{c}\right)^{|I|} .
$$

For $I=\{1, \ldots, k\}$ we abbreviate $Y:=Y_{\{1, \ldots, k\}}$ and $f:=f_{\{1, \ldots, k\}}$. The functions $f_{1}, \ldots, f_{k}$ can be chosen such that the following holds.

(H5): $f_{i}$ is transverse to $\mu^{-1}(0) \times_{\mathrm{G}} \mathrm{EG}^{n}$ for every $i$ and $f_{I}$ is transverse to $\mathrm{ev}_{B^{\prime}, I}^{0}$ for every subset $I \subset\{1, \ldots, \ell\}$ and every equivariant homology class $B^{\prime} \in H_{2}\left(M_{\mathrm{G}} ; \mathbb{Z}\right)$.

Now the notation has been set up and we shall prove Theorem 1.1 in five steps. For $0 \leq \varepsilon \leq \varepsilon_{0}$ and $B \in H_{2}\left(M_{\mathrm{G}} ; \mathbb{Z}\right)$ consider the set

$$
\mathcal{M}_{B, \Sigma ; f}^{\varepsilon}:=\left\{\left([u, A], y_{1}, \ldots, y_{k}\right) \in \mathcal{M}_{B, \Sigma}^{\varepsilon} \times Y \mid \operatorname{ev}_{B, i}^{\varepsilon}([u, A])=f_{i}\left(y_{i}\right)\right\} .
$$

Step 1. The map $\operatorname{ev}_{B}^{0}: \mathcal{M}_{B, \Sigma}^{0} \rightarrow\left(\mu^{-1}(0) \times_{\mathrm{G}} \mathrm{EG}^{n}\right)^{k}$ is a pseudo-cycle.

The map $\mathrm{ev}_{B}^{0}$ is the composition $\mathrm{ev}_{B}^{0}=\iota^{k} \circ \mathrm{ev}_{B}$, where the evaluation map $\overline{\mathrm{ev}}_{B}: \mathcal{M}_{B, \Sigma}^{0} \rightarrow \bar{M}^{k}$ is given by $\overline{\mathrm{ev}}_{B}([u, A]):=\left(\left[u\left(p_{1}\right)\right], \ldots,\left[u\left(p_{k}\right)\right]\right)$ and the embedding $\iota: \bar{M} \rightarrow \mu^{-1}(0) \times_{\mathrm{G}} \mathrm{EG}^{n}$ is given by $\iota([x]):=[x, \theta(x)]$. That $\overline{\mathrm{ev}}_{B}$ is a pseudo-cycle was proven in $[\mathbf{1 1}]$. Hence $\mathrm{ev}_{B}^{0}$ is a pseudo-cycle. (see [11] for the definitions).

Step 2. $\mathcal{M}_{B, \Sigma ; f}^{0}$ is a finite set and the number of elements of $\mathcal{M}_{B, \Sigma ; f}^{0}$, counted with appropriate signs, is the Gromov-Witten invariant:

$$
\mathrm{GW}_{\bar{B}, \Sigma}\left(\bar{\alpha}_{1}, \ldots, \bar{\alpha}_{k}\right)=\mathrm{ev}_{B}^{0} \cdot f=\sum_{\left(\left[u_{0}, A_{0}\right], y\right) \in \mathcal{M}_{B, \Sigma ; f}^{0}} \nu^{0}\left(\left[u_{0}, A_{0}\right], y\right) .
$$

Here the function $\nu^{0}: \mathcal{M}_{B, \Sigma ; f}^{0} \rightarrow\{ \pm 1\}$ denotes the intersection index of the maps $\mathrm{ev}_{B}^{0}$ and $f$.

Consider the functions $\varphi_{i}: X_{i} \rightarrow \mu^{-1}(0) \times_{\mathrm{G}} \mathrm{EG}^{n}$ given by

$$
X_{i}:=f_{i}^{-1}\left(\mu^{-1}(0) \times_{\mathrm{G}} \mathrm{EG}^{n}\right) \subset Y_{i}, \quad \varphi_{i}:=\left.f_{i}\right|_{X_{i}} .
$$

Since $f_{i}$ is transverse to $\mu^{-1}(0) \times_{\mathrm{G}} \mathrm{EG}^{n}, X_{i}$ is a smooth submanifold of $Y_{i}$, and $\varphi_{i}$ is dual to the cohomology class $\alpha_{i}^{0} \in H^{*}\left(\mu^{-1}(0) \times_{\mathrm{G}} \mathrm{EG}^{n} ; \mathbb{Z}\right)$ obtained from $\alpha_{i}^{c}$ by pullback under the obvious inclusion $\mu^{-1}(0) \times{ }_{\mathrm{G}} \mathrm{EG}^{n} \rightarrow$ $M_{\mathrm{G}}^{c}$. The class $\alpha_{i}^{0}$ agrees with the image of the class $\bar{\alpha}_{i} \in H^{*}(\bar{M} ; \mathbb{Z})$ under 
the homomorphism $H^{*}(\bar{M} ; \mathbb{Z}) \cong H^{*}\left(\mu^{-1}(0) \times_{\mathrm{G}} \mathrm{EG} ; \mathbb{Z}\right) \rightarrow H^{*}\left(\mu^{-1}(0) \times_{\mathrm{G}}\right.$ $\left.\mathrm{EG}^{n} ; \mathbb{Z}\right):$

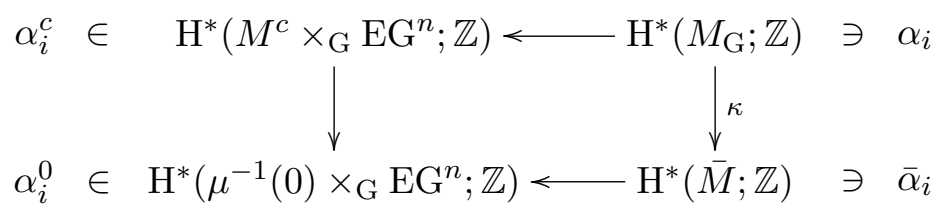

Hence another representative of the class $\alpha_{i}^{0}$ can be obtained as follows. Let $\bar{\psi}_{i}: Z_{i} \rightarrow \bar{M}$ be a smooth function, defined on a compact manifold $\bar{Z}_{i}$ that is dual to $\bar{\alpha}_{i}$ (replace $\bar{\alpha}_{i}$ by an integer multiple of $\bar{\alpha}_{i}$, if necessary). Lift $\bar{\psi}_{i}$ to a G-equivariant map $\tilde{\psi}_{i}: Q_{i} \rightarrow \mu^{-1}(0)$, defined on the total space of a principal G-bundle $Q_{i} \rightarrow Z_{i}$, and consider the induced map

$$
\psi_{i}: Q_{i} \times_{\mathrm{G}} \mathrm{EG}^{n} \rightarrow \mu^{-1}(0) \times_{\mathrm{G}} \mathrm{EG}^{n} .
$$

It is homologous to $\varphi_{i}$. Let $\varphi:=\varphi_{1} \times \cdots \times \varphi_{k}$ and $\psi:=\psi_{1} \times \cdots \times \psi_{k}$. Then

$$
\mathrm{ev}_{B}^{0} \cdot f=\mathrm{ev}_{B}^{0} \cdot \varphi=\mathrm{ev}_{B}^{0} \cdot \psi=\overline{\mathrm{ev}}_{B} \cdot \bar{\psi}=\mathrm{GW}_{\bar{B}, \Sigma}\left(\bar{\alpha}_{1}, \ldots, \bar{\alpha}_{k}\right) .
$$

The first equality follows from the definition of $\varphi$, the second from the fact that $\operatorname{ev}_{B}^{0}$ is a pseudo-cycle (Step 1) and $\varphi$ is homologous to $\psi$, and the last equality follows from the definition of the Gromov-Witten invariants (see [11] for example).

Step 3. The invariant $\Phi_{B, \Sigma}$ can be expressed as the intersection number

$$
\Phi_{B, \Sigma}\left(\alpha_{1} \smile \cdots \smile \alpha_{k}\right)=\mathrm{ev}_{B}^{\varepsilon} \cdot f
$$

for $\varepsilon>0$ sufficiently small.

The map $f: Y \rightarrow\left(M_{\mathrm{G}}^{c}\right)^{k}$ is dual to the class $\pi_{1}^{*} \alpha_{1} \smile \cdots \smile \pi_{k}^{*} \alpha_{k}$, where $\pi_{i}:\left(M_{\mathrm{G}}^{c}\right)^{k} \rightarrow M_{\mathrm{G}}^{c}$ denotes the projection onto the $i$ th factor. Moreover, $\mathrm{ev}_{B, i}^{\varepsilon}=\pi_{i} \circ \mathrm{ev}_{B}^{\varepsilon}$. Hence

$$
\begin{aligned}
\mathrm{ev}_{B}^{\varepsilon} \cdot f & =\int_{\mathcal{M}_{B, \Sigma}^{\varepsilon}}\left(\mathrm{ev}_{B}^{\varepsilon}\right)^{*}\left(\pi_{1}^{*} \alpha_{1}^{c} \smile \cdots \smile \pi_{k}^{*} \alpha_{k}^{c}\right) \\
& =\int_{\mathcal{M}_{B, \Sigma}^{\varepsilon}}\left(\operatorname{ev}_{B, 1}^{\varepsilon}\right)^{*} \alpha_{1}^{c} \smile \cdots \smile\left(\operatorname{ev}_{B, k}^{\varepsilon}\right)^{*} \alpha_{k}^{c} \\
& =\int_{\mathcal{M}_{B, \Sigma}^{\varepsilon}}\left(\iota^{c} \circ \mathrm{ev}_{B, 1}^{\varepsilon}\right)^{*} \alpha_{1} \smile \cdots \smile\left(\iota^{c} \circ \operatorname{ev}_{B, k}^{\varepsilon}\right)^{*} \alpha_{k} \\
& =\int_{\mathcal{M}_{B, \Sigma}^{\varepsilon}} \operatorname{ev}_{\mathrm{G}}^{*}\left(\alpha_{1} \smile \cdots \smile \alpha_{k}\right) .
\end{aligned}
$$

Here $\iota^{c}: M_{\mathrm{G}}^{c} \rightarrow M_{\mathrm{G}}$ denotes the obvious inclusion. The last equality follows from the fact that $\iota^{c} \circ \mathrm{ev}_{B, i}^{\varepsilon}: \mathcal{M}_{B, \Sigma}^{\varepsilon} \rightarrow M_{\mathrm{G}}$ is homotopic to the evaluation map ev $v_{G}$ in the definition of $\Phi_{B, \Sigma}$. 
Step 4. For $\varepsilon>0$ sufficiently small there is an injective map

$$
\mathcal{T}_{B, \Sigma ; f}^{\varepsilon}: \mathcal{M}_{B, \Sigma ; f}^{0} \rightarrow \mathcal{M}_{B, \Sigma ; f}^{\varepsilon}
$$

such that

$$
\mathcal{T}_{B, \Sigma ; f}^{\varepsilon}\left(\left[u_{0}, A_{0}\right], y_{0,1}, \ldots, y_{0, k}\right)=\left(\left[u_{\varepsilon}, A_{\varepsilon}\right], y_{\varepsilon, 1}, \ldots, y_{\varepsilon, k}\right)
$$

satisfies

$$
\begin{gathered}
\left(u_{\varepsilon}, A_{\varepsilon}\right)=\left(\exp _{u_{0}}\left(\xi_{\varepsilon}\right), A_{0}+\alpha_{\varepsilon}\right), \quad\left\|\left(\xi_{\varepsilon}, \alpha_{\varepsilon}\right)\right\|_{2, p, \varepsilon} \leq c \varepsilon^{2-2 / p} \\
\nu^{\varepsilon}\left(\left[u_{\varepsilon}, A_{\varepsilon}\right], y_{\varepsilon, 1}, \ldots, y_{\varepsilon, k}\right)=\nu^{0}\left(\left[u_{0}, A_{0}\right], y_{0,1}, \ldots, y_{0, k}\right) .
\end{gathered}
$$

Here $\nu^{\varepsilon}: \mathcal{M}_{B, \Sigma ; f}^{\varepsilon} \rightarrow\{ \pm 1\}$ denotes the intersection index of the maps $\mathrm{ev}_{B}^{\varepsilon}$ and $f$ (in the transverse case).

Choose $c_{0}>0$ such that $\mathcal{M}_{B, \Sigma ; f}^{0} \subset \mathcal{M}_{B, \Sigma}^{0}\left(c_{0}\right)$ and consider the map

$$
\left(\mathrm{ev}_{B}^{\varepsilon} \circ \mathcal{T}^{\varepsilon}\right) \times f: \mathcal{M}_{B, \Sigma}^{0}\left(c_{0}\right) \times Y \rightarrow M_{\mathrm{G}}^{c} \times M_{\mathrm{G}}^{c} .
$$

By Proposition 12.3 (ii), this map converges to $\mathrm{ev}_{B}^{0} \times f$ in the $C^{1}$-topology as $\varepsilon$ tends to zero. By (H5), the map $\mathrm{ev}_{B}^{0} \times f$ is transverse to the diagonal $\Delta \subset M_{\mathrm{G}}^{c} \times M_{\mathrm{G}}^{c}$. Hence $\left(\mathrm{ev}_{B}^{\varepsilon} \circ \mathcal{T}^{\varepsilon}\right) \times f$ is transverse to $\Delta$ for $\varepsilon$ sufficiently small. Moreover, by Theorem 6.1, the image of $\mathcal{M}_{B, \Sigma ; f}^{0}$ under $\left(\mathrm{ev}_{B}^{\varepsilon} \circ \mathcal{T}^{\varepsilon}\right) \times f$ is $\varepsilon^{2-2 / p}$-close to $\Delta$. Hence, by the implicit function theorem, there is, for $\varepsilon$ sufficiently small, a unique injective map

$$
\mathcal{M}_{B, \Sigma ; f}^{0} \rightarrow\left(\left(\operatorname{ev}_{B}^{\varepsilon} \circ \mathcal{T}^{\varepsilon}\right) \times f\right)^{-1}(\Delta) \subset \mathcal{M}_{B, \Sigma}^{0}\left(c_{0}\right) \times Y
$$

such that the distance between each point and its image is bounded above by a constant times $\varepsilon^{2-2 / p}$. Composing this map with

$$
\mathcal{T}^{\varepsilon} \times \text { id }: \mathcal{M}_{B, \Sigma}^{0} \times Y \rightarrow \mathcal{M}_{B, \Sigma}^{\varepsilon} \times Y
$$

we obtain the required map $\mathcal{T}_{B, \Sigma ; f}^{\varepsilon}$. By Proposition 12.3 (i), the map $\mathcal{T}_{B, \Sigma ; f}^{\varepsilon}$ identifies the two intersection indices.

Step 5. Assume $\Sigma=S^{2}$. Then there exists a constant $\varepsilon_{0}>0$ such that the map $\mathcal{T}_{B, \Sigma ; f}^{\varepsilon}: \mathcal{M}_{B, \Sigma ; f}^{0} \rightarrow \mathcal{M}_{B, \Sigma ; f}^{\varepsilon}$ of Step 4 is surjective for $0<\varepsilon \leq \varepsilon_{0}$.

Suppose, by contradiction, that there exist sequences $\varepsilon_{\nu} \rightarrow 0$ and

$$
\left(\left[u_{\nu}, A_{\nu}\right], y_{1 \nu}, \cdots, y_{k \nu}\right) \in \mathcal{M}_{B, S^{2} ; f}^{\varepsilon_{\nu}}
$$

such that

Consider the sequence

$$
\left(\left[u_{\nu}, A_{\nu}\right], y_{1 \nu}, \cdots, y_{k \nu}\right) \notin \operatorname{im} \mathcal{T}_{B, S^{2} ; f}^{\varepsilon_{\nu}} .
$$

$$
C_{\nu}:=\sup _{P}\left(\left|d_{A_{\nu}} u_{\nu}\right|+\varepsilon_{\nu}^{-1}\left|\mu\left(u_{\nu}\right)\right|\right) .
$$

We prove that $C_{\nu}$ diverges to $\infty$. Assume otherwise that $C_{\nu}$ is bounded. Then, by Theorem 3.3, there exists a constant $c_{1}>0$ such that $\left[u_{\nu}, A_{\nu}\right]$ 
belongs to the image of the map $\mathcal{T}_{B, S^{2}}^{\varepsilon_{\nu}}: \mathcal{M}_{B, S^{2}}^{0}\left(c_{1}\right) \rightarrow \mathcal{M}_{B, S^{2}}^{\varepsilon_{\nu}}$ for $\nu$ sufficiently large. Write

$$
\left(u_{\nu}, A_{\nu}\right)=\widetilde{\mathcal{T}}^{\varepsilon_{\nu}}\left(u_{0 \nu}, A_{0 \nu}\right), \quad\left(u_{0 \nu}, A_{0 \nu}\right) \in \widetilde{\mathcal{M}}_{B, S^{2}}^{0}\left(c_{1}\right) .
$$

Since $\mathcal{M}_{B, S^{2}}^{0}\left(c_{1}\right)$ is compact (it is a moduli space of holomorphic spheres satisfying a uniform bound on their first derivatives), we may assume that the limit

$$
\left(u_{0}, A_{0}\right)=\lim _{\nu \rightarrow \infty}\left(u_{0 \nu}, A_{0 \nu}\right) \in \widetilde{\mathcal{M}}_{B, S^{2}}^{0}\left(c_{1}\right)
$$

exists. Moreover, since $Y$ is compact, we may assume, by passing to a further subsequence if necessary, that the limit

$$
\left(y_{1}, \ldots, y_{k}\right)=\lim _{\nu \rightarrow \infty}\left(y_{1 \nu}, \ldots, y_{k \nu}\right)
$$

exists. Since $\mathrm{ev}_{B}^{\varepsilon_{\nu}} \circ \mathcal{T}^{\varepsilon_{\nu}}$ converges to $\mathrm{ev}_{B}^{0}$ in the $C^{1}$-topology, and

$$
\mathrm{ev}_{B}^{\varepsilon_{\nu}} \circ \mathcal{T}^{\varepsilon_{\nu}}\left(\left[u_{0 \nu}, A_{0 \nu}\right]\right)=f\left(y_{1 \nu}, \cdots, y_{k \nu}\right)
$$

we deduce that $\left(\left[u_{0}, A_{0}\right], y_{1}, \ldots, y_{k}\right) \in \mathcal{M}_{B, S^{2} ; f}^{0}$ and, for $\nu$ sufficiently large,

$$
\left(\mathcal{T}^{\varepsilon_{\nu}}\left(\left[u_{0 \nu}, A_{0 \nu}\right]\right), y_{1 \nu}, \cdots, y_{k \nu}\right)=\mathcal{T}_{B, S^{2} ; f}^{\varepsilon_{\nu}}\left(\left[u_{0}, A_{0}\right], y_{1}, \ldots, y_{k}\right) .
$$

The last assertion follows from the uniqueness part of the implicit function theorem used in the definition of the maps $\mathcal{T}_{B, S^{2} ; f}^{\varepsilon_{\nu}}$. This contradicts our assumption. Thus we have proved that $C_{\nu}$ diverges to $\infty$ as claimed.

Now choose a sequence $p_{\nu} \in P$ such that

$$
c_{\nu}:=\left|d_{A_{\nu}} u_{\nu}\left(p_{\nu}\right)\right|+\varepsilon_{\nu}^{-1}\left|\mu\left(u_{\nu}\left(p_{\nu}\right)\right)\right| \rightarrow \infty .
$$

Passing to a subsequence, if necessary, we may assume that $p_{\nu}$ converges. Denote

$$
\mathrm{w}:=\lim _{\nu \rightarrow \infty} \pi\left(p_{\nu}\right)
$$

Moreover, by applying Hofer's trick (see [11, Lemma 4.5.3] for example) we may assume that

$$
\sup _{B_{r_{\nu}}\left(\pi\left(p_{\nu}\right)\right)}\left(\left|d_{A_{\nu}} u_{\nu}\right|+\varepsilon_{\nu}^{-1}\left|\mu\left(u_{\nu}\right)\right|\right) \leq 2 c_{\nu}, \quad r_{\nu} c_{\nu} \rightarrow \infty .
$$

We distinguish three cases.

Case 1: $c_{\nu} \varepsilon_{\nu} \rightarrow \infty$.

Case 2: There exists a $\delta>0$ such that $\delta \leq c_{\nu} \varepsilon_{\nu} \leq \delta^{-1}$ for all $\nu$.

Case 3: $c_{\nu} \varepsilon_{\nu} \rightarrow 0$.

We shall prove that in Case 1 a nonconstant $J$-holomorphic sphere in $M$ bubbles off at the point $\mathrm{w}$, in Case 2 a nontrivial solution of the vortex equations (11.1) bubbles off, and in Case 3 a nonconstant $\bar{J}$-holomorphic sphere in $\bar{M}$ bubbles off. To see this, we choose a local holomorphic coordinate chart $s+i t$ on $\Sigma$ that maps w to zero, identifies a neighbourhood of $\mathrm{w}$ with the ball $B_{2 r}$, and identifies the volume form dvol $_{\Sigma}$ with the form 
$\lambda^{2} d s \wedge d t$, where $\lambda(0)=1$. Moreover, we choose a local frame of the bundle $P$ along this coordinate chart. We use the notation of Remark 4.1. Then the sequences $u_{\nu}: B_{2 r} \rightarrow M$ and $\Phi_{\nu}, \Psi_{\nu}: B_{2 r} \rightarrow \mathfrak{g}$ satisfy

$$
\begin{gathered}
v_{\nu s}+J v_{\nu t}=0, \quad \lambda^{-2} \kappa_{\nu}+\varepsilon_{\nu}^{-2} \mu\left(u_{\nu}\right)=0, \\
v_{\nu s}:=\partial_{s} u_{\nu}+X_{\Phi_{\nu}}\left(u_{\nu}\right), \quad v_{\nu t}:=\partial_{t} u_{\nu}+X_{\Psi_{\nu}}\left(u_{\nu}\right), \\
\kappa_{\nu}:=\partial_{s} \Psi_{\nu}-\partial_{t} \Phi_{\nu}+\left[\Phi_{\nu}, \Psi_{\nu}\right] .
\end{gathered}
$$

Moreover, there is a sequence $\mathrm{w}_{\nu}:=\left(s_{\nu}, t_{\nu}\right) \rightarrow 0$ such that

$$
\begin{aligned}
c_{\nu} & =\lambda\left(\mathrm{w}_{\nu}\right)^{-1}\left|v_{\nu s}\left(\mathrm{w}_{\nu}\right)\right|+\varepsilon_{\nu}^{-1}\left|\mu\left(u\left(\mathrm{w}_{\nu}\right)\right)\right| \\
& \geq \frac{1}{2} \sup _{B_{r_{\nu}}\left(\mathrm{w}_{\nu}\right)}\left(\lambda^{-1}\left|v_{\nu s}\right|+\varepsilon_{\nu}^{-1}\left|\mu\left(u_{\nu}\right)\right|\right) .
\end{aligned}
$$

Let us define $\tilde{u}_{\nu}: B_{r_{\nu} c_{\nu}} \rightarrow M$ and $\tilde{\Phi}_{\nu}, \tilde{\Psi}_{\nu}: B_{r_{\nu} c_{\nu}} \rightarrow \mathfrak{g}$ by

$$
\begin{gathered}
\tilde{u}_{\nu}(z):=u\left(\mathrm{w}_{\nu}+c_{\nu}^{-1} z\right), \\
\tilde{\Phi}_{\nu}(z):=c_{\nu}^{-1} \Phi_{\nu}\left(\mathrm{w}_{\nu}+c_{\nu}^{-1} z\right), \quad \tilde{\Psi}_{\nu}(z):=c_{\nu}^{-1} \Psi_{\nu}\left(\mathrm{w}_{\nu}+c_{\nu}^{-1} z\right),
\end{gathered}
$$

and $\tilde{\lambda}_{\nu}: B_{r_{\nu} c_{\nu}} \rightarrow(0, \infty)$ and $\tilde{J}_{\nu}: B_{r_{\nu} c_{\nu}} \rightarrow \mathcal{J}_{\mathrm{G}}(M, \omega)$ by

$$
\tilde{\lambda}_{\nu}(z):=\lambda\left(\mathrm{w}_{\nu}+c_{\nu}^{-1} z\right), \quad \tilde{J}_{\nu}(z)=J_{\mathrm{w}_{\nu}+c_{\nu}^{-1} z} .
$$

Then $\tilde{\lambda}_{\nu}$ converges to 1 in the $C^{\infty}$-topology and $\tilde{J}_{\nu}$ converges to $J_{0}$ in the $C^{\infty}$-topology. Moreover,

$$
\begin{aligned}
& \tilde{v}_{\nu s}+\tilde{J}_{\nu} \tilde{v}_{\nu t}=0, \quad \tilde{\lambda}_{\nu}^{-2} \tilde{\kappa}_{\nu}+\left(c_{\nu} \varepsilon_{\nu}\right)^{-2} \mu\left(\tilde{u}_{\nu}\right)=0, \\
& \sup _{B_{r_{\nu} c_{\nu}}}\left(\frac{1}{\tilde{\lambda}_{\nu}}\left|\tilde{v}_{\nu s}\right|+\frac{1}{c_{\nu} \varepsilon_{\nu}}\left|\mu\left(\tilde{u}_{\nu}\right)\right|\right) \leq 2\left(\frac{1}{\tilde{\lambda}_{\nu}(0)}\left|\tilde{v}_{\nu s}(0)\right|+\frac{1}{c_{\nu} \varepsilon_{\nu}}\left|\mu\left(\tilde{u}_{\nu}(0)\right)\right|\right)=2 .
\end{aligned}
$$

Case 1. Suppose that $c_{\nu} \varepsilon_{\nu}$ diverges to infinity. Then, by hypothesis (H2), the curvature $\tilde{\kappa}_{\nu}$ converges uniformly to zero. Hence, by Uhlenbeck's weak compactness theorem $[\mathbf{1 7}, \mathbf{1 8}]$, we may assume that $\tilde{\Phi}_{\nu}$ and $\tilde{\Psi}_{\nu}$ converge in the sup-norm and weakly in $W^{1, p}$. This implies that the sequence $\tilde{u}_{\nu}$ is bounded in $W^{1, p}$. Hence, by the usual elliptic bootstrapping argument for pseudoholomorphic curves, it is bounded in $W^{2, p}$ (the lower-order terms in the equation have the form $X_{\tilde{\Phi}_{\nu}}\left(\tilde{u}_{\nu}\right)$ and hence are bounded, in $\left.W^{1, p}\right)$. Hence there exists a subsequence, still denoted by $\tilde{u}_{\nu}$, that converges strongly in $W^{1, p}$ to a $J_{0}$-holomorphic curve $\tilde{u}: \mathbb{C} \rightarrow M$ with finite energy. Since the sequence $\mu\left(\tilde{u}_{i}(0)\right)$ is bounded, it follows that $\left|\partial_{s} \tilde{u}(0)\right|=\lim _{\nu \rightarrow \infty}\left|\tilde{v}_{\nu s}(0)\right|=1$, and hence $\tilde{u}$ extends to a nonconstant holomorphic sphere in $M$. This contradicts (H2).

Case 2. Suppose that the sequence $c_{\nu} \varepsilon_{\nu}$ is bounded and does not converge to zero. Let us assume, without loss of generality, that $\lim _{\nu \rightarrow \infty} c_{\nu} \varepsilon_{\nu}=1$. Then we can use the compactness result of [2] to deduce that, after a suitable gauge transformation and after passing to a further subsequence, the triple 
$\left(\tilde{u}_{\nu}, \tilde{\Phi}_{\nu}, \tilde{\Psi}_{\nu}\right)$ converges to a solution $(\tilde{u}, \tilde{\Phi}, \tilde{\Psi})$ of the vortex equations $(11.1)$ with finite energy. Moreover,

$$
\left|\partial_{s} \tilde{u}(0)+X_{\tilde{\Phi}(0)}(\tilde{u}(0))\right|+|\mu(\tilde{u}(0))|=1,
$$

and hence the energy is nonzero. Hence, by Proposition 11.1, we have

$$
E(\tilde{u}, \tilde{\Phi}, \tilde{\Psi}) \geq \hbar
$$

Case 3. Suppose that $\lim _{\nu \rightarrow \infty} c_{\nu} \varepsilon_{\nu}=0$. Then, by Lemma 9.1,

$$
\sup _{\nu}\left(c_{\nu} \varepsilon_{\nu}\right)^{2 / p-2}\left\|\mu\left(\tilde{u}_{\nu}\right)\right\|_{L^{\infty}(K)}+\sup _{\nu}\left(c_{\nu} \varepsilon_{\nu}\right)^{-2}\left\|\mu\left(\tilde{u}_{\nu}\right)\right\|_{L^{p}(K)}<\infty
$$

for every compact set $K \subset \mathbb{C}$ and every $p \geq 2$. Fix a constant $p>2$. Then the sequence $\tilde{\kappa}_{i}$ is uniformly bounded in $L^{p}$. Hence, by Uhlenbeck's weak compactness theorem, we may assume that $\tilde{\Phi}_{\nu}$ and $\tilde{\Psi}_{\nu}$ converge, weakly in $W^{1, p}$ and strongly in $L^{\infty}$, on every compact subset of $\mathbb{C}$. Hence it follows from the elliptic bootstrapping analysis for pseudoholomorphic curves (see [11, Appendix B]) that $\tilde{u}_{\nu}$ is bounded in $W^{2, p}$. Hence, by the ArzélaAscoli theorem, $\tilde{u}_{\nu}$ has a subsequence that converges in the $C^{1}$-norm on every compact subset of $\mathbb{C}$. The limit $(\tilde{u}, \tilde{\Phi}, \tilde{\Psi})$ is a finite energy solution of equation $(1.8)$ on $\mathbb{C}$. This solution represents a $\bar{J}$-holomorphic sphere in the quotient $\bar{M}$. Moreover, since $\left(c_{\nu} \varepsilon_{\nu}\right)^{-1}\left|\mu\left(\tilde{u}_{\nu}(0)\right)\right| \rightarrow 0$ it follows that

$$
\left|\partial_{s} \tilde{u}(0)+X_{\tilde{\Phi}(0)}(\tilde{u}(0))\right|=\lim _{\nu \rightarrow \infty}\left|\partial_{s} \tilde{u}_{\nu}(0)+X_{\tilde{\Phi}_{\nu}(0)}\left(\tilde{u}_{\nu}(0)\right)\right|=1,
$$

and hence the resulting holomorphic sphere in $\bar{M}$ is nonconstant. Hence

$$
E(\tilde{u}, \tilde{\Phi}, \tilde{\Psi}) \geq \hbar \text {. }
$$

Thus we have proved in all three cases that

$$
\lim _{\nu \rightarrow \infty} E_{B_{r}(\mathrm{w})}\left(u_{\nu}, A_{\nu}\right) \geq \hbar
$$

for every $r>0$.

This shows that, after passing to a suitable subsequence, bubbling can only take place at finitely many points $\mathrm{w}_{1}, \ldots, \mathrm{w}_{\ell} \in \Sigma$. On every compact subset of $\Sigma \backslash\left\{\mathrm{w}_{1}, \ldots, \mathrm{w}_{\ell}\right\}$, the sequence $\left|d_{A_{\nu}} u_{\nu}\right|+\varepsilon_{\nu}^{-1}\left|\mu\left(u_{\nu}\right)\right|$ is uniformly bounded. (As an aside: this is used in the proof of Lemma 12.2.) Hence it follows as in Case 3 that a suitable subsequence in a suitable gauge converges on this complement to a finite energy solution of equation (1.8). The limit $(u, A)$ descends to a holomorphic curve

$$
\bar{u}: \Sigma \backslash\left\{\mathrm{w}_{1}, \ldots, \mathrm{w}_{\ell}\right\} \rightarrow \bar{M}
$$

with finite energy. Hence, by the removable singularity theorem for $\bar{J}$-holomorphic curves, it extends to a holomorphic curve on all of $\Sigma$, still denoted by $\bar{u}$. The energy of this $\bar{J}$-holomorphic curve satisfies

$$
E(\bar{u}) \leq\langle[\bar{\omega}], \bar{B}\rangle-\ell \hbar .
$$


By hypothesis (H3), the dimension of the moduli space reduces by at least $2 N$ at each bubble. Thus the limit $[u, A]$ belongs to a moduli space $\mathcal{M}_{B^{\prime}, S^{2}}^{0}$ of dimension

$$
\operatorname{dim} \mathcal{M}_{B^{\prime}, S^{2}}^{0} \leq \operatorname{dim} \mathcal{M}_{B, S^{2}}^{0}-2 N \ell .
$$

If $\left\{\mathrm{w}_{1}, \ldots, \mathrm{w}_{\ell}\right\} \cap\left\{z_{1}, \ldots, z_{k}\right\}=\emptyset$, then the limit curve $(u, A)$ still satisfies $\operatorname{ev}_{B^{\prime}, i}^{0}([u, A]) \in f_{i}\left(Y_{i}\right)$ for every $i$ and hence cannot exist, by the transversality condition (H5). In general, denote

$$
I:=\left\{i \in\{1, \ldots, k\} \mid z_{i} \notin\left\{\mathrm{w}_{1}, \ldots, \mathrm{w}_{\ell}\right\}\right\} .
$$

Then the limit $[u, A]$ satisfies

$$
i \in I \quad \Longrightarrow \quad \mathrm{ev}_{B^{\prime}, i}^{0}([u, A]) \in f_{i}\left(Y_{i}\right) .
$$

Since the points $z_{i} \in \Sigma$ are pairwise distinct, we have

$$
\ell \geq k-|I|,
$$

and so

$$
\operatorname{dim} \mathcal{M}_{B^{\prime}, S^{2}}^{0} \leq \operatorname{dim} \mathcal{M}_{B, S^{2}}^{0}-2 N(k-|I|)<\sum_{i \in I} \operatorname{deg}\left(\alpha_{i}\right)
$$

Here we have used the fact that $\operatorname{deg}\left(\alpha_{i}\right)<2 N$ for each $i \in\{1, \ldots, k\} \backslash I$. It follows again from (H5) that such a limit curve cannot exist. Hence our assumption that the map $\mathcal{T}_{B, S^{2} ; f}^{\varepsilon_{i}}$ was not surjective for every $i$ must have been wrong. This proves the theorem.

Remark 12.5. A more subtle argument, as in Gromov compactness for pseudoholomorphic spheres, shows that in the higher genus case the limit curve $\bar{u}$ also satisfies

$$
\left\langle c_{1}(T \bar{M}),[\bar{u}]\right\rangle \leq\left\langle c_{1}(T \bar{M}), \bar{B}\right\rangle-N \ell,
$$

where $\ell$ denotes the number of points near which bubbling occurs. Here one needs to prove that no energy gets lost and one obtains convergence to a bubble tree that represents the homology class $B$. With this refined compactness argument, one can extend Theorem 1.1 to the higher genus case.

Remark 12.6. The more subtle compactness argument of Remark 12.5 is not needed if we impose the condition $[\omega-\mu]=\tau c_{1}^{\mathrm{G}}(T M)$ instead of (H3). Hence, in this case, Theorem 1.1 continues to hold for Riemann surfaces $\Sigma$ of arbitrary genus.

Remark 12.7. Assume (H1), (H2), and (H4), but not the monotonicity hypothesis (H3). Suppose that the number $\hbar>0$ is a lower bound for the energy of the nonconstant $\bar{J}$-holomorphic spheres in $\bar{M}$ as well as for the energy of the nontrivial (i.e. is positive energy) solutions of the vortex 
equations (11.1). Let $\left(\Sigma, \mathrm{dvol}_{\Sigma}, j_{\Sigma}\right)$ be a compact Riemann surface of genus $g>0$ and suppose that $\bar{B} \in H_{2}(\bar{M} ; \mathbb{Z})$ satisfies

$$
0 \leq\langle[\bar{\omega}], \bar{B}\rangle<\hbar \text {. }
$$

Then the moduli space $\mathcal{M}_{B, \Sigma}^{0}$ is compact and the bubbling argument in the proof of Theorem 1.1 together with Proposition 12.3 shows that the map $\mathcal{T}^{\varepsilon}: \mathcal{M}_{B, \Sigma}^{0} \rightarrow \mathcal{M}_{B, \Sigma}^{\varepsilon}$ of Theorem 6.1 is a diffeomorphism for $\varepsilon>0$ sufficiently small. Hence in this case the invariants $\Phi_{B, \Sigma}$ agree with the Gromov-Witten invariants $\mathrm{GW}_{\bar{B}, \Sigma}$.

\section{Appendix A. The graph construction}

Let $G$ be a compact Lie group whose Lie algebra $\mathfrak{g}=\operatorname{Lie}(G)$ is equipped with an invariant inner product and $(M, \omega)$ be a symplectic manifold with a Hamiltonian G-action generated by a moment map $\mu: M \rightarrow \mathfrak{g}$. We denote by $\mathfrak{g} \rightarrow \operatorname{Vect}(M): \eta \mapsto X_{\eta}$ the infinitesimal action, by $C_{\mathrm{G}}^{\infty}(M)$ the space of G-invariant smooth functions on $M$, and by $\mathcal{J}_{\mathrm{G}}(M, \omega)$ the space of G-invariant and $\omega$-compatible almost complex structures on $M$. We fix a Riemann surface $\left(\Sigma, \operatorname{dvol}_{\Sigma}, j_{\Sigma}\right)$ and a principal G-bundle $P \rightarrow \Sigma$. Given a family of almost complex structures $\Sigma \rightarrow \mathcal{J}_{\mathrm{G}}(M, \omega): z \mapsto J_{z}$ and a 1-form $T \Sigma \rightarrow C_{\mathrm{G}}^{\infty}(M): \hat{z} \mapsto H_{\hat{z}}$, we consider the perturbed equations

$$
\bar{\partial}_{J, H, A}(u)=0, \quad * F_{A}+\varepsilon^{-2} \mu(u)=0,
$$

where

$$
\bar{\partial}_{J, H, A}(u):=\bar{\partial}_{J, A}(u)+X_{H}(u)^{0,1} .
$$

Here the $(0,1)$-form $\bar{\partial}_{J, A}(u) \in \Omega^{0,1}\left(\Sigma, u^{*} T M / \mathrm{G}\right)$ is understood with respect to the family of almost complex structures $J_{z}$, parametrized by $z \in \Sigma$. Moreover, the Hamiltonian perturbation is defined as follows. Associated to $H \in \Omega^{1}\left(\Sigma, C_{\mathrm{G}}^{\infty}(M)\right)$ is the 1 -form $X_{H} \in \Omega^{1}\left(\Sigma, \operatorname{Vect}_{\mathrm{G}}(M, \omega)\right)$ which assigns to every $\hat{z} \in T_{z} \Sigma$ the Hamiltonian vector field $X_{H, \hat{z}}$ associated to the Hamiltonian function $H_{\hat{z}}: M \rightarrow \mathbb{R}$. Thus $\iota\left(X_{H, \hat{z}}\right) \omega=d H_{\hat{z}}$. The 1 -form $X_{H}(u) \in \Omega^{1}\left(\Sigma, u^{*} T M / \mathrm{G}\right)$ lifts to an equivariant and horizontal 1-form on $P$ with values in $u^{*} T M$, also denoted by $X_{H}(u)$ and defined by

$$
\left(X_{H}(u)\right)_{p}(v):=X_{H, d \pi(p) v}(u(p)) .
$$

The complex anti-linear part of this 1-form is the Hamiltonian term in the definition of $\bar{\partial}_{J, H, A}(u)$. In this section, we show how to reduce the perturbed equations (A.1) to (1.7) via Gromov's graph construction [9].

Let us denote by $\alpha_{H} \in \Omega^{1}(\Sigma \times M)$ the 1 -form associated to $H$. Thus $\alpha_{H}$ assigns to every pair of tangent vectors $(\hat{z}, \hat{x}) \in T_{z} \Sigma \times T_{x} M$ the real number $H_{\hat{z}}(x)$. Denote

$$
\tilde{M}:=\Sigma \times M
$$

The 2-form

$$
\tilde{\omega}:=\omega-d \alpha_{H}+c \mathrm{dvol}_{\Sigma}
$$


is a symplectic form on $\tilde{M}$ whenever the constant $c$ is sufficiently large. Here we have abused notation and denoted by $\omega$ the pullback of the 2-form $\omega$ on $M$ under the obvious projection $\Sigma \times M \rightarrow M$ and likewise for $\operatorname{dvol}_{\Sigma}$. To see that $\tilde{\omega}$ is symplectic for large $c$, note first that $\tilde{\omega}$ is a connection form: it is closed and its restriction to each fibre $\{z\} \times M$ is symplectic. The curvature of this connection form is the 2 -form

$$
\Omega_{H} \operatorname{dvol}_{\Sigma}:=d H+\frac{1}{2}\{H \wedge H\} \in \Omega^{2}\left(\Sigma, C_{\mathrm{G}}^{\infty}(M)\right) .
$$

This identity defines the function $\Omega_{H}: \Sigma \times M \rightarrow \mathbb{R}$. Now the top exterior power of $\tilde{\omega}$ is given by

$$
\frac{\tilde{\omega}^{n+1}}{(n+1) !}=\left(c-\Omega_{H}\right) \frac{\omega^{n}}{n !} \wedge \operatorname{dvol}_{\Sigma}
$$

where $\operatorname{dim} M=2 n$. Hence $\tilde{\omega}$ is nondegenerate whenever $c>\max \Omega_{H}$. Now consider the almost complex structure $\tilde{J}$ on $\tilde{M}$ given by

$$
\tilde{J}(z, x):=\left(\begin{array}{cc}
j_{\Sigma}(z) & 0 \\
J(z, x) \circ X_{H}(z, x)-X_{H}(z, x) \circ j_{\Sigma}(z) & J(z, x)
\end{array}\right) .
$$

Here $J(z, x):=J_{z}(x)$ and we denote by $X_{H}(z, x): T_{z} \Sigma \rightarrow T_{x} M$ the linear map $\hat{z} \mapsto X_{H, \hat{z}}(x)$. Lemma A.2 shows that $\tilde{J}$ is compatible with $\tilde{\omega}$.

Lemma A.1. Let $(u, A) \in C_{\mathrm{G}}^{\infty}(P, M) \times \mathcal{A}(P)$ and define $\tilde{u}: P \rightarrow \tilde{M}$ by $\tilde{u}(p):=(\pi(p), u(p))$. Then $u$ and $A$ satisfy equations (A.1) if and only if $\tilde{u}$ and $A$ satisfy

$$
\bar{\partial}_{\tilde{J}, A}(\tilde{u})=0, \quad * F_{A}+\varepsilon^{-2} \tilde{\mu}(\tilde{u})=0 .
$$

Here $\tilde{\mu}: \tilde{M} \rightarrow \mathfrak{g}$ is defined by $\tilde{\mu}(z, x):=\mu(x)$.

Proof. By definition of $\tilde{J}$ we have $\bar{\partial}_{\tilde{J}, A}(\tilde{u})=\left(0, \bar{\partial}_{J, H, A}(u)\right)$. Alternatively, we can compute in local holomorphic coordinates, $s+i t$ on $\Sigma$. In such coordinates, the Hamiltonian perturbation, the connection $A$, and the volume form on $\Sigma$ have the form

$$
H=F d s+G d t, \quad A=\Phi d s+\Psi d t, \quad \operatorname{dvol}_{\Sigma}=\lambda^{2} d s \wedge d t,
$$

and the equations (A.1) have the form

$$
\begin{aligned}
\partial_{s} u+L_{u} \Phi+X_{F}(u)+J\left(\partial_{t} u+L_{u} \Psi+X_{G}(u)\right) & =0, \\
\partial_{s} \Psi-\partial_{t} \Phi+[\Phi, \Psi]+\left(\frac{\lambda}{\varepsilon}\right)^{2} \mu(u) & =0 .
\end{aligned}
$$

Moreover, the almost complex structure $\tilde{J}$ is given by

$$
\tilde{J}:=\left(\begin{array}{ccc}
0 & -1 & 0 \\
1 & 0 & 0 \\
J X_{F}-X_{G} & J X_{G}+X_{F} & J
\end{array}\right) .
$$

This proves the lemma. 
Lemma A.2. Let $\hat{z}_{i} \in T_{z} \Sigma$ and $\hat{x}_{i} \in T_{x} M$ for $i=1,2$. Then

$$
\tilde{\omega}\left(\left(\hat{z}_{1}, \hat{x}_{1}\right),\left(\hat{z}_{2}, \hat{x}_{2}\right)\right)=\left\langle\hat{x}_{1}+X_{H, \hat{z}_{1}}(x), \hat{x}_{2}+X_{H, \hat{z}_{2}}(x)\right\rangle_{z}+\left(c-\Omega_{H}\right)\left\langle\hat{z}_{1}, \hat{z}_{2}\right\rangle,
$$

where $\langle\cdot, \cdot\rangle_{z}:=\omega\left(\cdot, J_{z} \cdot\right)$.

Proof. Continue the notation of the proof of Lemma A.1. Then the curvature $\Omega_{H}$ is given by

$$
\lambda^{2} \Omega_{H}=\partial_{s} G-\partial_{t} F+\{F, G\},
$$

where $\{F, G\}:=\omega\left(X_{F}, X_{G}\right)$ denotes the Poisson bracket on $M$, and

$$
\tilde{\omega}=\omega-d F \wedge d s-d G \wedge d t+\left(\partial_{t} F-\partial_{s} G+c \lambda^{2}\right) d s \wedge d t,
$$

where $d F$ and $d G$ denote the differential on $M$. Abbreviate $\zeta_{i}:=\left(\hat{s}_{i}, \hat{t}_{i}, \hat{x}_{i}\right)$ and $\xi_{i}:=\hat{x}_{i}+\hat{s}_{i} X_{F}+\hat{t}_{i} X_{G}$ for $i=1,2$. Then

$$
\begin{aligned}
\tilde{\omega}\left(\zeta_{1}, \tilde{J} \zeta_{2}\right)= & \tilde{\omega}\left(\zeta_{1},\left(-\hat{t}_{2}, \hat{s}_{2}, J \xi_{2}+\hat{t}_{2} X_{F}-\hat{s}_{2} X_{G}\right)\right) \\
= & \omega\left(\hat{x}_{1}, J \xi_{2}+\hat{t}_{2} X_{F}-\hat{s}_{2} X_{G}\right) \\
& +\hat{t}_{2} d F\left(\hat{x}_{1}\right)+\hat{s}_{1} d F\left(J \xi_{2}+\hat{t}_{2} X_{F}-\hat{s}_{2} X_{G}\right) \\
& -\hat{s}_{2} d G\left(\hat{x}_{1}\right)+\hat{t}_{1} d G\left(J \xi_{2}+\hat{t}_{2} X_{F}-\hat{s}_{2} X_{G}\right) \\
& +\left(\partial_{t} F-\partial_{s} G+c \lambda^{2}\right)\left(\hat{s}_{1} \hat{s}_{2}+\hat{t}_{1} \hat{t}_{2}\right) \\
= & \omega\left(\xi_{1}, J \xi_{2}\right)+\left(c \lambda^{2}-\partial_{s} G+\partial_{t} F-\{F, G\}\right)\left(\hat{s}_{1} \hat{s}_{2}+\hat{t}_{1} \hat{t}_{2}\right) .
\end{aligned}
$$

The last identity follows from the fact that $\{F, G\}=d F\left(X_{G}\right)=-d G\left(X_{F}\right)$ and $d F\left(J \xi_{2}\right)=\omega\left(X_{F}, J \xi_{2}\right)$.

\section{Appendix B. Cauchy-Riemann operators}

Fix a compact Lie group $\mathrm{G}$, an invariant inner product on the Lie algebra $\mathfrak{g}=\operatorname{Lie}(\mathrm{G})$, a symplectic manifold $(M, \omega)$, a Hamiltonian G-action on $M$ generated by a moment map $\mu: M \rightarrow \mathfrak{g}$, a compact Riemann surface $\left(\Sigma, j_{\Sigma}, \mathrm{dvol}_{\Sigma}\right)$, a principal G-bundle $P \rightarrow \Sigma$, and a family of G-invariant and $\omega$-compatible almost complex structures $\Sigma \rightarrow \mathcal{J}_{\mathrm{G}}(M, \omega): z \mapsto J_{z}$. Each almost complex structure determines a Riemannian metric $\langle\cdot, \cdot\rangle_{z}:=\omega\left(\cdot, J_{z} \cdot\right)$ on $M$ and hence a Levi-Civita connection $\nabla=\nabla_{z}$. The value of $z$ will usually be clear from the context and we shall omit the subscript $z$. Let $u: P \rightarrow M$ be an equivariant smooth map and $A$ be a connection on $P$. Then $A$ and $\nabla$ determine a connection $\nabla_{A}$ on $u^{*} T M / \mathrm{G}$ given by

$$
\nabla_{A} \xi:=\nabla \xi+\nabla_{\xi} X_{A}(u)
$$

for $\xi \in \Omega^{0}\left(\Sigma, u^{*} T M / \mathrm{G}\right)$. More precisely, we think of $A$ as an equivariant 1 -form on $P$ with values in the Lie algebra $\mathfrak{g}$ which identifies the vertical tangent bundle with $\mathfrak{g}$. A section $\xi$ of $u^{*} T M / \mathrm{G}$ lifts to an equivariant section of the bundle $u^{*} T M \rightarrow P$ (also denoted by $\xi$ ) and a 1-form $\theta \in$ $\Omega^{1}\left(\Sigma, u^{*} T M / \mathrm{G}\right)$ lifts to an equivariant and horizontal 1-form on $P$ with 
values in $u^{*} T M$ (also denoted by $\theta$ ). In this notation, the 1 -form $\nabla_{A} \xi$ is given by

$$
\left(\nabla_{A} \xi\right)_{p}(v):=\nabla_{v} \xi(p)+\nabla_{\xi(p)} X_{A_{p}(v)}(u(p))
$$

for $v \in T_{p} P$. In general, $\nabla_{A}$ preserves neither the inner product nor the complex structure on $u^{*} T M / \mathrm{G}$. More precisely, let $J_{u} \in \Omega^{0}\left(P, \operatorname{End}\left(u^{*} T M\right)\right)$ be given by $J_{u}(p):=J_{\pi(p)}(u(p)) \in \operatorname{End}\left(T_{u(p)} M\right)$. This section is equivariant and hence descend to a complex structure, also denoted by $J_{u}$, of the bundle $\operatorname{End}\left(u^{*} T M / \mathrm{G}\right) \rightarrow \Sigma$.

Lemma B.1. The covariant derivative of $J_{u}$ is given by

$$
\nabla_{A} J_{u}=\nabla_{d_{A} u} J(u)+\dot{J}(u),
$$

where $\dot{J}(u) \in \Omega^{1}\left(\Sigma, \operatorname{End}\left(u^{*} T M / \mathrm{G}\right)\right)$ is defined by

$$
\dot{J}(u)_{p}(v):=\left.\frac{\mathrm{d}}{\mathrm{d} t}\right|_{t=0} J_{\gamma(t)}(u(p))
$$

for $v \in T_{p} P$ and a smooth path $\gamma: \mathbb{R} \rightarrow P$ such that $\gamma(0)=p$ and $\dot{\gamma}(0)=v$. Proof. Since $J$ is G-invariant, we have $\mathcal{L}_{X_{\eta}} J=0$ for $\eta \in \mathfrak{g}$ or, equivalently,

$$
\left(\nabla_{X_{\eta}} J\right) \xi=\nabla_{J \xi} X_{\eta}-J \nabla_{\xi} X_{\eta}
$$

Using this formula we obtain

$$
\begin{aligned}
\left(\nabla_{A} J_{u}\right) \xi & =\nabla_{A}\left(J_{u} \xi\right)-J_{u}\left(\nabla_{A} \xi\right) \\
& =\nabla\left(J_{u} \xi\right)-J_{u} \nabla \xi+\nabla_{J_{u} \xi} X_{A}(u)-J_{u} \nabla_{\xi} X_{A}(u) \\
& =\left(\nabla_{d u} J(u)+\dot{J}(u)\right) \xi+\left(\nabla_{X_{A}(u)} J(u)\right) \xi \\
& =\left(\nabla_{d_{A} u} J(u)+\dot{J}(u)\right) \xi
\end{aligned}
$$

as claimed.

It follows from Lemma B.1 that the complex linear part of the connection $\nabla_{A}$ is the connection $\tilde{\nabla}_{A}$ on $u^{*} T M / \mathrm{G}$ given by

$$
\begin{aligned}
\tilde{\nabla}_{A} \xi & :=\nabla_{A} \xi-\frac{1}{2} J_{u}\left(\nabla_{A} J_{u}\right) \xi \\
& =\nabla \xi+\nabla_{\xi} X_{A}(u)-\frac{1}{2} J_{u}\left(\nabla_{d_{A} u} J(u)+\dot{J}(u)\right) \xi
\end{aligned}
$$

Lemma B.2. $\tilde{\nabla}_{A}$ is a Hermitian connection on $u^{*} T M / \mathrm{G}$. 
Proof. We shall use the identity $\left\langle\left(\nabla_{\xi_{1}} J\right) \xi_{2}, \xi_{3}\right\rangle+$ cyclic $=0$. By equation (B.1), we have

$$
\begin{aligned}
& \left\langle\xi_{1}, \nabla_{\xi_{2}} X_{\eta}\right\rangle+\left\langle\nabla_{\xi_{1}} X_{\eta}, \xi_{2}\right\rangle \\
& =\left\langle J \xi_{1}, J \nabla_{\xi_{2}} X_{\eta}\right\rangle+\left\langle J \nabla_{\xi_{1}} X_{\eta}, J \xi_{2}\right\rangle \\
& =\left\langle J \xi_{1}, \nabla_{\xi_{2}}\left(J X_{\eta}\right)-\left(\nabla_{\xi_{2}} J\right) X_{\eta}\right\rangle+\left\langle\nabla_{J \xi_{1}} X_{\eta}-\left(\nabla_{X_{\eta}} J\right) \xi_{1}, J \xi_{2}\right\rangle \\
& =\left\langle J \xi_{1}, \nabla_{\xi_{2}}\left(J X_{\eta}\right)\right\rangle+\left\langle\nabla_{J \xi_{1}} X_{\eta}, J \xi_{2}\right\rangle-\left\langle J \xi_{1},\left(\nabla_{\xi_{2}} J\right) X_{\eta}\right\rangle-\left\langle\xi_{2},\left(\nabla_{X_{\eta}} J\right) J \xi_{1}\right\rangle \\
& =\left\langle J \xi_{1}, \nabla_{\xi_{2}}\left(J X_{\eta}\right)\right\rangle+\left\langle\nabla_{J \xi_{1}} X_{\eta}, J \xi_{2}\right\rangle+\left\langle X_{\eta},\left(\nabla_{J \xi_{1}} J\right) \xi_{2}\right\rangle \\
& =\left\langle\nabla_{J \xi_{1}}\left(J X_{\eta}\right), \xi_{2}\right\rangle-\left\langle J\left(\nabla_{J \xi_{1}} X_{\eta}\right), \xi_{2}\right\rangle-\left\langle\left(\nabla_{J \xi_{1}} J\right) X_{\eta}, \xi_{2}\right\rangle \\
& =0
\end{aligned}
$$

for $\xi_{1}, \xi_{2} \in T_{x} M$ and $\eta \in \mathfrak{g}$. Here the penultimate equality follows from the fact that $J X_{\eta}$ is a gradient vector field and that $\nabla_{J \xi_{1}} J$ is skew-adjoint. This shows that $\nabla X_{A}(u)$ is a 1-form on $\Sigma$ with values in the bundle of skewHermitian endomorphisms of $u^{*} T M / \mathrm{G}$, and so is $J\left(\nabla_{d_{A} u} J\right)$. Moreover, since

$$
d\left\langle\xi_{1}, \xi_{2}\right\rangle=\left\langle\nabla \xi_{1}, \xi_{2}\right\rangle+\left\langle\xi_{1}, \nabla \xi_{2}\right\rangle-\left\langle\xi_{1}, J \dot{J} \xi_{2}\right\rangle,
$$

the operator $\xi \mapsto \nabla \xi-\frac{1}{2} J \dot{J} \xi$ is a Riemannian connection. Hence, by equation (B.2), $\tilde{\nabla}_{A}$ is a Riemannian connection. By definition, it preserves the complex structure.

Lemma B.3. For every gauge transformation $g \in \mathcal{G}(P)$ and every section $\xi \in \Omega^{0}\left(\Sigma, u^{*} T M / \mathrm{G}\right)$ we have

$$
\nabla_{g^{*} A}\left(g^{-1} \xi\right)=g^{-1} \nabla_{A} \xi, \quad \tilde{\nabla}_{g^{*} A}\left(g^{-1} \xi\right)=g^{-1} \tilde{\nabla}_{A} \xi .
$$

Proof. Since the metric $\langle\cdot, \cdot\rangle_{z}$ is G-invariant for every $z \in \Sigma$, we have

$$
\nabla\left(g^{-1} \xi\right)=g^{-1} \nabla \xi-\nabla_{g^{-1}} X_{g^{-1} d g}\left(g^{-1} u\right) .
$$

Hence the first identity follows from the fact that $g^{*} A=g^{-1} d g+g^{-1} A g$ and that $\nabla_{g^{-1} \xi} X_{g^{-1} \eta g}\left(g^{-1} x\right)=g^{-1} \nabla_{\xi} X_{\eta}(x)$. The second identity follows from the first and the fact that $J_{z}$ is G-invariant for every $z$.

Lemma B.4. Suppose that $J$ is independent of $z \in \Sigma$. Then the curvature of the connection $\nabla_{A}$ is the equivariant and horizontal 2-form $F^{\nabla_{A}} \in$ $\Omega^{2}\left(P, \operatorname{End}\left(u^{*} T M\right)\right)$ given by

$$
F^{\nabla_{A}}\left(v_{1}, v_{2}\right) \xi=R\left(d_{A} u(p) v_{1}, d_{A} u(p) v_{2}\right) \xi+\nabla_{\xi} X_{F_{A}\left(v_{1}, v_{2}\right)}(u(p))
$$

for $v_{1}, v_{2} \in T_{p} P$ and $\xi \in T_{u(p)} M$, where $R \in \Omega^{2}(M$, $\operatorname{End}(T M))$ is the Riemann curvature tensor of the metric $\langle\cdot, \cdot\rangle=\omega(\cdot, J \cdot)$. This 2-form descends to a 2-form on $\Sigma$ with values in $\operatorname{End}\left(u^{*} T M / \mathrm{G}\right)$, also denoted by $F^{\nabla_{A}}$.

Proof. Given a map $\mathbb{R}^{2} \rightarrow M:(s, t) \mapsto u(s, t)$, a vector field $\xi(s, t) \in$ $T_{u(s, t)} M$ along $u$, and a G-connection $A=\Phi d s+\Psi d t$, where $\Phi, \Psi: \mathbb{R}^{2} \rightarrow \mathfrak{g}$, 
we denote

$$
\begin{aligned}
v_{s}: & :=\partial_{s} u+X_{\Phi}(u), \quad v_{t}:=\partial_{t} u+X_{\Psi}(u), \\
\nabla_{A, s} \xi & :=\nabla_{s} \xi+\nabla_{\xi} X_{\Phi}(u), \quad \nabla_{A, t} \xi:=\nabla_{t} \xi+\nabla_{\xi} X_{\Psi}(u) .
\end{aligned}
$$

Then the assertion can be restated in the form

$$
\nabla_{A, s} \nabla_{A, t} \xi-\nabla_{A, t} \nabla_{A, s} \xi=R\left(v_{s}, v_{t}\right) \xi+\nabla_{\xi} X_{\partial_{s} \Psi-\partial_{t} \Phi+[\Phi, \Psi]}(u) .
$$

To prove this, we use the identities

$$
\begin{aligned}
\nabla_{A, s} \nabla_{A, t} \xi-\nabla_{A, t} \nabla_{A, s} \xi & =\nabla_{s} \nabla_{t} \xi-\nabla_{t} \nabla_{s} \xi+\nabla_{s} \nabla_{\xi} X_{\Psi}(u)-\nabla_{\nabla_{s} \xi} X_{\Psi}(u) \\
& -\nabla_{t} \nabla_{\xi} X_{\Phi}(u)+\nabla_{\nabla_{t} \xi} X_{\Phi}(u) \\
& +\nabla_{\nabla_{\xi}} X_{\Psi} X_{\Phi}(u)-\nabla_{\nabla_{\xi}} X_{\Phi} X_{\Psi}(u), \\
R\left(\partial_{s} u, \partial_{t} u\right) \xi & =\nabla_{s} \nabla_{t} \xi-\nabla_{t} \nabla_{s} \xi \\
R\left(\partial_{s} u, X_{\Psi}(u)\right) \xi & =\nabla_{s} \nabla_{\xi} X_{\Psi}(u)-\nabla_{\nabla_{s} \xi} X_{\Psi}(u)-\nabla_{\xi} X_{\partial_{s} \Psi}(u), \\
R\left(X_{\Phi}(u), X_{\Psi}(u)\right) \xi & =-\nabla_{\xi} X_{[\Phi, \Psi]}(u)+\nabla_{\nabla_{\xi}} X_{\Psi} X_{\Phi}(u)-\nabla_{\nabla_{\xi}} X_{\Phi} X_{\Psi}(u) .
\end{aligned}
$$

The first and second identities are the definition of the connection $\nabla_{A}$ and the curvature tensor $R$. The other identities use the equations

$$
\nabla_{X_{\eta}} Z-\nabla_{Z} X_{\eta}=\left[Z, X_{\eta}\right]=0, \quad \nabla_{Z}\left[X_{\eta_{1}}, X_{\eta_{2}}\right]=\left[\nabla_{Z} X_{\eta_{1}}, X_{\eta_{2}}\right],
$$

for every G-invariant vector field $Z \in \operatorname{Vect}_{\mathrm{G}}(M)$ and all $\eta, \eta_{1}, \eta_{2} \in \mathfrak{g}$.

Now consider the Cauchy-Riemann operator

$$
D:=D_{(u, A)}: \Omega^{0}\left(\Sigma, u^{*} T M / \mathrm{G}\right) \rightarrow \Omega^{0,1}\left(\Sigma, u^{*} T M / \mathrm{G}\right)
$$

given by

$$
D \xi:=\left(\nabla_{A} \xi\right)^{0,1}-\frac{1}{2} J\left(\nabla_{\xi} J\right) \partial_{J, A}(u)
$$

In the case $\bar{\partial}_{J, A}(u)=0$, this operator is the vertical differential of the section $u \mapsto \bar{\partial}_{J, A}(u)$ of the infinite-dimensional vector bundle over the space $C_{\mathrm{G}}^{\infty}(P, M)$ with fibre $\Omega^{0,1}\left(\Sigma, u^{*} T M / \mathrm{G}\right)$ over $u$.

In the following, we denote the Nijenhuis tensor of $J$ by $N \in \Omega^{2}(T M, T M)$. It is given by

$$
\begin{aligned}
N\left(\xi_{1}, \xi_{2}\right) & =\left[\xi_{1}, \xi_{2}\right]+J\left[J \xi_{1}, \xi_{2}\right]+J\left[\xi_{1}, J \xi_{2}\right]-\left[J \xi_{1}, J \xi_{2}\right] \\
& =2 J\left(\nabla_{\xi_{2}} J\right) \xi_{1}-2 J\left(\nabla_{\xi_{1}} J\right) \xi_{2} .
\end{aligned}
$$

Lemma B.5. The complex linear part of $D$ is the operator $\xi \mapsto\left(\tilde{\nabla}_{A} \xi\right)^{0,1}$. Moreover,

$$
D \xi=\left(\tilde{\nabla}_{A} \xi\right)^{0,1}+\frac{1}{4} N\left(\xi, \partial_{J, A}(u)\right)+\frac{1}{2}(J \dot{J} \xi)^{0,1}
$$


Proof. By definition of $\tilde{\nabla}_{A}$, we have

$$
D \xi=\left(\tilde{\nabla}_{A} \xi\right)^{0,1}+\frac{1}{2} J\left(\nabla_{\partial_{J, A}(u)} J(u)+\dot{J}(u)^{0,1}\right) \xi-\frac{1}{2} J\left(\nabla_{\xi} J\right) \partial_{J, A}(u) .
$$

Hence the formula for $D \xi$ follows from the relation between the Nijenhuis tensor and $\nabla J$. Now this equation shows that the operator $\xi \mapsto D \xi-$ $\left(\tilde{\nabla}_{A} \xi\right)^{0,1}$ is complex anti-linear.

\section{Appendix C. Invariant metrics}

Let $M$ be a (complete) Riemannian $m$-manifold. For $v \in T_{x} M$ and $i, j \in$ $\{1,2\}$ there exist linear maps

$$
E_{i}(x, v): T_{x} M \rightarrow T_{\exp _{x}(v)} M, \quad E_{i j}(x, v): T_{x} M \oplus T_{x} M \rightarrow T_{\exp _{x}(v)} M
$$

characterized by the following conditions. If $x: \mathbb{R} \rightarrow M$ is a smooth curve and $v, w: \mathbb{R} \rightarrow x^{*} T M$ are vector fields along $x$, then

$$
\begin{aligned}
\frac{d}{d t} \exp _{x}(v) & =E_{1}(x, v) \dot{x}+E_{2}(x, v) \nabla_{t} v \\
\nabla_{t}\left(E_{1}(x, v) w\right) & =E_{11}(x, v)(w, \dot{x})+E_{12}(x, v)\left(w, \nabla_{t} v\right)+E_{1}(x, v) \nabla_{t} w, \\
\nabla_{t}\left(E_{2}(x, v) w\right) & =E_{21}(x, v)(w, \dot{x})+E_{22}(x, v)\left(w, \nabla_{t} v\right)+E_{2}(x, v) \nabla_{t} w .
\end{aligned}
$$

Here all the expressions are understood pointwise for every $t \in \mathbb{R}$. Note that the map $E_{11}(x, v)\left(w, w^{\prime}\right)$ is not symmetric in $w$ and $w^{\prime}$. It satisfies

$$
E_{11}(x, v)\left(w, w^{\prime}\right)-E_{11}(x, v)\left(w^{\prime}, w\right)=E_{2}(x, v) R\left(w, w^{\prime}\right) v
$$

where $R \in \Omega^{2}(M, \operatorname{End}(T M))$ denotes the curvature tensor. However,

$$
E_{12}(x, v)\left(w, w^{\prime}\right)=E_{21}(x, v)\left(w^{\prime}, w\right),
$$

and $E_{22}(x, v)\left(w, w^{\prime}\right)$ is symmetric in $w$ and $w^{\prime}$ (see [7] for more details). Now let $\mathrm{G} \times M \rightarrow M:(g, x) \mapsto g x$ be a smooth action of a compact Lie group $\mathrm{G}$ with infinitesimal action $\mathfrak{g} \rightarrow \operatorname{Vect}(M): \eta \mapsto X_{\eta}$. Assume that $M$ is equipped with a G-invariant Riemannian metric.

Lemma C.1.

$$
\begin{aligned}
X_{\eta}\left(\exp _{x}(v)\right)= & E_{1}(x, v) X_{\eta}(x)+E_{2}(x, v) \nabla_{v} X_{\eta}(x), \\
\nabla_{E_{i}(x, v) w} X_{\eta}\left(\exp _{x}(v)\right)= & E_{i 1}(x, v)\left(w, X_{\eta}(x)\right)+E_{i 2}(x, v)\left(w, \nabla_{v} X_{\eta}(x)\right) \\
& +E_{i}(x, v) \nabla_{w} X_{\eta}(x) .
\end{aligned}
$$

Proof. Since the group action preserves geodesics, $g \exp _{x}(v)=\exp _{g x}(g v)$. Differentiate this identity with respect to $g$ to obtain the first identity. To prove the second differentiate, the first identity covariantly and use the definition of $E_{i}$ and $E_{i j}$. For more details, see [7]. 
For each $x \in M$ denote by $L_{x}: \mathfrak{g} \rightarrow T_{x} M$ the infinitesimal action, i.e. $L_{x} \eta=X_{\eta}(x)$. Given a map $u: \mathbb{R}^{2} \rightarrow M$, a vector field $\xi: \mathbb{R}^{2} \rightarrow u^{*} T M$ along $u$, a function $\eta: \mathbb{R}^{2} \rightarrow \mathfrak{g}$, and a G-connection $A=\Phi d s+\Psi d t$, where $\Phi, \Psi: \mathbb{R}^{2} \rightarrow \mathfrak{g}$, we denote

$$
\begin{aligned}
& v_{s}=\partial_{s} u+X_{\Phi}(u), \quad v_{t} \quad=\partial_{t} u+X_{\Psi}(u), \\
& \nabla_{A, s} \xi=\nabla_{s} \xi+\nabla_{\xi} X_{\Phi}(u), \quad \nabla_{A, t} \xi=\nabla_{t} \xi+\nabla_{\xi} X_{\Psi}(u), \\
& \nabla_{A, s} \eta=\partial_{s} \eta+[\Phi, \eta], \quad \nabla_{A, t} \eta=\partial_{t} \eta+[\Psi, \eta] .
\end{aligned}
$$

Define $\rho \in \Omega^{2}(M, \mathrm{G})$ by

$$
\left\langle\eta, \rho\left(\xi, \xi^{\prime}\right)\right\rangle:=\left\langle\nabla_{\xi} X_{\eta}(x), \xi^{\prime}\right\rangle=-\left\langle\nabla_{\xi^{\prime}} X_{\eta}(x), \xi\right\rangle
$$

for $\xi, \xi^{\prime} \in T_{x} M$ and $\eta \in \mathfrak{g}$.

Lemma C.2. With the above notation, we have

$$
\begin{aligned}
& \nabla_{A, s} L_{u} \eta-L_{u} \nabla_{A, s} \eta=\nabla_{v_{s}} X_{\eta}(u), \quad \nabla_{A, t} L_{u} \eta-L_{u} \nabla_{A, t} \eta=\nabla_{v_{t}} X_{\eta}(u), \\
& \nabla_{A, s} L_{u}^{*} \xi-L_{u}^{*} \nabla_{A, s} \xi=\rho\left(v_{s}, \xi\right), \quad \nabla_{A, t} L_{u}^{*} \xi-L_{u}^{*} \nabla_{A, t} \xi=\rho\left(v_{t}, \xi\right)
\end{aligned}
$$

Proof. See [7].

Now let $M^{*}$ denote the subset of all points $x \in M$ with finite isotropy subgroup $\mathrm{G}_{x}:=\{g \in \mathrm{G} \mid g x=x\}$. Thus $x \in M^{*}$ if and only if the linear map $L_{x}: \mathfrak{g} \rightarrow T_{x} M$ is injective. Hence, for every map $u_{0}: \mathbb{R}^{2} \rightarrow M^{*}$ there exists a unique G-connection $A_{0}=\Phi_{0} d s+\Psi_{0} d t$ such that

$$
L_{u_{0}}^{*} v_{0 s}=L_{u_{0}}^{*} v_{0 t}=0,
$$

where

$$
v_{0 s}:=\partial_{s} u_{0}+L_{u_{0}} \Phi_{0}, \quad v_{0 t}:=\partial_{t} u_{0}+L_{u_{0}} \Psi_{0} .
$$

Let $\xi_{0}: \mathbb{R}^{2} \rightarrow u_{0}^{*} T M$ be a vector field along $u_{0}$, consider the map

$$
u(s, t):=\exp _{u_{0}(s, t)}\left(\xi_{0}(s, t)\right),
$$

and abbreviate

$$
\varphi_{0}:=\Phi-\Phi_{0}, \quad \psi_{0}:=\Psi-\Psi_{0} .
$$

\section{Lemma C.3.}

$$
\begin{aligned}
& L_{u} \varphi_{0}=v_{s}-E_{1}\left(u_{0}, \xi_{0}\right) v_{0 s}-E_{2}\left(u_{0}, \xi_{0}\right) \nabla_{A_{0}, s} \xi_{0}, \\
& L_{u} \psi_{0}=v_{t}-E_{1}\left(u_{0}, \xi_{0}\right) v_{0 t}-E_{2}\left(u_{0}, \xi_{0}\right) \nabla_{A_{0}, t} \xi_{0} .
\end{aligned}
$$

Proof. We compute

$$
\begin{aligned}
L_{u}\left(\Phi-\Phi_{0}\right)= & X_{\Phi}(u)-E_{1}\left(u_{0}, \xi_{0}\right) X_{\Phi_{0}}\left(u_{0}\right)-E_{2}\left(u_{0}, \xi_{0}\right) \nabla_{\xi_{0}} X_{\Phi_{0}}\left(u_{0}\right) \\
= & \partial_{s} u-E_{1}\left(u_{0}, \xi_{0}\right) \partial_{s} u_{0}-E_{2}\left(u_{0}, \xi_{0}\right) \nabla_{s} \xi_{0} \\
& +X_{\Phi}(u)-E_{1}\left(u_{0}, \xi_{0}\right) X_{\Phi_{0}}\left(u_{0}\right)-E_{2}\left(u_{0}, \xi_{0}\right) \nabla_{\xi_{0}} X_{\Phi_{0}}\left(u_{0}\right) \\
= & v_{s}-E_{1}\left(u_{0}, \xi_{0}\right) v_{0 s}-E_{2}\left(u_{0}, \xi_{0}\right) \nabla_{A_{0}, s} \xi_{0} .
\end{aligned}
$$

Here the first equation follows from Lemma C.1, the second from the definition of $E_{i}$, and the last from the definitions of $v_{s}$ and $v_{0 s}$. 
In the proof, we did not use the fact that $L_{u_{0}}^{*} v_{0 s}=L_{u_{0}}^{*} v_{0 t}=0$. Now suppose $L_{u_{0}}^{*} \xi_{0}=0$. Then, by Lemma C.2,

$$
L_{u_{0}}^{*} \nabla_{A_{0}, s} \xi_{0}=-\rho\left(v_{0 s}, \xi_{0}\right), \quad L_{u_{0}}^{*} \nabla_{A_{0}, t} \xi_{0}=-\rho\left(v_{0 t}, \xi_{0}\right) .
$$

Abbreviating $E_{1}=E_{1}\left(u_{0}, \xi_{0}\right)$ and $E_{2}=E_{2}\left(u_{0}, \xi_{0}\right)$, we obtain the following.

Corollary C.4. If $L_{u_{0}}^{*} \xi_{0}=0$, then

$$
\begin{aligned}
& L_{u_{0}}^{*} E_{2}{ }^{-1} L_{u}\left(\Phi-\Phi_{0}\right)=L_{u_{0}}^{*} E_{2}{ }^{-1}\left(v_{s}-E_{1} v_{0 s}\right)+\rho\left(v_{0 s}, \xi_{0}\right), \\
& L_{u_{0}}^{*} E_{2}{ }^{-1} L_{u}\left(\Psi-\Psi_{0}\right)=L_{u_{0}}^{*} E_{2}{ }^{-1}\left(v_{t}-E_{1} v_{0 t}\right)+\rho\left(v_{0 t}, \xi_{0}\right) .
\end{aligned}
$$

Proof. Lemma C.3.

Lemma C.5. Assume $L_{u_{0}}^{*} \xi_{0}=0$ and abbreviate $E_{i}:=E_{i}\left(u_{0}, \xi_{0}\right)$ and $E_{i j}:=$ $E_{i j}\left(u_{0}, \xi_{0}\right)$. Then

$$
\begin{aligned}
L_{u} \nabla_{A, t} \varphi_{0}= & \nabla_{A, t} v_{s}+\nabla_{X_{\varphi_{0}}} X_{\psi_{0}}(u)-\nabla_{v_{t}} X_{\varphi_{0}}(u)-\nabla_{v_{s}} X_{\psi_{0}}(u) \\
- & E_{11}\left(v_{0 s}, v_{0 t}\right)-E_{12}\left(v_{0 s}, \nabla_{A_{0}, t} \xi_{0}\right) \\
- & E_{21}\left(\nabla_{A_{0}, s} \xi_{0}, v_{0 t}\right)-E_{22}\left(\nabla_{A_{0}, s} \xi_{0}, \nabla_{A_{0}, t} \xi_{0}\right) \\
- & E_{1} \nabla_{A_{0}, t} v_{0 s}-E_{2} \nabla_{A_{0}, t} \nabla_{A_{0}, s} \xi_{0} \\
L_{u} \nabla_{A, s} \varphi_{0}= & \nabla_{A, s} v_{s}+\nabla_{X_{\varphi_{0}}} X_{\varphi_{0}}(u)-2 \nabla_{v_{s}} X_{\varphi_{0}}(u) \\
& -E_{11}\left(v_{0 s}, v_{0 s}\right)-E_{12}\left(v_{0 s}, \nabla_{A_{0}, s} \xi_{0}\right) \\
& -E_{21}\left(\nabla_{A_{0}, s} \xi_{0}, v_{0 s}\right)-E_{22}\left(\nabla_{A_{0}, s} \xi_{0}, \nabla_{A_{0}, s} \xi_{0}\right) \\
& -E_{1} \nabla_{A_{0}, s} v_{0 s}-E_{2} \nabla_{A_{0}, s} \nabla_{A_{0}, s} \xi_{0}
\end{aligned}
$$

Proof. We only prove the first identity. The proof of the second is similar. By Lemmas C.2 and C.3,

$$
\begin{aligned}
L_{u} \nabla_{A, t} \varphi_{0} & =\nabla_{A, t} L_{u} \varphi_{0}-\nabla_{v_{t}} X_{\varphi^{0}}(u) \\
& =\nabla_{A, t} v_{s}-\nabla_{A, t}\left(E_{1} v_{0 s}\right)-\nabla_{A, t}\left(E_{2} \nabla_{A_{0}, s} \xi_{0}\right)-\nabla_{v_{t}} X_{\varphi_{0}}(u) .
\end{aligned}
$$

Hence, by the definition of $E_{i j}$ and Lemma C.1,

$$
\begin{aligned}
\nabla_{A, t}\left(E_{1} v_{0 s}\right)= & \nabla_{t}\left(E_{1} v_{0 s}\right)+\nabla_{E_{1} v_{0 s}} X_{\Psi}(u) \\
= & E_{11}\left(v_{0 s}, \partial_{t} u_{0}\right)+E_{12}\left(v_{0 s}, \nabla_{t} \xi_{0}\right) \\
& +E_{11}\left(v_{0 s}, X_{\Psi_{0}}\left(u_{0}\right)\right)+E_{12}\left(v_{0 s}, \nabla_{\xi_{0}} X_{\Psi_{0}}\left(u_{0}\right)\right) \\
& +E_{1} \nabla_{t} v_{0 s}+\nabla_{E_{1} v_{0 s}} X_{\psi_{0}}(u)+E_{1} \nabla_{v_{0 s}} X_{\Psi_{0}}\left(u_{0}\right) \\
= & \nabla_{E_{1} v_{0 s}} X_{\psi_{0}}(u)+E_{11}\left(v_{0 s}, v_{0 t}\right)+E_{12}\left(v_{0 s}, \nabla_{A_{0}, t} \xi_{0}\right)+E_{1} \nabla_{A_{0}, t} v_{0 s},
\end{aligned}
$$


and

$$
\begin{aligned}
\nabla_{A, t}\left(E_{2} \nabla_{A_{0}, s} \xi_{0}\right)= & \nabla_{t}\left(E_{2} \nabla_{A_{0}, s} \xi_{0}\right)+\nabla_{E_{2} \nabla_{A_{0}, s} \xi_{0}} X_{\Psi}(u) \\
= & E_{21}\left(\nabla_{A_{0}, s} \xi_{0}, \partial_{t} u_{0}\right)+E_{22}\left(\nabla_{A_{0}, s} \xi_{0}, \nabla_{t} \xi_{0}\right)+E_{2} \nabla_{t} \nabla_{A_{0}, s} \xi_{0} \\
& +\nabla_{E_{2} \nabla_{A_{0}, s} \xi_{0}} X_{\psi_{0}}(u)+E_{21}\left(\nabla_{A_{0}, s} \xi_{0}, X_{\Psi_{0}}\left(u_{0}\right)\right) \\
& +E_{22}\left(\nabla_{A_{0}, s} \xi_{0}, \nabla_{\xi_{0}} X_{\Psi_{0}}\left(u_{0}\right)\right)+E_{2} \nabla_{\nabla_{A_{0}, s} \xi_{0}} X_{\Psi_{0}}\left(u_{0}\right) \\
= & \nabla_{E_{2} \nabla_{A_{0}, s} \xi_{0}} X_{\psi_{0}}(u)+E_{21}\left(\nabla_{A_{0}, s} \xi_{0}, v_{0 t}\right) \\
& +E_{22}\left(\nabla_{A_{0}, s} \xi_{0}, \nabla_{A_{0}, t} \xi_{0}\right)+E_{2} \nabla_{A_{0}, t} \nabla_{A_{0}, s} \xi_{0} .
\end{aligned}
$$

Inserting these two identities into the previous formula, we obtain

$$
\begin{aligned}
L_{u} \nabla_{A, t} \varphi_{0}= & \nabla_{A, t} v_{s}-\nabla_{v_{t}} X_{\varphi_{0}}(u) \\
& -\nabla_{E_{1} v_{0 s}} X_{\psi_{0}}(u)-\nabla_{E_{2} \nabla_{A_{0}, s} \xi_{0}} X_{\psi_{0}}(u) \\
& -E_{11}\left(v_{0 s}, v_{0 t}\right)-E_{12}\left(v_{0 s}, \nabla_{A_{0}, t} \xi_{0}\right) \\
& -E_{21}\left(\nabla_{A_{0}, s} \xi_{0}, v_{0 t}\right)-E_{22}\left(\nabla_{A_{0}, s} \xi_{0}, \nabla_{A_{0}, t} \xi_{0}\right) \\
& -E_{1} \nabla_{A_{0}, t} v_{0 s}-E_{2} \nabla_{A_{0}, t} \nabla_{A_{0}, s} \xi_{0} .
\end{aligned}
$$

Now the result follows from Lemma C.3.

\section{References}

[1] M.F. Atiyah and R. Bott, The Yang-Mills equations over Riemann surfaces, Phil. Trans. R. Soc. Lond. A 308 (1982), 523-615.

[2] C. Cieliebak, R. Gaio, I. Mundet i Riera and D.A. Salamon, Invariants of Hamiltonian group actions, J. Symplectic Geom. 1(3) (2002), 543-645.

[3] C. Cieliebak, A.R. Gaio and D.A. Salamon, J-homolorphic curves, moment maps and invariants of Hamiltonian group actions, Int. Math. Res. Notes 10 (2000), 831-882.

[4] S.K. Donaldson and P.B. Kronheimer, The Geometry of Four-Manifolds, Oxford: Clarendon Press (Oxford Mathematical Monographs), 1990.

[5] S. Dostoglou and D.A. Salamon, Cauchy-Riemann operators, self-duality, and the spectral flow, in, 'First European Congress of Mathematics, Volume I, Invited Lectures (Part 1)' (A. Joseph, F. Mignot, F. Murat, B. Prum, R. Rentschler, eds.) Progress in Mathematics, 119, Birkhäuser Verlag (Basel), 1994, pp. 511-545.

[6] S. Dostoglou and D.A. Salamon, Self-dual instantons and holomorphic curves, Ann. Math. 139 (1994), 581-640.

[7] A.R. Gaio, J-holomorphic curves and moment maps, Ph.D. thesis, University of Warwick, 1999.

[8] D. Gilbarg and N.S. Trudinger, Elliptic Partial Differential Equations of the Second Order, Springer Verlag (Berlin), 1983.

[9] M.Gromov, Pseudoholomorphic curves in symplectic manifolds, Invent. Math. 82 (1985), 307-347.

[10] F. Kirwan, Cohomology of Quotients in Symplectic and Algebraic Geometry, Princeton University Press, 1984. 
[11] D. McDuff and D.A. Salamon, J-holomorphic Curves and Symplectic Topology, 52, AMS Colloquium Publications, Providence, RI, 52, 2004.

[12] D. McDuff and D.A. Salamon, Introduction to Symplectic Topology, Oxford University Press, 1995, 2nd ed., 1998.

[13] I. Mundet i Riera, Yang-Mills-Higgs theory for symplectic fibrations, Ph.D. thesis, Madrid, 1999.

[14] I. Mundet i Riera, Hamiltonian Gromov-Witten invariants, preprint, 2000, math.SG/0002121.

[15] Y. Ruan and G. Tian, A mathematical theory of quantum cohomology, J. Diff. Geom. 42 (1995), 259-367.

[16] D.A. Salamon, Quantum products for mapping tori and the Atiyah-Floer conjecture, Am. Math. Soc. Transl. 196 (1999), 199-235. Revised in December 2000, http://www.math.ethz.ch/salamon.

[17] K. Uhlenbeck, Connections with $L^{p}$-bounds on curvature, Commun. Math. Phys. 83 (1982), 31-42.

[18] K. Wehrheim, Uhlenbeck Compactness, EMS Series of Lectures in Mathematics, Germany: European Mathematical Society, 2004.

Faculdade de Ciências da Universidade do Porto,

Rua do Campo Alegre 687,

4169-007 Porto, Portugal.

E-mail address: argaio@fc.up.pt

ETH-ZENTRUM,

RÄMISTRASSE 101,

8092 ZÜRICH, SWITZERLAND

E-mail address: salamon@math.ethz.ch

Received 28/9/2005, accepted ???.

The first author would like to thank FCT and Dep. Mat. Pura, Univ. Porto, for financial support. 
